\title{
Studying Protein Organization in Cellular Membranes by
}

\author{
High-Resolution Microscopy
}

\begin{abstract}
Dissertation
in partial fulfillment of the requirements for the degree "Doctor of Natural Sciences (Dr. rer. nat.)" in the Molecular Biology Program at the Georg August University Göttingen, Faculty of Biology
\end{abstract}

submitted by

Sinem Saka Kırlı

born in

Ankara, Turkey

Göttingen, September 2013 



\section{Members of the Thesis Committee}

Prof. Dr. Silvio Rizzoli (Supervisor and first referee)

Department of Neuro- and Sensory Physiology

Georg-August-University of Göttingen Medical Center

Göttingen, Germany

Prof. Dr. Michael Kessel (Second referee)

Department of Developmental Biology

Max Planck Institute for Biophysical Chemistry

Göttingen, Germany

Prof. Dr. Mikael Simons

Department of Cellular Neuroscience

Max Planck Institute for Experimental Medicine

Göttingen, Germany

\section{Additional Members of the Examination Board}

\section{Prof. Dr. Stefan W. Hell}

Department of NanoBiophotonics

Max Planck Institute for Biophysical Chemistry

Göttingen, Germany

Prof. Dr. Detlef Doenecke

Department of Molecular Biology

Georg-August-University of Göttingen Medical Center

Göttingen, Germany

Prof. Dr. Michael Thumm

Department of Biochemistry II

Georg-August-University of Göttingen

Göttingen, Germany 

I hereby declare that I prepared this dissertation independently and with no other sources and aids than quoted. 

"The law of evolution is that the strongest survives."

"Yes, and the strongest, in the existence of any social species, are those who are most social. In human terms, most ethical."

Ursula Le Guin, The Dispossessed

In memory of the strongest ones: those who sacrificed their lives for people whose faces they have never seen, "despite knowing that the best and the most real thing is to live..." 



AIST OF PUBLICATIONS.

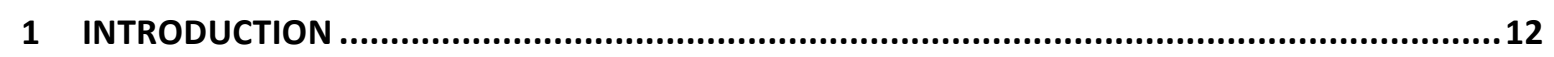

1.1 A Brief History of the Plasma Membrane and Early Models ............................................12

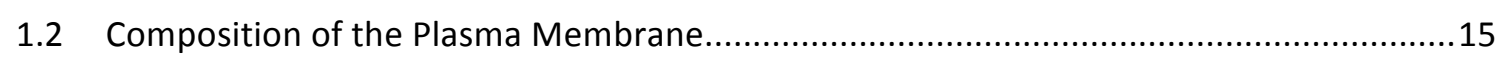

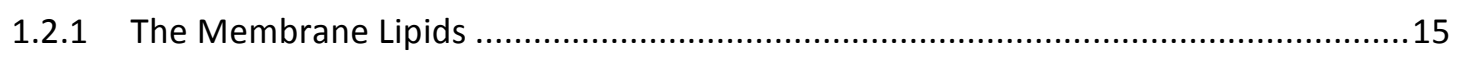

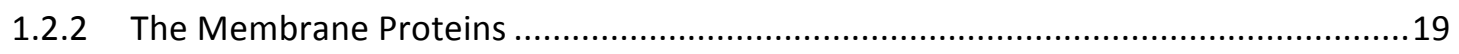

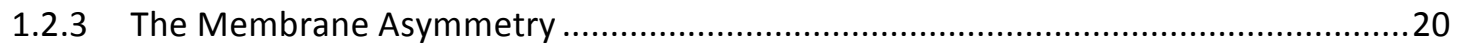

1.3 Modern Models of Membrane Organization: The Lipid Side ...........................................21

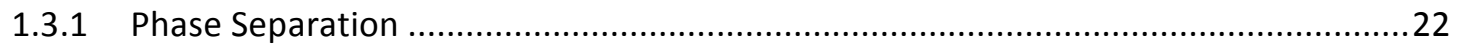

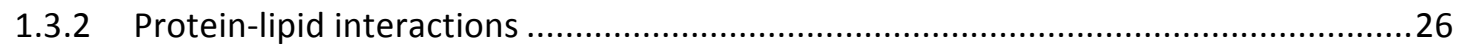

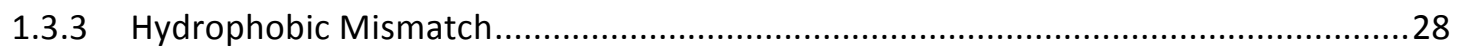

1.4 Modern Models of Membrane Organization: The Protein Side ........................................29

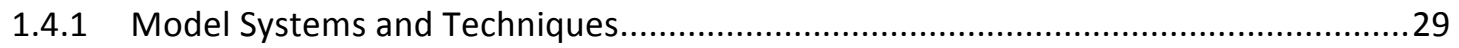

1.4.2 Examples of Specialized Protein Domains in Cellular Membranes .............................33

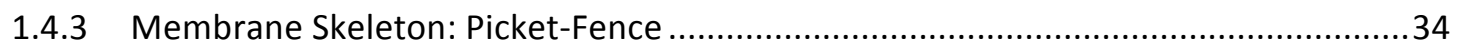

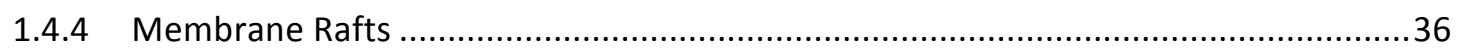

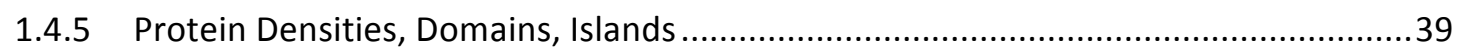

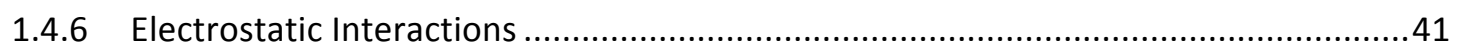

1.5 Technical Perspective: High-Resolution Microscopy........................................................

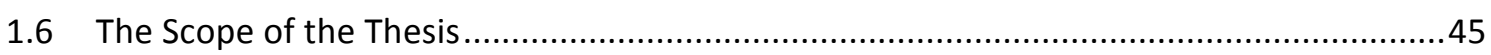

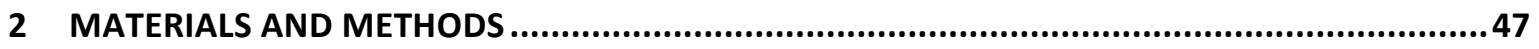

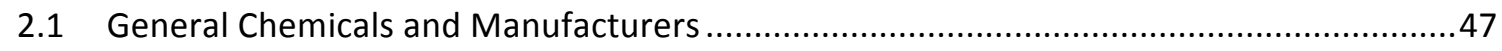

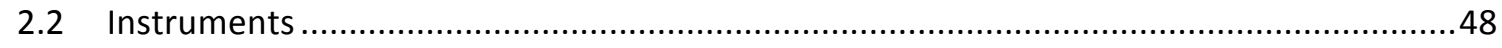

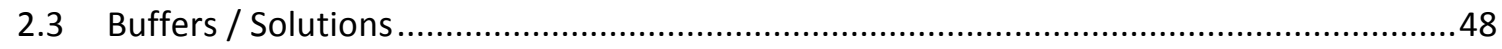

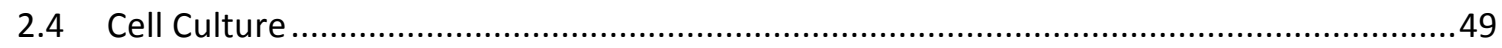




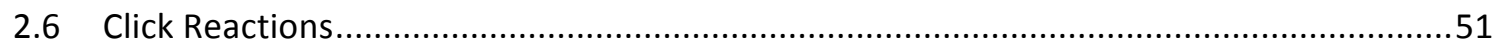

2.6.1 Click Labeling of Protein Modifiers ....................................................................5

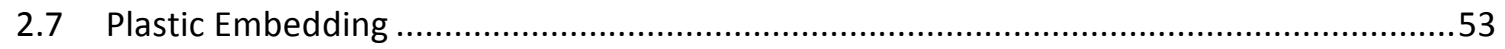

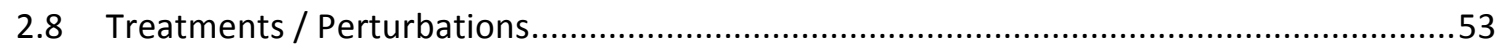

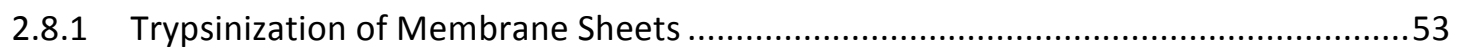

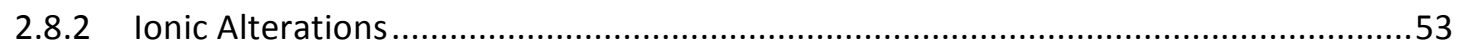

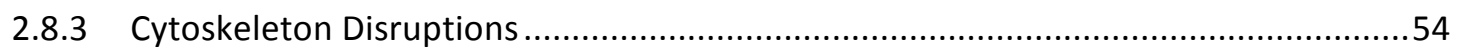

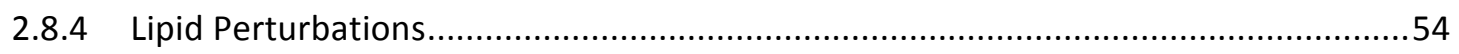

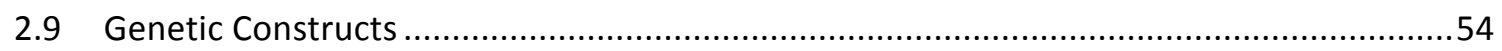

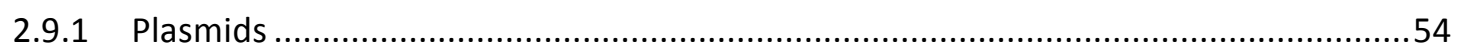

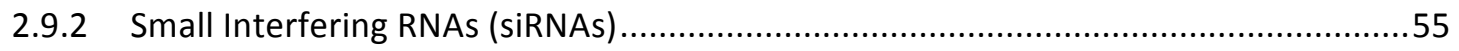

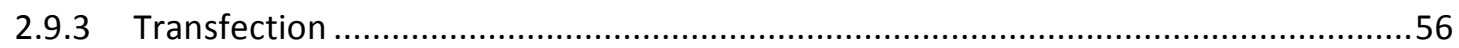

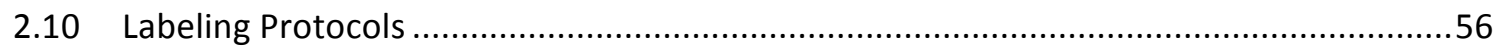

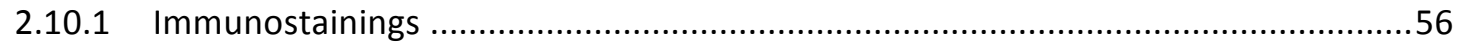

2.10.2 Labeling Whole Cells from Extracellular Side ................................................58

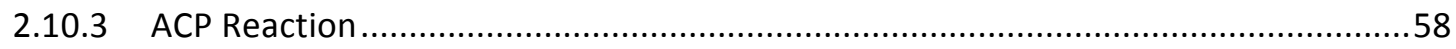

2.10.4 Aptamer Labeling ............................................................................... 58

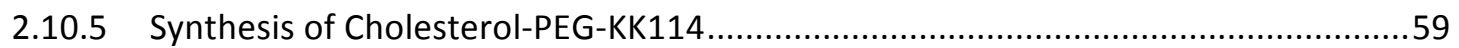

2.10.6 Incorporation of Fluorescent Lipid Analogues into Membrane Sheets ..................60

2.10.7 Transfer of Fluorescent Probes to Plasma Membranes of Living Cells ...................61

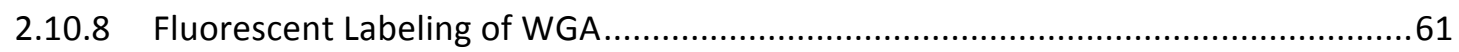

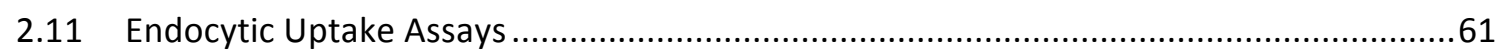

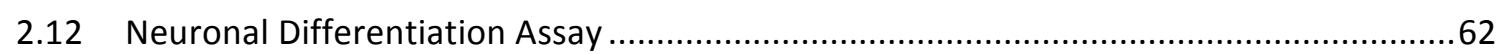

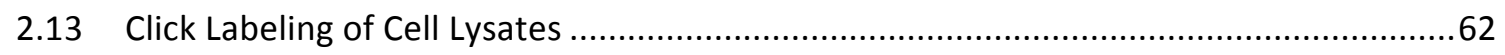

2.14 SDS-Polyacrylamide Gel Electrophoresis (PAGE) ................................................62

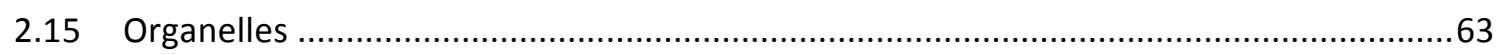

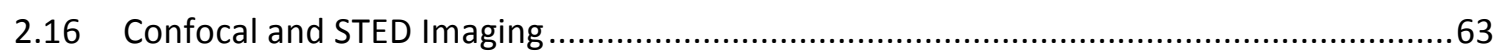

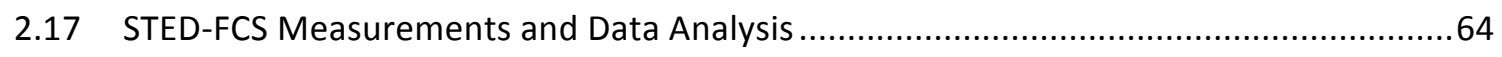

2.18 Sample Preparation and Imaging For SIMS / COIN ..............................................66

2.18.1 Preparation of Hippocampal Neurons for Imaging of Organellar Turnover ............66

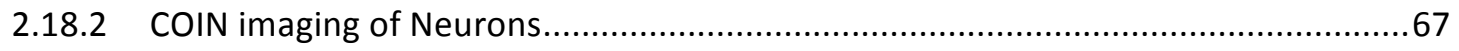

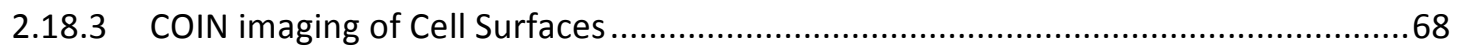

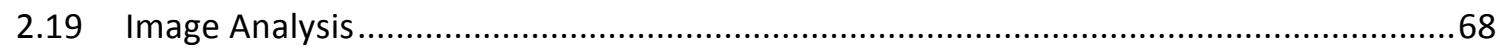

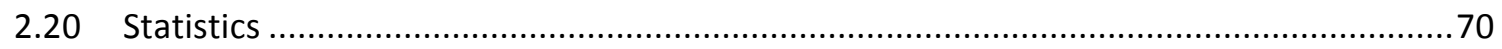




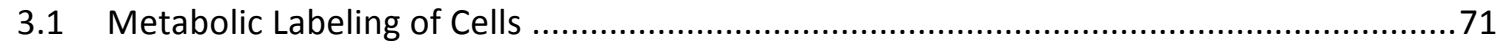

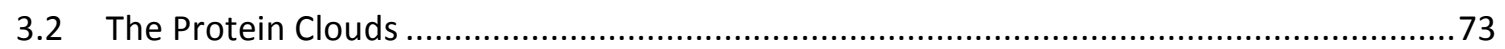

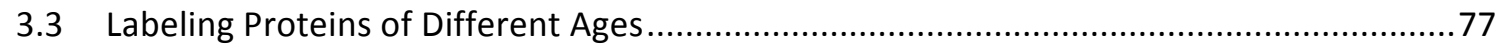

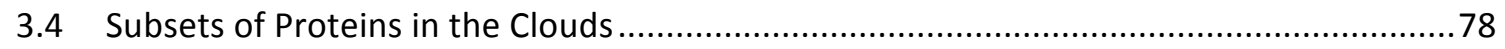

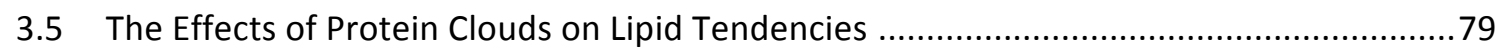

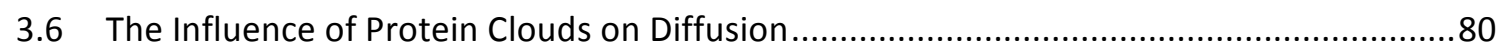

3.7 The Effect of Increasing the Amount of Protein at the Membrane ....................................83

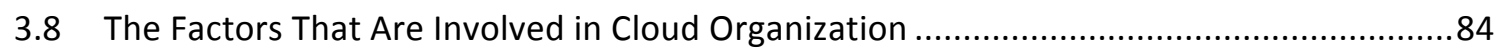

3.8.1 The Stability of the Clouds under Different Conditions ............................................8

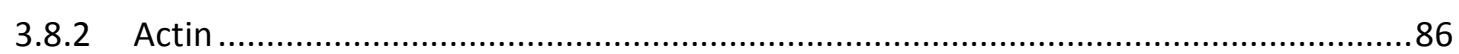

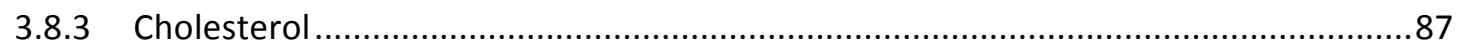

3.9 Distributions of Specific Proteins within the Clouds ...................................................... 91

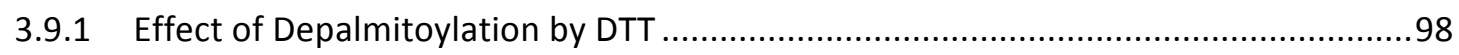

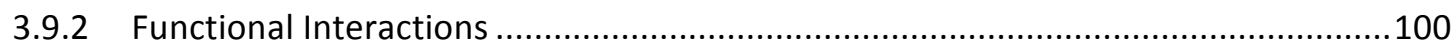

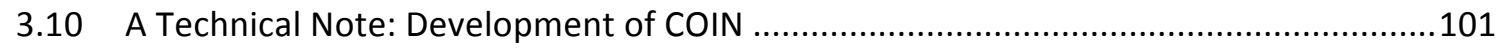

3.10.1 Secondary Isotope Mass Spectrometry …….....................................................101

3.10.2 Correlation of STED Microscopy with Isotopic Imaging ......................................103

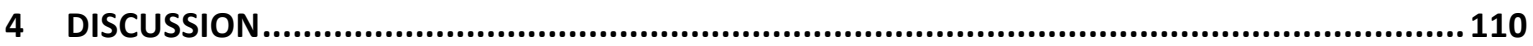

4.1 The General Model: Protein Clouds as a Membrane Organizing Principle ......................110

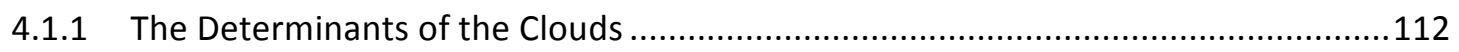

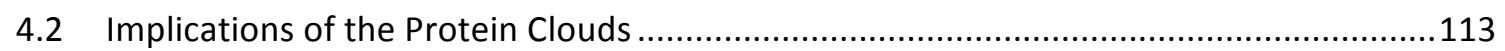

4.2.1 Implications Regarding the Membrane Organization ...........................................113

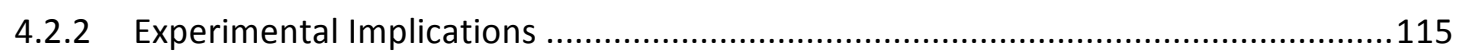

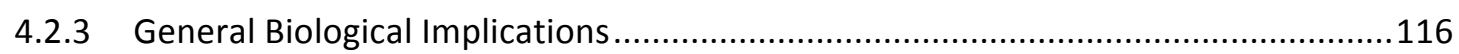

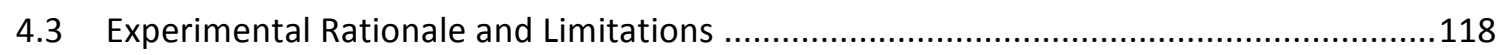

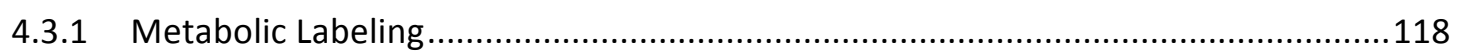

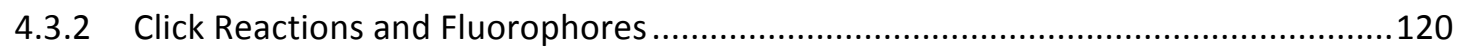

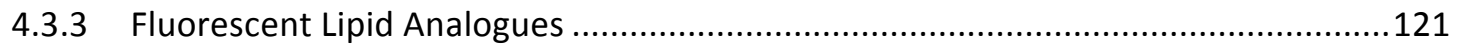

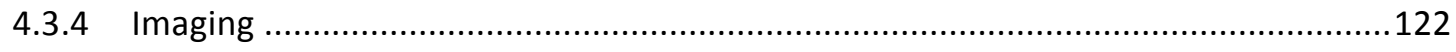

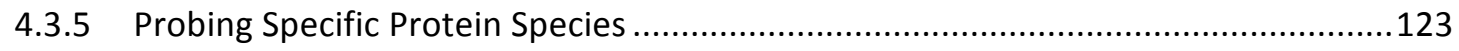

4.3.6 Limitations Related to Membrane Sheets …........................................................124

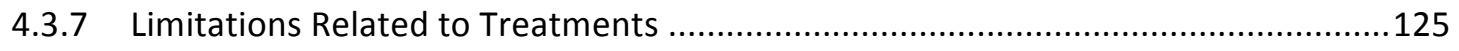

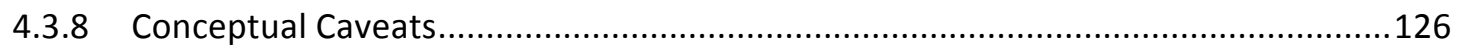


4.4 Compatibility of Protein Clouds with Previous Models .............................................127

4.4.1 Membrane Skeleton and Diffusion Measurements ............................................128

4.4.2 Membrane Rafts ................................................................................. 129

4.4.3 Specific Protein Clusters............................................................................ 131

4.4.4 General Multi-Protein Domains ...................................................................132

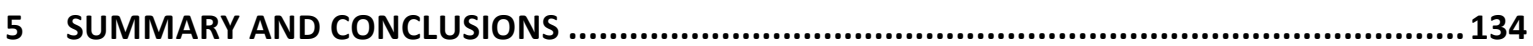

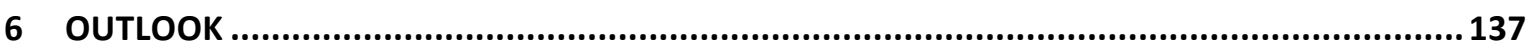

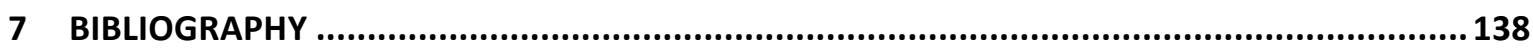




\section{LIST OF PUBLICATIONS}

The results of this dissertation are presented in the following manuscripts submitted for publication:

Saka, S.K., Honigmann, A., Vogts, A., Wessels, J., Eggeling, C., Hell. S.W., Lang, T., Rizzoli, S.O. Multi-protein assemblies underlie the mesoscale organization of the plasma membrane. Submitted.

Saka, S.K., Vogts, A., Kröhnert, K., Hillion, F., Rizzoli, S.O., Wessels, J. Correlated Optical Isotopic Nanoscopy. Submitted.

Saka, S.K. Light Microscopy and Resolution. in Super-Resolution Microscopy Techniques in the Neurosciences (Fornasiero, E. F. \& Rizzoli, S. O.). In press.

Saka, S.K. Super-Resolution Microscopy: Principles, Techniques, and Applications. in SuperResolution Microscopy Techniques in the Neurosciences (Fornasiero, E. F. \& Rizzoli, S. O.). In press.

Saka, S. \& Rizzoli, S.O. Super-resolution imaging prompts re-thinking of cell biology mechanisms: selected cases using stimulated emission depletion microscopy. Bioessays 34 (5), 386-395 (2012). 


\section{ACKNOWLEDGMENTS}

I would like to present my deepest thanks to Silvio Rizzoli for being a real "Doktorvater" and mentor to me in every aspect. Throughout these years, I have felt the privileges of working with him for countless times. Whenever I was worried, he gave me the kick I needed and opened me the way to better myself. I also acknowledge Silvio's immense support for data analysis.

I would like to thank Mikael Simons and Micheal Kessel for being part of my thesis committee and their suggestions for the project. I thank Stefan Hell, Detlef Doenecke and Michael Thumm for joining my extended thesis committee.

I thank Christina Schäfer and Katharina Kröhnert for technical assistance and for the neuronal cultures and COIN embeddings; Ingrid-Cristiana Vreja for help with the membrane sheet preparations and knockdowns, and Natalia Revelo for providing the fixable membrane dye used in SIMS imaging.

I would like to thank Vladimir Belov for the fluorescently labeled lipids and Alf Honigmann, Christian Eggeling and Stefan Hell for the contribution of STED-FCS experiments and inspiring discussions. I thank François Hillion, Johannes Wessels and Angela Vogts for their contributions to the SIMS experiments and the imaging opportunity. I would like to thank Thorsten Lang for his suggestions and the helpful discussions. I appreciate Bianca Rizzoli's time and effort in language editing of our scientific manuscripts. I thank Reinhard Jahn for the kind gift of synaptophysin antibodies and for his scientific input and advices. I thank Kai Simons for his valuable comments and suggestions.

I am vary happy that I had the chance to share not only the lab but also scientific experiences, horizon-broadening lunches and fun with my lab mates Natalia Revelo, Eugenio Fornasiero, Ingrid Vreja, Nora Wender, Sven Truckenbrodt, Nikhil Sasidharan, Annette Denker, Benjamin Wilhelm, Dirk Kamin, Sina Barych, Katharina Kröhnert and Christina Schäfer and with my office mates Felipe Opazo, William Zhang and Peer Hoopmann.

I would like to acknowledge the funding from the University of Göttingen through a Dorothea Schözer PhD Fellowship and later from Boehringer Ingelheim Fonds through a BIF PhD Fellowship. I feel very fortunate to be accepted to the BIF family and I appreciate the warm welcome and care 
from Claudia Walther, Anja Hoffman, and Sandra Schedler. I am also thankful to BIF for the unique opportunity to participate in the $105^{\text {th }}$ International Titisee Conference, which enabled me to meet all the prominent scientists I have cited in this thesis in the flesh. I would also like to acknowledge the travel grants from BIF, GGNB and EMBO that allowed me to attend to wonderful meetings.

I believe that the IMPRS Molecular Biology program was a great opportunity for me and I am thankful to Steffen Burkhardt and Kerstin Grueniger for taking care of many details and difficulties.

I am delighted to have met my dear program friends Carlos Eduardo Lima da Cunha, "Chepe" Florez Weidinger, Melanie Haas, Iris Finci, Helena Magliarelli, Lope Florez Weidinger, Christian Schulz and many more. I am grateful to them for the social circle they embraced me in and making Göttingen (as well as many other cities) so enjoyable.

I am indebted to Selcen Öztürk, Samir Karaca, Kevser Gençalp, Halenur Yavuz, Metin Aksu and the lovely Turkish science community in Göttingen for "resisting" with me in times of trouble and helping me feel warmer in Germany.

I cannot appreciate enough the endless support of my family, even at the times I was supposed to be there for them.

Finally, the best of all was going through everything with Koray Kırl. At the end of the day, even if everything fails miserably, knowing that there is a home I can return is one of the best luxuries in life. He has become my "home". 


\section{ABSTRACT}

Most membrane proteins are found in clusters in the plasma membrane. For abundant proteins it is apparent that the clusters also tend to have patterned distributions, rather than being randomly scattered. To reveal the mechanism behind this observation, I have investigated all proteins in the plasma membrane simultaneously. This was achieved by large-scale metabolic labeling of proteins through incorporation of a non-canonical amino acid analogue and fluorescent tagging by click chemistry. Plasma membrane sheets were obtained from labeled PC12 and COS-7 cells and imaged by super-resolution stimulated emission depletion (STED) microscopy. Membrane proteins were found to form a mosaic like pattern. Heterogeneous domains, highly enriched in proteins, were distributed on a protein-poor background. I have termed these high-abundance domains "protein clouds" and assayed the contribution of different factors to their formation and maintenance. The protein cloud pattern was surprisingly robust and resisted to a variety of manipulations including changes in ionic composition and strength, decrease in protein density, disruption of cytoskeletal elements, and hydrolysis of phospholipids and sphingomyelin. Although actin disruption did not abolish the clouds, it resulted in formation of larger domains. Accordingly, actin was identified as a secondary factor that borders the clouds and prevents their coalescence. Depletion of cholesterol almost completely eliminated the protein clouds, in reversible fashion, suggesting that cholesterol is the major factor for the cloud patterning.

The cloud pattern was confirmed by two additional, independent techniques: first, label-free secondary ion mass spectrometry (SIMS) and second, STED fluorescence correlation spectroscopy (STED-FCS). For reliable application of SIMS, a novel experimental scheme, named correlated optical isotopic nanoscopy (COIN), was developed and verified.

To find out the relevance of clouds to specific proteins, distributions of different classes of proteins were investigated. All of the specific proteins analyzed were enriched in the protein clouds, but displayed differential enrichment profiles. Many proteins were preferentially located in particular areas, such as the edges or centers of the clouds. Some functional partners were observed to show similar profiles. I conclude that enrichment of proteins in the clouds can act as a basic, low-hierarchy principle of membrane patterning, underlying the distributions of specific proteins. 


\section{LIST OF FIGURES}

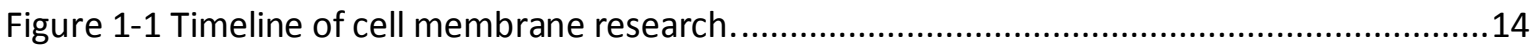

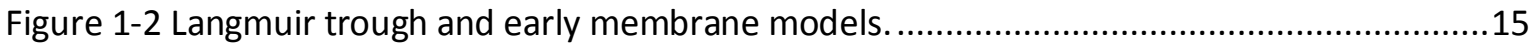

Figure 1-3 Structures of major membrane lipid species.............................................................16

Figure 1-4 Interaction models of cholesterol with other lipids ....................................................25

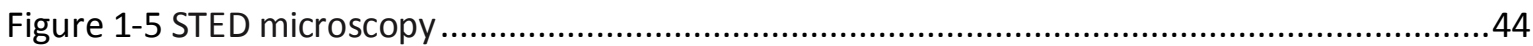

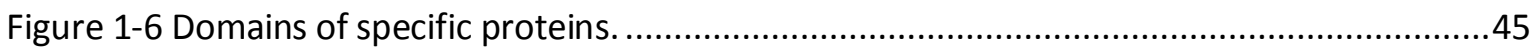

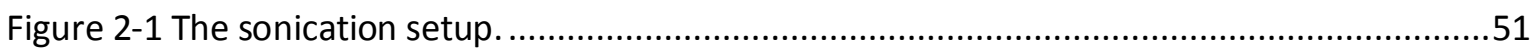

Figure 2-2 Lipid phase partitioning of Chol-PEG-KK114 in GUVs. ...............................................60

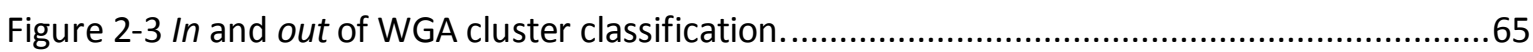

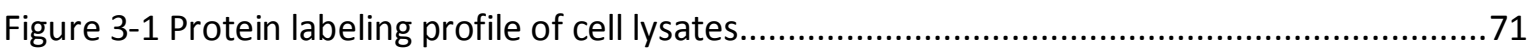

Figure 3-2 Assaying the overall fitness of metabolically labeled PC12 cells. .................................72

Figure 3-3 Proteins in the membrane are organized into protein clouds......................................74

Figure 3-4 Protein clouds under different sample preparation conditions. ....................................75

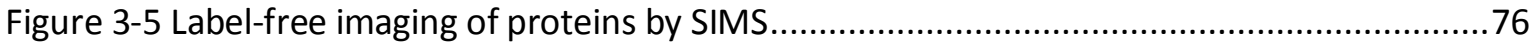

Figure 3-6 Two-color STED imaging of proteins of different ages. ................................................78

Figure 3-7 Different classes of proteins localize to the protein clouds..........................................79

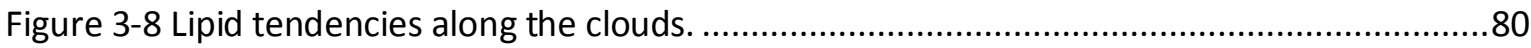

Figure 3-9 Mobilities of the cholesterol analogue and WGA are slower in the protein clouds. ......82

Figure 3-10 Effects of protein overexpression on the general protein organization........................84

Figure 3-11 Protein cloud organization persists after many harsh treatments. ..............................85

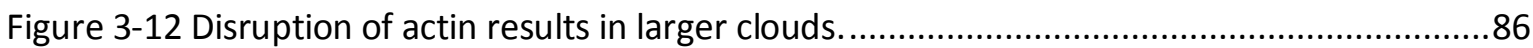

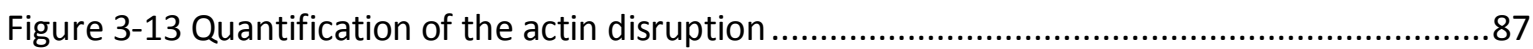

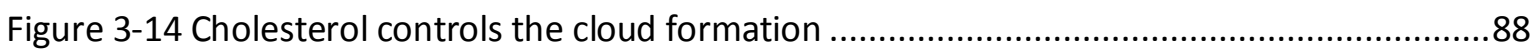

Figure 3-15 Effect of cholesterol on clouds can be reproduced on organellar membranes.............89

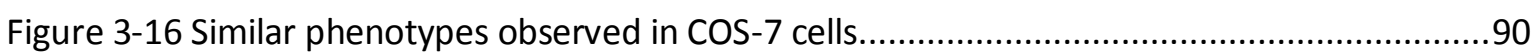

Figure 3-17 STED-FCS measurements on living cells treated with COase......................................91

Figure 3-18 Distributions of several specific proteins in the protein clouds................................95

Figure 3-19 Actin disruption does not change the TfnR localization within clouds ........................96

Figure 3-20 Distributions of specific proteins in COS-7 membranes ..........................................97

Figure 3-21 Distributions of proteins by monovalent probes ........................................................97

Figure 3-22 Analysis of surface staining of proteins on whole PC12 cells. ...................................98

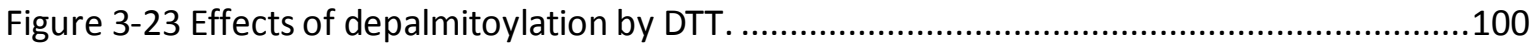




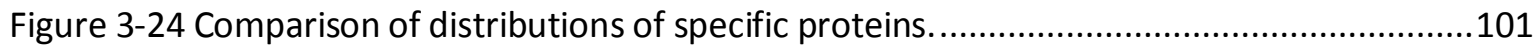

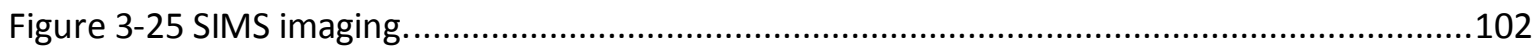

Figure 3-26 Sample preparation for correlative imaging .......................................................104

Figure 3-27 Correlative STED and SIMS imaging in neuronal axons. ........................................106

Figure 3-28 Correlative STED and SIMS imaging in neuronal cell bodies....................................108

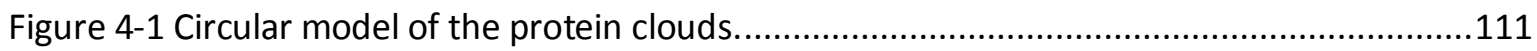

Figure 5-1 Protein clouds as a membrane organizing principle.................................................135

\section{LIST OF TABLES}

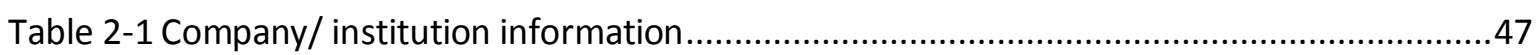

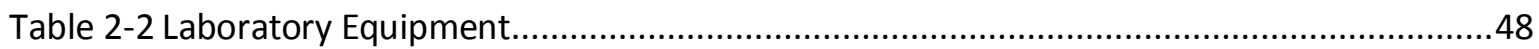

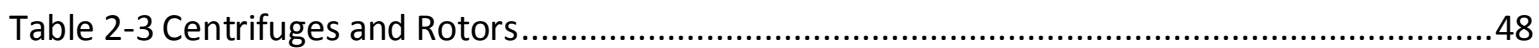

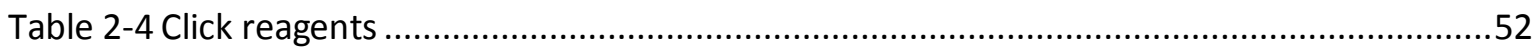

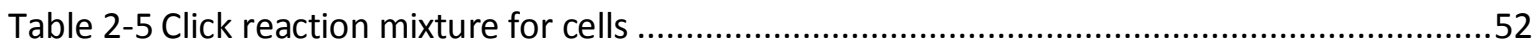

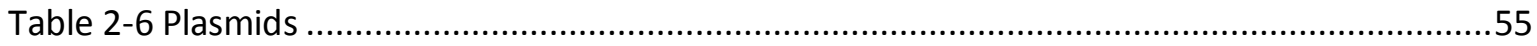

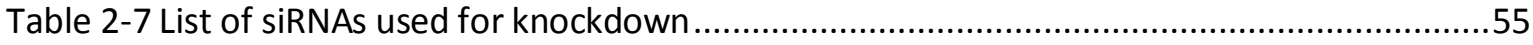

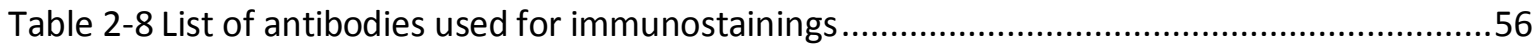

Table 3-1 Summary of all specific proteins investigated............................................................92

\section{LIST OF EQUATIONS}

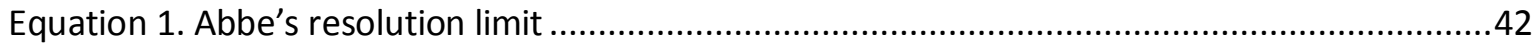

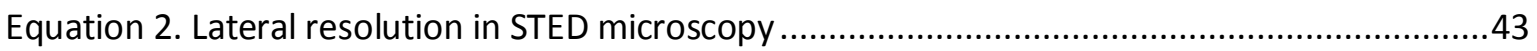




\begin{tabular}{|c|c|}
\hline ACP & Acyl carrier protein \\
\hline $\mathrm{AHA}$ & L-azidohomoalanine \\
\hline AFM & Atomic force microscopy \\
\hline APP & Amyloid precursor protein \\
\hline BACE & $\beta$-secretase \\
\hline BSA & Bovine serum albumin \\
\hline$C D$ & Cyclodextrin \\
\hline Chol & Cholesterol \\
\hline cOase & Cholesterol oxidase \\
\hline COIN & Correlated optical isotopic nanoscopy \\
\hline DDR & Davson-Danielli-Robertson \\
\hline DMSO & Dimethyl sulfoxide \\
\hline EE & Early endosome \\
\hline EGFR & Eukaryotic growth factor receptor \\
\hline EM & Electron microscopy \\
\hline ER & Endoplasmic reticulum \\
\hline ERM & Ezrin/radixin/moesin \\
\hline FCS & Fluorescence correlation spectroscopy \\
\hline FP & Fluorescent protein \\
\hline FPALM & Fluorescence photoactivation localization microscopy \\
\hline FRAP & Fluorescence recovery after photobleaching \\
\hline FRET & Förster resonance energy transfer \\
\hline GalNaZ & Tetraacetylated N-azidoacetylgalactosamine \\
\hline GFP & Green fluorescent protein \\
\hline GlcNaZ & Tetraacetylated N-azidoacetylglucosamine \\
\hline GPI & Glycosylphasphatidylinositol \\
\hline GPMV & Giant plasma membrane vesicle \\
\hline GUV & Giant unilamellar vesicle \\
\hline HPG & L-homopropargylglycine \\
\hline KD & Knockdown \\
\hline$L_{d}$ & Liquid-disordered \\
\hline LDL & Low-density lipoprotein \\
\hline $\mathrm{L}_{\mathrm{o}}$ & Liquid-ordered \\
\hline ManNaZ & Tetraacetylated N-azidoacetyl-D-mannosamine \\
\hline $\mathrm{MBCD}$ & Methyl-beta-cyclodextrin \\
\hline NGF & Neuronal growth factor \\
\hline NSF & N-ethylmaleimide-sensitive factor \\
\hline PALM & Photoactivated localization microsocpy \\
\hline PE & Phosphatidylethanolamine \\
\hline PEG & Polyethyleneglycol \\
\hline PFA & Paraformaldehyde \\
\hline PIP2 & Phosphatidylinositol-4,5-bisphosphate \\
\hline PLL & Poly-L-lysine \\
\hline R18 & Octadecyl rhodamine B \\
\hline RT & Room temperature \\
\hline SDS & Sodium doedecyl sulphate \\
\hline SM & Sphingomyelin \\
\hline
\end{tabular}


Structure illumination microscopy

SIMS

Secondary isotope mass spectrometry

SiRNA

Small interfering RNAs

SNAP

Soluble NSF attachment protein

SNARE

Soluble NSF attachment protein receptor

SPT

Single particle tracking

STED

Stimulated emission depletion microscopy

STORM

Stochastic optical reconstruction microscopy

TfnR

Transferrin receptor

TIRF

Total internal reflection microscopy

$T_{m}$

WGA

Melting temperature

YFP

Wheat germ agglutinin

Yellow fluorescent protein 


\section{INTRODUCTION}

\subsection{A Brief History of the Plasma Membrane and Early Models}

The outer boundary of cells has been a subject of extensive research and discussion since the first microscopic observations of cells and formulation of the cell theory. The timeline of major contributions can be seen in Figure 1-1. Initially cell wall was referred as the "membrane" and was thought to be an indispensable part of the cell (Baker, 1952). Later, it was accepted not to be a necessary constituent of cells, and the focus was shifted to the protoplasm membrane (Leydig, 1857). The first insights about the biophysical characteristics of the membrane, such as semipermeability and viscosity, were derived from studies of colloids and emulsions, pioneered by Lord Rayleigh, Moritz Traube and Otto Bütschli (Butschli, 1892; Loeb, 1906; Rayleigh, 1890). Based on the spherical shape the protoplasm adopts in water and the physical characteristics of the lipid layers, Georg Quincke suggested that the protoplasm should be enclosed by a semipermeable thin film of fat or oil (Quincke, 1888; 1893). Correspondingly, Charles Ernst Overton showed that the alcohols with large molecules were able to penetrate into the cells more readily than small salt molecules (Ling, 1984). The finding that cells showed highest permeability to narcotics like alcohol or ether, which have great solubility lipoids (such as phosphotidylcholine and cholesterin), indicated the presence of a lipoic membrane (Loeb, 1906; Meyer, 1899)

Much of the basic knowledge about lipid layers was obtained through surface tension experiments. Development of Langmuir trough (Figure 1-2A) enabled the properties of lipid layers, such as flexibility or monolayer formation to be analyzed at a quantitative level (Langmuir, 1917; Pockels, 1891). By spreading the lipids extracted from a known number of erythrocytes on a Langmuir trough and calculating the surface area, Gorter and Grendel identified the bilayer nature and thickness of the cell membrane (Gorter and Grendel, 1925). Although the idea of a bilayer was accurate, their technically erroneous measurements were later corrected by the finding that the proteins also significantly contribute to the membrane surface (Bar et al., 1966; Singer, 2004). Based on surface tension measurements and permeability properties, it was expected that there would be an adsorbed layer of proteins on both sides of the membrane (Robertson, 1964). This early membrane model is known as the Davson-Danielli-Robertson (DDR) model (Figure 1-2B). With the introduction of electron microscopy (EM), a $90 \AA$ A-thick "rail-road track" like structure was repetitively seen around different types of cells and organelles. This 
structure was $\sim 2 \mathrm{~nm}$ thicker than the expected $70 \AA$ bilayer and the DDR model attributed this difference to the presence of a protein layer.

Later, images of chloroplast membranes obtained by the newly developed freeze-etch EM displayed a mosaic of repetitive lipoprotein units in contrast to the protein-lipid sandwich of the DDR model. This observation led to the proposal of the lipoprotein subunit model by Andrew Benson (Benson, 1966). In this model, the lipids were thought not to be in a classical bilayer, but completely intertwined with proteins (Figure 1-2C). Conversely, applications of spectroscopic methods demonstrated that $\sim 1 / 3^{\text {rd }}$ of the membrane proteins were found in a nonpolar environment and adopted a helical conformation (Lenard and Singer, 1966). These results suggested that instead of being intertwined with lipids, integral proteins were spanning the entire bilayer (Lenard and Singer, 1966; Wallach and Zahler, 1966). However, the cell surface proteins were still able to diffuse on a global scale demonstrating the fluidity of the membrane (Frye and Edidin, 1970). These observations were integrated together in the famous fluid mosaic model (Singer and Nicolson, 1972). In this model, the proteins integral to the membrane are described as globular molecules arranged in an amphipathic structure, nonpolar groups buried in the interior of the membrane and ionic groups protruding into the aqueous phase (Singer and Nicolson, 1972). The model proposes a viscous phospholipid bilayer to be the solvent for integral proteins, forming a two-dimensional fluid mosaic (Figure 1-2D). Such a membrane structure allows lateral free diffusion of proteins and lipids, whereas the rotational free diffusion is restricted contributing to the membrane asymmetry like in the case of oligosaccharides found only in the outer leaflet (Singer and Nicolson, 1972).

In the meantime, lipid phase transitions of lipid systems and the membrane fluidity were also extensively studied (Edidin, 2003). Different phases were recognized, the main ones being the solid gel and liquid-disordered ( $L_{d}$, also known as liquid-crystalline or fluid). The $L_{d}$ phase was associated with a rather-stable long-range molecular organization assuming a relative disorder at the short range which allows flow and lability (Chapman, 1975). The possibility of different phases co-existing in the membrane and a bilayer asymmetry were proposed to create packing heterogeneities in the membrane with possible effects in the organization and function (Chapman, 1975; Jain and White, 1977). 
1838

1857

1879

1888

1890

1917

1925

1960

1970

1972

1975

Studies of thermodynamic phase transitions of lipids and fluidity characteristics of membranes raised the possibility of packing heterogeneities and lipid domains in the plasma membrane (Chapman, 1975; Jain and White, 1977).

Matthias Schleiden and Theodor Schwann observed plant and animal cells, eventually leading to the cell theory. The cell wall was referred as the "membrane" (Baker, 1952).

Franz Leydig stated for the first time that cell wall is not a necessary constituent of the cell (Leydig, 1857).

Moritz Traube observed the semi-permeable membranes of precipitation by mixing colloids (Loeb, 1906).

Otto Bütschli claimed the living protoplasm to be an emulsion of a water-insoluble viscous liquid and a watery liquid of little viscosity (Bütschli, 1892).

Georg Quincke suggested that the protoplasm is surrounded by a semi-permeable thin film of oil (Quincke, 1888; Quincke, 1893).

Charles Ernst Overton found that the protoplasm membrane is more permeable to alcohols than small salt molecules (Ling, 1984). Based on the oil solubility of narcotics that are readily absorbed by the cells Hans Meyer and Overton suggested that the membrane was made of lipoids (Meyer, 1899; Loeb, 1906).

Agnes Pockels and Lord Rayleigh worked on emulsions and studied the effect of contaminants on surface tension (Rayleigh, 1890). Pockels developed a home-made method to measure surface tension (Pockels, 1891).

Irving Langmuir improved the methodology of Pockels, creating the Langmuir trough (Figure 1-2A) and demonstrated that lipid molecules formed a monolayer on the water (Langmuir, 1917).

Gorter and Grendel proposed the cell membrane to be formed by a lipid bilayer (Gorter and Grendel, 1925).

The Davson-Danielli-Robertson model side (Figure 1-2B): Repetitive visualization of a "rail-roadtrack" like structure in different osmium-stained EM samples created the concept of a "unit membrane" that is composed of a lipoid bilayer lined by proteins in each (Robertson, 1964).

Benson's lipoprotein subunit model (Figure 1-2C): Based on freeze-etch EM images the membrane was suggested to be a mosaic of repetitive intertwined lipoprotein units (Benson, 1966).

Membrane proteins were found to have helical segments spanning the bilayer, creating an understanding for the integral membrane proteins (Lenard and Singer, 1966; Wallach and Zahler 1966).

Immunoelectron microsocpy showed the mixing of cell surface proteins in mouse-human cell fusion heterokaryon. This global diffusion of protein demonstrated the fluidity of the membrane (Frye and Edidin, 1970).

Singer and Nicholson's fluid mosaic model (Figure 1-2D): A viscous phospholipid bilayer is the solvent for integral proteins, forming a two-dimensional fluid mosaic (Singer and Nicholson, 1972).

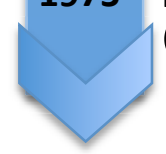

Figure 1-1 Timeline of cell membrane research. 
A

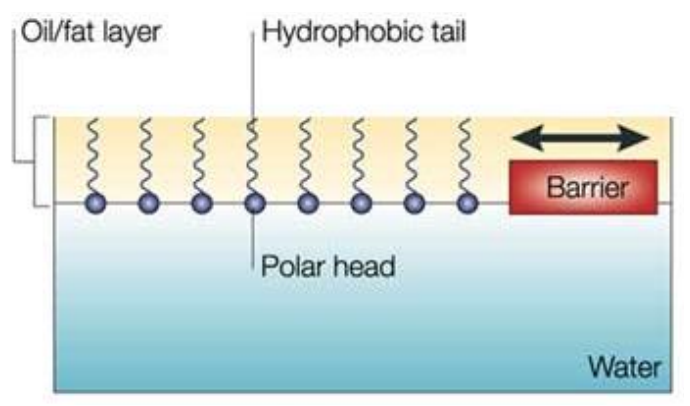

C

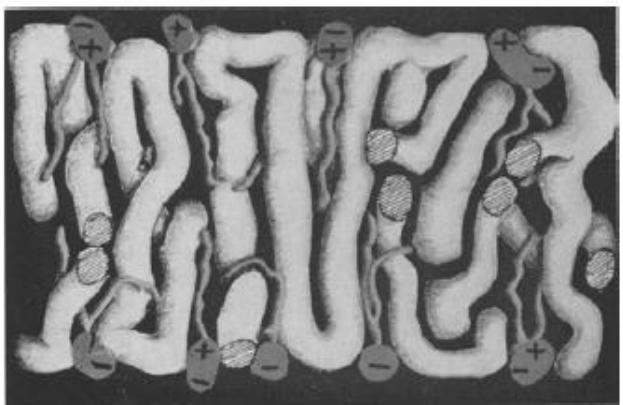

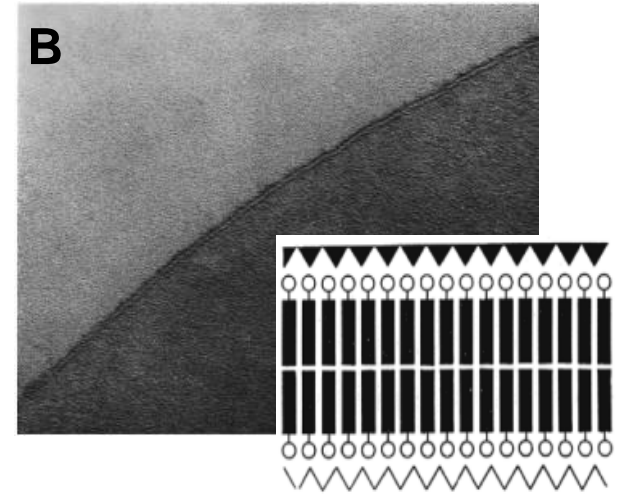

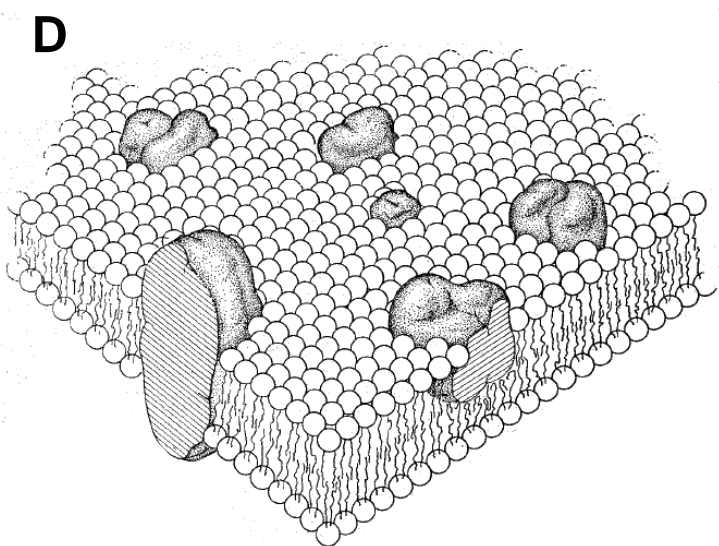

Figure 1-2 Langmuir trough and early membrane models.

A. Langmuir trough. A monolayer of lipid molecules is depicted with polar headgroups facing towards water. The molecule of interest is dropped at one side of the liquid-filled trough. A sliding barrier can be moved to change the surface area available for the spread of the drop, creating a defined pressure on the surface film. The pressure affects the packing of lipid molecules altering the surface tension on the lipid-water interface. B. The electron micrograph of a permanganate-fixed erythrocyte membrane section with osmium-staining, displaying the appearance of the "unit membrane" structure. The graphic on the right represents the DDR model with extended polypeptide chains covering the phospholipid bilayer on each side. C. A depiction of the Benson model. The membrane is composed of lipoprotein subunits that are formed by packing of intertwined proteins and lipids together such that the hydrophobic regions are protected in the center and the charged and polar residues occupy the exterior side. D. The fluid mosaic model with randomly distributed globular integral proteins in the fluid phospholipid bilayer. The panels are reproduced with permission from Edidin, 2003; Robertson, 1964; Benson, 1966 and Singer and Nicolson, 1972, respectively.

\subsection{Composition of the Plasma Membrane}

\subsubsection{The Membrane Lipids}

Cells devote $\sim 5 \%$ of their genes to synthesize thousands of different lipids (van Meer et al., 2008). The structural lipids involved in compartmentalization are generally amphipathic molecules, i.e. they are polar (or hydrophilic) on one end and nonpolar (or hydrophobic) on the other end. Membrane lipids differ in the type of backbone, headgroup, acyl chain length and saturation. They are categorized in three classes (Alberts et al., 2007). The first class is glycerophopholipids, which have a glycerol backbone ester-bound to two fatty acyl tails of varying hydrocarbon lengths 
and saturation. The most abundant glycerophospholipid is phosphatidylcholine (PC, Figure 1-3A). The choline headgroup is as wide as the tails, giving PC a cylindrical geometry. In comparison, phosphatidylethanolamine (PE) has a fairly small headgroup, yielding a conical shape, which creates negative curvature in membranes. Although they have positively charged headgroups, PE and PC do not have a net charge. In contrast, phosphatidylserine (PS) and phosphatidylinositol (PI) have a negative charge. PI constitutes a small fraction of the membrane, but a highly functional one. Reversible phosphorylation of inositol at different positions can produce differentially phosphorylated derivatives like phosphatidylinositol-4,5-bisphosphate (PIP2) and triply phosphorylated phosphatidylinositol-3,4,5-trisphosphate. Local synthesis and removal of these molecules have regulatory effects on signaling events and protein-lipid interactions (Honigmann et al., 2013b; McLaughlin and Murray, 2005).
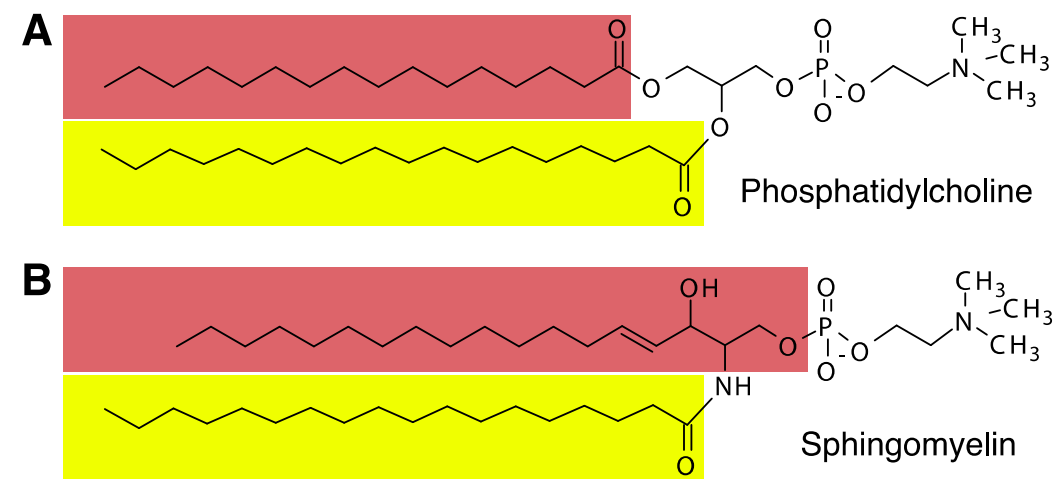

C

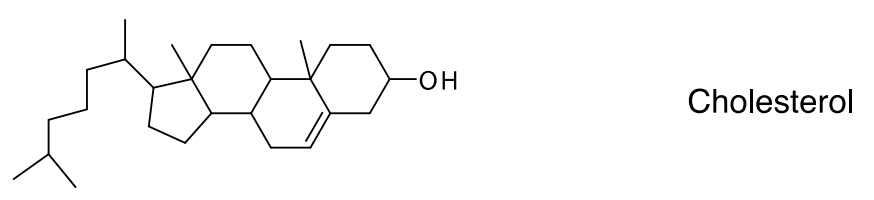

Figure 1-3 Structures of major membrane lipid species.

A. Phosphatidylcholine is the most abundant glycerophospholipid. It is composed of two fatty acid chains (highlighted in pink and yellow) bound to glycerol and the choline head group bound to the phosphatidic acid moiety. B. Sphingomyelin is one of the major sphingolipids. Sphingolipids contain a sphingoid base (pink) amide linked to a fatty acid (yellow). C. Cholesterol is the exclusive membrane sterol of animal cells.

The second class is sphingolipids, which have a sphingosine backbone. Unlike glycerol, sphingosine has a long hydrocarbon chain with two hydroxyl groups and one amino group. Amidelinkage of a fatty acid to sphingosine creates ceramide. Phosphocholine modification of ceramide generates sphingomyelin (SM, Figure 1-3B), which is the major sphingolipid in mammalian cells, accounting for $30 \%$ of the extracellular surface of the plasma membrane on average (Sprong et al., 2001). Sphingolipids mostly have saturated tails, yielding narrow and tall cylinders, allowing them to pack tightly and assume a rather ordered state. Sphingosine-derived lipids can be modified by addition of sugar molecules at their headgroup to form glycolipids (Alberts et al., 2007). These 
sugar groups can vary from a single glucose (glucocerebroside) to complex sugar chains containing sialic acid (as in GM1 ganglioside).

Sterols constitute the third class. These molecules have a planar, cyclic structure and are almost nonpolar. Presence of sterols is a ubiquitous feature of eukaryotic plasma membranes. Fungal cells contain ergosterol and plant cells have phytosterols, whereas cholesterol (Figure 1-3C) is the exclusive structural sterol in animals (Mouritsen and Zuckermann, 2004). The peculiarity of cholesterol as a membrane lipid makes it worthy of a more detailed discussion (as follows).

\subsubsection{Cholesterol}

In mammalian cells phospholipids of an astonishing diversity constitute slightly more than half of the plasma membrane lipids, the rest (accounting for 30-50 \% of the total plasma membrane lipids), in contrast, is mostly made up by one major sterol, cholesterol, which is an indispensible molecule for viability (Ikonen, 2008). It is the single most abundant molecule in the plasma membrane and is structurally quite different. Like phospholipids cholesterol is amphipathic (polar on one side and apolar on the other), however its hydrophobic part consists of a short hydrocarbon tail and a planar steroid ring, giving it a smooth and rigid interaction face. Its polarity comes from a single hydroxyl group at the opposite end, which is exposed at the bilayer surface. Lengthwise, it can span the bilayer only partly. It is produced as the end product of a highly complex pathway in the ER requiring over 30 enzymatic reactions. However, it is found in rather low levels in the ER and gets enriched in the trans-Golgi compartments and at the plasma membrane (Ikonen, 2008). Up to $90 \%$ of the unesterified (free) cholesterol is found in the plasma membrane (Lange et al., 1989). Golgi compartments have an intermediate level, whereas the mitochondria contain the least amount of cholesterol. An alternative route to cholesterol synthesis is the cholesterol uptake from lipoproteins in the circulation through low-densitylipoprotein (LDL) receptor or by direct desorption from the lipoprotein to the plasma membrane (Ohvo-Rekilä, 2002).

The esterified forms of cholesterol (cholesteryl esters, much less polar lipids formed by addition of long-chain fatty acids to the hydroxyl group) are not found in the plasma membrane but are the preferred form for storage and transport of cholesterol.

Cholesterol interacts with phospholipids in a dual mode. On one hand, it favors ordered smooth acyl chains, such as the ones of saturated fatty acids, directing an ordered system. On the other 
hand, it does break when the packing of ordered acyl chains is very tight, as it is conformationally not very compatible. This bimodality has several effects in membranes. Firstly, it leads to stabilization of an intermediate state between the gel and liquid-cyrstalline, the liquid-ordered state $\left(L_{o}\right)$. $L_{o}$ state corresponds to a liquid state with high rotational mobility and lateral diffusion, but with an ordered arrangement of extended acyl chains (Veatch and Keller, 2005). When the bilayer is at a temperature above the phase transition temperature $\left(T_{m}\right.$, the temperature at which the gel to liquid crystal phase transition occurs), cholesterol causes a decrease in fluidity, while it increases the fluidity of ordered chains below the Tm. Second, it has a strong effect on the permeability of the bilayer. By inducing tight packing, it improves the permeability-barrier. Finally, there are many models suggesting distribution of cholesterol in various heterogeneities and cholesterol-driven distribution (or condensation) of other lipids and proteins (Lingwood et al., 2009; Simons and Ikonen, 1997; Smaby et al., 1996; 1994).

Interesting insights into cholesterol-related cellular mechanisms have been provided by cholesterol depletion studies. Cyclodextrins (CDs) are cyclic oligosaccharides formed by starch degradation. They can polymerize at different degrees forming hexamers ( $\alpha$-CDs), heptamers ( $\beta$ CDs) and octamers ( $\gamma$-CDs) that harbor hydrophobic cavities of different sizes. $\beta$-CDs can fit cholesterol in their cavities and are efficient in extracting cholesterol (Ohtani et al., 1989). Therefore, they have been extensively used for acute cholesterol depletion. Especially methyl- $\beta$ $C D(M B C D)$ is preferred for its increased water solubility (Zidovetzki, 2007). Another main alternative for depletion is the use of cholesterol oxidase, which yields 4-choleren-3-one. A longer-term method is metabolic reduction by growing cells in lipoprotein-deficient serum conditions and in presence of inhibitors for one of the enzymes (like statins which inhibit formation of mavelonate) involved in cholesterol synthesis (Shvartsman et al., 2006).

Cholesterol depletion studies established the presence of two distinct kinetic pools of cholesterol at the plasma membrane. The fast pool is removed much more rapidly whereas the slow pool requires more time. However, the mechanism behind the two pools is not clear. Since cyclodextrins can only work on the outer leaflet in the whole-cell experiments, slow pool might be formed by the cholesterol that takes longer time to move from inner leaflet to the outer leaflet or alternatively slow pool cholesterol be found in association with other membrane components making its extraction more difficult (Haynes et al., 2000).

Although there is a broad range of technique-dependent inconsistencies in the previous reports, cholesterol is accepted to flip-flop in the membrane rather fast with a half-time $\leq 1$ second (Steck 
et al., 2002). This fast exchange has been challenging the reliable quantification of transbilayer distribution of cholesterol for different cell types. One study in $\mathrm{CHO}$ cells employed naturally fluorescent sterols like dehydroergosterol and cholestatrienol to quantify the distribution in each leaflet by application of fluorescent quenchers (Mondal et al., 2009). Their addition from outside resulted in the quenching of only 15-32\% of the sterol fluorescence. By comparing the quenching caused by permeabilization of cells or microinjection of the quenchers, $60-70 \%$ of the sterols in the plasma membrane was found to be in the cytoplasmic leaflet (Mondal et al., 2009). These values are in line with fluorescence quenching studies in erythrocytes, which reported $75 \%$ of the fluorescent sterols to be in the cytoplasmic leaflet (Schroeder et al., 1991). Quantifications of the transbilayer distribution in synaptic plasma membrane of neurons indicate presence of up to $85 \%$ of the cholesterol in the inner membrane leaflet (Wood et al., 2011).

In addition to the transbilayer distribution, the lateral distribution of cholesterol in the membrane is also controversial. PALM imaging of cholesterol-binding toxins showed cholesterol-enriched circular or elongated domains width $150 \mathrm{~nm}$ widths (Mizuno et al., 2011). However, distribution of dehydroergosterol, a naturally fluorescent analogue of cholesterol, was shown to follows the cellular topology closely, yielding a homogeneous distribution (Wüstner, 2007). Similarly, recent mass spectrometry imaging experiments with isotopic labeling of cholesterol also indicated a homogeneous distribution (Frisz et al., 2013a).

\subsubsection{The Membrane Proteins}

\subsubsection{Integral Membrane Proteins}

Membrane proteins with bilayer spanning polypeptide chains are called integral membrane proteins (Singer, 1974). It is predicted that $20-30 \%$ of all open reading frames in the human genome code for integral membrane proteins (Wallin and Heijne, 1998). Among integral membrane proteins those that span the bilayer only once are classified as single-pass transmembrane proteins, whereas those that transverse the membrane at least twice are called the multi-pass proteins. In mammalian cells, transmembrane domains are usually $\alpha$-helices made of 18-25 amino acids. Integral membrane proteins can be classified into different groups. From a topology point of view, single-pass transmembrane proteins are called bitopic integral since they have segments exposed to both sides of the membrane and the multi-pass ones are classified as polytopic integral. The other class is the monotopic integral proteins, which do not span the 
bilayer and are presented only at one side. The membrane attachment can be established by insertion of an amphiphilic $\alpha$-helix at one leaflet (Alberts et al., 2007). Another way is the covalent attachment of the protein to a PI in the outer leaflet via a specific oligosaccharide, creating the glycosylphophatidylinositol (GPI) anchor (Vidugiriene and Menon, 1994). Monotopic insertion can also be mediated through covalent modification of the protein with one or more lipid anchors attaching it to the membrane from the cytoplasmic face. The most common anchoring modifications are amide linkage to a $14 \mathrm{C}$ myristate at the $\mathrm{N}$-terminal, thioester linkage to a $16 \mathrm{C}$ palmitate at a cysteine residue, or prenylation of a cysteine through thioether linkage to a $15 \mathrm{C}$ farnesyl or 20C geranylgeranyl (Bhatnagar and Gordon, 1997; Resh, 2006).

\subsubsection{Peripheral Membrane Proteins}

Peripheral membrane proteins do no get inserted at the membrane but are attached to it by interactions with membrane lipids or proteins. Unlike integral membrane proteins, peripheral proteins are released from the membrane relatively easily, as their attachment is usually affected by changes in ionic conditions. They can have domains for interaction with specific lipids or proteins. There are at least 12-conserved lipid binding domains, including well known PH, FERM, PDZ, BAR and FYVE (Scott et al., 2012). The non-covalent interactions of peripheral membrane proteins with the membrane are usually subject to regulation, making them important elements of signaling cascades (Stahelin, 2009).

\subsubsection{Glycosylation of Membrane Proteins}

Majority of the transmembrane proteins are glycosylated through addition of sugar residues in the lumen of the endoplasmic reticulum (ER) and the Golgi apparatus. The type of the glycosylation is determined by the linkage between the first carbohydrate residue in the glycan and the amino acid to be modified (Varki et al., 2009). Usually, carbohydrate chains can be attached to the amide nitrogen of asparagine, leading to $\mathrm{N}$-linked glycosylation in the ER or to the hydroxyl group of serine and threonine through O-linked glycosylation in the lumen of Golgi (Lodish et al., 2008; Mellquist et al., 1998).

\subsubsection{The Membrane Asymmetry}

The complexity of cellular membranes does not only arise from the diversity of its components, but also from their asymmetric distribution. Synthesis and membrane insertion of integral 
proteins occur in a directional manner and once integrated into the membrane they can generally not be transferred across. Post-translational modifications can add another level of asymmetry. Addition of oligosaccharides to proteins is carried out by the enzymes facing the lumen of the ER and Golgi (Varki et al., 2009). This mechanism ensures that glycosylated residues of proteins are facing the exoplasmic surface. Conjugation of glycosylphosphoinositide (GPI) anchor also occurs exclusively at the noncytosolic leaflet, targeting the anchored proteins to the extracellular leaflet. In contrast, other lipid anchors mediate membrane insertion from the cytoplasmic leaflet.

Furthermore, lipids biosynthesis is also vectoral. For example, glycerophospholipids are generally synthesized on the cytoplasmic face of the ER while sphingolipids are rather synthesized or modified at the luminal site of the ER and Golgi (Daleke, 2007). The translational asymmetry generated with synthesis is preserved by the fact that most phospholipids and sphingolipids can cross the membrane bilayer only very slowly at half-times varying with charge, size and polarity of the headgroup. Transbilayer movement can be facilitated by lipid transport proteins such as flippases, floppases and scramblases (Daleke, 2007). However, these proteins are differentially distributed or activated in cellular membranes, meaning that the flip/flop of certain lipids can only happen in the organelles carrying the respective transport protein. As a result of these mechanisms, in eukaryotic cells, PE, PS and PI are enriched in the cytoplasmic leaflet whereas PC, SM and glycosphingolipids are enriched at the outer leaflet (van Meer et al., 2008). Due to accumulation of anionic lipids in the cytoplasmic leaflet, inner face of the membrane is more negatively-charged. Differential interactions of these asymmetrically positioned proteins and lipids with other lipids and the peripheral proteins create a higher level of asymmetry.

\subsection{Modern Models of Membrane Organization: The Lipid Side}

A long way has been traveled since the proposal of the fluid mosaic model; we now know much more about the basic composition of the plasma membrane and the general principles that govern it. However, a precise understanding of the membrane organization, especially at the nano-scale level has still not been gained.

The membranes of living cells are very dynamic and immensely complex (with over thousand different components), making investigations under native conditions rather challenging. Therefore several model systems have been developed to mimic membrane behavior with reduced number of components and within a controlled environment. Although mechanistic relevance of these simplified systems to biological membranes is questionable, they provide 
important insights and have been especially useful to study certain biophysical behaviors such as phase transitions, partitioning or curvature effects. For this reason, most of the work on membrane organization has been performed using simplified systems, such as model membranes. These models vary from suspended or supported mono or bilayers to liposomes of various sizes, containing a limited number of components.

\subsubsection{Phase Separation}

Phases can be formed even in bilayers of a single-component, under conditions of different temperature, pressure or depending on the properties of the solvent such as ionic strength or $\mathrm{pH}$. For example, at low temperatures, the bilayer is in the gel phase, where the acyl chains would be densely packed and less hydrated. With increasing temperature, this order is gradually lost and the bilayer becomes more fluid. In the fluid phase, the mobility increases and the acyl chains become less stretched and packed, creating a thinner and more permeable membrane.

When the number of components in the bilayer is increased, these phase transitions are not as smooth since each of the components has different $T_{m}$ values. So depending on the temperature and concentrations of the components, mixed phases can appear and co-exist (Veatch and Keller, 2005). The coexisting phases could be composed of long-lived, macroscopic patches at equilibrium or separate into microscopic or submicroscopic domains of more transient nature (Veatch, 2007). It is observed that for co-existing micron-scale phases membranes of at leas three-components are necessary (Veatch and Keller, 2005). At the molecular scale, a lipid molecule can partition into a domain depending on the length or saturation of its acyl chains, the form of its headgroup or its interaction with other molecules. Local preferences directed by such properties could create complexes with different characteristics and compositions than the surrounding bilayer, since these complexes can preferentially include or exclude other molecules (Almeida et al., 2005).

In biological membranes, gel phase is not very compatible with the membrane functioning, and fluid phases are more relevant with a possibility of $L_{d}$ and $L_{o}$ phases co-existing. To create a similar situation in model membranes, usually ternary lipid mixtures that contain cholesterol and two lipids of varying $T_{m}$ values are employed (de Almeida et al., 2003). In these systems it is possible to see complex phase diagrams with many transition versions of $L_{o}$ and $L_{d}$ phases co-existing (Veatch and Keller, 2005). In model systems as well as in cellular membranes, cholesterol is thought to be one of the most important determinants for phase behavior of the membrane and partitioning of 
other components. However, even in model systems, a consensus on description of molecular interactions of cholesterol is not reached.

One of the most successful attempts in this regard was the investigations of the lipid phases in plasma-membrane derived vesicles (Baumgart et al., 2007; Levental et al., 2009; Lingwood et al., 2008). It was seen that these structures could undergo cholesterol-dependent large-scale phase separation, which suggested that in all its complexity plasma membrane can still be predominantly governed by the rules of simplified models. However, the large-scale phase separations seen in model membranes or plasma-membrane derived vesicles (Baumgart et al., 2007; Lingwood et al., 2008) have not been observed in the plasma membrane of living cells under normal conditions. One shortcoming is that the extent of membrane composition preservation in GPMVs and the effect of used vesiculants are not very clear, and the membrane skeleton is known to be lost greatly in these structures (Baumgart et al., 2007). For example, Nethylmaleimide, one of the chemicals used to induce GPMV formation, was previously shown to cause a redistribution of cholesterol in the membrane, indicated by doubling of the fast extracting pool of cholesterol (Haynes et al., 2000). In a recent study, It was found that the membrane was composed of a sub-resolution mixture of $76 \% \mathrm{~L}_{0}$ and $24 \% \mathrm{~L}_{d}$ by analyzing the fluorescence lifetime decay of Laurdan (Owen et al., 2012).

\subsubsection{Cholesterol-Lipid Interaction Models}

Based on its physical characteristics, a number of models have been suggested for cholesterol behavior. One of them is the umbrella model. The model is based on the finding that in model membranes cholesterol precipitates from the bilayer mixtures of PC and PE when certain molar cholesterol ratios (respective maximum solubilities) are reached (Huang and Feigenson, 1999). According to this, the phospholipid headgroups at the membrane surface act like umbrellas that shield the nonpolar part of cholesterol (which cannot be completely covered by cholesterol's lone hydroxyl group) from exposure to water. Increasing cholesterol concentration causes the umbrella headgroups to reorient in order to cover more area, causing a tight packing of the acyl chains and cholesterol below the membrane interface (Figure 1-6). Further increases in cholesterol concentration are not energetically favorable as the headgroups cannot stretch and shield more than a certain number of cholesterol molecules (Huang and Feigenson, 1999) The main consequence of this model is that cholesterol-cholesterol interactions are highly unfavorable and at high cholesterol mole fractions, the mixing behavior should be based on avoiding this unfavourable free energy of unshielded cholesterol. 
An important thermodynamic property of cholesterol is the chemical potential (Figure 1-6). Chemical potential is the cost of adding one more cholesterol molecule to a bilayer. This cost seems to be higher in unsaturated lipid bilayers, which suggest that the interaction between cholesterol and saturated chains are more favorable (Bennett et al., 2009). Also steep increases (jumps) in chemical potential are seen at certain molar fraction ratios of cholesterol depending on the other phospholipid component (Ali et al., 2007). For the umbrella model these jumps correspond to the points where more cholesterol cannot be protected under the phospholipid umbrella (Huang and Feigenson, 1999).

The condensed complex model is based on the compaction seen in cholesterol containing model membranes to explain the changes in cholesterol chemical potential (Radhakrishnan et al., 2000). The model elaborates that cholesterol has the ability to form complexes with lipids, causing a condensation in size with smaller lateral area occupation compared to the uncomplexed form (McConnell and Radhakrishnan, 2003). The complexes can be explicitly formed with lipids that cholesterol favors, like those with long saturated chains (Figure 1-6). Through these condensed complexes the chemical potential of cholesterol is decreased and it becomes less available for extraction from membrane or for enzymes to act on (Ikonen, 2008). Here the jumps are defined by the stoichiometric composition of the complexes. However, most studies of condensed complexes are performed on monolayers that might have different behavior than bilayers (Radhakrishnan et al., 2000; 2001).

Another model known as the superlattice model suggests that the small cross-sectional area of cholesterol in comparison to phospholipids can create a long-range repulsion between cholesterol molecules. This model takes this repulsion force as the predominant factor for cholesterolphospholipid interactions and predicts formation of fairly regular (non-random) superlattice distributions (Somerharju et al., 1999). When molar fraction of cholesterol is changed, different arrangement of superlattices are formed, accounting for the jumps in chemical potential (Huang, 2009). The superlattice model partly agrees with the umbrella model for the regular distribution behavior of cholesterol, however different driving forces are proposed (Ali et al., 2007). 


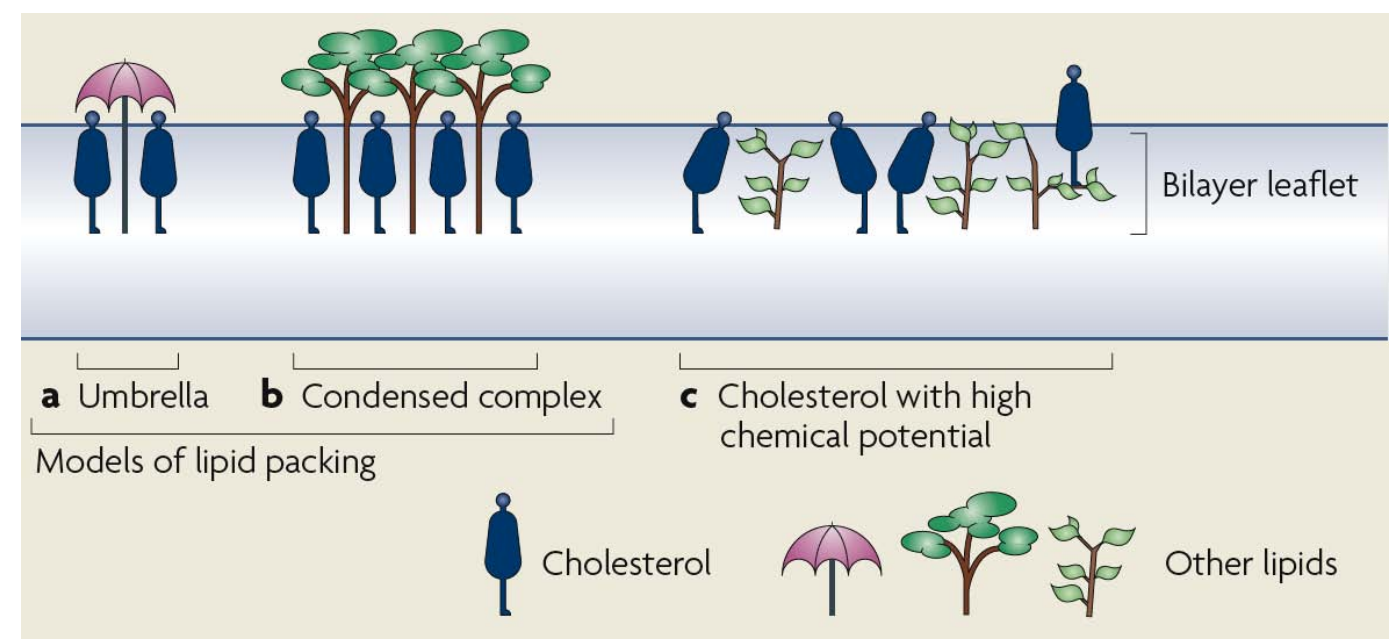

Figure 1-4 Interaction models of cholesterol with other lipids

Graphic representations of the umbrella model, condensed complex model and complex formation dependent changes in the chemical potential of cholesterol are depicted. The figure is reprinted with permission from Ikonen, 2008.

\subsubsection{Sphingolipid Complexes}

Neutral glycosphingolipids such as gylcosylceramides, were found to self-associate and segregate into small domains in phospholipid bilayers of model systems (Thompson and Tillack, 1985). Electron spin resonance spectroscopy studies in mixed bilayers showed that the interaction of cholesterol is stronger with sphingomyelin than with glycerophospholipids (Sankaram and Thompson, 1990). Increasing the negative charge of the headgroup favors greater association with cholesterol, whereas the chain length and chain unsaturation seemed not to have as strong of an effect. In artificial monolayers interaction of cholesterol was found to induce condensation of the area sphingomyelin occupies (Radhakrishnan et al., 2001; Smaby et al., 1996) suggesting a possible association. It was also shown in monolayers that GM1 partitioned exclusively in condensed-complex rich phases (Radhakrishnan et al., 2000). In freeze-fracture immuno-EM experiments with fibroblasts gangliosides GM1 and GM3 in the outer leaflet were found to form clusters that are susceptible to cholesterol depletion (Fujita et al., 2007).

However, there are also contradictory results obtained from artificial bilayers and large unilamellar vesicles arguing against a specific interaction between sphingolipids and cholesterol (Holopainen et al., 2004; Smaby et al., 1994). Cholesterol-induced condensation has been shown for other phospholipids like phosphatidylcholine species in monolayers, indicating that it is not unique to sphingomyelin, and the condensation effect of cholesterol was proposed to be determined by the hydrocarbon chain structure and headgroup together (Smaby et al., 1994). It should be kept in mind that the results obtained in these studies are largely dependent on the 
model system and lipid species or fluorescent analogues used. Importantly, the relevance of either observation to the possible associations in cellular membranes is not very clear. In living cells, different fluorescent sphingomyelin analogs were found in micrometric patches (Tyteca et al., 2010). On contrast, probing of sphingomyelin with the high affinity toxin lysenin at high resolution showed round domains with a diameter of $\sim 120 \mathrm{~nm}$, which were not sensitive to cholesterol depletion (Mizuno et al., 2011). Recently, isotopic imaging of sphingolipids in fibroblasts partly supported this observation. ${ }^{15} \mathrm{~N}$-sphingolipid microdomains with mean diameter of $\sim 200 \mathrm{~nm}$ were observed (Frisz et al., 2013b). However these domains were not enriched with cholesterol, nor eliminated by cholesterol depletion, but were dependent on cortical actin cytoskeleton (Frisz et al., 2013a; 2013b).

\subsubsection{Protein-lipid interactions}

The above-mentioned models of lipid domain formation are mainly based on thermodynamic measurements in model membranes and molecular dynamics simulations, under conditions that proteins were usually ignored. However in cellular membranes proteins typically make $50 \%$ of the membrane by mass (Alberts et al., 2007) and are expected to have significant effects in the properties and organization of the membrane.

An early perspective about protein-lipid interactions came from experiments with spin-labeled fatty acyl chains (Lange et al., 1985). These paramagnetic analogues incorporated into reconstituted membranes with or without protein. Electron spin resonance studies indicated existence of a subpopulation of lipids with decreased rotational mobility, only in protein containing membranes. These less mobile lipids were disordered compared to the rest (Marsh and Horváth, 1998). They are thought to form a shell around the hydrophobic regions of membrane proteins. The shell lipids might be distorted to pack the rigid protein surface, making them conformationally disordered but also restricted in motion. These lipids in immediate proximity of proteins are called the boundary or annular lipids (Lee, 2003).

The lipid annulus concept has been taken to a different level by the lipid shell model (Anderson, 2002). In this model, which can be thought as an extension of the condensed complex model (Section 1.3.1.1), the lipid shells are formed by long-term specific interactions of proteins with condensed lipid complexes. Here the lipid shell is like an extended annulus. Shell formation is a dynamic process that requires complex formation through self-assembly of cholesterol with phospholipids (predominantly sphingomyelin) and the affinity of the protein to the complex. 
Many of the integral or peripheral membrane proteins have specific lipid-binding domains. Furthermore, depending on the structure of proteins, hydrophobic or electrostatic interactions between proteins and lipids might play a role for formation of lipid shells (Anderson, 2002). The shells are not expected to form separate phases but rather make thermodynamically stable mobile entities surrounding the proteins. Around each membrane embedded protein they generate a local environment of the certain size and composition that match the physicochemical requirements of the protein best. The shells can also generate high-affinity interactions with other proteolipidic assemblies, making them important for lateral domain formation.

Principal modes of protein-lipid interactions are reversible association of peripheral proteins to membranes through electrostatic interactions or through the molecular surfaces of the proteins. Certain amphiphilic secondary structures or hydrophobic loops in the protein can mediate their reversible insertion in the membrane. For instance, banana-shaped lipid-binding domains, known as BAR domains, form dimers that insert into the curved membranes assisted by electrostatic interactions or by amphipathic helices (Bhatia et al., 2009; McMahon and Gallop, 2005). Furthermore, more specific lipid protein interactions mediated through lipid interaction domains pose important regulatory mechanisms for functional regulation and localization of proteins. Some protein families contain modular domains specialized in binding certain lipid species. For example, pleckstrin homology (PH) domain found in some signaling or cytoskeletal proteins, such as phospholipase $\mathrm{C}$, dynamin, serine/threonine or tyrosine protein kinases, can specifically bind to PIP2 (Cho and Stahelin, 2005). Also ezrin/radixin/moesin (ERM) family of proteins, which are involved in the membrane association of cytoskeleton, bind the membrane through their FERM domains that interact with PIP2 and its derivatives (Hamada et al., 2000).

When it comes to specific interactions with cholesterol, conserved cholesterol binding domains have been harder to discover. Some members of the cholesterol homeostasis and trafficking machinery possess a conserved sterol sensing domain made up of 5 transmembrane helix domains, although cholesterol binding capability of this domain was demonstrated for only one sterol regulator protein (Ikonen, 2008). In addition to these large hydrophobic pockets, some shorter motifs with cholesterol binding potential have also been identified, such as the cholesterol recognition/interaction amino acid consensus (CRAC) motif found in caveolin 1 (Epand et al., 2005). Although the CRAC domain seems to promote the interaction with cholesterol, the consensus sequence is quite broad and it does not implicate a specific 1:1 interaction with cholesterol (Epand, 2008). 
Interactions with lipids or the lipid environment can have significant effects for the conformation and functioning of proteins. For example ganglioside GM3 was found to specifically inhibit the kinase domain activity of the eukaryotic growth factor receptor in a phase-separation sensitive manner (Coskun et al., 2011). In particular cases lipids are so tightly associated to proteins that they have been identified as part of the crystal structures (Coskun and Simons, 2011). These lipids that are found buried in the binding sites between the hydrophobic segments of the proteins are referred to as non-annular lipids; they can contact with the lipid annulus and help stabilize the protein conformation in the membrane (Lee, 2011).

\subsubsection{Hydrophobic Mismatch}

The concept of hydrophobic mismatch refers to a mismatch between the length of the hydrophobic segment of proteins and the thickness of the hydrophobic core of the lipid bilayer (Mouritsen and Bloom, 1984). To overcome the mismatch some adaptation can happen for both lipids and proteins. Flexible acyl chains of the lipids can adopt a more ordered or more disordered arrangement to change the thickness. Changes in membrane curvature can also modulate the bilayer thickness. The proteins can undergo a conformational change, or change the orientation of their hydrophobic segment by tilting it or by exposing it to the surface (Killian and Nyholm, 2006). One way to change the orientation of the membrane spanning domains is palmitoylation. In other cases proteins can oligomerize or aggregate to minimize the exposure to lipids (Killian, 1998). However, these adaptations are costly in energy, so it is expected that proteins will prefer the areas of the lipid bilayer whose thickness match the length of the membrane spanning sequences.

The bilayer thickness is determined by the lipid composition. Long hydrocarbon chains and ordered chain arrangements increase thickness. Cholesterol is also known to cause thicker and stiffer membranes (Simons and Sampaio, 2011). When the relative increase of cholesterol from ER to plasma membrane is considered, the hydrophobic mismatch could be involved in sorting of proteins and formation of domains in different organelles (Mouritsen, 2011). This change actually coincides quite well with the increasing length of transmembrane domains of integral proteins along the secretory pathway (Sharpe et al., 2010). 


\subsection{Modern Models of Membrane Organization: The Protein Side}

Domains come in different flavors; on one edge are the lipid domains in model membranes, which are formed purely on thermodynamics; in the other edge are other functional nanodomains such as specific receptor clusters, which are formed through a complex set of interactions and with a functional basis (Edidin, 2001). Although formation of the latter cannot be uncoupled from the first, lipid and protein domains can be generally categorized based on the perspective and the experimental system. Here, protein domains refer to membrane domains that include proteins and that are not formed solely on lipid-lipid interactions. Some of the lipid domain models can be adapted to account for the presence of proteins and be predictive of possible protein-lipid interactions. However, to study interactions that include proteins, it is also necessary to experiment on more complex membranes that are either mimicking the main properties of the proteolipid membranes or are derived from cellular membranes directly (Simons and Gerl, 2010).

\subsubsection{Model Systems and Techniques}

\subsubsection{Experimental Systems}

The simplest of these models are obtained by reconstitution of selected protein species to liposomes and or supported/suspended bilayers. Liposomes can be of different sizes and ipid compositions. The commonly used giant unilamellar vesicles (GUVs) are 10-100 $\mu \mathrm{M}$ (Sezgin and Schwille, 2012). These are especially useful to study the domain formation behavior or lipid interactions of a certain protein and the curvature mechanisms under controlled conditions. A more elaborate biological system is giant plasma membrane vesicles (GPMVs) (Scott, 1976; Sezgin et al., 2012a). These are micrometer scaled blebs produced from the cell membrane by treatment with chemical vesiculants such as paraformaldehyde (PFA) and dithiothreitol (DTT) or N-ethylmaleimide (NEM) (Baumgart et al., 2007). They have the full complexity of the plasma membrane, however most of the cytoskeleton was shown to be excluded from the blebs (Baumgart et al., 2007).

Plasma membrane spheres are principally similar to GPMVs, but are obtained by swelling of the cells, which causes formation of a spherical membrane outgrowth from the cell surface, having a diameter of 25-30 $\mu \mathrm{m}$ (Lingwood et al., 2008). Although these larger spherical systems are good 
for fluorescence imaging, their spherical shape makes them not very ideal especially for highresolution imaging.

An alternative is to obtain flat, inside-out patches of plasma membrane (membrane sheets) by shearing the cells physically (Avery et al., 2000). The shearing can be done in various ways, by applying a brief sonication pulse (Lang et al., 2001), or by hypotonic (Frick et al., 2007) or mechanical rupture (Lillemeier et al., 2006). It is necessary that the membrane is well-attached to a flat surface so that it is not lost after the shearing. Plasma membrane sheets have the advantage of presenting the full complexity of the membrane in the absence of other cellular constituents. Most of the time, peripheral membrane proteins, cytoskeletal elements and docked vesicles are still visible in the sheets. Using membrane sheets it was even possible to study endo-/exocytosis of secretory granules (Holroyd, 2002; Lang et al., 2001). Being thin and flat, they offer a possibility for higher-resolution and crisper images, since there is not out of focus fluorescence that can cause blurring. Because they are inside out, the cytoplasmic face is available for direct treatments or for labeling (without the need for permeabilization). Of course this also creates disadvantages such as loss of some components due to ease of extraction or aggregation of proteins over time (Frick et al., 2007): therefore, for this system it is beneficial to use stabilizing buffers or employ fixation protocols.

Being one of the easiest cellular materials to isolate, plasma membrane of red blood cells (erythrocytes) has been the most extensively studied eukaryotic membrane. Osmotic hemolysis of erythrocytes makes them permeable, so the intracellular material can be washed out, leaving the resulting membrane behind, the so-called ghost. The basic cell shape is retained in the ghosts as the submembraneous cytoskeleton persists during this treatment. The major protein of the ghosts is spectrin, which forms a rigid scaffold beneath the membrane. In this permeable form both leaflets are accessible for treatments with reagents. Alternatively, the membrane can be allowed to reseal to study the two leaflets separately. It is also possible to obtain inside-out vesicles from the ghosts (Alberts et al., 2007).

Erythrocyte ghosts have been extensively useful for biochemical analyses and for investigations of membrane composition and protein orientation, as they make it possible to assay the plasma membrane without the contamination of cytoplasmic material and membrane-bound organelles (Schroeder et al., 1991). One problem with erythrocyte ghosts is that different preparation conditions produce ghosts with different properties, so the results obtained with are methoddependent. Another problem is the possible differences of erythrocytes compared to other cells. 
For example, the cytoskeleton of most cell types is not as rich in spectrin and as robust as that of erythrocytes (Pumplin and Bloch, 1993).

Expectedly, none of these can replace a fully functional living cell. Nowadays, it is becoming easier to perform experiments with living cells from a variety of cell lines. However, depending on the particular question it might be too complicated or even impossible to perform some measurements in living cells. In these cases, one of the more simplified systems above is preferred. In these systems apparently the dynamic regulation of the membrane and material flow mechanisms are lost along with some possible components. However, they can still provide snapshots of the membrane and give hints about how it might respond under different conditions while enabling more controlled experiments with less elaborate techniques. This is the reason why these systems are still employed much more often than living cells for studies of membrane organization.

\subsubsection{Experimental Techniques}

In the last two decades, a plethora of imaging based techniques has been increasingly engaged in studies of membrane patterning to complement the standard biochemical assays. One of the older techniques used to study the diffusion of membrane components is fluorescence recovery after photobleaching (FRAP). In FRAP experiments, a certain population of the fluorescently labeled molecules are bleached and the fluorescent recovery as a result of the movement of new molecules into the area (Kenworthy et al., 2004) is measured. This way, macroscopic diffusion coefficients and mobile fractions can be determined. A drawback of FRAP is that it can only follow the movement of a large population in the bleached area, instead of individual molecules. For example, in cases where the fluorescence does not recover it cannot differentiate between possible reasons such as true immobilization or confined motion (Alberts et al., 2007).

An alternative method that addresses this problem is single particle tracking (SPT), where a small number of molecules are tagged and microscopically tracked (Ritchie and Kusumi, 2003). Analysis of particle trajectories presents different modes of movement and the related motion parameters. Hence, SPT offers a way for measuring microscopic diffusion coefficients (Kusumi et al., 1993). However, it is hard to perform single particle studies at high temporal resolution by using single fluorophores due to low signal-to-noise ratio. Therefore colloidal gold particles are commonly used to probe different molecules allowing up to $25 \mu$ s time resolution (Kusumi et al., 2010). However, these particles are $20-40 \mathrm{~nm}$ in diameter and are usually conjugated through antibodies. 
It is possible that they affect the diffusion behavior. For example it is shown that for an unsaturated phospholipid molecule probing with $40 \mathrm{~nm}$ colloidal gold gave 2.5 times slower diffusion coefficients compared to the fluorescent probing with a small dye (Fujiwara et al., 2002). However this effect is seen rather at longer time windows (seconds) and is not as pronounced for time scales shorter than $100 \mathrm{~ms}$. For the fluorescence tracking at high axial resolution particle SPT method can be combined with total internal reflection (TIRF) microscopy. Also other superresolution microscopy methods are being increasingly employed to study membrane organization. The issue of spatial resolution and basic principles of far-field super-resolution techniques are discussed below in Section 1.5.

Additionally, Förster resonance energy transfer (FRET) is a technique that allows indirect probing of interactions at scales below resolution lipid. FRET is the nonradiative transfer of energy from an excited donor to a nearby acceptor. The FRET effect can be assayed either by the decrease in donor emission or the increase in acceptor fluorescence. The typical donor-acceptor distances for efficient FRET is 5-7 nm, offering a measure for the close proximity interaction between homo or hetero molecules at high spatial sensitivity (Klotzsch and Schütz, 2012). This way FRET can provide information about the clustering behavior of different molecules. However, in addition to simple clustering of tagged molecules, the degree of FRET can also be influenced by other factors such as the relative orientations, rotational mobilities or spectroscopic properties of the donor and acceptor (Lagerholm et al., 2005). Also, clustering-related factors like the cluster size, cluster density, number of donor and acceptor per clusters may have complex consequences in the FRET efficiency, making the results hard to interpret and compare (Kenworthy, 2008).

Another method for diffusion-based studies is fluorescence correlation spectroscopy (FCS). Fluctuations of fluorophores within a confocal laser spot can be analyzed and information about the diffusion and oligomerization of molecules can be obtained (Lagerholm et al., 2005). Also by varying the FCS beam size, it is possible to determine the transition time of the molecule through the illumination spot versus the beam area. This gives the FCS diffusion law that defines the diffusion behavior and the mode of motion of the molecule (Lenne et al., 2006; Wawrezinieck et al., 2005). In this regard, it is complementary to SPT measurements. However, due to diffraction of light, the beam size cannot be made smaller than the resolution limit (see Section 1.5 for a brief discussion about resolution), blocking the measurements at small beam sizes relevant to nanodomains. With the application of STED technique to FCS studies, this limitation has been largely overcome. STED-FCS can decrease the spot diameter down to $30 \mathrm{~nm}$ allowing detection of single molecules diffusing in nanosized membrane areas (Mueller et al., 2013). Remarkably, 
STED-FCS measurements showed differences in the motion of different lipids that cannot be distinguished with confocal FCS (Eggeling et al., 2009).

\subsubsection{Examples of Specialized Protein Domains in Cellular Membranes}

One of the first and most-studied domains in the plasma membrane is caveolae. These cave-like indentations were first seen with EM in the gall bladder epithelium of mouse (Yamada, 1955) and later in many other cell types. These pits generally appear to be uncoated (Parton et al., 2006), however under certain preparation conditions a spiral coat made up of filaments can be visualized (Rothberg et al., 1992). This coat is formed by a protein called by caveolin 1 (Rothberg et al., 1992), which is necessary for caveolae formation. Cholesterol is also critical for formation and maintenance of caveolae. In early freeze-fracture EM studies, it has been detected at the rims of the caveolae (Simionescu et al., 1983), although it was generally seen to be absent in plasma membrane invaginations in general (Montesano et al., 1979). Caveolin 1 seems to have a strong binding affinity to cholesterol (Murata et al., 1995), while the exact interaction mechanism is still not completely understood (Parton et al., 2006, also see Section 1.2.1.1). In addition, a recent molecular dynamics simulation suggested that even in the absence of a direct binding domain caveolin 1 would have a preference for cholesterol-rich bilayers, which can stabilize caveolin conformations required for generation of membrane curvature (Sengupta, 2012). What is clear is that cholesterol depletion causes caveolae to flatten (Rothberg et al., 1992) and increases the caveolin 1-GFP mobility in the membrane (Thomsen et al., 2002). The pits can elongate into flaskshaped invaginations that are internalized and the caveolae domains are known to be important mediators of endocytosis and signal transduction. Interestingly, many proteins are found to be associated to these domains, such as GPI-anchored proteins or some receptors. Although there are controversial views about their biogenesis and composition, the recent view is that stable domains that are mainly constituted of caveolin 1 and possibly cholesterol are formed in the late Golgi compartment and trafficked to the cell membrane directly (Parton et al., 2006).

The small Ras GTPases are another group of well-studied proteins. They are prominent signaling molecules that are activated through GTP binding. All three isoforms $(\mathrm{H}-, \mathrm{N}-$ and $\mathrm{K}$-Ras) are farnesylated (Hancock et al., 1989). Additionally, N-Ras undergoes palmitoylation and H-Ras undergoes double palmitoylation (Hancock et al., 1989; Resh, 2006). K-Ras harbors a polybasic domain at the C-terminal instead. The isoforms have differences in their clustering behavior, signaling capabilities and in their ability to activate various targets (Hancock, 2003). In membrane sheets, all isoforms were found in clusters irrespective of their activation state. In the GDP-state, 
$\mathrm{K}$-Ras clusters are predominantly insensitive to cholesterol depletion, whereas half of $\mathrm{H}$-Ras clusters show sensitivity (Hancock, 2003; Prior et al., 2003). Interestingly activated H-Ras and KRas are localized to distinct plasma membrane microdomains that are both not very sensitive to cholesterol depletion (Prior et al., 2003) and lipid anchoring seems to be an important determinant for correct micro-localization (Plowman et al., 2005).

Besides Ras and caveolae, cellular membranes contain many other types of specialized protein domains or clusters (Lang and Rizzoli, 2010; Saito and Yokosuka, 2006; Simons and Toomre, 2000). An interesting feature of the plasma membrane is that in comparison to artificial membranes diffusion of both proteins and lipids are 5-50-fold slower in the plasma membrane (Fujiwara et al., 2002; Jacobson et al., 1987; Kusumi et al., 2005). In a 1987 review, the main factors proposed to explain slower diffusion of membrane components were molecular crowding and protein density, membrane viscosity, interactions with the cytoskeleton and interactions with the extracellular matrix (Jacobson et al., 1987). After a quarter century, some of these factors formed the bases of major membrane organization models, which explain the heterogeneities and the slowed diffusion in the membrane from different perspectives.

\subsubsection{Membrane Skeleton: Picket-Fence}

In 1980, FRAP was applied to normal erythrocytes and spherocytic erythrocytes that lack the cortical cytoskeletal matrix components to reveal that diffusion of Concanavalin A-probed glycoproteins was 50 times faster in the absence of the cytoskeleton (Sheetz et al., 1980). Later optical trapping was used to drag the immunogold-labeled transmembrane or GPI-anchored major histocompatibility complex proteins until a barrier was encountered (Edidin et al., 1991). Barrier-free path lengths of $0.6 \mu \mathrm{m}$ were measured for transmembrane and $1.7 \mu \mathrm{m}$ for GPIanchored proteins at room temperature (RT). At higher temperatures, 5-fold longer paths were obtained for both. These results suggested presence of cytoskeleton-based compartments.

In an earlier SPT experiment with 33 ms temporal resolution, integral membrane receptors, Ecadherin, transferrin receptor (TfnR) and eukaryotic growth factor receptor (EGFR), were probed with colloidal gold particles conjugated to antibodies or ligands (Kusumi et al., 1993). Different modes of motion were identified by plotting the mean square displacement over time. These modes include a stationary mode (with microscopic diffusion coefficients $<460 \mathrm{~nm}^{2} / \mathrm{s}$ ), simple Brownian diffusion, directed diffusion, and confined diffusion. The confined diffusion was attributed to the presence of membrane compartments created by the membrane cytoskeleton 
(Sako and Kusumi, 1994). While Brownian motion was observed within a compartment, intercompartmental "hops" (hop diffusion) were necessary for the long-range diffusion. Later, a fifth mode was added to this list: diffusion in a harmonic-like potential (like bouncing off flexible borders) due to anchorage to an elastic cytoskeleton (Kusumi and Sako, 1996).

The membrane-skeleton fence model (Kusumi and Sako, 1996) was suggested based on the results of these FRAP and SPT-based diffusion studies of membrane molecules (Kusumi et al., 1993; Sako and Kusumi, 1994; 1995) and the previous work on membrane skeleton (Edidin and Stroynowski, 1991; Edidin et al., 1991; Sheetz et al., 1980). This model stated that cytoskeleton found at close proximity to the membrane creates steric hindrance for the cytoplasmic portions of the membrane proteins, forming an elastic diffusion barrier. Thus, membrane skeleton compartmentalizes the membrane into small domains that confine single molecules and lead to the confined and hop diffusion pattern. Later they have added the transmembrane proteins as pickets to the fence (Fujiwara et al., 2002). The SPT measurements showed that even lipids and GPI-anchored proteins at the outer leaflet undergo confined and hop diffusion, although they are not directly confined by the cytoskeleton at the cytoplasmic side (Dietrich et al., 2002; Fujiwara et al., 2002). This was explained by the presence of immobilized and cytoskeleton-anchored transmembrane proteins, which act like pickets along a fence, transmitting the boundary effect of cytoskeleton to the outer leaflet (Fujiwara et al., 2002). This model commonly known as the fence and picket model is one of the most prominent models in the membrane organization (Ritchie et al., 2003).

In the last two decades, Kusumi and colleagues have contributed SPT data for many molecules at different temporal resolutions (varying from $33 \mathrm{msec}$ to $25 \mu \mathrm{sec}$ ) and time windows (Fujiwara et al., 2002; Murase et al., 2004; Tomishige and Kusumi, 1999). Hop diffusion was observed for all the proteins and lipids that have been tracked (Kusumi et al., 2005). They have validated the picket-fence model on a variety of cell types based on the effects of actin-disrupting drugs, although in each cell type confinement zones of different sizes were found (Murase et al., 2004). Three-dimensional reconstructions by electron tomography enabled visualization of the actin meshwork at the membrane interface (within $10.2 \mathrm{~nm}$ ) forming the membrane skeleton (Morone et al., 2006).

The current state of the picket-fence model views the membrane skeleton, which forms compartments of 40-300 nm diameter, as the first level of organization hierarchy (Kusumi et al., 
2011). This is followed by formation of small raft domains within the compartments and oligomerization of membrane proteins to form complexes.

Recently, a different and more dynamic role of actin was proposed for formation of membrane nanoclusters (Gowrishankar et al., 2012). In addition to the static cortical actin meshwork, this model also puts forward the existence of short, dynamic actin filaments at the cortex and categorizes the proteins into 3 groups based on their interactions with these filaments. The "inert" proteins interact only with the static meshwork but not with the dynamic filaments. Passive molecules interact with the filaments but do not influence them, and active molecules not only interact with the filaments but also have a direct effect on them.

\subsubsection{Membrane Rafts}

In 1990's there were many reports about the poor solubility of GPI-anchored proteins by nonionic detergents. After cold detergent treatment of cells, these proteins were found to associate with a low-density membrane fraction (Brown and Rose, 1992; Schroeder et al., 1994). This low-density, detergent-insoluble fraction (now commonly referred to as detergent-resistant membranes, DRMs) was found to include several proteins like caveolin (Murata et al., 1995) or hemagglutinin (Skibbens et al., 1989) beside GPI-anchored proteins. Based on their detergent insolubility, DRMs were predicted to be mainly in the $L_{o}$ phase (Ahmed et al., 1997). In line with that, they showed an enrichment in sphingolipids and cholesterol in comparison to glycerophospholipids, suggesting that their composition was quite similar to that of apical plasma membrane of intestinal epithelia (Brown and Rose, 1992).

The epithelia tissue lining the body cavities is made up of a single layer of polarized cells. These epithelia cells are exposed to two different environments at each side, the apical side faces the lumen of the cavity whereas the basolateral side contacts the blood supply and the extracellular matrix (Simons and Fuller, 1985). The apical membrane and the basolateral membrane are also different from each other compositionally. In intestinal epithelial cells, the apical membrane is rich in sphingomyelin and glycosphingolipids, and poor in phosphatidylcholine compared to the basolateral. These lipids are mainly localized to the exoplasmic leaflet of the membrane. However even for different polarized cells the relative enrichment is quite variable and the compositional differences for lipids are not that clear (Simons and Fuller, 1985). Nonetheless, for some proteins enrichment at one side seems to be much more significant in comparison to the lipids. One example is TfnR, which shows over 41-fold higher surface density on the basolateral surface 
(Simons and Fuller, 1985). Conversely, GPI-anchored proteins are enriched on the apical membrane (Cao et al., 2012). One important factor for maintenance of the membrane in this polarized composition is the presence of tight junctions at the intersection between apical and lateral membranes, restricting the diffusion of membrane constituents. What is more interesting is the establishment of the membrane polarity and studies along this line have also contributed greatly to the understanding of membrane trafficking and also membrane domains.

To explain polarized delivery to the apical domain, glycosphingolipid domains were proposed to form at the exoplasmic leaflet of the Golgi membrane, acting as sorting centers which concentrate proteins to be targeted to the apical membrane (Simons and Fuller, 1985; Simons and van Meer, 1988; Simons and Wandinger-Ness, 1990). This way, the lipid and proteins were sorted into carrier vesicles that transport them to the correct cell surface membrane. Many observations supported this proposal, like sorting of GPI-anchored proteins (Brown and Rose, 1992; Rothberg et al., 1990) and viral proteins (Müsch et al., 1996; Yoshimori et al., 1996) and the glycosphingolipid/cholesterol-dependent formation of caveolar domains (Parton et al., 2006).

This proposal led to the lipid-raft hypothesis(Simons and Ikonen, 1997). According to this model, lipid rafts are formed by self-association of sphingolipids in the exoplasmic leaflet. Since the sphingolipid headgroup occupies a larger area than the typically unsaturated fatty acyl chain, spacers are needed for tight packing. Planar cholesterol molecules in both leaflets act as spacers and fill the gaps between the acyl chains of sphingolipids. The complementary cytoplasmic leaflet is expectedly composed of phospholipids with saturated hydrocarbon tails. Lipid rafts can recruit specific sets of proteins such as GPI-anchored or doubly acylated proteins or some transmembrane proteins, and exclude others. Individual lipids can move in an out of these sphingolipid-cholesterol clusters, making them hard to detect (Simons and Ikonen, 1997). Lipid rafts were also proposed to be involved in many cellular functions, including membrane trafficking, sorting and signaling (Simons and Ikonen, 1997).

The raft concept was also explaining the DRMs formed after cold Triton X-100 treatment (Harder and Simons, 1997; Simons and Ikonen, 1997). Based on the similarity in composition and assembly of DRMs, it was highly likely that they were originating from the lipid rafts (Harder and Simons, 1997; Simons and Ikonen, 1997) Although, DRMs were essentially viewed as consequences of lipid rafts, together with lipid raft model, concerns about the detergent extraction were also raised (Simons and Ikonen, 1997). Detergent extraction does not show the original cellular locations of DRMs, as well as their presence in living cells. Also finding two proteins in DRMs cannot be an 
indication of their interaction in the cell (Harder and Simons, 1997; Simons and Ikonen, 1997). Still, the proteins found in DRMs are generally accepted to be raft-interacting proteins and for years this method has been used to identify a plethora of proteins that associate with the lipid rafts (Blonder et al., 2004).

Yet, over the years the terms lipid raft and DRMs have been used so often, regardless of the variation in the conditions and methods of to describe domains seen under various conditions and with numerous techniques, what a "raft" means was not really clear any more. To create some consistency in the field, scientists had to gather in a Keystone Symposium and agree on a broad, extended definition (Pike, 2006): “Membrane rafts are small (10-200 nm), heterogeneous, highly dynamic, sterol- and sphingolipid-enriched domains that compartmentalize cellular processes. Small rafts can sometimes be stabilized to form larger platforms through protein-protein and protein- lipid interactions."

The definition makes a distinction between the microdomains in cells and the domains in the model membranes (which are formed by an overlapping, but yet different set of rules), by applying only to the first (Pike, 2006). Membrane rafts include proteins as active participants of the microdomain formation and exclude domains formed solely by lipid-lipid interactions.

After years of research, the behavior of raft phases in model systems is very well established and the potential raft proteins are identified by large scale lipid raft proteomics (Foster, 2008). In general, owing to the presence of cholesterol and high amounts of sphingomyelin, rafts are anticipated as $L_{o}$ membrane phases giving them higher viscosity compared to the surrounding $L_{d}$ phase (London and Brown, 2000). However, the biological extents of cholesterol/sphingomyelin complexes and phase segregation are still controversial (Munro, 2003; Shaw, 2006). In a recent study, degree of lipid packing in the membrane of living cells was addressed by imaging the fluorescence lifetime decay of an environment-sensitive lipid dye called Laurdan (Owen et al., 2012). It was found that the membrane was composed of a sub-resolution mixture of $76 \% \mathrm{~L}_{\circ}$ and $24 \% L_{d}$ domains. This observation is in line with the previous data from model PC:cholesterol bilayers showing that at cholesterol concentrations above $25 \mathrm{~mol} \%$, a more uniform $\mathrm{L}_{0}$-like phase is seen rather than large coexisting phases (Thewalt and Bloom, 1992), and at even higher concentrations (50 mol \%) cholesterol antagonizes lateral phase segregation (Silvius et al., 1996). Having a cholesterol concentration in between these two levels, plasma membrane was proposed to be largely $L_{0}$, with cholesterol stabilizing the boundaries between microdomains (Hao et al., 2001). A largely $L_{o}$ membrane partly contradicts with the view that equates rafts to ordered 
microdomains, suggesting a more complicated scenario (London, 2005). In any case, it is also accepted that in the full complexity of a living cell, there is no standard $L_{0}$ membrane raft but countless number of variations (Munro, 2003; Pike, 2009; Shaw, 2006).

Apart from the lipid order, one immediate outcome of the sphingolipid-cholesterol rich rafts is the expected thickness of such domains. The increased thickness of rafts might be important for formation of platforms that segregate and sort proteins based on hydrophobic matching of their membrane spanning domains (Sharpe et al., 2010; Simons and Sampaio, 2011).

\subsubsection{Protein Densities, Domains, Islands}

Synaptic vesicle is a trafficking organelle in neuronal cells, which undergoes cycles of plasma membrane fusion and retrieval. Its composition has been very well characterized. For an average synaptic vesicle, $67.5 \%$ of the estimated total mass was found to be contributed by proteins (Takamori et al., 2006). This measurement can be in principle representative of the plasma membrane. In another study, shaving of erythrocyte ghosts demonstrated that $>23 \%$ of the membrane area was occupied by transmembrane segments of proteins (Dupuy and Engelman, 2008). This is expectedly an underestimate as there are probably more proteins that might be destabilized and lost during the procedure. When the perturbation of the membrane by the flanking protein segments and by the proteins bound at the surface is also considered, it can be imagined there is little room for the lipids to exist in a state similar to pure bilayers (Dupuy and Engelman, 2008). This means that even the simple presence of a high density of proteins in the membrane can introduce important organizational aspects which are absent in model membranes, underlining the necessity of considering the full protein complexity of the membrane more comprehensively.

An early experiment investigating plasma membrane domains was performed on living fibroblasts by using anti-human cell Fab fragments conjugated to fluorophores (Yechiel and Edidin, 1987). Making gradual changes in the magnification, areas of different sizes were bleached and the fluorescence recovery was observed. They have detected an inverse relationship between the labeling intensity and protein diffusion, suggesting that the membrane consists of protein-rich and protein-poor areas. In support of this observation, a simulation of proteins in single-phase membranes predicted that protein density fluctuations influenced by nonspecific protein interactions can lead to significant protein heterogeneity in the membrane creating sub-micron scale domains (Abney and Scalettar, 1995). Implications of this model were presented by single 
particle trajectories. The confined diffusion of membrane proteins observed by SPT (explained in 1.4.3) is generally attributed to the transient confinement zones, formed by actin fences. However, an alternative explanation is also put forward (Destainville et al., 2008b). The inverse proportion between the microscopic diffusion coefficient and the local protein concentrations was explained by interprotein interactions (Daumas et al., 2003). As an alternative to the picket fence model, the SPT data was fit to a different model, called cluster phase. The idea comes from the physics of colloidal suspensions; proteins are represented by the colloid particles that are found in small clusters (Destainville, 2008). Cluster size is proportional to colloid concentration, whereas the diffusion coefficient of colloids is inversely proportional to both. Based on this, protein clusters in cells might be formed by non-specific short-range attractions and longer-range weaker repulsions (Destainville et al., 2008a).

The role of inter-protein interactions versus raft partitioning was further assayed for signaling microdomains in T-cell membranes by using single-molecule microscopy (Douglass and Vale, 2005). The diffusion of GFP-labeled raft-like proteins, Lck and LAT, and non-raft-like associated proteins, CD2 and CD45, were analyzed (Douglass and Vale, 2005). Multiple modes of diffusion were observed for individual molecules, with abrupt changes between mobile and immobile states, but a correlation with the raft-association was not detected. In the activated cells, more discrete microdomains (with co-clustering of CD2, LAT and Lck) were formed that were not maintained by actin or lipid raft interactions, as shown by actin-disrupting drugs and mutants that cannot associate to rafts. These domains limit the free diffusion of molecules and exclude or include (and trap) them depending on inter-protein interactions, where phosphorylated LAT plays a pivotal role.

Plasma membrane sheets (introduced in Section 1.4.1.1) have been instrumental tools to study the protein organization as they make the inner membrane leaflet molecules readily accessible. To study the protein organization mast cells membranes were ripped with coated EM grids or coverslips and imaged by atomic force microscopy (AFM) and transmission EM and (Frankel et al., 2006; Wilson et al., 2004). EM imaging uncovered dark patches that exist on the membrane for both resting and activated cells, but become more prominent upon activation by receptor clustering. The patches were formed by enhanced labeling with EM stains such as $\mathrm{OsO}_{4}$, indicating high levels of double-bond containing lipids such as unsaturated lipids, sphingomyelin or cholesterol (Wilson et al., 2004). Immunogold labeling revealed that different signaling proteins known to associate with DRMs were enriched in distinctive patches (Wilson et al., 2004). In AFM topography images irregular raised (thicker) domains that include different signaling components 
were found (Frankel et al., 2006). These domains were cholesterol-dependent and were linked to each other through a fibrous meshwork.

A similar EM study in ripped T-cell membranes targeted a broader range of proteins, by labeling subsets of proteins through biotinylation of their carboxyl or sulfhydryl groups on the cytoplasmic side and coupling to streptavidin-gold (Lillemeier et al., 2006). Like in mast cells the gold-labeled proteins were found to localize in dark patches on the membrane in both resting and activated Tcells. Raft and non-raft markers were both found to be in these patches but clustered in distinct regions within them. These patches that occupied $20-50 \%$ of the membrane were termed "protein islands", which were separated and surrounded by a "sea" of protein-free membrane. Actin and cholesterol were also found to be associated to the protein islands.

In another study the lateral diffusion coefficients of GFP tagged proteins of different topologies (bitopic, polytopic, GPI-anchored and palmitoylated proteins) were measured by FRAP in COS-7 cells (Frick et al., 2007). The observed slow diffusion of proteins in cell membrane was mediated by protein density, and not by cortical cytoskeleton.

A systematic analysis, partly addressing this issue, has recently been performed in yeast cells (Spira et al., 2012). Distributions of $>40$ proteins were sequentially analyzed, through fusions to fluorescent proteins and high-resolution imaging. In each case a heterogeneous distribution was observed, with various type of domains ranging from patches to continuous networks (Spira et al., 2012).

Although these studies summarized above employed different techniques and experimental systems, they all point at the presence of protein-rich domains or assemblies in the plasma membrane. However, the extent or importance of these domains has not been thoroughly investigated.

\subsubsection{Electrostatic Interactions}

The inner leaflet of the plasma membrane contains a high concentration of negatively charged lipids, creating a negative surface potential (van Meer et al., 2008). This makes the membrane organization quite open to electrostatic interactions. For example, the importance of ionic interactions in membrane organization was demonstrated through reversible redistribution of a 
structurally diverse set of proteins in response to changes in the concentrations of divalent cations, especially $\mathrm{Ca}^{2+}$ (Zilly et al., 2011).

An important player in the membrane is the multianionic PIP2 that is known to interact with cationic residues in proteins. PIP2 can bind to many actin-regulating proteins and is involved in stabilizing the interactions between the plasma membrane and the actin cytoskeleton (Liu and Fletcher, 2006; Niggli et al., 1995; Sakisaka et al., 1997). PIP2 is also reported to affect the clustering of certain proteins like syntaxin 1 together with homophilic protein-protein interactions and a complex array of interactions with other proteins, cholesterol and $\mathrm{Ca}^{2+}$, leading to formation of the recruitment sites for vesicle exocytosis (Honigmann et al., 2013b; Lang et al., 2001; Murray and Tamm, 2011; Sieber et al., 2007). PIP2 itself was also demonstrated to be concentrated in domains through local synthesis, interactions with proteins and electrostatic sequestration (Baker, 1952; Honigmann et al., 2013b; McLaughlin et al., 2002; Wang and Richards, 2012).

\subsection{Technical Perspective: High-Resolution Microscopy}

A technical challenge in imaging of domains is to obtain high enough resolution to distinguish the labeled entities from each other. Although electron microscopy can provide higher resolution imaging, it also introduces difficulties in labeling as well as labor-intensive and artifactual sample preparation procedures. On the other hand, optical microscopy provides a direct way for visualization of domains and enables high-density labeling with small probes. Yet, due to diffraction limit of light, the resolution of optical microscopy is limited. Based on the theory of image formation, Ernst Abbe described the physical limit of resolution in optical systems as (Abbe, 1873):

$$
d=\text { Abbe Resolution }_{x, y}=\frac{\lambda}{2 n \sin \alpha}
$$

\section{Equation 1. Abbe's resolution limit}

$\lambda$ is the wavelength of light. $n$ is the refractive index of the medium between the lens and the coverslip (usually air, water, glycerol, or oil) and $\alpha$ is the half angle over which the objective can gather light from the specimen (i.e. half of the angular aperture of the objective).

According to Equation 1, conventional microscopy cannot reach a resolution below $\sim 200 \mathrm{~nm}$. However, this limit is derived from some assumptions, such as the standard design of light collection by the objective and use of uniform excitation light. It also relies on single photon fluorescence with linear absorption and emission properties. Super-resolution microscopy is a collective term for light microscopy techniques that offer resolution beyond the diffraction limit. 
To overcome the diffraction limit, super-resolution techniques emerging in the last two decades have circumvented these assumptions in different ways (Hell, 2007). One way is to modulate the point spread function by exploiting the stimulated emission depletion phenomenon.

The theoretical principles of stimulated emission depletion (STED) microscopy were first introduced in 1994 (Hell and Wichmann, 1994). If an electron in excited state encounters a photon at an energy level similar to the difference between the electron's energy and the ground energy, the photon will stimulate the electron to fall into the ground energy state. This is realized through emission of a photon with the same wavelength as the incoming one. The excited molecule is therefore not allowed to undergo spontaneous emission of a fluorescence photon, but is subject to stimulated emission depletion and will emit a photon at the wavelength of the light used for the STED effect. These more red-shifted photons can be separated from the fluorescence emission by use of filters. Fluorescent dyes can thus be "de-excited" by additional irradiation with the red-shifted stimulated emission depletion beam.

The STED microscope functions like an improved confocal laser-scanning microscope, in which a conventional excitation beam is used to turn on the fluorophores in a diffraction-limited spot (Figure 1-5A). The additional feature is the introduction of a second (STED) beam that is modulated by a phase plate into a doughnut-like shape. By alignment of the two beams the emission of fluorescence is only allowed from the center of the excited spot where the depletion beam has zero intensity, so a much smaller focal spot can be obtained (Hell and Wichmann, 1994; Klar et al., 2000) resulting in images with substantially higher resolution (Figure 1-5B). The image is generated through scanning of the chosen area. The obtained improvement in resolution is theoretically not limited by a physical barrier (Westphal and Hell, 2005). In principle if the intensity of the de-excitation beam is high enough, the focal spot can be decreased to molecular size (Hell, 2003), since it is defined to be independent of diffraction (Equation 2). STED microscopy has been used to uncover the clustered distributions of exo and endocytotic proteins in the plasma membrane (Hoopmann et al., 2010; Opazo et al., 2010; Sieber et al., 2007).

$$
d_{x, y} \approx \frac{\lambda}{2 \sin \alpha \sqrt{1+\frac{I}{I_{s a t}}}}
$$

Equation 2. Lateral resolution in STED microscopy

$\lambda$ and $\alpha$ denote the wavelength and aperture angle of the lens respectively. I is the maximal focal intensity applied for STED and $I_{\text {sat }}$ is a characteristic value at which the fluorescence excitation is reduced to half. Here it could be seen that when $\mathrm{I} / \mathrm{I}_{\mathrm{sat}}$ is increased toward infinity, lateral resolution $\left(\mathrm{d}_{\mathrm{x}, \mathrm{y}}\right)$ approximates to zero; hence resolution is now independent of diffraction (Westphal and Hell, 2005). 
There are also other methods for obtaining super-resolution in optical microscopy. Structured illumination microscopy (SIM) is a wide-field technique that operates through insertion of a periodic diffraction grating of known pattern in the illumination beam path (Heintzmann and Cremer, 1999). The projection of the pattered illumination onto the sample causes the formation of coarse interference patterns, which can be used to back-calculate high-resolution spatial information. Alternatively, localization microscopy techniques such as photoactivated localization microscopy (PALM), stochastic optical reconstruction microscopy (STORM) and fluorescence photoactivation localization microscopy (FPALM) can also provide nanoscale resolution (Betzig et al., 2006; Hess et al., 2006; Rust et al., 2006). These wide-field techniques employ repeated singlemolecule switching cycles to find the localization of single molecules with high precision. The resolution, in this case, is independent of the wavelength and is dependent on the density and accuracy of the localization. In principle, the more photons detected from the molecule, the better is the localization precision. Localization microscopy techniques are recently finding increasing uses in membrane domain studies (Owen et al., 2010; Sengupta et al., 2011).

A

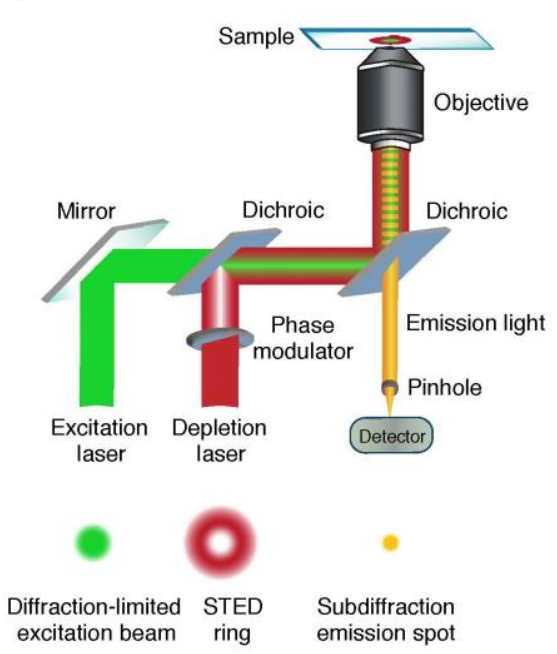

B
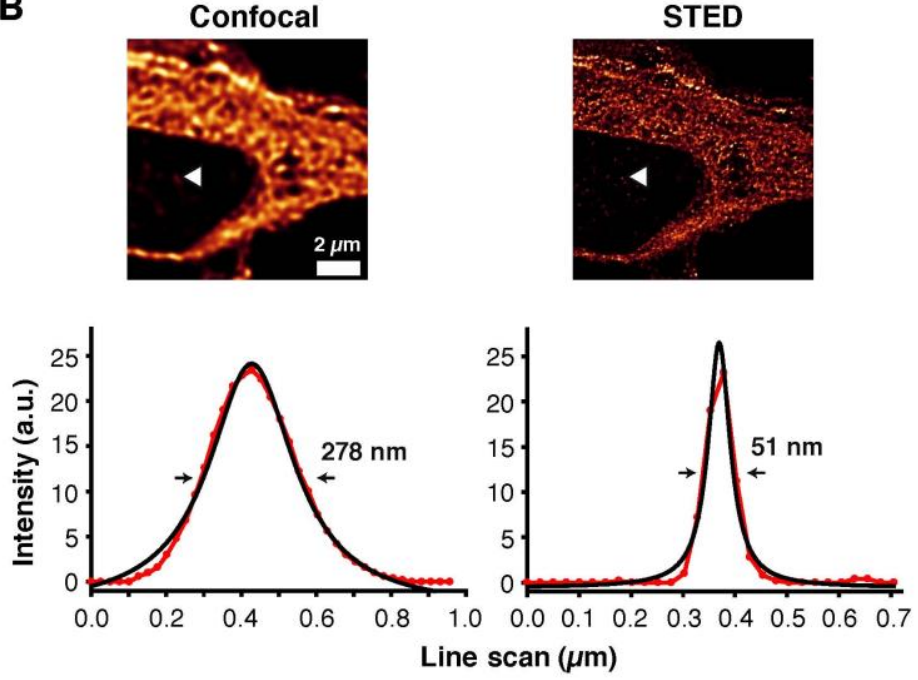

\section{Figure 1-5 STED microscopy}

A. STED imaging. A toroid-shaped depletion beam (red, adjusted through a phase modulator plate) is superimposed on the excitation beam (green), resulting in stimulated emission depletion of all fluorophores except those located in the zero-intensity center of the depletion beam. This combination of STED optics with the confocal elements (such as the detection pinhole or beam scanner) yields an effective fluorescent emission of sub-diffraction size (orange). B. Examples of confocal and STED images of a neuronal cell body immunostained for calnexin, processed by deconvolution. The graphs show line scans (red) through the protein cluster indicated by the white arrowheads. Signal intensities are shown as arbitrary fluorescence units (a.u.). The full-width-half-maximum obtained from Lorentzian fits (black) corresponds to $278 \mathrm{~nm}$ for the confocal and $51 \mathrm{~nm}$ for the STED images. 


\subsection{The Scope of the Thesis}

In the last two decades, it became apparent that the plasma membrane is composed of lipid or protein domains, although many times the particular domains that are observed in different systems are of different characteristics and scales (Maxfield, 2002; Mukherjee and Maxfield, 2004). For protein domains (clusters), two different aspects need to be taken into account: the formation of the domains and the distribution of the domains in the membrane.

The first aspect, cluster formation, is dependent on the nature of the specific proteins. The second aspect, the distribution of the clusters, has been studied less intensively. At first glance, the domains of abundant proteins seem to be distributed randomly across the membrane, for example for syntaxin 1 (Lang et al., 2001; Leydig, 1857; Rickman et al., 2010), syntaxin 4 (Sieber et al., 2006), SNAP25 (Bar-On et al., 2012; Halemani et al., 2010), synaptotagmin 1 (Willig et al., 2006), synaptophysin (Hoopmann et al., 2010), synaptobrevin 2 (Rickman et al., 2010) or amyloid precursor protein (APP, Schreiber et al., 2012). However, when inspected more carefully, a more complex distribution pattern can be seen at least for abundant proteins. The domains do not spread evenly on the entire membrane: they actually gather in small, irregularly shaped areas with a breadth of up to a few hundred nanometers that are separated from each other by narrow regions lacking the protein (see Sieber et al., 2007; Willig et al., 2006 and the examples produced in our experimental conditions in Figure 1-6). The observation of such patterns for several proteins, which are structurally and functionally different, suggests that a common localization mechanism might affect them all. Such a mechanism should be a basic feature of the membrane, a general mechanism, rather than a specialized interaction that affects individual protein species. Since studies of protein patterning are usually performed for a limited number of individual protein species, it is possible that a basic, general organization is overlooked.
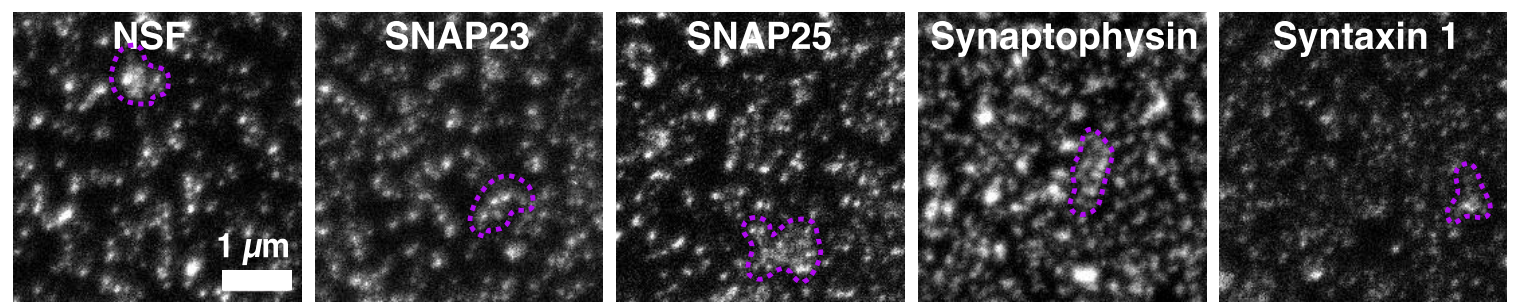

Figure 1-6 Domains of specific proteins.

Representative STED images showing distributions of specific proteins in the membrane of PC12 cells. Membrane fusion related proteins $\mathrm{N}$-ethylmaleimide-sensitive factor (NSF), soluble NSF attachment protein receptors (SNARE) SNAP23, SNAP 25, syntaxin 1, and synaptophysin were immunostained on plasma membrane sheets from PC12 cells. Note the "patterned", not fully homogeneous, distribution of domains. For each image, one example domain has been outlined in purple as a guide to the eye. 
Many general mechanisms of protein localization have been proposed in the past, including the formation of membrane rafts enriched in cholesterol, sphingolipids and specific proteins (Lingwood and Simons, 2010; Pike, 2006), the segregation of specific proteins into areas fenced by actin (Kusumi et al., 2011; Ritchie et al., 2003), the formation of protein islands in a "sea" of protein-poor lipid membranes (Lillemeier et al., 2006), and finally the self-organization of different protein species in individual but overlapping domains, at least in yeast cells (Spira et al., 2012). However, the extent of these mechanisms and their interplay for general protein patterning is still not clear.

In this project I aimed to obtain a better understanding of the mechanisms behind the patterning of protein and protein domains by studying the organization of all proteins through metabolic labeling and super-resolution imaging. The questions I try to answer are as follows:

1. When all proteins are considered, how does the membrane patterning look?

2. What are the factors involved in such patterning? What are the relative contributions of previously suggested membrane organization mechanisms?

3. How does this pattern affect the organization of individual protein species? 


\section{MATERIALS AND METHODS}

\subsection{General Chemicals and Manufacturers}

Laboratory equipment and common or specific chemicals used in this work were either purchased from the following companies or provided by the groups in following institutions (Table 2-1). For the rest of the text, when the companies are specified for individual cases, only the short company name will be given. Please refer to Table 2-1 for more information.

Table 2-1 Company/ institution information

\begin{tabular}{|c|c|c|}
\hline Company Name & City/State & Country \\
\hline Abberior GmbH & Göttingen & Germany \\
\hline Abcam & Cambridge & UK \\
\hline Active Motif & La Hulpe & Belgium \\
\hline AppliChem GmH & Darmstadt & Germany \\
\hline ATTO-TEC GmbH & Siegen & Germany \\
\hline Bandelin Electronic GmbH & Berlin-Lichterfelde & Germany \\
\hline $\begin{array}{l}\text { BD Biosciences (Becton Dickinson } \\
\text { and Company) }\end{array}$ & Franklin Lakes, NJ & USA \\
\hline Calbiochem & San Diego, CA & USA \\
\hline Cameca/ Ametek & Gennevilliers & France \\
\hline Cell Signaling & Beverly, MA & USA \\
\hline Clontech & Saint-Germain-en-Laye & France \\
\hline Dianova & Hamburg & Germany \\
\hline Eppendorf & Hamburg & Germany \\
\hline Fujifilm & Tokyo & Japan \\
\hline GE Healthcare & Little Chalfont & UK \\
\hline Gerhard Menzel GmbH & Braunschweig & Germany \\
\hline Gibco & Paisley & UK \\
\hline Invitrogen & Carlsbad, CA & USA \\
\hline Leica Microsystems GmbH & Mannheim & Germany \\
\hline $\begin{array}{l}\text { Leibniz Institute for Baltic Sea } \\
\text { Research }\end{array}$ & Rostock & Germany \\
\hline Life Technologies & Carlsbad, CA & USA \\
\hline LIMES Institute & Bonn & Germany \\
\hline London Resin Company Ltd & London & UK \\
\hline Lonza GmbH & Cologne & Germany \\
\hline $\begin{array}{l}\text { Max-Planck-Institute for } \\
\text { Biophysical Chemistry (MPI-BPC) }\end{array}$ & Göttingen & Germany \\
\hline $\begin{array}{l}\text { Max-Planck-Institute for } \\
\text { Experimental Medicine (MPI-EM) }\end{array}$ & Göttingen & Germany \\
\hline Merck Millipore & Darmstadt & Germany \\
\hline Molecular Probes & Eugene, OR & USA \\
\hline
\end{tabular}




\begin{tabular}{|lll|}
\hline Newport Spectra-Physics GmbH & Darmstadt & Germany \\
\hline Norvus Biologicals & Cambridge & UK \\
\hline Olympus Corporation & Tokyo & Japan \\
\hline PAA Laboratories GmbH & Clöbe & Germany \\
\hline Santa Cruz Biotechnology & Dallas, TX & USA \\
\hline Scientific Volume Imaging & Hilversum & The Netherlands \\
\hline Sigma-Aldrich & St. Louis, MO & USA \\
\hline Spectrum Laboratories Inc. & Rancho Dominguez, CA & USA \\
\hline $\begin{array}{l}\text { Sorvall Centrifuges - Thermo Fischer } \\
\text { Scientific Inc. }\end{array}$ & Waltham, MA & USA \\
\hline Synaptic Systems & & Germany \\
\hline The Mathworks Inc. & Göttingen & USA \\
\hline
\end{tabular}

\subsection{Instruments}

Table 2-2 Laboratory Equipment

\begin{tabular}{|ll|}
\hline Instrument & Manufacturer \\
\hline Cenoplus Sonifier & Bandelin \\
\hline IX 71 Epi-fluorescence microscope & Eppendorf \\
\hline TCS SP5 STED microscope & Olympus \\
\hline NanoSIMS 50L & Leica \\
\hline EM UC6 ultramicrotome & Cameca \\
\hline Fluorescence Scanner FLA9000 & Leica \\
\hline
\end{tabular}

Table 2-3 Centrifuges and Rotors

\begin{tabular}{|lll|}
\hline Centrifuge & Rotor/Type & Manufacturer \\
\hline Refrigerated table-top centrifuge & $5417 \mathrm{R}$ & Eppendorf \\
\hline Refrigerated centrifuge & $5810 \mathrm{R}$ & Eppendorf \\
\hline Discovery 90SE & TH-641 & Sorvall \\
\hline
\end{tabular}

\subsection{Buffers / Solutions}

Phosphate-buffered saline (PBS): $137 \mathrm{mM} \mathrm{NaCl}, 2.7 \mathrm{mM} \mathrm{KCl}, 10 \mathrm{mM} \mathrm{Na}{ }_{2} \mathrm{HPO}_{4}, 2 \mathrm{mM} \mathrm{KH}_{2} \mathrm{PO}_{4}, \mathrm{pH}$ 7.4 .

Paraformaldehyde (PFA): 4\% PFA (Merck) in PBS.

Preparation: $8 \mathrm{~g}$ PFA was dissolved in $180 \mathrm{ml}$ water through mixing and occasional heating. To increase solubility, $200 \mu \mathrm{l} 10 \mathrm{M} \mathrm{NaOH}$ was added to the mixture. Later, pH was brought back to 7.4 by addition of $\mathrm{HCl}$ and $20 \mathrm{ml}$ 10X PBS, $\mathrm{pH}$ 7.4. The solution was divided into aliquots and frozen to be thawed freshly on the day of the experiment.

PFA + Glutaraldehyde: 4\% PFA and 0.2\% glutaraldehyde (AppliChem) in PBS. 
Sonication buffer: $120 \mathrm{mM}$ potassium glutamate, $20 \mathrm{mM}$ potassium acetate, $10 \mathrm{mM}$ EGTA, $4 \mathrm{mM}$ $\mathrm{MgCl}_{2}, 2 \mathrm{mM}$ ATP (Sigma), 0.5 mM DTT, 20 mM HEPES-KOH, pH 7.2, as described in Lang, 2008).

K-Glu Buffer: $120 \mathrm{mM}$ potassium glutamate, $20 \mathrm{mM}$ potassium acetate, $10 \mathrm{mM}$ EGTA, $4 \mathrm{mM}$ $\mathrm{MgCl}_{2}, 20$ mM HEPES-KOH, pH 7.2.

Click reaction buffers: The buffers for click reaction were purchased from Molecular Probes (Click-iT ${ }^{\circledR}$ Cell Reaction Buffer Kit).

Ringer buffer: $124 \mathrm{mM} \mathrm{NaCl}, 5 \mathrm{mM} \mathrm{KCl}, 2 \mathrm{mM} \mathrm{CaCl}, 1 \mathrm{mM} \mathrm{MgCl}$, $30 \mathrm{mM}$ D-glucose and $25 \mathrm{mM}$ HEPES, pH 7.4.

Dialysis buffer: $8 \mathrm{~g} \mathrm{NaCl}, 0.4 \mathrm{~g} \mathrm{KCl}, 0.35 \mathrm{~g} \mathrm{NaHCO}_{3}, 1 \mathrm{~g}$ glucose, $\mathrm{pH} 7.4$, as described before (Halpern et al., 1974).

Sample buffer: $50 \mathrm{mM}$ Tris- $\mathrm{HCl}$, $4 \%$ sodium doedecyl sulfate (SDS), $0.01 \%$ Serva Blue $\mathrm{G}, 12 \%$ glycerol, pH 6.8.

Homogenization buffer: $250 \mathrm{mM}$ sucrose, 3mM imidazole, pH 7.4 (with $\mathrm{HCl}$ ). Prepared fresh and kept cold (Barysch et al., 2010).

Mowiol: $2.4 \mathrm{~g}$ Mowiol 4-88 (Merck), $6 \mathrm{~g}$ glycerol, $6 \mathrm{ml}$ distilled water and $12 \mathrm{ml} 0.2 \mathrm{M}$ Tris buffer were mixed and the was adjusted to $\mathrm{pH} 8.5$ with $\mathrm{HCl}$. The mixture was stirred up to 5 days with occasional heating (up to $50^{\circ} \mathrm{C}$ ). The solution was divided into aliquots (avoiding the remaining non-dissolved crystals) and snap-frozen to be thaw freshly on the day of the experiment.

\subsection{Cell Culture}

Thin coverslips (<100 $\mu \mathrm{m}$, Gerhard Menzel) of $25 \mathrm{~mm}$ diameter were sequentially washed with 1 $\mathrm{M} \mathrm{NaOH}, 1 \mathrm{M} \mathrm{HCl}$, and 100\% ethanol. They were flamed and coated with $100 \mu \mathrm{g} / \mathrm{ml}$ poly-L-lysine (PLL, Sigma) for $1 \mathrm{~h}$ at RT. PC12 cells (Pheochromocytoma cells, Heumann et al., 1983) were cultured on PLL-coated coverslips as described before (Lang et al., 2001), in Dulbeccos's modified Eagle's medium (DMEM with $4.5 \mathrm{~g} / \mathrm{L}$ glucose, from Lonza) with 100 units/ml penicillin and streptomycin (Lonza), $4 \mathrm{mM}$ glutamine (Lonza), 5\% fetal calf serum (FCS, from PAA) and 10\% horse serum (Merck) with $5-10 \% \mathrm{CO}_{2}$ and $90 \%$ humidity. For passaging, cells were harvested with trypsin/EDTA (Lonza) treatment.

To label all proteins indiscriminately metabolic labeling of proteins was performed. For that, a methionine analogue, L-azidohomoalanine (Click-iT ${ }^{\circledR}$ AHA from Molecular Probes), was used in absence of natural methionine. Therefore, cells were grown in methionine-free medium (methionine-free variants of DMEM or RPMI media). Since serum also contains amino acids, fetal calf serum (FCS) and horse serum were dialyzed to remove the small amino acids. Dialysis was 
done three times for $>8 \mathrm{~h}$ each at $4^{\circ} \mathrm{C}$ in dialysis buffer using a dialysis membrane (Spectrum) with cut-off of $3500 \mathrm{Da}$, followed by filter-sterilization.

AHA-incubation medium for PC12 cells: DMEM (high glucose, no glutamine, no methionine, no cysteine; from Gibco) complemented with $4 \mathrm{mM}$ glutamine, $10 \%$ dialyzed horse serum, $5 \%$ dialyzed fetal calf serum, 100 units/ml penicillin-streptomycin, complemented with $50 \mu \mathrm{M}$ AHA and $400 \mu \mathrm{M}$ L-cysteine (Sigma). In specified cases, $50 \mu \mathrm{M}$ homopropargylglycine (HPG) was used as a methionine analogue instead of AHA.

COS-7 cells (green monkey kidney cells, Invitrogen) were cultured as described before (Barysch et al., 2009) in the same medium as for PC12 cells but with $10 \%$ FCS and no horse serum. For AHA incubation they were also grown for 3 days with AHA as above however with $10 \%$ dialyzed FCS and no horse serum). Alternatively, they were cultured in RPMI medium without methionine (Gibco) instead of methionine and cysteine-free DMEM. All further experiments with COS-7 cells were performed as for PC12 cells.

\subsection{Obtaining the Membrane Sheets}

Membrane sheets were generated by exposing to a brief ultrasound pulse cells in $150 \mathrm{ml}$ ice-cold sonication buffer using a Sonoplus sonifier as shown in Figure 2-1 (Lang, 2008). The distance of the sonication tip and the power of the pulse are experimentally determined for each cell line depending on the adherence of the cells. COS-7 cells were observed to be more adherent than PC12 cells. Therefore, COS-7 cells were sonicated at $75 \%$ of the full power and at a distance of $\sim 0.8 \mathrm{~cm}$, while PC12 cells were sonicated at $60 \%$ and at a distance of $1 \mathrm{~cm}$. The sheets were then washed with K-Glu buffer. Membrane protein organization in membrane sheets is unchanged compared to intact cells, as documented by unaltered membrane protein diffusion (Sieber et al., 2007) and by similar membrane protein patterning (Schreiber et al., 2012). Membrane sheet treatments were performed with the concentrations of reagents and incubation times indicated in the figure legends. All incubations were done at $37^{\circ} \mathrm{C}$, unless otherwise stated. For each experiment, control samples, which were incubated for the same time intervals with buffer alone or buffer with the solvent used for drug applications, typically dimethyl sulfoxide (DMSO), were prepared. 


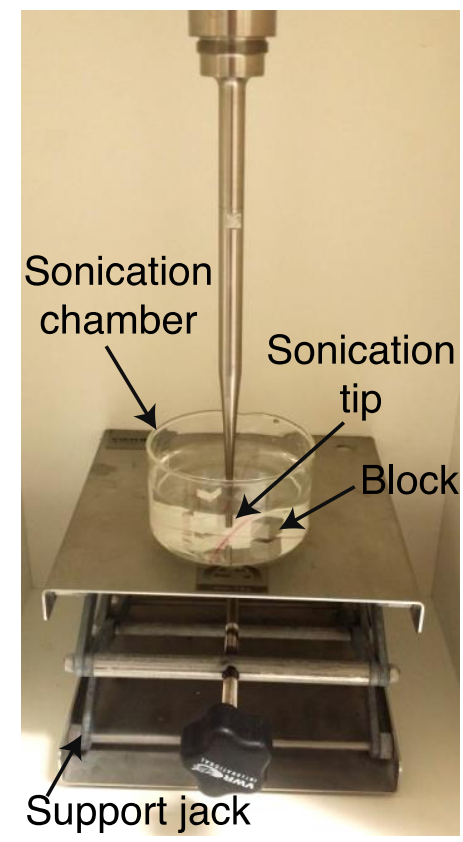

Figure 2-1 The sonication setup.

The coverslip with cells facing up is placed in the center of the sonication chamber filled with ice-cold sonication buffer. The tip of the sonicator is centered in the chamber at a distance of $\sim 0.8-1 \mathrm{~cm}$ from the coverslip. Two $1 \mathrm{~cm}$ high metal blocks are put at the sides of the coverslip in the chamber to be used as reference and also to keep the coverslip in place. A 1-second ultrasound pulse is applied at $65-75 \%$ of the full power

After sonication, PC12 and COS-7 membrane sheets were fixed with 4\% PFA for 10 minutes on ice and another 30 minutes at room temperature (RT) or with 4\% PFA + 0.2\% glutaraldehyde at RT, where indicated. They were then quenched with $100 \mathrm{mM} \mathrm{NH}_{4} \mathrm{Cl}$ for 25 minutes. Unless otherwise indicated in figure legends, click reactions were performed after fixation and quenching, as described below.

\subsection{Click Reactions}

All the clickable chemicals used in the experiments are listed in Table 2-4. Incorporated AHA (or other azide-containing reagents) was coupled to $2 \mu \mathrm{M}$ Atto647N-alkyne (or $5 \mu \mathrm{M}$ Chromeo494alkyne), using the Click-iT ${ }^{\circledR}$ Cell Reaction Buffer Kit (Molecular Probes) according to manufacturer's protocol, by mixing the components following the order and concentrations given in Table 2-5. The table shows a typical reaction mixture for a $250 \mu$ reaction. These volumes are scaled up or down appropriately for the number of coverslips (100 $\mu$ ldrop of reaction mixture per $25 \mathrm{~mm}$ coverslip, incubation performed on parafilm). After addition of each component the tube was mixed vigorously and the reaction mixture is used freshly (in less than 15 minutes). The reaction is let to run for 30 minutes at RT. To label HPG or alkyne containing reagents the same reaction was prepared with azide-modified dyes. To block or remove unspecific binding to the 
membranes, the preparations were washed for 15 minutes with PBS containing $5 \%$ bovine serum albumin (BSA) and 5\% peptone, and briefly with PBS containing 3\% BSA, both before and after the reaction.

Table 2-4 Click reagents

\begin{tabular}{|c|c|c|c|}
\hline Click reagents & Company & Final concentration & $\begin{array}{l}\text { Incubation } \\
\text { duration }\end{array}$ \\
\hline L-azidohomoalanine (AHA) & Molecular Probes & $50 \mu \mathrm{M}$ & $72 \mathrm{~h}$ \\
\hline Homopropargylglycine (HPG) & Molecular Probes & $50 \mu \mathrm{M}$ & $72 \mathrm{~h}$ \\
\hline Tetraacetylfucose alkyne & Molecular Probes & $50 \mu \mathrm{M}$ & $72 \mathrm{~h}$ \\
\hline $\begin{array}{l}\text { Tetraacetylated N-azidoacetyl- } \\
\text { D-mannosamine (ManNAz) }\end{array}$ & Molecular Probes & $50 \mu \mathrm{M}$ & $72 \mathrm{~h}$ \\
\hline $\begin{array}{l}\text { Tetraacetylated } \mathrm{N} \text { - } \\
\text { azidoacetylgalactosamine } \\
\text { (GalNAz) }\end{array}$ & Molecular Probes & $50 \mu \mathrm{M}$ & $72 \mathrm{~h}$ \\
\hline $\begin{array}{l}\text { Tetraacetylated } \mathrm{N} \text { - } \\
\text { azidoacetylglucosamine } \\
\text { (GlcNAz) }\end{array}$ & Molecular Probes & $50 \mu \mathrm{M}$ & $72 \mathrm{~h}$ \\
\hline Geranylgeranyl alcohol azide & Molecular Probes & $50 \mu \mathrm{M}$ & $48 \mathrm{~h}$ \\
\hline Farnesyl alcohol azide & Molecular Probes & $50 \mu \mathrm{M}$ & $48 \mathrm{~h}$ \\
\hline Palmitic acid azide & Molecular Probes & $200 \mu \mathrm{M}$ & $24 \mathrm{~h}$ \\
\hline Myristic acid azide & Molecular Probes & $50 \mu \mathrm{M}$ & $24 \mathrm{~h}$ \\
\hline \multicolumn{4}{|l|}{ Fluorescent dyes for click } \\
\hline Atto647N-alkyne & Atto-tec & $2 \mu \mathrm{M}$ & \\
\hline Atto647N-azide & Atto-tec & $2 \mu \mathrm{M}$ & \\
\hline Chromeo494-azide & Active Motif & $5 \mu \mathrm{M}$ & \\
\hline Chromeo494-alkyne & Active Motif & $5 \mu \mathrm{M}$ & \\
\hline
\end{tabular}

Table 2-5 Click reaction mixture for cells

\begin{tabular}{|ll|}
\hline Double-distilled water & Volume \\
\hline $\begin{array}{l}\text { Click reaction buffer } \\
\text { (Component A) }\end{array}$ & $22 \mu \mathrm{ll}$ \\
\hline $\begin{array}{l}\mathbf{1 0 0} \boldsymbol{\mu M} \text { CuSO } \\
\text { (Component B) }\end{array}$ & $5 \mu \mathrm{l}$ \\
\hline $\begin{array}{l}\text { Click reaction buffer additive } \\
\text { (Component C) }\end{array}$ & $25 \mu \mathrm{l}$ \\
\hline Clickable dye with complementary chemistry & $2 \mu \mathrm{l}$ (diluted to get the desired final concentration) \\
\hline Total & $250 \mu \mathrm{l}$ \\
\hline
\end{tabular}

\subsubsection{Click Labeling of Protein Modifiers}

To label various modified subsets of proteins, in parallel to 3-day AHA or HPG incubation for general protein labeling, PC12 cells were also incubated with one of the following metabolic 
labeling reagents listed in Table 2-4. The incubations were done with the given concentrations and for given durations in AHA-incubation medium, either in presence of AHA or HPG (chosen based on complementary functionality to allow clicking to different fluorophores). Two click reactions were performed sequentially on membrane sheets obtained from these cells (separated by thorough washes), the first to label the proteins with Chromeo494, and the second to label the protein modifiers with Atto647N.

\subsection{Plastic Embedding}

As an alternative to sonication, whole cells were fixed and labeled by click chemistry as above. They were then embedded in 2,4,6-Tris[bis(methoxymethyl)amino]-1,3,5-triazine (also known as melamin), as described in the past (Hoopmann et al., 2010); this was followed by thin-section processing using an EM UC6 ultramicrotome, again as previously described (Hoopmann et al., 2010). Sections of $10-20 \mathrm{~nm}$ thickness were used for STED imaging.

\subsection{Treatments / Perturbations}

\subsubsection{Trypsinization of Membrane Sheets}

For a control of protein labeling specificity, after sonication PC12 membranes were washed with sonication buffer containing 3\% BSA and subject to click reaction with Atto647N-alkyne without fixation. Following several washes with sonication buffer containing 3\% BSA, unfixed PC12 membrane sheets were treated with $1 \mathrm{mg} / \mathrm{ml}$ trypsin (from bovine pancreas, Sigma) or with 1 $\mathrm{mg} / \mathrm{ml}$ trypsin and $2 \mathrm{mg} / \mathrm{ml}$ trypsin inhibitor (Sigma). STED images were taken just before the addition of trypsin or trypsin + inhibitor and at 15 and 35 minutes after the addition.

\subsubsection{Ionic Alterations}

After sonication, membrane sheets were incubated for 20 minutes in high-salt buffer (sonication buffer containing $500 \mathrm{mM} \mathrm{KCl}$ ), low ionic strength buffer (320 mM sucrose, $5 \mathrm{mM} \mathrm{HEPES-KOH}, \mathrm{pH}$ 7.2) or high-calcium buffer (sonication buffer containing $1 \mathrm{mM} \mathrm{Ca}{ }^{2+}$ ). Alternatively, cells were incubated with $1 \mu \mathrm{M}$ ionomycin (Sigma) for 5 minutes in HEPES-buffered DMEM (Gibco), in the presence of $5 \mathrm{mM} \mathrm{CaCl}_{2}$, with or without $10 \mathrm{mM} \mathrm{EGTA}$. Membrane sheets were obtained from the treated cells after 3 brief washes with HEPES-buffered DMEM. 


\subsubsection{Cytoskeleton Disruptions}

Before sonication, cells were incubated for $1 \mathrm{~h}$ in the AHA-incubation medium described above, containing $10 \mu \mathrm{M}$ nocodazole (Sigma), $10 \mu \mathrm{M}$ colchicine (Sigma), $1 \mu \mathrm{M}$ latrunculin A (Calbiochem), $10 \mu \mathrm{M}$ cytochalasin D (Sigma) or $0.1 \%$ DMSO (as solvent control). They were then washed and subjected to sonication, followed by $4 \%$ PFA fixation.

\subsubsection{Lipid Perturbations}

For sphingomyelin removal, membranes were incubated with 20 units $/ \mathrm{ml}$ sphingomyelinase (Sigma) for 15 minutes at $37^{\circ} \mathrm{C}$, washed and fixed. The following phospholipases (PL; from Sigma) were similarly applied in the given final concentrations: 2 units/ml of PLA2 from bovine pancreas, 2 units $/ \mathrm{ml}$ of PLC from Clostridium perfringens, 1 unit/ml of phosphatidylinositol-specific PLC (PIPLC) from Bacillus cereus, and 2 units/ml of PLD from Streptomyces sp. For cholesterol depletion, membranes were incubated for 5 minutes with $5 \mathrm{mM} \mathrm{MBCD}$ (Sigma), dissolved in sonication buffer at $37^{\circ} \mathrm{C}$. This step was followed by 3 washes with buffer, and by either a 10-minute incubation in sonication buffer at $37^{\circ} \mathrm{C}$ or a 10 -minute incubation in sonication buffer containing cholesterol complexed with $5 \mathrm{mM} \mathrm{MBCD}$ at 1:8 molar ratio (for membrane repletion with cholesterol), as described previously (Christian et al., 1997). Alternatively, membranes were incubated with 0.2 units $/ \mathrm{ml}$ cholesterol oxidase (Sigma) for 15 minutes, or with $25 \mathrm{mM}$ (2Hydroxypropyl)- $\alpha$-cyclodextrin (Sigma) or (2-Hydroxypropyl)- $\beta$-cyclodextrin (Sigma) for 5 minutes at $37^{\circ} \mathrm{C}$; this was followed by three washes and a 10-minute incubation in sonication buffer (to keep the total incubation time constant across different conditions).

\subsection{Genetic Constructs}

\subsubsection{Plasmids}

All coding sequences used are listed in Table 2-6. The plasmids were generously provided by the indicated colleagues and collaborators. 
Table 2-6 Plasmids

\begin{tabular}{|c|c|c|c|c|}
\hline Coding Sequence & Explanation & Source Vector & Provided by & Reference \\
\hline $\begin{array}{l}\text { Syntaxin1- } \\
\text { pHluorin }\end{array}$ & $\begin{array}{l}\text { Rat Syntaxin } 1 \\
\text { sequence fused to } \\
\text { pHluorin, a pH- } \\
\text { sensitive green } \\
\text { fluorescent protein } \\
\text { (GFP) variant) }\end{array}$ & $\begin{array}{l}\text { pEGFP-N1 } \\
\text { (Clontech) } \\
\text { (EGFP was } \\
\text { replaced with } \\
\text { pHluorin) }\end{array}$ & & $\begin{array}{c}\text { (Hoopmann et al., } \\
\text { 2010) }\end{array}$ \\
\hline YFP-SNAP25 & $\begin{array}{l}\text { Yellow fluorescent } \\
\text { protein (YFP) fused to } \\
\text { rat SNAP25b }\end{array}$ & $\begin{array}{l}\text { pEYFP-C1 } \\
\text { (Clontech) }\end{array}$ & $\begin{array}{c}\text { Thorsten Lang } \\
\text { (LIMES } \\
\text { Institute) }\end{array}$ & (Zilly et al., 2011) \\
\hline $\begin{array}{l}\text { GFP-SNAP25 linker } \\
\text { domain }\end{array}$ & $\begin{array}{l}\text { GFP sequence fused } \\
\text { to a truncated } \\
\text { SNAP25 mutant, } \\
\text { includes the linker } \\
\text { domain for } \\
\text { palmitoylation, but } \\
\text { lacks both SNARE } \\
\text { domains }\end{array}$ & $\begin{array}{c}\text { pEGFP-C1 } \\
\text { (Clontech) } \\
\text { (original EGFP } \\
\text { was exchanged } \\
\text { with monomeric } \\
\text { GFP) }\end{array}$ & $\begin{array}{c}\text { Thorsten Lang } \\
\text { (LIMES } \\
\text { Institute) }\end{array}$ & $\begin{array}{c}\text { (Halemani et al., } \\
\text { 2010) }\end{array}$ \\
\hline GPI-ACP & $\begin{array}{l}\text { GPI-anchor signal } \\
\text { sequence fused to } \\
\text { acyl carrier protein } \\
\text { (ACP) }\end{array}$ & & $\begin{array}{l}\text { Alf Honigmann } \\
\text { (MPI-BPC) }\end{array}$ & (Eggeling et al., 2009) \\
\hline
\end{tabular}

\subsubsection{Small Interfering RNAs (siRNAs)}

Syntaxin 1 and SNAP 25 knockdowns were performed with siRNAs. A combination of 3 siRNAs was employed to knockdown both isoforms of SNAP25, while 5 siRNAs were used to target both syntaxin $1 \mathrm{~A}$ and $1 \mathrm{~B}$, as listed in Table 2-7. Except SNAP25_1, all siRNAs were designed by Ingrid-Cristiana Vreja (University of Göttingen Medical Center). Constructs were purchased from Thermo Scientific Dharmacon ${ }^{\circledR}$ as $2^{\prime}$-deprotected duplexes and resuspended in RNase-free solutions. The GenBank accession numbers for the sequences used are: NM_030991.2 for SNAP-25, NM_053788.2 for Stx1A and NM_012700.2 for Stx1B.

Table 2-7 List of siRNAs used for knockdown

\begin{tabular}{|ll|}
\hline SiRNA & Target Sequence \\
\hline SNAP25_1 (Cahill et al., 2006) & 5'-GTTGGATGAGCAAGGCGAA-3' \\
\hline SNAP25_2 & 5'-GGATGAGCAAGGCGAACAA-3' \\
\hline SNAP25_3 & 5'-TAATATAGGGTTTGTCGAA-3' \\
\hline Syntaxin1A_1 & 5'-CACCAAAGGTCTCGGTAC-3' \\
\hline Syntaxin1A_2 & 5'-TTAAGAAGACAGCGAACA-3' \\
\hline Syntaxin1B_1 & 5'-GGTCCAAGTTGAAAGCGAT-3' \\
\hline Syntaxin1B_2 & 5'-GGAGGTAATGACCGAATAT-3' \\
\hline Syntaxin1A_4 & 5'-GCTAAAGAGCATCGAGCAG-3' \\
\hline
\end{tabular}




\subsubsection{Transfection}

For transfection of plasmid DNA or siRNAs, Lipofectamine 2000 (Invitrogen) was used according to manufacturer's protocol. Briefly, per $25 \mathrm{~mm}$ coverslips, 2-2.5 $\mu \mathrm{g}$ plasmid DNA and 6-7 $\mu \mathrm{l}$ Lipofectamine 2000 were separately diluted in $200 \mu$ Opti-MEM Reduced Serum Medium (Gibco). They were then mixed together and incubated for 20 minutes before addition onto cells.

For siRNA transfection, $100 \mathrm{pmol}$ of each siRNA oligomer (per $25 \mathrm{~mm}$ coverslip) and $5 \mu \mathrm{l}$ Lipofectamine 2000 Reagent were separately diluted in $250 \mu$ Opti-MEM Reduced Serum Medium. They were then mixed and incubated for 20 minutes at room temperature, and applied onto the cells.

Transfections were performed on AHA-incubated PC12 or COS-7 cells during the second day of the AHA incubation, and cells were allowed to express the proteins for 48 hours. 24 hours after transfection, cell media were exchanged.

\subsection{Labeling Protocols}

\subsubsection{Immunostainings}

After click reaction, membrane sheets were immunstained with antibodies against cytoplasmic epitopes according to Table 2-8. An exception was made for flotillin 2 and PIP2 antibodies, which were applied onto sheets for 15 minutes prior to fixation.

Table 2-8 List of antibodies used for immunostainings

\begin{tabular}{|c|c|c|c|c|c|}
\hline $\begin{array}{l}\text { Antibodies } \\
\text { against }\end{array}$ & Company & $\begin{array}{l}\text { Catalogue } \\
\text { number/ } \\
\text { clone }\end{array}$ & Species & Epitope & Incubation \\
\hline Actin (pan) & Norvus & $\begin{array}{l}\text { NB 600-535/ } \\
\text { ACTN05 } \\
\text { (CO4) }\end{array}$ & $\begin{array}{l}\text { Mouse } \\
\text { monoclonal }\end{array}$ & Cytoplasmic & $\begin{array}{l}1: 75 \text { in } 1.5 \% \text { BSA- } \\
\text { PBS, } 1 \mathrm{~h}, \mathrm{RT}\end{array}$ \\
\hline APP & Synaptic Systems & 127002 & $\begin{array}{l}\text { Rabbit } \\
\text { polyclonal }\end{array}$ & Cytoplasmic & $\begin{array}{l}1: 250 \text { in } 1.5 \% \text { BSA- } \\
\text { PBS, } 1 \mathrm{~h}, \mathrm{RT}\end{array}$ \\
\hline $\begin{array}{l}\beta \text {-secretase } \\
\text { (BACE) }\end{array}$ & Santa Cruz & Sc10748 & $\begin{array}{l}\text { Rabbit } \\
\text { polyclonal }\end{array}$ & Cytoplasmic & $\begin{array}{l}1: 100 \text { in } 3 \% \text { BSA-PBS, } \\
1 \mathrm{~h}, \mathrm{RT}\end{array}$ \\
\hline Bassoon & Synaptic Systems & 141002 & $\begin{array}{l}\text { Rabbit } \\
\text { polyclonal }\end{array}$ & Cytoplasmic & $\begin{array}{l}1: 250 \text { in } 1.5 \% \text { BSA- } \\
\text { PBS, } 1 \mathrm{~h}, \mathrm{RT}\end{array}$ \\
\hline$\alpha-\mathrm{N}$-catenin & Cell Signaling & 2131 & $\begin{array}{l}\text { Rabbit } \\
\text { polyclonal }\end{array}$ & Cytoplasmic & $\begin{array}{l}\text { 1:100 in } 1.5 \% \text { BSA- } \\
\text { PBS, } 1 \mathrm{~h}, \mathrm{RT}\end{array}$ \\
\hline
\end{tabular}




\begin{tabular}{|c|c|c|c|c|c|}
\hline Caveolin 1 & $\begin{array}{l}\text { Abcam (provided } \\
\text { by Thorsten Lang, } \\
\text { LIMES Institute) }\end{array}$ & ab2910 & $\begin{array}{l}\text { Rabbit } \\
\text { monoclonal }\end{array}$ & Cytoplasmic & $\begin{array}{l}1: 100 \text { in } 3 \% \text { BSA-PBS, } \\
1 \mathrm{~h}, \mathrm{RT}\end{array}$ \\
\hline $\begin{array}{l}\text { Clathrin heavy } \\
\text { chain }\end{array}$ & BD Biosciences & 10499 & $\begin{array}{l}\text { Mouse } \\
\text { monoclonal }\end{array}$ & Cytoplasmic & $\begin{array}{l}\text { 1:100 in } 1.5 \% \text { BSA- } \\
\text { PBS, } 1 \mathrm{~h}, \mathrm{RT}\end{array}$ \\
\hline Cortactin & Synaptic Systems & 313111 & $\begin{array}{l}\text { Mouse } \\
\text { monoclonal }\end{array}$ & Cytoplasmic & $\begin{array}{l}1: 100 \text { in } 1.5 \% \text { BSA- } \\
\text { PBS, } 1 \mathrm{~h}, \mathrm{RT}\end{array}$ \\
\hline Dynamin $1,2,3$ & BD Biosciences & 610245 & $\begin{array}{l}\text { Mouse } \\
\text { monoclonal }\end{array}$ & Cytoplasmic & $\begin{array}{l}1: 100 \text { in } 1.5 \% \text { BSA- } \\
\text { PBS, } 1 \mathrm{~h}, \mathrm{RT}\end{array}$ \\
\hline $\begin{array}{l}\text { ERM } \\
\text { phospho-ezrin } \\
\text { (Thr567) } \\
\text { /radixin } \\
\text { (Thr564) / } \\
\text { moesin } \\
\text { (Thr558) }\end{array}$ & Cell Signaling & 3149 & $\begin{array}{l}\text { Rabbit } \\
\text { polyclonal }\end{array}$ & Cytoplasmic & $\begin{array}{l}1: 75 \text { in } 1.5 \% \text { BSA- } \\
\text { PBS, } 1 \mathrm{~h}, \mathrm{RT}\end{array}$ \\
\hline GFP & Molecular Probes & $\begin{array}{l}\text { A-11120/ } \\
3 E 6\end{array}$ & $\begin{array}{l}\text { Mouse } \\
\text { monoclonal }\end{array}$ & & $\begin{array}{l}\text { 1:100 in } 1.5 \% \text { BSA- } \\
\text { PBS, } 1 \mathrm{~h}, \mathrm{RT}\end{array}$ \\
\hline Flotillin 2 & $\begin{array}{l}\text { Santa Cruz } \\
\text { (provided by Anja } \\
\text { Schneider, MPI- } \\
\text { EM) }\end{array}$ & & $\begin{array}{l}\text { Mouse } \\
\text { monoclonal }\end{array}$ & Cytoplasmic & $\begin{array}{l}\text { 1:50 in sonication } \\
\text { buffer, } 15 \mathrm{~min}, 4^{\circ} \mathrm{C}\end{array}$ \\
\hline Munc18-1 & Synaptic Systems & 116002 & $\begin{array}{l}\text { Rabbit } \\
\text { polyclonal }\end{array}$ & Cytoplasmic & $\begin{array}{l}1: 100 \text { in } 1.5 \% \text { BSA- } \\
\text { PBS, } 1 \mathrm{~h}, \mathrm{RT}\end{array}$ \\
\hline NSF & Synaptic Systems & 123002 & $\begin{array}{l}\text { Rabbit } \\
\text { polyclonal }\end{array}$ & Cytoplasmic & $\begin{array}{l}1: 250 \text { in } 1.5 \% \text { BSA- } \\
\text { PBS, } 1 \mathrm{~h}, \mathrm{RT}\end{array}$ \\
\hline PIP2 & Abcam & $\begin{array}{l}\text { ab11039/ } \\
\text { 2c11 }\end{array}$ & monoclonal & & $\begin{array}{l}\text { 1:50 in sonication } \\
\text { buffer, } 15 \mathrm{~min}, 4^{\circ} \mathrm{C}\end{array}$ \\
\hline$\alpha$-SNAP & Synaptic Systems & 112111 & $\begin{array}{l}\text { Mouse } \\
\text { monoclonal }\end{array}$ & Cytoplasmic & $\begin{array}{l}\text { 1:100 in } 1.5 \% \text { BSA- } \\
\text { PBS, } 1 \mathrm{~h}, \mathrm{RT}\end{array}$ \\
\hline SNAP23 & Synaptic Systems & 111202 & $\begin{array}{l}\text { Rabbit } \\
\text { polyclonal }\end{array}$ & Cytoplasmic & $\begin{array}{l}1: 100 \text { in } 1.5 \% \text { BSA- } \\
\text { PBS, } 1 \mathrm{~h}, \mathrm{RT}\end{array}$ \\
\hline SNAP25 & Synaptic Systems & $\begin{array}{l}111011 / \\
71.1\end{array}$ & $\begin{array}{l}\text { Mouse } \\
\text { monoclonal }\end{array}$ & Cytoplasmic & $\begin{array}{l}1: 100 \text { in } 1.5 \% \text { BSA- } \\
\text { PBS, } 1 \mathrm{~h}, \mathrm{RT}\end{array}$ \\
\hline Synaptophysin & $\begin{array}{l}\text { Provided by } \\
\text { Reinhard Jahn } \\
\text { (MPI-BPC) }\end{array}$ & G95 and G96 & $\begin{array}{l}\text { Rabbit } \\
\text { polyclonal }\end{array}$ & $\begin{array}{c}\text { Cytoplasmic } \\
\text { and } \\
\text { extracellular }\end{array}$ & $\begin{array}{l}1: 100 \text { in } 1.5 \% \text { BSA- } \\
\text { PBS, } 1 \mathrm{~h}, \mathrm{RT}\end{array}$ \\
\hline Synaptotagmin & Synaptic Systems & $\begin{array}{l}105311 / \\
604.2\end{array}$ & $\begin{array}{l}\text { Mouse } \\
\text { monoclonal }\end{array}$ & Extracellular & $\begin{array}{l}1: 100 \text { in } 1.5 \% \text { BSA- } \\
\text { PBS, } 1 \mathrm{~h}, \mathrm{RT}\end{array}$ \\
\hline Syntaxin 1 & Synaptic Systems & $\begin{array}{l}110001 / \\
78.2(\mathrm{HPC}-1)\end{array}$ & $\begin{array}{l}\text { Mouse } \\
\text { monoclonal }\end{array}$ & Cytoplasmic & $\begin{array}{l}1: 100 \text { in } 1.5 \% \text { BSA- } \\
\text { PBS, } 1 \mathrm{~h}, \mathrm{RT}\end{array}$ \\
\hline $\begin{array}{l}\text { Syntaxin } 1 \\
\text { (Fab fragment } \\
\text { directly } \\
\text { labelled with } \\
\text { Atto647N) }\end{array}$ & Synaptic Systems & 110011 & $\begin{array}{l}\text { Mouse } \\
\text { monoclonal }\end{array}$ & Cytoplasmic & $\begin{array}{l}1: 100 \text { in } 1.5 \% \text { BSA- } \\
\text { PBS, } 1 \mathrm{~h}, \mathrm{RT}\end{array}$ \\
\hline Syntaxin 13 & Synaptic Systems & 110133 & $\begin{array}{l}\text { Rabbit } \\
\text { polyclonal }\end{array}$ & Cytoplasmic & $\begin{array}{l}1: 100 \text { in } 1.5 \% \text { BSA- } \\
\text { PBS, } 1 \mathrm{~h}, \mathrm{RT}\end{array}$ \\
\hline Syntaxin 4 & Synaptic Systems & 110042 & $\begin{array}{l}\text { Rabbit } \\
\text { polyclonal }\end{array}$ & Cytoplasmic & $\begin{array}{l}\text { 1:100 in } 1.5 \% \text { BSA- } \\
\text { PBS, } 1 \mathrm{~h}, \mathrm{RT}\end{array}$ \\
\hline TfnR & $\begin{array}{l}\text { Novex (Life } \\
\text { Technologies) }\end{array}$ & $\begin{array}{l}13-6800 \text { / } \\
\mathrm{H} 68.4\end{array}$ & $\begin{array}{l}\text { Mouse } \\
\text { monoclonal }\end{array}$ & Cytoplasmic & $\begin{array}{l}1: 100 \text { in } 1.5 \% \text { BSA- } \\
\text { PBS, } 1 \mathrm{~h}, \mathrm{RT}\end{array}$ \\
\hline
\end{tabular}




\begin{tabular}{|c|c|c|c|c|c|}
\hline TfnR & Abcam & Ab1086 & $\begin{array}{l}\text { Mouse } \\
\text { monoclonal }\end{array}$ & Extracellular & $\begin{array}{l}1: 100 \text { in } 1.5 \% \text { BSA- } \\
\text { PBS, } 1 \mathrm{~h}, \mathrm{RT}\end{array}$ \\
\hline$\alpha$-Tubulin & Synaptic Systems & 302203 & $\begin{array}{l}\text { Rabbit } \\
\text { polyclonal }\end{array}$ & Cytoplasmic & $\begin{array}{l}1: 200 \text { in } 1.5 \% \text { BSA- } \\
\text { PBS, } 1 \mathrm{~h}, \mathrm{RT}\end{array}$ \\
\hline \multicolumn{6}{|c|}{ Secondary antibodies } \\
\hline $\begin{array}{l}\text { Rabbit IgG } \\
\text { (Atto647N- } \\
\text { coupled) }\end{array}$ & Sigma & 40839 & & & $\begin{array}{l}1: 100 \text { in } 1.5 \% \text { BSA- } \\
\text { PBS, } 1 \mathrm{~h}, \mathrm{RT}\end{array}$ \\
\hline $\begin{array}{l}\text { Mouse IgG } \\
\text { (Atto647N- } \\
\text { coupled) }\end{array}$ & Sigma & 50185 & & & $\begin{array}{l}1: 100 \text { in } 1.5 \% \text { BSA- } \\
\text { PBS, } 1 \mathrm{~h}, \mathrm{RT}\end{array}$ \\
\hline $\begin{array}{l}\text { Mouse IgG } \\
\text { (Star635- } \\
\text { coupled) }\end{array}$ & $\begin{array}{l}\text { Abberior, } \\
\text { Goettingen, } \\
\text { Germany }\end{array}$ & $\begin{array}{l}2-0002-002- \\
0\end{array}$ & & & $\begin{array}{l}1: 75 \text { in } 1.5 \% \text { BSA- } \\
\text { PBS, } 1 \mathrm{~h}, \mathrm{RT}\end{array}$ \\
\hline
\end{tabular}

\subsubsection{Labeling of Whole Cells from the Extracellular Side}

PC12 cells were incubated in Ringer buffer on ice for $1 \mathrm{~h}$ with Atto647N conjugated $\alpha$ bungarotoxin (Molecular Probes). These cells were later sonicated to obtain membrane sheets and were then fixed and labeled by click reactions. Alternatively, whole cell immunostainings were performed as follows. Several primary antibodies with against extracellular epitopes (as listed in Table 2-8) were applied from extracellular side onto whole PC12 cells fixed with 4\% PFA and $0.2 \%$ glutaraldehyde. For the whole cell experiments, fluorescently labeled fixed cells were embedded in plastic resin and sectioned as indicated above under "Plastic Embedding".

\subsubsection{ACP Reaction}

COS-7 cells were transfected with GPI-ACP plasmid (Table 2-6). At the day of the experiment, the cells were incubated with ACP-synthase and Atto647-Coenzyme $A$ in the cell medium for 30 minutes at $37^{\circ} \mathrm{C}$. ACP-synthase reaction specifically conjugates Atto647 onto $A C P$, as previously explained (Eggeling et al., 2009). After labeling and washing, cells were fixed and sonicated as standard.

\subsubsection{Aptamer Labeling}

Atto647N-coupled TfnR aptamer (sequence published in Wilner et al., 2012) was prepared as previously described (Opazo et al., 2012). Fresh before use, it was folded by heating in a thermocycler to $75^{\circ} \mathrm{C}$ (for 3 minutes in PBS containing $5 \mathrm{mM} \mathrm{MgCl}_{2}$ ), followed by cooling to $20^{\circ} \mathrm{C}$ at a rate of $1^{\circ} \mathrm{C} / \mathrm{min}$. $\mathrm{PC} 12$ cells were incubated for 20 minutes on ice with a final dilution of 400 
$\mathrm{nM}$ of the folded aptamer in Ringer buffer containing $1 \mu \mathrm{g} / \mathrm{ml}$ salmon sperm DNA as a blocking reagent. The cells were then washed with Ringer buffer and sonicated. Membrane sheets were labeled with Chromeo494 through click reactions either directly after sonication (unfixed) or after fixation (with 4\% PFA for 20 minutes on ice, and for 30 minutes at RT) and quenching. The unfixed sheets were washed and imaged in K-Glu buffer immediately after preparation, whereas the fixed membranes were embedded in Mowiöl before imaging.

\subsubsection{Synthesis of Cholesterol-PEG-KK114}

To synthesize a cholesterol analogue containing a far-red-fluorescent dye suitable for STEDmicroscopy, a strategy which is known to dramatically improve the partitioning of other lipid analogues dramatically was employed (Honigmann et al., 2013a). Briefly, a long water-soluble PEG linker was placed between the membrane probe and the dye to keep the dye in the water phase. Chol-PEG(3400)-KK114 (structure is shown in Figure 2-2) was prepared from $1 \mu \mathrm{mol}$ CholPEG(3400)-amine (Nanocs Inc, USA) in $0.2 \mathrm{ml}$ of dry N,N-dimethylformamide, $20 \mu \mathrm{L}(0.15 \mathrm{nmol}$ ) of triethylamine and $1 \mu \mathrm{mol}$ of the NHS ester of the fluorescent dye KK114 (Kolmakov et al., 2012). The product was isolated by elution with a chloroform:methanol:water mixture (70:25:3). These steps were kindly performed by Vladimir Belov (MPI-BPC).

\subsubsection{Characterization of Phase Partitioning of Chol-PEG-KK114 in GUVs}

GUVs were prepared by swelling dried lipid films deposited on low melting point agarose (Horger et al., 2009). Briefly, $100 \mu$ l of a pre-heated $1 \%$ agarose solution was spin-coated (3000 rpm) on cleaned coverslips. After spin-coating, the agarose was dried by heating the coverslips to $40^{\circ} \mathrm{C}$ for 30 minutes on a heating plate. Then, $30 \mu \mathrm{l}$ of a $5 \mathrm{~g} / \mathrm{l}$ lipid solution of Dioleoyl phosphatidylcholine (DOPC)/brain sphingomyelin/cholesterol, 2:2:1 (Veatch and Keller, 2005), dissolved in methanol/chloroform (1:1) was spin-coated (3000 rpm) on top of the agarose and residual solvent was removed by applying a vacuum for 20 minutes. Finally, the dried lipid film was hydrated in pure water at $50{ }^{\circ} \mathrm{C}$ for 5 minutes and then slowly cooled to RT. The lipid mixture contained 0.01 mol \% of Chol-PEG-KK114 and DiO. To estimate the partitioning of Chol-PEG-KK114 between the $L_{d}$ and the $L_{0}$ phase GUVs were imaged at the equatorial plane (Figure 2-2) and the distribution of DiO was used to identify phases (bright is $L_{d}$, dark is $L_{0}$ ). $L_{0}$ partitioning was calculated according to $\mathrm{L}_{\mathrm{o}} \%=$ Intensity $\mathrm{LO}_{\mathrm{o}} /$ (Intensity $\mathrm{LO}_{\mathrm{O}}+$ Intensity $\left.\mathrm{Ld}_{\mathrm{d}}\right)$. The characterization procedure was kindly performed by Alf Honigmann (MPI-BPC). 

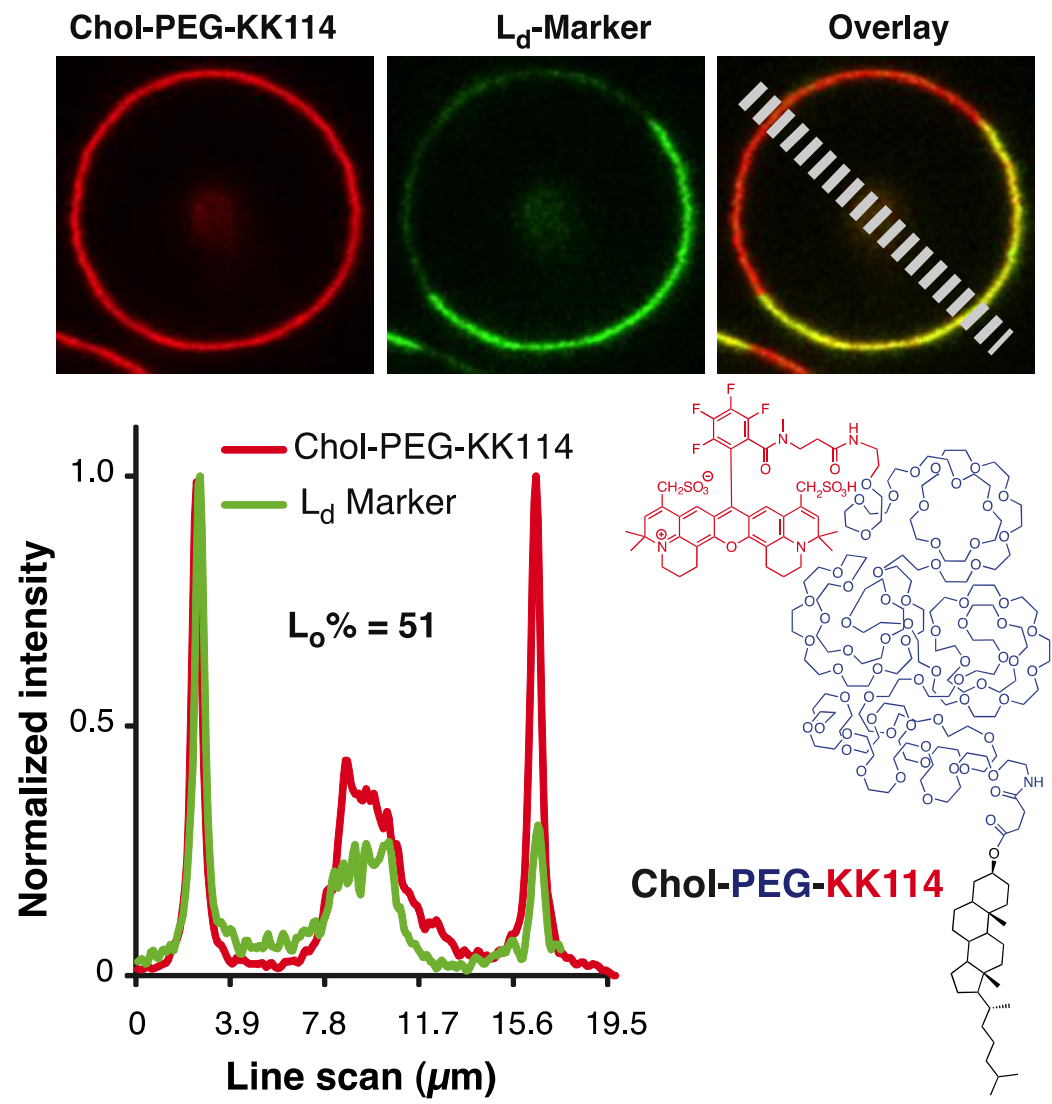

Figure 2-2 Lipid phase partitioning of Chol-PEG-KK114 in GUVs.

A GUV exhibiting $L_{o} / L_{d}$ phase coexistence was doped with the $L_{d}$ marker DiO (green). From the profile the partition coefficient of Chol-PEG-KK114 (structure shown) was determined to be $51 \% \mathrm{~L}_{\mathrm{o}}$. The lower right inset shows the structure of the cholesterol analogue.

\subsubsection{Incorporation of Fluorescent Lipid Analogues into Membrane Sheets}

In order to check the integrity of the membrane sheets after the click reaction, the membranes were labeled with $1 \mathrm{\mu g} / \mathrm{ml}$ octadecyl rhodamine B (R18, Molecular Probes) for 10 minutes; then washed 3 times with PBS (10 minutes each), and embedded in Mowiöl. R18 imaging was performed in confocal microscopy. Alternatively, more specific lipids were used. A stock solution of $50 \mu \mathrm{M}$ of Chol-PEG-KK114 was prepared in pure water containing $1 \%$ ethanol. A $100 \mu \mathrm{M}$ stock solution of PE-Atto647N (N-(Atto647N)-1,2-dipalmitoyl-sn-glycero-3-phosphoethanolamine with headgroup labeling) was prepared in $100 \mu \mathrm{M}$ fat-free BSA water solution (Eggeling et al., 2009). For sphingomyelin (SM) two different fluorescent analogues of sphingosylphosphocholine were used. SM-Atto647N with acyl chain replacement, was purchased from ATTO-TEC and a stock of 10 $\mu \mathrm{M}$ was prepared in fat-free BSA water solution (Eggeling et al., 2009). SM-PEG-KK114 was prepared as described above for Chol-PEG-KK114. The lipid stocks (except R18) were provided by Vladimir Belov and Alf Honigmann. 
For imaging specific lipids, membranes were incubated (after click reactions) with 1:100-1:200 dilutions from the stocks above in PBS for 30 minutes at RT, followed by washing with PBS and embedding in Mowiöl. Samples were imaged directly after preparation.

\subsubsection{Transfer of Fluorescent Probes to Plasma Membranes of Living Cells}

A stock solution of $50 \mu \mathrm{M}$ of Chol-PEG-KK114 was prepared in pure water containing $1 \%$ ethanol. COS-7 cells were then incubated with $200 \mu$ of a 1:200 dilution of the stock in cell medium, for 3 minutes at RT. After incubation cells were washed with medium. WGA was diluted to $100 \mathrm{nM}$ in PBS; cells were incubated with $100 \mu \mathrm{l}$ of this solution for 3 minutes, and then washed with medium.

\subsubsection{Fluorescent Labeling of WGA}

For STED and STED-FCS imaging in living cells, WGA was labeled with the far-red fluorescent dye Star635 (Abberior). $1 \mathrm{ml}$ from $2 \mathrm{mg} / \mathrm{ml}$ solution (pH 8.4) of WGA was incubated with $100 \mathrm{nmol}$ Star635-NHS for $2 \mathrm{~h}$ at RT. Unreacted dye was removed by size exclusion chromatography using a Nap25 column (GE Healthcare). This protocol was kindly applied by Vladimir Belov (MPI-BPC).

\subsection{Endocytic Uptake Assays}

PC12 cells were grown for 3 days under normal conditions or in AHA-incubation medium with AHA. Cells were incubated with $100 \mu \mathrm{g} / \mathrm{ml}$ Dextran-Alexa488 (Molecular Probes) or $50 \mu \mathrm{g} / \mathrm{ml}$ transferrin-Alexa488 (Molecular Probes) for 5 minutes to label early endosomes (EE) at $37^{\circ} \mathrm{C}$ in Ringer buffer. Alternatively, incubations were done with $10 \mu \mathrm{g} / \mathrm{ml}$ choleratoxin B-Alexa594 (Sigma) for 20 minutes or with $100 \mathrm{nM}$ Lysotracker-DND99 (Molecular Probes) for 30 minutes in respective cell medium to label the late endocytic compartments. The cells were washed and either directly fixed (with 4\% PFA for 20 minutes on ice and 25 minutes at RT) or in the case of transferrin let to recycle for 60 minutes and then fixed. Imaging was done with a 40X air objective (NA $=0.75$, Olympus) using an Olympus IX 71 microscope with a computer-operated CCD camera and a $100 \mathrm{~W}$ mercury lamp. Total fluorescence in the cells was analyzed by manual selection of the cell borders and compared for normal and AHA conditions, using a custom-written Matlab routine. 


\subsection{Neuronal Differentiation Assay}

Normal or AHA-treated cells were also given $100 \mathrm{ng} / \mu \mathrm{l}$ human neuronal growth factor (NGF- $\beta$, Sigma) for 3 days. After fixation, R18 was applied to see the morphology of cells and images of long axon-like protrusions was taken. The protrusion length was analyzed as indicative of the neuronal differentiation.

\subsection{Click Labeling of Cell Lysates}

PC12 cells, grown in culture dishes with AHA for 3 days, were scraped off the dish with ice-cold PBS containing protease inhibitors ( $0.2 \mathrm{mM}$ PMSF, $1 \mu \mathrm{g} / \mathrm{ml}$ pepstatin A, leupeptin and aprotinin). Collected cells were centrifuged at $2000 \mathrm{~g}$ for 5 minutes at $4^{\circ} \mathrm{C}$ and the supernatant liquid was discarded. The pellet was lysed in PBS with protease inhibitors, $0.5 \%$ sodium doedecyl sulfate (SDS) and 50 units/ $\mu$ I DNAse I (AppliChem) through vortexing and boiled at $96{ }^{\circ} \mathrm{C}$ for 10 minutes. SDS concentration was brought down to $0.1 \%$ by addition of PBS with a final volume of $0.2 \%$ Triton $\mathrm{X}-100$. The lysate was centrifuged again at $2000 \mathrm{~g}$ for 5 minutes at $4^{\circ} \mathrm{C}$ and the supernatant liquid was collected. Equal amounts were divided into tubes and click reaction was performed with or without Atto647N-alkyne. Then the samples were mixed with sample buffer loaded to a $10 \%$ polyacrylamide (below). For in-gel labeling of all proteins, Coomassie Fluor Orange protein gel stain (Molecular Probes) was applied to the gel.

\subsection{SDS-Polyacrylamide Gel Electrophoresis (PAGE)}

Proteins were separated elecrophoretically using a 10\% denaturing Tris/Tricin SDS-PAGE system as described before (Schägger and Jagow, 1987).

Resolving gel: $10 \%$ bis-acrylamide (Roth), $1 \mathrm{M}$ Tris (pH 8.45), $0.1 \%$ SDS and $10 \%$ glycerol.

Stacking gel: $4 \%$ bis-acrylamide, 1 M Tris (pH 8.45), 0.1\% SDS.

TEMED ( $N, N, N^{\prime}, N^{\prime}-$-Tetramethylethylenediamine) and ammonium persulfate were added to each for polymerization. Electrophoresis was done in a discontinuous buffer system, with anode buffer of $0.2 \mathrm{M}$ Tris- $\mathrm{HCl}, \mathrm{pH} 8.9$ and cathode buffer of $0.1 \mathrm{M}$ Tris, $0.1 \mathrm{M}$ Tricin, $0.1 \% \mathrm{SDS}, \mathrm{pH} 8.25$, at $70 \mathrm{~V}$ for 15 minutes followed by $60-90$ minutes at $120 \mathrm{~V}$.

Coomassie orange staining was done according to manufacturer's protocol (Molecular Probes) and gels were documented using an FLA9000 fluorescence scanner (Fujifilm). 


\subsection{Organelles}

PC12 post-nuclear supernatants (PNS) were produced as described previously (Barysch et al., 2010). Briefly, PC12 cells were grown to $80 \%$ confluency in $14.5-\mathrm{cm}$ dishes in AHA incubation medium for 3 days. Cells were harvested by trypsin/EDTA treatment and collected after centrifugation at $4^{\circ} \mathrm{C}$. The cell pellet was washed several times. It finally resuspended in homogenization buffer with protease inhibitors $(0.2 \mathrm{mM} \mathrm{PMSF}$ and $1 \mu \mathrm{g} / \mathrm{ml}$ pepstatin A, leupeptin and aprotinin) and homogenized using a stainless steel ball homogenizer (Balch and Rothman, 1985) applying 10-20 passages. The resulting homogenate was centrifuged at $4^{\circ} \mathrm{C}$ in a table-top centrifuge (Eppendorf 5417R) at $1200 \mathrm{~g}$ for $15 \mathrm{~min}$. The supernatant (PNS) was collected, divided into single-use aliquots, snap-frozen and stored at $-80^{\circ} \mathrm{C}$ until use. The protein concentration was determined by the Bradford assay.

For studying endosomes, EE fractions were enriched from freshly obtained PNS by discontinuous sucrose gradient ultracentrifugation (in a Sorvall Discovery $90 \mathrm{SE}$ centrifuge) at $4^{\circ} \mathrm{C}$ for 90 minutes at 35,000 rpm using a TH641 rotor (Sorvall). A band (at the $25 \%-35 \%$ sucrose interface) highly enriched in EE was collected as described before (Bethani et al., 2009). EE fractions were divided into single-use aliquots, snap-frozen and stored at $-80^{\circ} \mathrm{C}$ until use. The protein concentration was determined by the Bradford assay.

For the experiment, organelles were diluted in cold PBS and centrifuged onto coverslips (Barysch et al., 2010) for 45 minutes in an Eppendorf $5810 \mathrm{R}$ centrifuge at $4^{\circ} \mathrm{C}$ and $2683 \mathrm{~g}$. EE fractions were incubated in vitro with $45 \mathrm{mM} \mathrm{MBCD}$, for 30 minutes at $37^{\circ} \mathrm{C}$. For studying mitochondria, the PNS fractions were incubated in vitro with $5 \mathrm{mM}$ cholesterol-MBCD complex (Christian et al., 1997) for 30 minutes at $37^{\circ} \mathrm{C}$, to load cholesterol into their membranes. Afterwards, the samples were washed and fixed, and AHA moieties were coupled to fluorescent dyes by click reactions. To identify mitochondria in the PNS specifically, samples were immunostained for the cytosolic mitochondrial epitope TOMM20 (using mouse anti-TOMM20, Sigma, WH0009804M1).

\subsection{Confocal and STED Imaging}

Fluorescence images were acquired with a Leica TCS SP5 STED confocal microscope (Leica) equipped with a $100 \times 1.4$ N.A. HCX PL APO CS oil objective (Leica) using the Leica Application Suite Advanced Fluorescence software. In STED mode, the excitation of Atto647N was performed using a pulsed diode laser (Leica Microsystems) at $635 \mathrm{~nm}$, and depletion was achieved by means 
of a Spectra-Physics Mai Tai tunable laser (pulsed at $80 \mathrm{MHz}$; Titanium Sapphire, Newport Spectra-Physics) at $750 \mathrm{~nm}$, with an output power of $1.6 \mathrm{~W}$. For 2-color STED, Chromeo494 was excited with a pulsed diode laser at $535 \mathrm{~nm}$ and the same depletion laser was used for STED effect. For multichannel confocal images the following excitation lines were used: an Argon laser at a wavelength of $488 \mathrm{~nm}$ for GFP or Cy2, and Helium-Neon lasers at $594 \mathrm{~nm}$ for Cy3 and $633 \mathrm{~nm}$ for Atto647 or STAR635. Appropriate emission intervals were selected using an AOTF filter (Leica) and line by line sequential scanning at $1000 \mathrm{~Hz}$ was applied with 32 times line averaging for confocal and 96 times line averaging for STED. Signal detection was performed using photomultiplier tubes in confocal mode and an avalanche photodiode in STED mode. Laser intensities were adjusted to obtain the best signal-to-noise ratios for each experiment; the images are displayed with individual scaling, except for cases to be directly compared (such as in Figure 3-4C and Figure 3-10).

The resolution was $\sim 40-50 \mathrm{~nm}$ when measuring Atto647N (displayed in red in overlay images, except neuronal stainings). For 2-color STED, measurements with Chromeo494 (displayed in green in overlay images) were performed at a resolution of $\sim 80 \mathrm{~nm}$.

\subsection{STED-FCS Measurements and Data Analysis}

STED-FCS measurements and analyses were performed in collaboration with by Alf Honigmann. The STED-FCS setup and the data analyses method used for the experiments have been previously described (Eggeling et al., 2009; Honigmann et al., 2013a). Briefly, the measurements were made at random locations on the upper cell membrane of cultured cells for 10 seconds. The STED power applied in the back aperture of the objective was $50 \mathrm{~mW}(780 \mathrm{~nm})$, resulting in an observation spot with a diameter of $80 \mathrm{~nm}$ (FWHM). The excitation power was $8 \mu \mathrm{W}$ for the Chol-PEG-KK114 measurements and $2 \mu \mathrm{W}$ for the WGA-Star635 measurements. For the 2-color experiments with WGA-Alexa488 and Chol-PEG-KK114 the excitation power for the $488 \mathrm{~nm}$ laser was $0.1 \mu \mathrm{W}$, to reduce bleaching during the 10 -second measurements.

To find out if diffusion of the WGA-Star635 and Chol-PEG-KK114 was faster or slower at sites where WGA was enriched (protein clouds) two different types of analyses were performed. From every FCS measurement the lateral diffusion coefficient of the probe (WGA-Star635 or Chol-PEGKK114) and the average intensity of WGA (WGA-635 or WGA-Alexa488) were obtained. First, Pearson's correlation coefficient (PCC) was calculated from the distribution of the WGA intensities and that of the respective lateral diffusion coefficients. The values of the PCC can range from 1 to 
-1 meaning a strong positive correlation (diffusion is faster in protein clouds) or a strong negative correlation (diffusion is slower inside the protein clouds) respectively. When PCC $=0$ there is no correlation between WGA intensity and diffusion coefficient. For example, $P C C=-0.5 \pm 0.07$ was reached for WGA, which indicates that diffusion inside the clusters tends to be slower. Second, the diffusion coefficients were classified according to the intensity of WGA. The WGA brightness was normalized (0-1) and the diffusion coefficients of the spots with the $40 \%$ highest WGA intensities (seen as "diffusion in protein clusters/clouds") and $40 \%$ lowest intensity ("diffusion out of protein clusters/clouds") were separated (Figure 2-3). From these two classes the average diffusion coefficient and standard deviation were calculated to estimate if the diffusion was significantly different. This analysis showed that diffusion of glycosylated proteins and lipids inside the clusters was slower than outside. Note that FCS is sensitive only to the mobile fraction of molecules. Therefore this analysis is a lower estimate and it omits all molecules that are very slow $\left(D_{\text {lat }}<0.01\right)$ or non-moving.

The same type of analysis was performed with the fluorescent cholesterol analogue Chol-PEGKK114. For this the protein clouds were stained with green fluorescent WGA (WGA-Alexa488) and the diffusion of the red fluorescent cholesterol was measured simultaneously. While the distribution of Chol-PEG-KK114 itself in the plasma membrane was rather homogenous, the STEDFCS analysis revealed again a negative correlation between the diffusion coefficient of the cholesterol analogue and the distribution of WGA (PCC $=-0.4 \pm 0.07$ ). Accordingly, the diffusion of Chol-PEG-KK114 was significantly slower inside of the protein clouds (1.3-fold). As expected the mean diffusion coefficient of the cholesterol analogue was 10 times faster than the mobility of WGA-bound proteins and lipids.
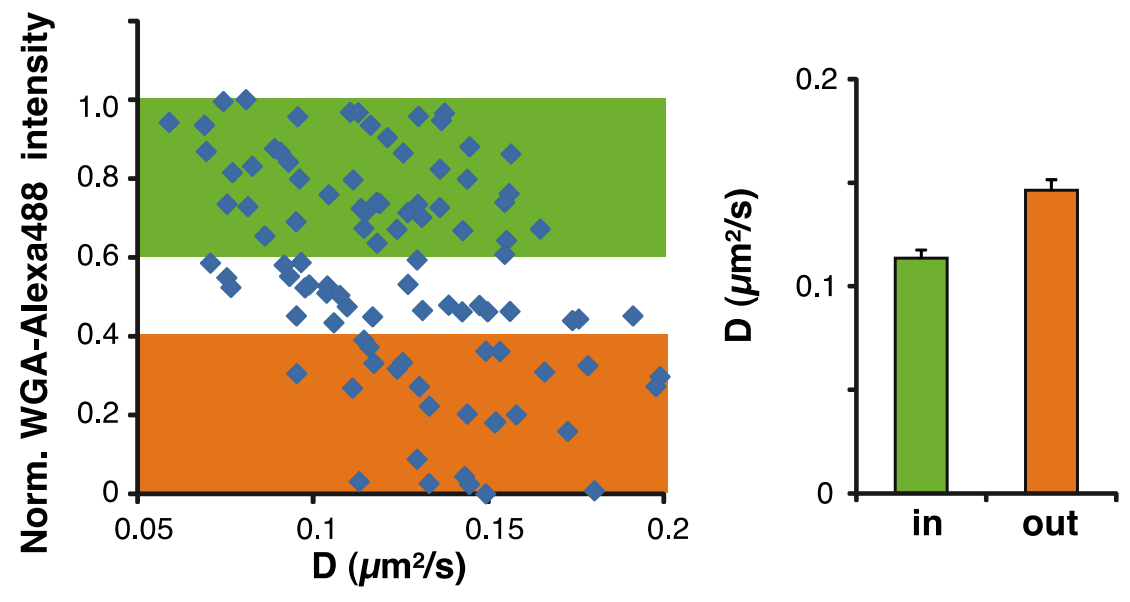

Figure 2-3 In and out of WGA cluster classification.

In cluster was defined as all diffusion coefficients that were measured at positions with a WGA intensity of $>60 \%$ (the $40 \%$ highest intensity fraction), out of cluster was defined accordingly as the $40 \%$ lowest intensity fraction. The analyses were performed by Alf Honigmann. 


\subsection{Sample Preparation and Imaging for SIMS and COIN}

\subsubsection{Preparation of Hippocampal Neurons for Imaging of Organellar Turnover}

Primary cultures of hippocampal neurons were prepared (by Christina Schäfer) from newborn rats, plated onto astrocytic monolayers as described before (Willig et al., 2006) and used after 10 days in vitro. The cultures were incubated in Neurobasal-A medium (GIBCO) with 2\% B27 Supplement (GIBCO), 1\% GlutaMAX Supplement (GIBCO) and 60 units/ml Penicillin-Streptomycin (Lonza) in the presence of $2.2 \mathrm{mM}{ }^{15} \mathrm{~N}$-leucine for 3 days. The cells were fixed for 45 minutes at RT with $4 \%$ PFA, and quenched for 25 minutes with $100 \mathrm{mM} \mathrm{NH}_{4} \mathrm{Cl}$, then permeabilized with $0.1 \%$ Triton- $\mathrm{X} 100$ in PBS containing 1.5\% BSA.

The cells were incubated for $1 \mathrm{~h}$ with the given dilutions of the following primary antibodies in $0.1 \%$ Triton-X100 in PBS containing 1.5\% BSA.

\section{For axonal staining:}

- mouse monoclonal anti-TOMM20 (1:100, Sigma, WH0009804M1),

- guinea pig synaptophysin 1 (1:500, Synaptic Systems, 101004) and

- rabbit polyclonal anti-bassoon (1:400, Synaptic Systems, 141002)

\section{For neuronal cell body staining:}

- mouse monoclonal anti-TGN38 (1:100, BD Biosciences, 610898),

- guinea pig synaptophysin 1 (1:500, Synaptic Systems, 101004) and

- rabbit polyclonal anti-calnexin (1:100, Abcam, 22595).

After several washes, primary antibodies were detected by incubating the cells for $1 \mathrm{~h}$ with 1:100 dilutions of the following secondary antibodies in $0.1 \%$ Triton-X100 in PBS containing 1.5\% BSA:

- goat anti-mouse Cy2 (1:100, Dianova, 115-225-146),

- donkey anti-guinea pig Cy3 (1:100, Dianova, 706-165-148) and

- Atto647N-labeled goat anti-rabbit (1:100, Rockland, 611-156-122S).

Then cells were washed with PBS and a post-fixation step was performed with $4 \%$ PFA and $0.2 \%$ glutaraldehyde in PBS for 30 minutes at RT, followed by quenching with $100 \mathrm{mM} \mathrm{NH}_{4} \mathrm{Cl}$ in PBS for 15 minutes. 


\subsubsection{Preparation of COS-7 cells for imaging of membrane patterning}

The membranes of COS-7 cells were labeled with a fixable membrane dye by 5-minute incubation on ice in Ringer buffer. The cells were washed with ice-cold Ringer buffer and were fixed with $4 \%$ PFA and $0.2 \%$ glutaraldehyde in PBS for 15 minutes on ice and 30 minutes at RT, followed by quenching with $100 \mathrm{mM} \mathrm{NH}_{4} \mathrm{Cl}$ in PBS for 25 minutes and LR White embedding.

\subsubsection{LR White Embedding}

Both neuronal and COS-7 samples were embedded in LR White resin (London Resin Company). First, partial serial dehydration was performed with ethanol: 30\% ethanol for 5 minutes, 3 times $50 \%$ ethanol for 5 minutes each, and incubation with 1:1 mixture of $50 \%$ ethanol and LR White (London Resin Company Ltd) for 30 minutes. This was followed by the incubation of coverslips with LR White in ethanol-free conditions for $1 \mathrm{~h}$. The cells were then embedded at $4^{\circ} \mathrm{C}$ with LR White and accelerator mixture (London Resin Company), and incubated for 15 minutes. Afterwards they were incubated for 90 minutes at $60^{\circ} \mathrm{C}$, and cooled down to RT. For neuronal cultures, 200-nm sections were cut from the resulting blocks using an EM UC6 ultramicrotome from Leica Microsystems. For membrane imaging, 100-nm sections were cut. The sections were deposited on silicon wafers as described in (Zhang et al., 2012).

\subsubsection{COIN imaging of Neurons}

First, fluorescence images were acquired with a Leica TCS SP5 STED confocal microscope as described above. Cy2, Cy3 and Atto647N channels were acquired using the Argon laser at a wavelength of $488 \mathrm{~nm}$ and the Helium-Neon lasers at $594 \mathrm{~nm}$ and $633 \mathrm{~nm}$, respectively, in confocal mode. This was followed by STED imaging of Atto647N with the a pulsed diode laser at $635 \mathrm{~nm}$; depletion was achieved by means of a Spectra-Physics Mai Tai tunable laser at $750 \mathrm{~nm}$, with an output power of $1.6 \mathrm{~W}$. Images of $2048 \times 2048$ pixels were taken at $3 \times$ zoom at a final pixel size of $25.2 \mathrm{~nm}$ with $1000 \mathrm{~Hz}$ scanning. After imaging, the area around the imaged cell was marked by burning the resin material with the Mai Tai laser described above.

Then same areas were identified in brightfield on a NanoSIMS $50 \mathrm{~L}$ setup (Cameca). For SIMS imaging, a ${ }^{133} \mathrm{Cs}^{+}$primary ion beam at $16 \mathrm{keV}$ energy was used to sputter and partly ionize atoms belonging to the sample. These ions were then mass separated and detected by using a double focalization mass spectrometer equipped with 7 miniature electron multipliers (Hamamatsu). The 
mass resolving power was adjusted to suppress interferences at all masses (allowing the optimal separation of ${ }^{12} \mathrm{C}^{15} \mathrm{~N}^{-}$from interfering ions like ${ }^{13} \mathrm{C}^{14} \mathrm{~N}^{-}$). Primary ion beam current was 0.5 to $1 \mathrm{pA}$, scanning parameters were $512 \times 512$ pixels $(12.88 \times 12.88 \mu \mathrm{m})$ with a dwell time of $500 \mu$ s per pixel. SIMS imaging was performed in collaboration with Angela Vogts (Leibniz-Institute for Baltic Sea Research).

\subsubsection{COIN imaging of Cell Surfaces}

Cell surface areas in the sections were identified by confocal imaging of the membrane dye using a $633 \mathrm{~nm}$ laser for excitation. The imaged areas then were marked as for neurons and identified in the SIMS setup. Only the regions fluorescently identified to be membranes were imaged by SIMS. Images of $512 \times 512$ pixels were scanned (12.88x12.88 $\mu \mathrm{m}$ in size). The dwell time was $250 \mu$ ser pixel. 12-40 images were obtained for each area and were aligned and summed afterwards to generate the resulting images.

\subsection{Image Analysis}

All analyses were performed with the help of Matlab software (Mathworks), using self-written routines (mostly developed by Silvio Rizzoli).

Cloud size quantification (Figure 3-3D): Protein clouds were identified as the areas whose intensity was above the background intensity (selected on empty coverslip areas). For accurate identification, images were first filtered using median and averaging filtering. An automatic threshold (empirically devised) was applied to determine the areas with intensities above background and to generate masks for each individual cloud. Size identification was performed automatically by fitting Lorentzian curves to the mask areas and determining the full-width-athalf-maximum (FWHM). For non-circular structures, the largest diameter was taken as the size value. The cloud area quantification for Figure 3-12 was performed similarly, although numbers are expressed in terms of areas, rather than diameters.

Line scans for 2-color STED images: Line scans (1.23 $\mu \mathrm{m}$ in length, 3 pixels in width) were performed manually on the protein clouds and were subsequently averaged. The line scans were centered on the midpoints of protein clouds. All curves were normalized to the baseline signal, obtained from the average line scans (the region flanking the peaks). Typically 30-50 line scans 
were obtained from one membrane sheet. For analysis all line scans from several membrane sheets were pooled together.

Quantification of membrane area occupied by clouds (Figure 3-11): For a simple indication of protein clustering on the plasma membrane, the space occupied by the protein clouds was calculated and expressed as percentage of the total membrane area. This measurement, termed "area occupied by clouds" in the figures, is insensitive to changes in the structure of protein clouds, but reports accurately any changes in the relative proportions of clouds and protein-poor areas. The values were obtained by selecting rectangular regions of interest and measuring the fraction of the pixels whose fluorescence intensity was clearly above the background staining intensity (within more than two standard deviations from the mean background intensity; the background was selected on empty coverslip areas adjacent to the membrane sheets). All values are presented in the figures as percentage of the relevant controls (which were incubated with buffer alone under the same conditions). To find protein-poor area (in Figure 3-14), the same analysis was performed and the area of the membrane sheets that was within the range of background staining was calculated.

Intensity in the area between clouds (Figure 3-10): The protein clouds and the areas outside of protein clouds were determined as described above for cloud size quantification. Protein intensity in each category was then automatically detected for all regions of interest within one image, and region values were averaged to obtain representative data.

Protein clustering in organelles (Figure 3-15): Line scans were drawn manually along the membranes of the organelles, and the coefficient of variation of the intensity along the line scan (standard deviation divided by the mean) was calculated. This value is a direct indication of protein clustering, since it reports the magnitude of the differences between the peak signals (clusters) and the baseline. For display, fluorescence images were deconvolved with Huygens Essential software (Scientific Volume Imaging), using the inbuilt routines (applying classical maximum likelihood estimation algorithm) designed specifically for STED images.

Cloud centerness (Figure 3-24): Cloud center preference was determined as follows, from the average line scans drawn over immunostained protein clouds. The ratio between the intensity of the immunostained protein and the intensity of the protein cloud was determined at two points: 1) the cloud center (Protein intensity center/Cloud intensity $_{\text {center }}$ ), and 2) the half-width point of the 
cloud, representing the edge (Protein intensity edge/Cloud intensity $_{\text {edge }}$ ). Cloud center preference was then obtained by dividing the first ratio (from the center) by the second one (from the edge).

Circular model clouds (Figure 4-1): The line scans for general and specific protein stainings were used to generate circular, idealized protein domains, as follows. Matrices were generated for each protein of interest, composed of 61 line scans that were randomly selected from all the line scans obtained for that particular protein. 360 such matrices, each containing a different set of line scans, were generated. Each matrix was rotated by an angle between $1^{\circ}$ and $360^{\circ}$. The sum of all rotated matrices forms the image of a circular protein distribution.

For COIN imaging of neurons: the fluorescence images were rotated and aligned to the SIMS images. A background subtraction was performed for the ${ }^{15} \mathrm{~N} /{ }^{14} \mathrm{~N}$ ratio images, for display purposes. All line scans (horizontal) and spot size measurements were performed using customwritten routines in MATLAB. Several lines were averaged to increase the signal-to-noise ratio (3 lines for Figure 3-25; 5 lines for Figure 3-27 and Figure 3-28). Fluorescence images were deconvolved with the aid of Huygens Essential software (applying classical maximum likelihood estimation algorithm) using the inbuilt routines designed specifically for confocal and STED images.

\subsection{Statistics}

All data are presented as mean \pm SEM, unless otherwise noted. For statistical testing student's $\mathrm{t}$ test (unpaired) was applied using SigmaPlot (Mathworks) and p-values were obtained. Significance is denoted as non-significant (ns), when $\mathrm{P}>0.05$; as *, when $\mathrm{P}<0.05$; as **, when $\mathrm{P}<$ 0.01 and as $* * *$, when $\mathrm{P}<0.001$. 


\section{RESULTS}

\subsection{Metabolic Labeling of Cells}

To visualize the general organization of membrane proteins one needs to label a representative fraction of all proteins indiscriminately and to detect them with sufficient sensitivity and resolution. I relied on the metabolic incorporation of a modified amino acid, L-Azidohomoalanine (AHA), which replaces endogenous methionine in all newly produced proteins (Dieterich et al., 2007). Rat neuroendocrine cells (PC12) were grown with AHA for 72 hours, which is long enough for more than 3 complete cell-cycles (Yen et al., 2008), so that a substantial population of proteins can incorporate it. The incubation was done in medium lacking natural methionine and with serum depleted of amino acids through dialysis. The profile of AHA incorporated proteins in cell lysates in comparison to labeling of proteins by coomassie orange is displayed in Figure 3-1. It is expected that the representation of the two populations will be slightly different as coomassie orange detects the SDS bound to the proteins, whereas AHA is incorporated only at the methionine sites, however, protein bands of various sizes can be seen with AHA labeling.

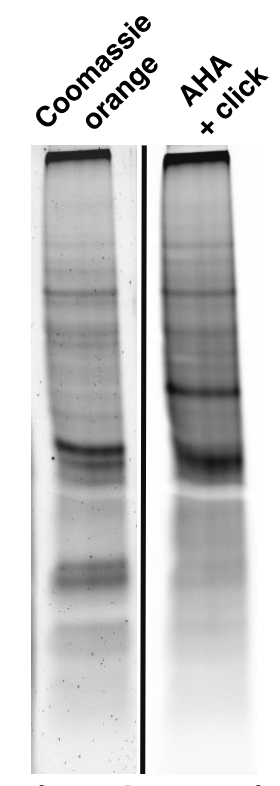

Figure 3-1 Protein labeling profile of cell lysates.

PC12 cells, grown with AHA for 3 days, were lysed and their proteins were extracted. Click reaction was performed in the tube with Atto647N-alkyne. Then the samples were loaded to a $10 \%$ SDS-PAGE gel. For ingel labeling of all proteins, coomassie orange was applied to the gel.

This rather long incubation with AHA and dialyzed serum causes a slight decrease in the growth rate. To check the possible negative effects on cellular metabolism, I have checked the trafficking parameters by performing uptake assays. Varying the incubation time and the molecule for 
uptake, trafficking to early and late endocytic compartments was checked. The labeling of AHAinucbated cells was compared to control cells after incubations with fluorescent transferrin, cholera toxin B, dextran, and Lysotracker. In each case the distribution and labeling levels were similar qualitatively and quantitatively (Figure 3-2A).

In a second assay the differentiation ability of the AHA-incorporated cells was assessed. In this case, neuronal differentiation of PC12 cells was induced by neuronal growth factor (NGF), which stimulates elongation of axon-like protrusions. The lengths of such protrusions were compared to the cells grown under normal conditions with NGF and similar values were obtained (Figure 3-2B).

Additionally, metabolically labeled cells were able to express and correctly target both endogeneous and exogeneous proteins from plasmids (results for immunostainings for more than 20 different proteins, including soluble and membrane proteins, can be seen in the following sections). These results show that metabolically labeled cells maintained normal parameters with regard to organelle and protein distribution, membrane trafficking, and retained their ability to differentiate to a neuronal-like phenotype upon induction with NGF.

A

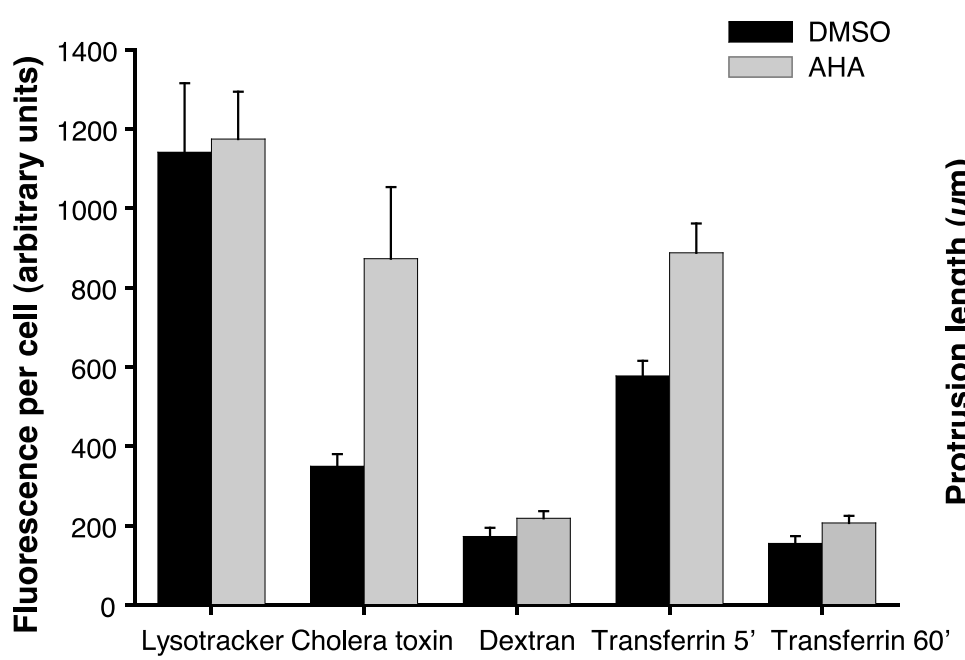

B

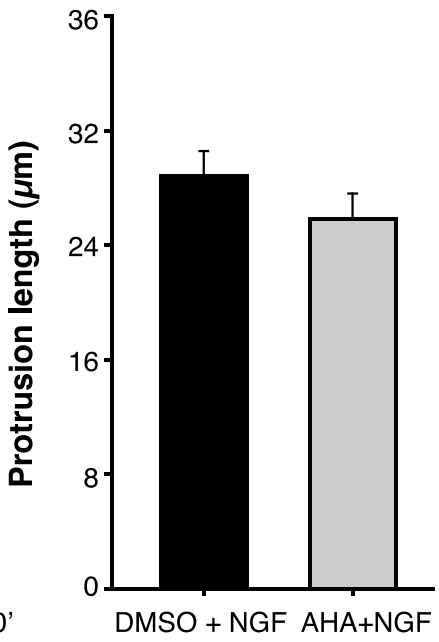

Figure 3-2 Assaying the overall fitness of metabolically labeled PC12 cells.

A. PC12 cells were grown for 3 days under normal conditions or with AHA. Uptake assays were performed by incubating the cells with $100 \mathrm{\mu g} / \mathrm{ml}$ Dextran-Alexa488 for 5 minutes, $100 \mathrm{nM}$ Lysotracker-DN99 for 30 minutes, $10 \mu \mathrm{g} / \mathrm{ml}$ cholera-toxin B-Alexa594 for 20 minutes and $50 \mu \mathrm{g} / \mathrm{ml}$ transferrin-Alexa488 for 5 minutes at $37^{\circ} \mathrm{C}$. Then the cells were washed and either directly fixed (with $4 \%$ PFA for 20 minutes on ice and 25 minutes at RT) or in the case of transferrin let to recycle for 60 minutes. B. Normal or AHA-treated cells were incubated with $100 \mathrm{ng} / \mu \mathrm{l} \mathrm{NGF}$ for 3 days (in parallel to AHA incubation). After fixation, a fluorescently-labeled lipid (R18) was applied to see the morphology of cells and images of long axon-like protrusions was taken. The protrusion length was analyzed as indicative of the neuronal differentiation. 


\subsection{The Protein Clouds}

To be able to investigate the distribution of the proteins associated with the plasma membrane in isolation, without interference from the rest of the cell, I have used a sonication procedure that isolates the substrate-attached membranes, resulting in plasma membrane sheets as detailed in Section 1.4.1 (Avery et al., 2000; Lang et al., 2001). These membranes were fixed and the AHA moieties were coupled to fluorescent molecules by click-chemistry (Dieterich et al., 2007; 2010). This procedure, depicted in Figure 3-3A, ensures that the large-scale fluorescent modification of proteins does not interfere with their normal behavior and function, because it is applied only after membrane fixation.

To reach the optical resolution necessary for the investigation of the nanoscale organization of the membrane we relied on super-resolution stimulated emission depletion (STED) microscopy (Hell and Wichmann, 1994; Willig et al., 2006), which reduces the focal area to $40-50 \mathrm{~nm}$ in the $X-Y$ plane. STED imaging revealed that the protein distribution was not homogeneous; protein clusters aggregated in high-abundance domains that were loosely interconnected (Figure 3-3B). These domains occupied about $50-60 \%$ of the membrane surface, and contained the large majority of membrane proteins ( $81 \pm 13 \%$, mean \pm SEM, 4 independent experiments; compare with the conventional microscopy view in Figure 3-3C). I chose the term "protein clouds" for the high-abundance protein domains. The rest of the membrane was organized as a protein-poor labyrinth that surrounded the clouds (Figure 3-3B). The majority of the protein clouds were $80-$ $200 \mathrm{~nm}$ in diameter (Figure 3-3D). On average, the peak intensity of the clouds was approximately 3-fold higher than that of the neighboring protein-poor areas $(3 \pm 0.25$, mean \pm SEM, 4 independent experiments). 


\section{A}

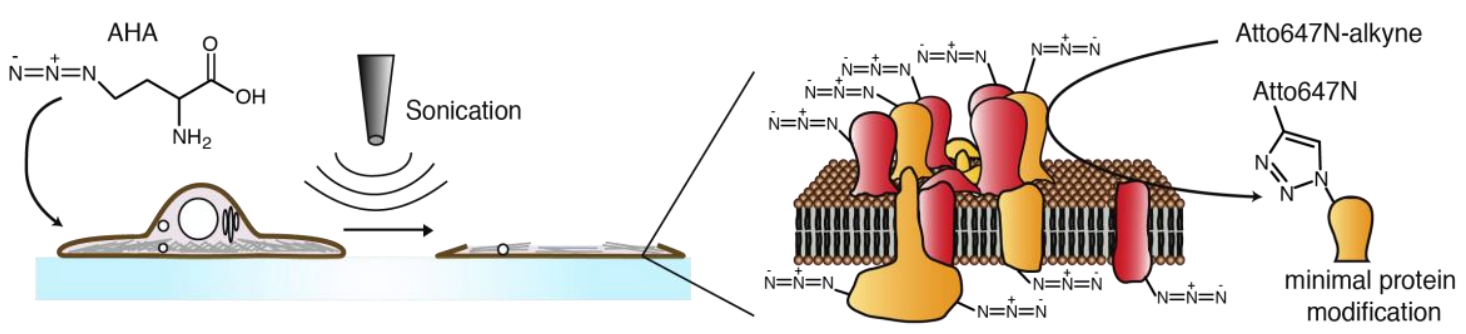

B

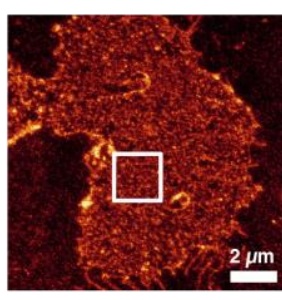

C

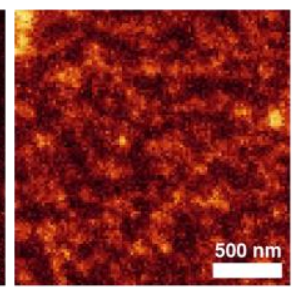

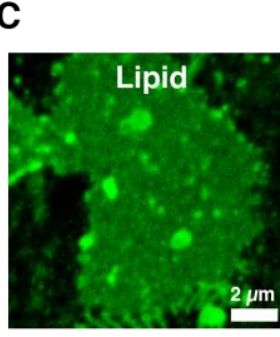

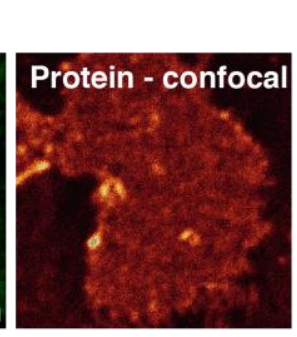

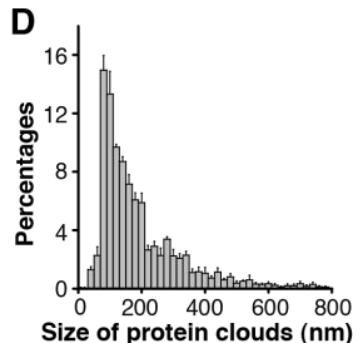

Figure 3-3 Proteins in the membrane are organized into protein clouds.

A. The general principle of the experiments: the amino acid L-azidohomoalanine (AHA) is metabolically incorporated into cells for 72 hours before sonication and plasma membrane sheet generation. The sheets are then fixed and AHA moieties are coupled to alkyne-functionalized variants of fluorescent dyes such as Atto647N or Chromeo494 through click reaction. B. Protein organization in PC12 membrane sheets investigated by STED microscopy. The image on the right shows a high-zoom view of the area marked with the white square. C. The lipid labeling (using R18) and the confocal image of the membrane in panel B. R18 labeling was performed as the last step before embedding, to check for the lipid integrity of the membrane sheets. The very bright spots are very likely the vesicles still attached to the membrane; therefore such areas are avoided while analyzing the images. D. The graph indicates a size histogram of protein clouds (mean \pm SEM, from 5 independent experiments).

To check whether the cloud distribution was induced artifactually by coupling the proteins to the fluorescent dye after PFA fixation, I have repeated the labeling after a harsher fixation step with 4\% PFA and $0.2 \%$ glutaraldehyde, which was shown to give the minimal residual protein movement after fixation (Tanaka et al., 2010). As shown in Figure 3-4A, the distribution was similar. An additional control was to check the effect of sonication. For that the cells were first fixed with 4\% PFA and $0.2 \%$ glutaraldehyde and then sonicated, which should reflect the cellular status before sonication (Figure 3-4B). Alternatively, fixation was avoided completely and the click reaction was done on native sheets (Figure $3-4 C$ ). The reaction was also demonstrated to specifically label proteins by the massive loss of fluorescence upon trypsinization (Figure 3-4C). These controls show that the cloud pattern was not induced by the particular preparation procedure.

An additional concern was that the pattern could have been induced by the attachment of the membrane to the glass surface. We therefore investigated the top membranes of whole cells, by embedding them in a plastic resin and cutting ultrathin sections ( $10 \mathrm{~nm}$ thick). We imaged the upper membrane of cells in such sections, and observed the same pattern: high-abundance 
protein domains separated by narrow protein-poor areas (Figure 3-4D). Finally, similar patterns were observed in membrane sheets from unrelated cells, such as primate fibroblasts, COS-7 (displayed later in Figure 3-16).
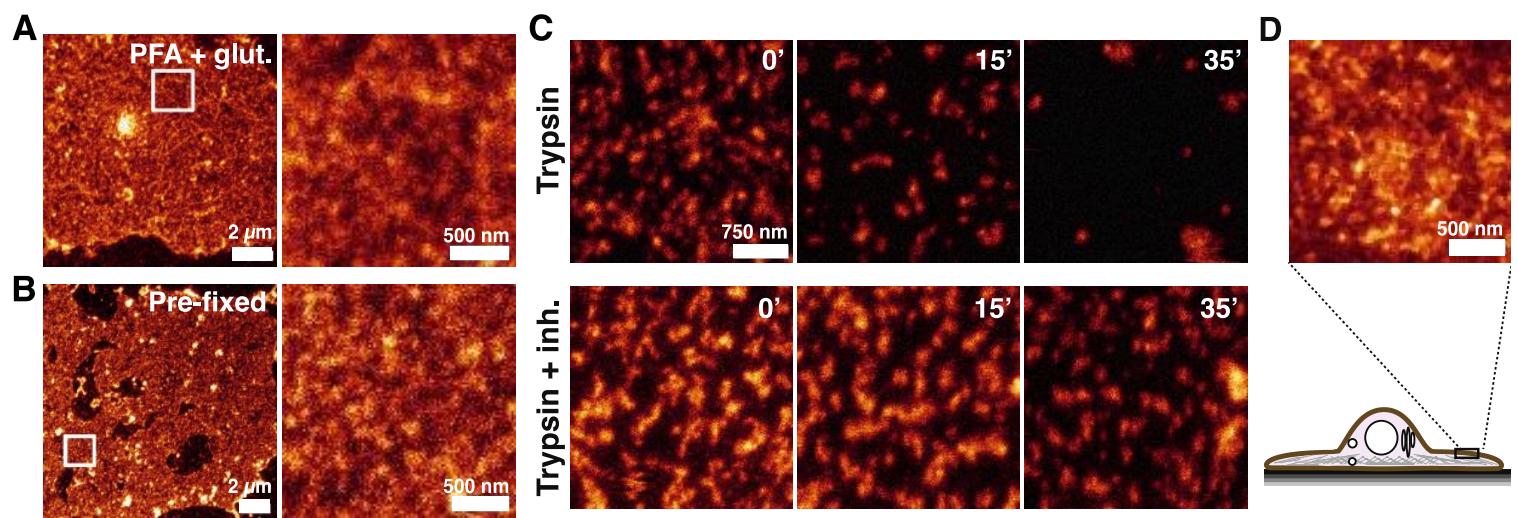

Figure 3-4 Protein clouds under different sample preparation conditions.

A. Representative STED images of a PC12 sheet click-labeled after fixation with $4 \%$ PFA and $0.2 \%$ glutaraldehyde for 45 minutes at RT. The right panel shows a high-zoom view of the area marked with the white square. B. Alternatively, the cells were fixed with $4 \%$ PFA and $0.2 \%$ glutaraldehyde for $45 \mathrm{~min}$ at RT, and quenched with $\mathrm{NH}_{4} \mathrm{Cl}$ for 25 minutes. Only then sonication was applied to obtain sheets. C. STED images of click-labeled, unfixed PC12 membrane sheets treated with $1 \mathrm{mg} / \mathrm{ml}$ trypsin or with $1 \mathrm{mg} / \mathrm{ml}$ trypsin and $2 \mathrm{mg} / \mathrm{ml}$ trypsin inhibitor (shown in upper or lower panels, respectively). Images were taken just before the addition of trypsin or trypsin + inhibitor ( 0 minutes), and at 15 and 35 minutes after the addition. Images of different membranes are shown to avoid photobleaching. Image intensity is scaled identically for all frames. D. Protein organization in membranes of whole cells that were fixed and coupled to Atto647N through the click reaction, before being embedded in a plastic resin and cut into ultrathin sections $(10 \mathrm{~nm})$. The STED image was processed using a median filter, to reduce noise.

In order to make sure that the cloud pattern is not arising from the fluorescent labeling and imaging related artifacts, I wanted to apply a label-free detection technique. For that I have turned to a mass-spectrometry based method for isotopic imaging, secondary isotope mass spectrometry (SIMS). This method has been recently used in important studies for detection of lipids with stable isotopes in cellular membranes (Frisz et al., 2013a; 2013b) and proteins in specialized cells (Zhang et al., 2012) based on morphology. However, since we wanted to focus only on proteins in the membrane, we needed the light microscopy to find the flat membrane areas to be imaged in SIMS. A correlation method for light microscopy and SIMS was not technically established, therefore I have developed a scheme for correlated optical isotopic nanoscopy (COIN). The development and application of COIN are described as a technical note in Section 3.10. Here, only the relevant results, SIMS imaging of proteins in the membranes, are presented.

SIMS identifies isotopes instead of fluorescent molecules and thus allows the label-free characterization of membrane patterning. Since proteins contain the majority of nitrogen atoms in the membrane, I have analyzed the nitrogen signal $\left({ }^{14} \mathrm{~N}\right)$ as an indication of protein patterning. 
The ${ }^{14} \mathrm{~N}$ distribution, measured on the upper membrane of cells after plastic embedding and thin sectioning, was heterogeneous, composed of high-density domains on a lower background (Figure 3-5). This distribution was compatible with the protein clouds determined by STED microscopy, notwithstanding the differences in sensitivity and resolution between the two techniques (STED has a 40-50 $\mathrm{nm}$ lateral resolution and $\sim 500 \mathrm{~nm}$ axial resolution; the lateral resolution of the SIMS images we obtained was $\sim 100 \mathrm{~nm}$, with axial resolution of 7-10 nm).
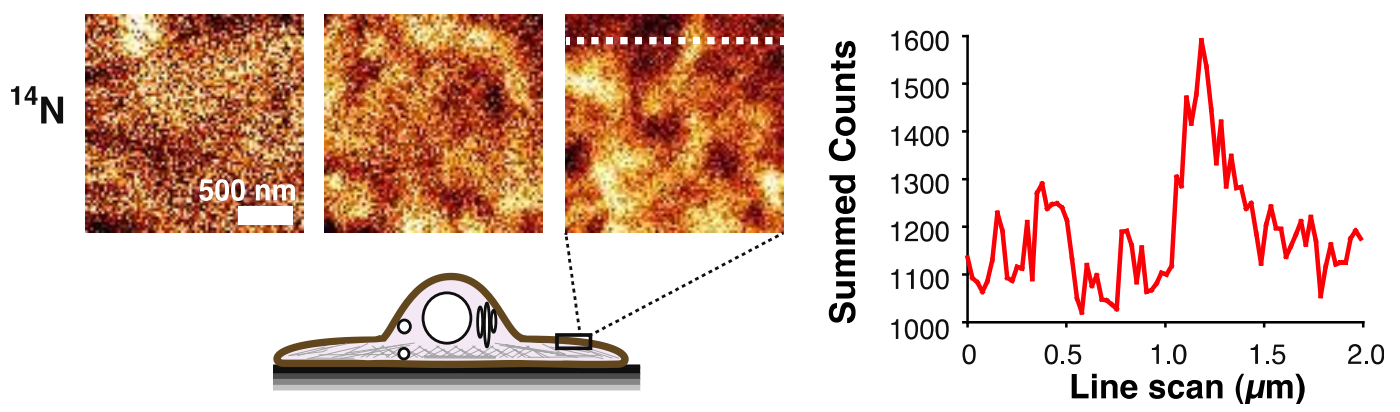

Figure 3-5 Label-free imaging of proteins by SIMS

SIMS imaging was performed on the surface membranes of COS-7 cells, to detect the distribution of ${ }^{14} \mathrm{~N}$ in the membranes. Three example SIMS images are shown. ${ }^{14} \mathrm{~N}$ reports mainly protein distribution in this experiment, since proteins contain most of the membrane nitrogen (also the signal contribution from lipids is partially lowered further by potential extraction of lipids during the dehydration and plastic embedding required for SIMS). Cells were fixed, embedded in LR White, cut into ultrathin sections (100 nm) and adsorbed onto silicon wafers, and imaged with a SIMS instrument. Scale bars are $500 \mathrm{~nm}$. A line scan (5 pixel width) is presented, performed over the white dashed line, to depict the heterogeneity of the signal. SIMS imaging was performed in collaboration with Angela Vogts (Leibniz-Institute for Baltic Sea Research).

The controls presented so far shows that the protein cloud organization can be reproduced under different preparation conditions. For the ease of handling we have used the condition described in Figure 3-3A-B as a general strategy for the future experiments, that is cells were first sonicated, then fixed with 4\% PFA (occasionally with the addition of glutaraldehyde where indicated) and next click reaction was performed. This condition minimizes the leftover cytoplasmic material, provides good antigenicity for antibodies, avoids application of a copper-catalyzed reaction on native sheets and evades long-term incubation of native sheets under artificial conditions.

The last point here is important because if the native sheets are incubated too long without fixation, protein aggregation was observed to occur probably due to loss of some molecules in time, as was also reported previously (Frick et al., 2007). In Figure 3-4B, where click reaction was applied on unfixed sheets (which means an incubation time of $\sim 45$ minutes before the start of trypsinization, it is possible to see larger protein-poor areas in the control conditions, potentially because of this partial aggregation and loss of peripheral proteins over time. Therefore in cases 
where it was necessary to apply a treatment to membrane sheets before fixation (as for Section 3.8), the incubation durations were minimized and always kept below 20 minutes.

\subsection{Labeling Proteins of Different Ages}

Next, I have proceeded to investigate the composition of the protein clouds, using PC12 membrane sheets as a model. The sizes of most of the protein clouds are larger than a typical membrane protein cluster dominated by one single type of protein. This suggests that they might be multi-protein aggregates. I first tested whether proteins of different ages accumulate to the same clouds, by incubating the cells with two different amino acid analogues, AHA and homopropargylglycine (HPG), for different amounts of time. AHA was incorporated into the cells for $72 \mathrm{~h}$, and thus labeled most of the cellular proteins. At the end of the AHA incubation the cells were starved for methionine for $1 \mathrm{~h}$ and then pulsed briefly with HPG $(3 \mathrm{~h})$. This ensures that HPG, unlike AHA, is incorporated only into the proteins that were most recently synthesized. These analogues were coupled to two different fluorescent dyes through sequential click reactions. This was followed by imaging of the two fluorophores using two-color STED microscopy (Figure 3-6A) and overlaying averaged line scans obtained from the spots for each channel (Figure 3-6B, see Section 2.19 for details of the data analyses). Both amino acids localized to the same clouds (Figure 3-6A), although the new, recently synthesized proteins (revealed by HPG) did not enrich to the same extent in the cloud centers. This suggests that not only proteins of different ages localize to the same protein clouds, but also that the cloud centers might have a lower turnover. This could be related to a lower penetrance of proteins into the dense cloud centers, which would lead to a lower exchange.

Here one should note that the fold enrichment of the proteins over the baseline is an underestimate, since the baseline region obtained by this measurement (lateral to the peak) includes signal from a considerable proportion of large protein clouds, whose cloud areas overlapped with the baseline bordering the more frequent small clouds (see size distribution in Figure 3-3D). There is also a resolution difference between the two STED channels, for red channel it is $\sim 40-50 \mathrm{~nm}$ and for green $\sim 80 \mathrm{~nm}$. Therefore the underestimation is higher for the lower resolution green channel. When the general protein staining was imaged in the red channel as in Figure 3-3B, the peak brightness is 3 -fold over the baseline, which is considered to be closer to reality (compared to the $\sim 2$-fold protein enrichment obtained with the green channel for example in Figure 3-6A). 
A AHA - old proteins HPG - new proteins

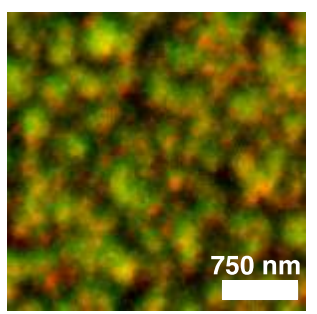

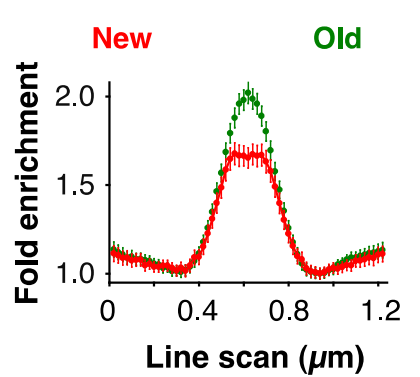

B

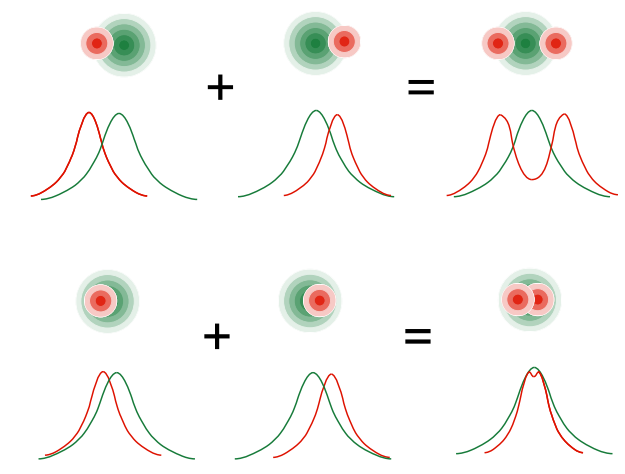

Figure 3-6 Two-color STED imaging of proteins of different ages.

A. Cells were subject to incubation with AHA for $72 \mathrm{~h}$ (old proteins, green), followed by HPG incubation for $3 \mathrm{~h}$ (new proteins, red). The relative distributions of the different labels were analyzed by performing line scans centered on the green AHA spots, as depicted in panel B. Note the co-localization of old and new proteins in the same spots (clouds). The graph indicates averaged line scans, normalized to the baseline, from 856 protein clouds (mean \pm SEM). B. The scheme indicates the general analysis procedure for 2-color STED images. Line scans were performed through protein clouds, and the signals corresponding to the red channel (various subsets of proteins) and green channel (protein cloud labeling) were obtained and averaged. If proteins in red channel are found predominantly at the edges of clouds, the average red line scan has two peaks. If they are randomly distributed along the clouds the average red line scan mimics that of general protein staining (green). If there is a preference for the center, the red curve is expected to be tighter than the green curve.

\subsection{Subsets of Proteins in the Clouds}

A similar sequential click-based procedure was performed to test the localization of proteins with different modifications, such as different lipid anchored proteins (palmitoyl, farnesyl, myristoyl, geranylgeranyl), or glycosylated proteins ( $\mathrm{N}$-acetylglucosamine, $\mathrm{N}$-acetylgalactosamine, $\mathrm{N}$ acetylmannosamine, fucose). I took advantage of the fact that click-able analogues for all of these protein modifiers are commercially available. This allows the investigation of the localization tendencies for proteins modified with different modifiers versus the localization of all other proteins, revealed as above by incorporation of amino acid analogues and two-color STED microscopy (Figure 3-7A-B). I have also investigated the distribution of a model glycophosphatidylinositol (GPI) anchored protein. For that, I have expressed the acyl carrier protein (ACP) with a GPI anchor signal sequence, and labeled it by coupling to Atto647N through the ACP-synthase reaction, Figure 3-7C). As indicated in Figure 3-7, each of the modified subsets of proteins overlapped well with the clouds. Occasionally distributions were not identical with those of general proteins. For example, the palmitoylated proteins were located especially in the cloud centers, forming a tighter distribution than general proteins (lateral spread 30\% smaller; 4 independent experiments), while farnesylated proteins showed a broader distribution (Figure 3-7A). 
A
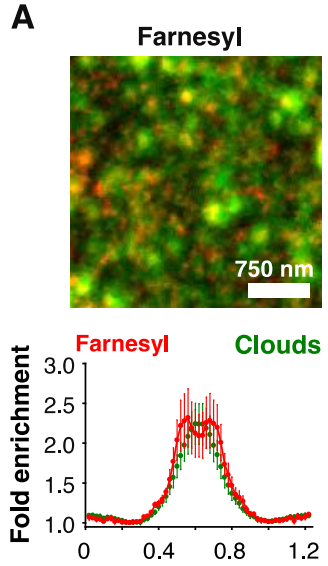

Geranylgeranyl

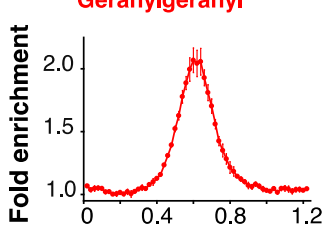

Palmitoyl

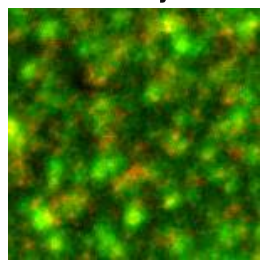

Palmitoyl
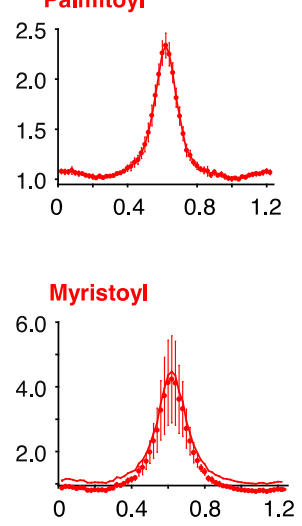

B

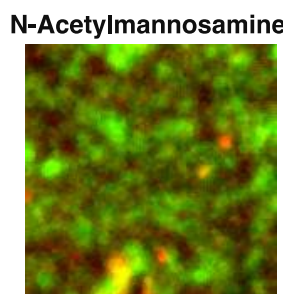

N-Acetylmannosamine

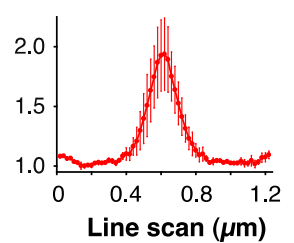

$\mathrm{N}$-Acetylgalactosamine

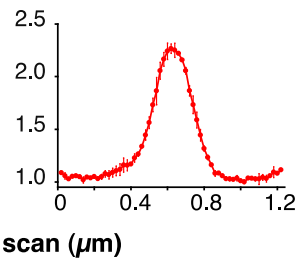

c

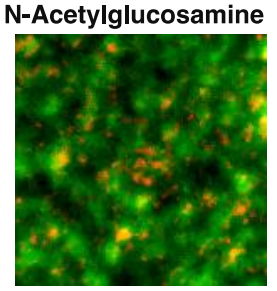

$\mathrm{N}$-Acetylglucosamine
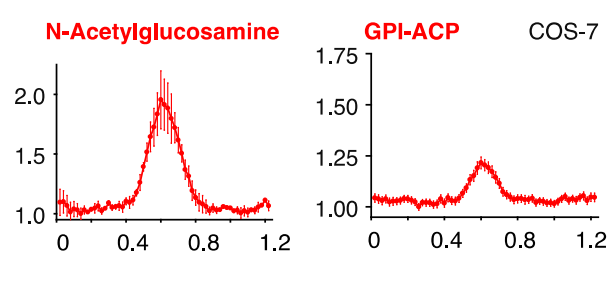

Figure 3-7 Different classes of proteins localize to the protein clouds.

A-B. Representative two-color STED microscopy images of membrane sheets click-labeled for several protein modifications (red) and for general protein clouds (green) sequentially. The graphs in the lower panels indicate averaged line scans, normalized to the baseline, $n=3-4$ independent experiments (mean \pm SEM) with a total of 629-1336 analyzed protein clouds. In parallel to metabolic labeling with methionine analogues, PC12 cells were also given clickable analogues of different lipid anchor modifiers, farnesyl, geranylgeranyl, palmitoyl and myristoyl (A) or glycosyl moieties, N-acetylmannosamine, galactosamine, glucosamine, and fucose (B) to label different classes of modified proteins. C. For GPI-anchor, a different procedure was used. A GPI-anchor for acyl carrier protein tagging was expressed in COS-7 cells and was coupled to Atto647N-CoA (red) through an ACP-synthase reaction before sonication of the cells and click labeling of protein clouds (green). Graph shows the average line scans, normalized to the baseline, from 600 protein clouds (mean \pm SEM).

\subsection{The Effects of Protein Clouds on Lipid Tendencies}

To investigate how lipids are organized with respect to the protein clouds, I have introduced fluorophore-coupled analogues for cholesterol, phosphatidylethanolamine (PE) and sphingomyelin (SM) into the membrane sheets of PC12 cells. The fluorescent lipid analogues are described in detail in Section 2.10.6 and Figure 2-2. In comparison to the distributions of proteins, the organization of lipids seemed to be almost independent of the clouds (Figure 3-8A). Cholesterol analogue was partially excluded from the cloud centers, while PE analogue, which is expected to be representative of glycerophospholipids in general, was virtually unaffected. The two chemically different SM analogues gave different distributions (discussed further in Section 4.3.3). In contrast, phosphotidylinositol-4,5-biphosphate (PIP2), detected by immunostaining (van den Bogaart et al., 2011), was found to be enriched mostly at the edges of protein clouds (Figure 3-8B). 
A

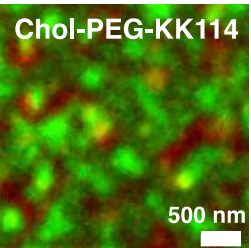

Clouds

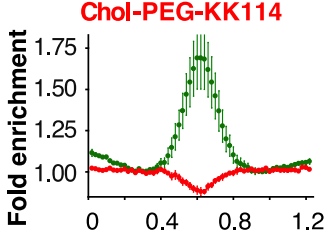

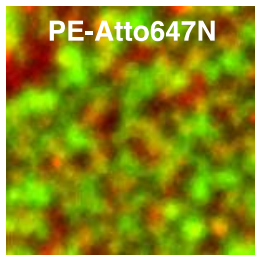

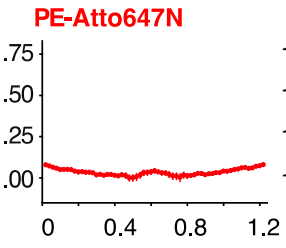

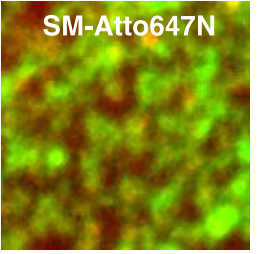

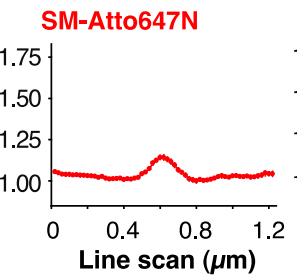

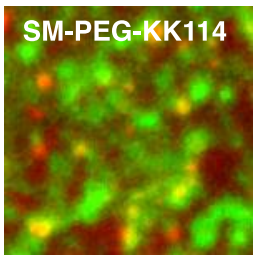

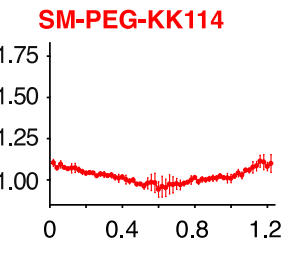

B
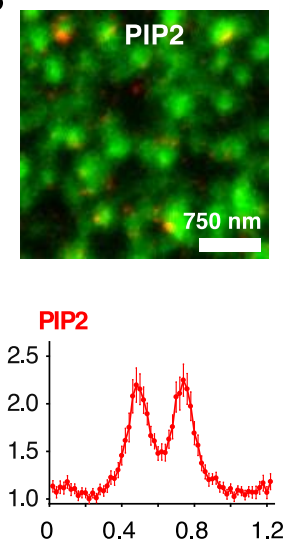

Figure 3-8 Lipid tendencies along the clouds.

A. Representative two-color STED microscopy images of PC12 membrane sheets click-labeled for general protein clouds (green) and were let to incorporate fluorescent lipid analogs, cholesterol-PEG-KK114 and PEAtto647N, SM-Atto647N and SM-PEG-KK114. The graphs in the lower panels indicate averaged line scans, normalized to the baseline, $n=3$ independent experiments (mean \pm SEM), with a total of 1370-1675 analyzed protein clouds. B. For probing PIP2, PC12 membrane sheets were immunostained with a primary PIP2 antibody, prior to fixation and click reaction, and detected by fluorophore-coupled secondary antibody (red). The graph shows averaged PIP2 line scan, normalized to the baseline, from 285 protein clouds (mean \pm SEM).

\subsection{The Influence of Protein Clouds on Diffusion}

After characterization of the clouds relative to subsets of proteins and lipids, I have moved on to determine whether evidence for the membrane patterning observed after click-chemistry could be reproduced in living, non-modified whole cells. By exploiting the fact that $\mathrm{N}$-acetylglucosamine and $\mathrm{N}$-acetylmanosamine moieties gave a very similar profile to that of protein clouds, I have used a lectin, wheat germ agglutinin (WGA), that binds to $\mathrm{N}$-acetylglucosamine and sialic acid residues on cellular membranes (Chazotte, 2011). WGA was coupled to the far-red fluorophore Star635 for STED imaging. Expectedly, WGA-Star635 labeling yielded a similar correlation with protein clouds in membrane sheets (Figure 3-9A). Therefore WGA was used as a marker for the protein clouds in living cells. To minimize endocytosis, the incubation time with WGA was kept as short as possible ( $60 \mathrm{~s})$. Flat regions of the upper cell membrane were imaged immediately after staining, using relatively flat cells such as COS-7 (Figure 3-9). STED images of the live-cell WGA staining showed a heterogeneous distribution of the marker (Figure 3-9B), in a pattern comparable to the protein clouds observed with click chemistry, and which appeared to be relatively stable over the imaging time.

To determine the behavior of proteins and lipids in relation to the protein domains identified by WGA, it was necessary to measure the diffusion in areas where the WGA is found in high 
abundance (indicative of protein clouds) versus the low abundance areas. For that, fluorescence correlation spectroscopy in combination with STED (STED-FCS) was employed (in collaboration with Alf Honigmann, MPI-BPC). FCS can determine lateral diffusion coefficients by analyzing the fluorescence intensity fluctuations caused by molecules diffusing randomly in and out of the excitation spot. In conventional FCS the size of the observation spot is limited by diffraction, which prevents the direct discrimination of heterogeneous dynamics occurring on scales below $200 \mathrm{~nm}$. However, by combining FCS with STED microscopy the observation spot can be decreased far below the diffraction barrier (Eggeling et al., 2009), thus improving the precision of the measurement.

For protein diffusion WGA-Star635 mobility was measured with respect to WGA-Star635 signal intensity (example image in Figure 3-9B). For lipid diffusion, the mobility of fluorescently labeled cholesterol analogue Chol-PEG-KK114 was measured with respect to the protein clouds probed by WGA-Alexa488 (example images in Figure 3-9C). The FCS observation spot was placed on 100 random positions on the plasma membrane of cells, and $10 \mathrm{~s}$ correlation measurements were performed (Figure 3-9D). For each of these measurements two parameters were obtained: the average lateral diffusion coefficient and the average intensity of the WGA staining at the respective positions (see Section 2.17 for more details about the measurements and the analyses). First, the cells were incubated with WGA-Star635 and its mobility was measured by STED-FCS in relation to the intensity of the WGA-Star635 spots (Figure 3-9E). In independent experiments, the cells were incubated with WGA-Alexa488 (as a probe for protein clouds) and with Chol-PEG-KK114, and the mobility of the cholesterol analogue was measured in relation to the WGA-Alexa488 staining at the different positions (Figure 3-9F). The diffusion coefficients of both WGA and the fluorescent cholesterol analogue were inversely correlated with the intensity of WGA staining, suggesting that both molecules diffuse more slowly within the protein clouds. 
A
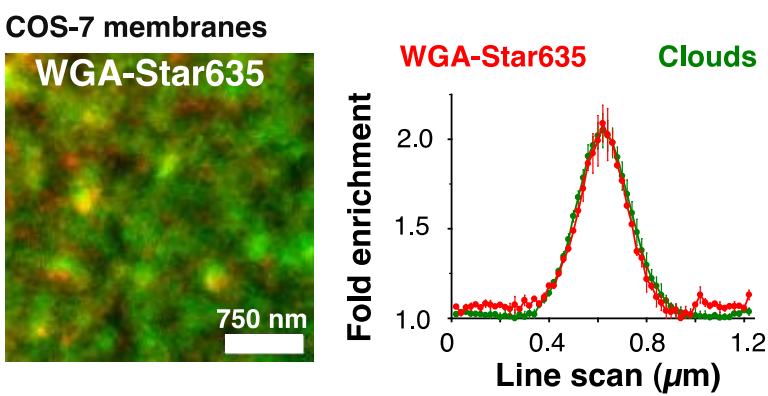

B

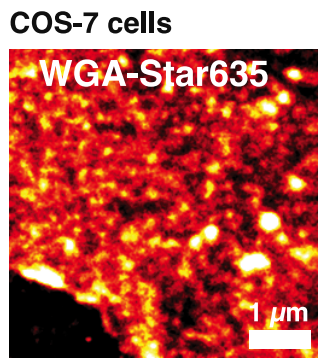

C

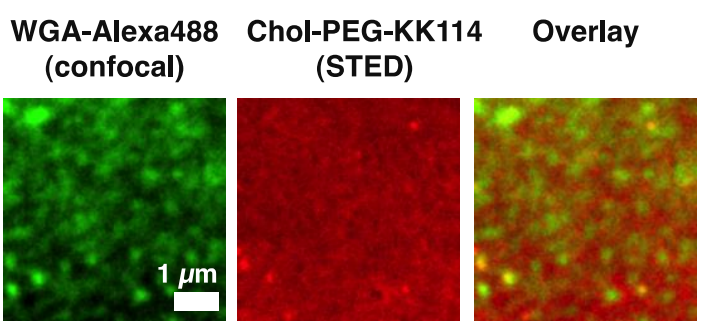

E

WGA-Star635

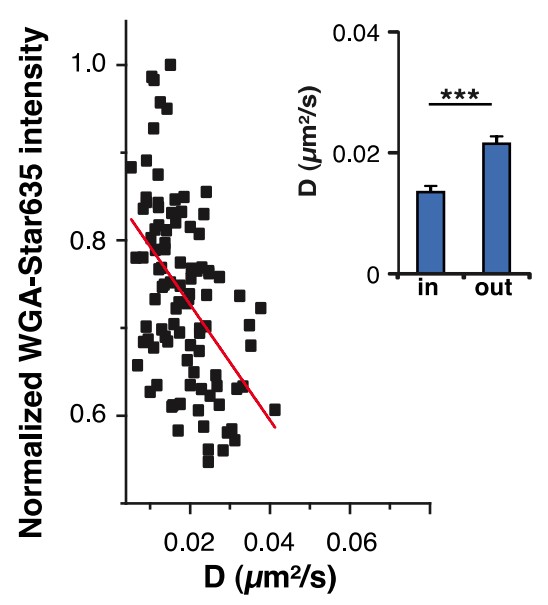

$\mathbf{F}$

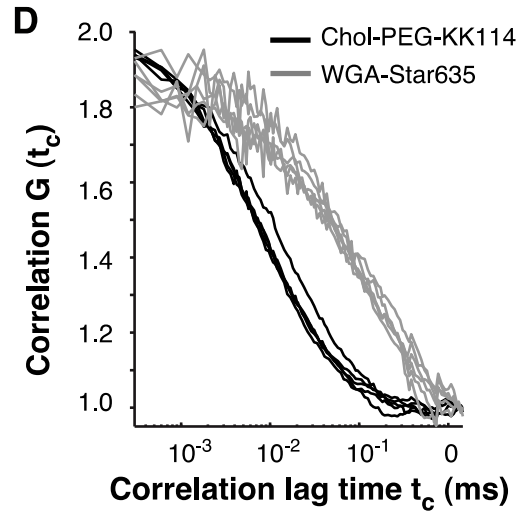

Chol-PEG-KK114

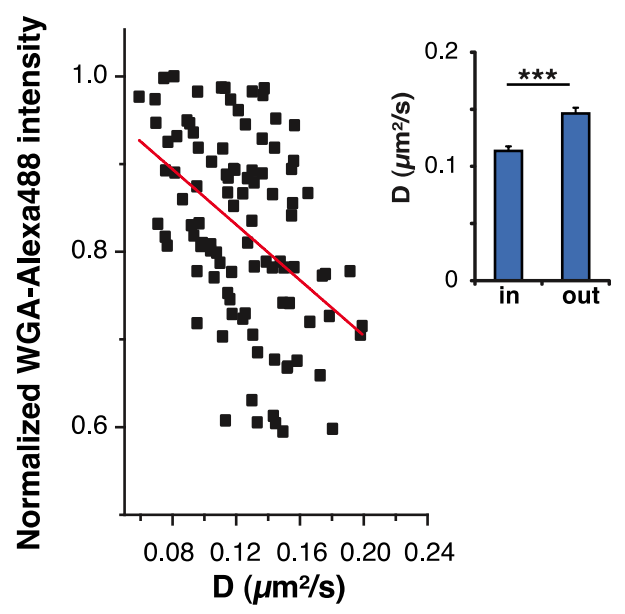

Figure 3-9 Mobilities of the cholesterol analogue and WGA are slower in the protein clouds.

A. Representative two-color STED microscopy images of COS-7 membrane sheets labeled with WGAStar635 (red) and for general protein clouds (green). COS-7 cells were incubated with WGA-Star635, were washed, fixed with $4 \%$ PFA, and click-labeled using Chromeo494 (green). The graph shows the averaged line scan, normalized to the baseline, from 410 protein clouds (mean \pm SEM). B-F. The mobility of WGA and of fluorescent cholesterol analogue (Chol-PEG-KK114) in the plasma membrane of live COS-7 cells was determined using STED-FCS. B. STED image of red fluorescent WGA-Star635 in living COS-7 cells. C. Twocolor staining of the plasma membrane of live COS-7 cells with Chol-PEG-KK114 (red, STED image) and WGA-Alexa488 (green, confocal image). D. Representative fluorescence correlation curves obtained from WGA-Star635 and Chol-PEG-KK114 at different positions on the upper membrane of live cells. E. Scatter plot of lateral diffusion coefficients of WGA-Star635, against the local normalized intensity in the membrane (100 data points, 5 independent samples). The diffusion coefficients are correlated with the normalized brightness. The red line shows a linear fit of the data, as a guide for the eye. F. Same as for panel E, for fluorescent cholesterol (Chol-PEG-KK114). The protein clouds were stained with WGA-Alexa488. The insets in $E$ and $F$ show the average diffusion coefficient in and out of the WGA clusters, indicating that diffusion was significantly slower inside the clusters both for WGA itself and also cholesterol. For the in and out of cluster (cloud) classification see Section 2.17 and Figure 2-3. ${ }^{* * *}, \mathrm{P}<0.001$, t-tests. The STED-FCS measurements and analyses were performed by Alf Honigmann. 
These results indicate that the diffusion of glycosylated proteins and lipids targeted by WGA is restricted inside the protein clouds, most probably by specific protein interactions and indirectly by molecular crowding. Interestingly, cholesterol diffusion was also decreased in the protein clouds, albeit to a lesser extent than for WGA. This may, in principle, be a result of specific interactions between cholesterol and proteins in the clouds. However, because the cholesterol analogue was slightly excluded from the center of the protein clouds in the membrane sheets (Figure 3-8A), the decrease in diffusion can be attributed to a passive effect induced by molecular crowding: the protein to lipid ratio is higher in the clouds, allowing less space for lipids and cholesterol to diffuse freely. Here, one should note that, although WGA was instrumental in labeling different groups of proteins in a manner technically compatible with live cell STED-FCS measurements, it is not the ideal probe due to its polyvalency in ligand binding (Monsigny et al., 2000). Therefore, it would be beneficial to reproduce these STED-FCS measurements with a monovalent probe to make sure that a potential clustering due to WGA does not affect the results.

\subsection{The Effect of Increasing the Amount of Protein at the Membrane}

It was shown in Figure 3-6 that proteins of different ages are co-localized in the same clouds. I wanted to know what happens when more proteins are targeted to the membrane. Could the clouds accommodate more proteins? To answer this question two abundant proteins of PC12 cells, SNAP25 (Figure 3-10A) and syntaxin 1 (Figure 3-10B), were overexpressed and sheets were obtained as in Figure 3-3. The overexpression did not affect the overall membrane pattern, but it was observed that the relative intensity of the low-abundance areas was slightly but significantly higher in the overexpression cases (Figure 3-10C). This suggests that some of the overexpressed proteins spilled over into these areas - possibly due to the clouds only being able to accommodate a certain density of proteins. This experiment also confirms that the sensitivity of our labeling and detection methods yields a high enough contrast to detect subtle variations arising from altered levels or distributions of specific proteins. 

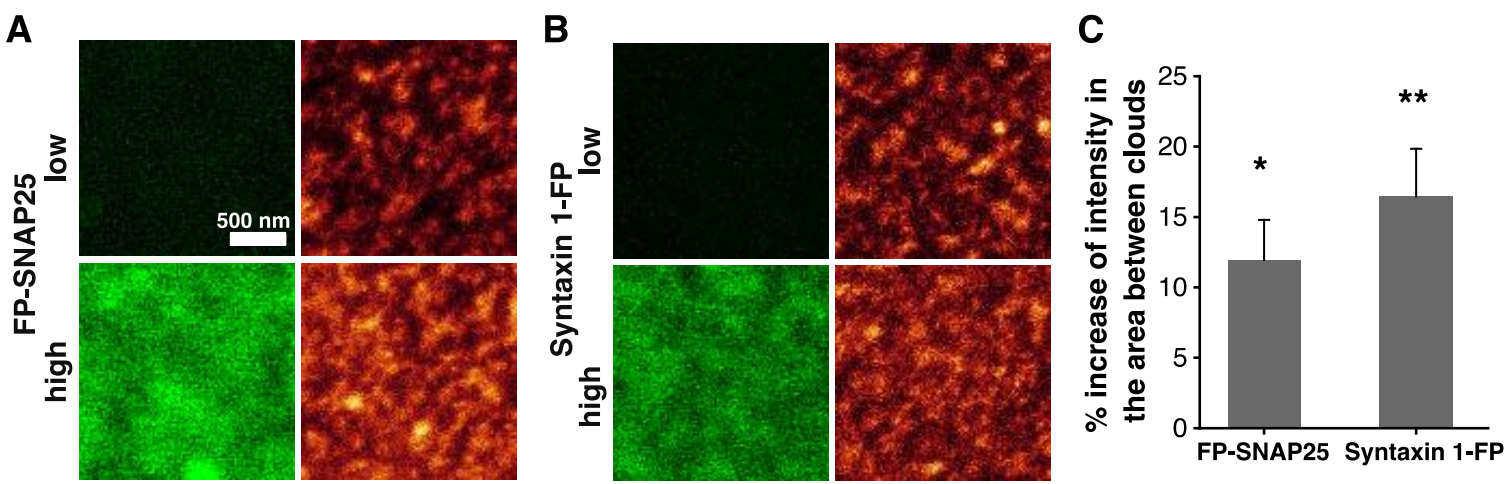

Figure 3-10 Effects of protein overexpression on the general protein organization.

A-B. Fluorescently tagged versions of SNAP25 (A) and syntaxin 1 (B) were overexpressed in PC12 cells. The left column displays the fluorescent protein (FP) signal (confocal images). The protein distribution is shown on the right (STED images). Image intensities are scaled identically. High: membranes expressing high levels of FP-SNAP25 or syntaxin 1-FP, respectively. Low: membranes expressing low levels of the exogeneous proteins. C. The graph quantifies the increase in fluorescent intensity in the area between clouds as percentage of the non-overexpressing control membranes. The graph indicates mean $\pm \mathrm{SEM}$; $n=18-37$ sheets per condition, $\mathrm{P}=0.021\left(^{*}, \mathrm{P}<0.05\right)$ for SNAP25 and $\mathrm{P}=0.0047$ for syntaxin $1(* *, \mathrm{P}<0.01)$, t-tests.

\subsection{The Factors That Are Involved in Cloud Organization}

\subsubsection{The Stability of the Clouds under Different Conditions}

The observations presented so far suggest that protein clouds are stable and relatively long-lived formations, as they appear to persist over time scales from seconds (Figure 3-9) to hours (Figure 3-6), which can fit a certain density of proteins (Figure 3-10). To test their stability further and to dissect the factors involved in their organization, membrane sheets were subject to many harsh treatments proposed to influence the patterning of many subgroups of membrane proteins: strong increases or decreases in ionic strength, abrupt changes in $\mathrm{Ca}^{2+}$ concentration (addition of $1 \mathrm{mM} \mathrm{Ca}^{2+}$ or more physiological changes induced by ionomycin application; Figure 3-11A-B), reduction of protein crowding by knocking down abundant membrane proteins (syntaxin 1 and SNAP25; Figure 3-11B), or microtubule dispersion (Figure 3-11C). All of these treatments were unable to affect the overall area occupied by the protein clouds, indicating that they could not alter the forces grouping proteins within the clouds. This parameter also remained constant after one-hour treatments with actin-depolymerizing drugs such as latrunculin $A$ or cytochalasin $D$, or after removing sphingomyelin by applying the enzyme sphingomyelinase (SPMase, Figure 3-11C). The same observation applied to several other lipid-hydrolyzing enzymes, the phospholipases A2, $C$ and $D$ (Figure 3-11D). For each condition, the area quantification values were always normalized to their respective controls that were treated the same in way in terms of drug vehicle (DMSO or buffer), and incubation time and temperature after sonication. 

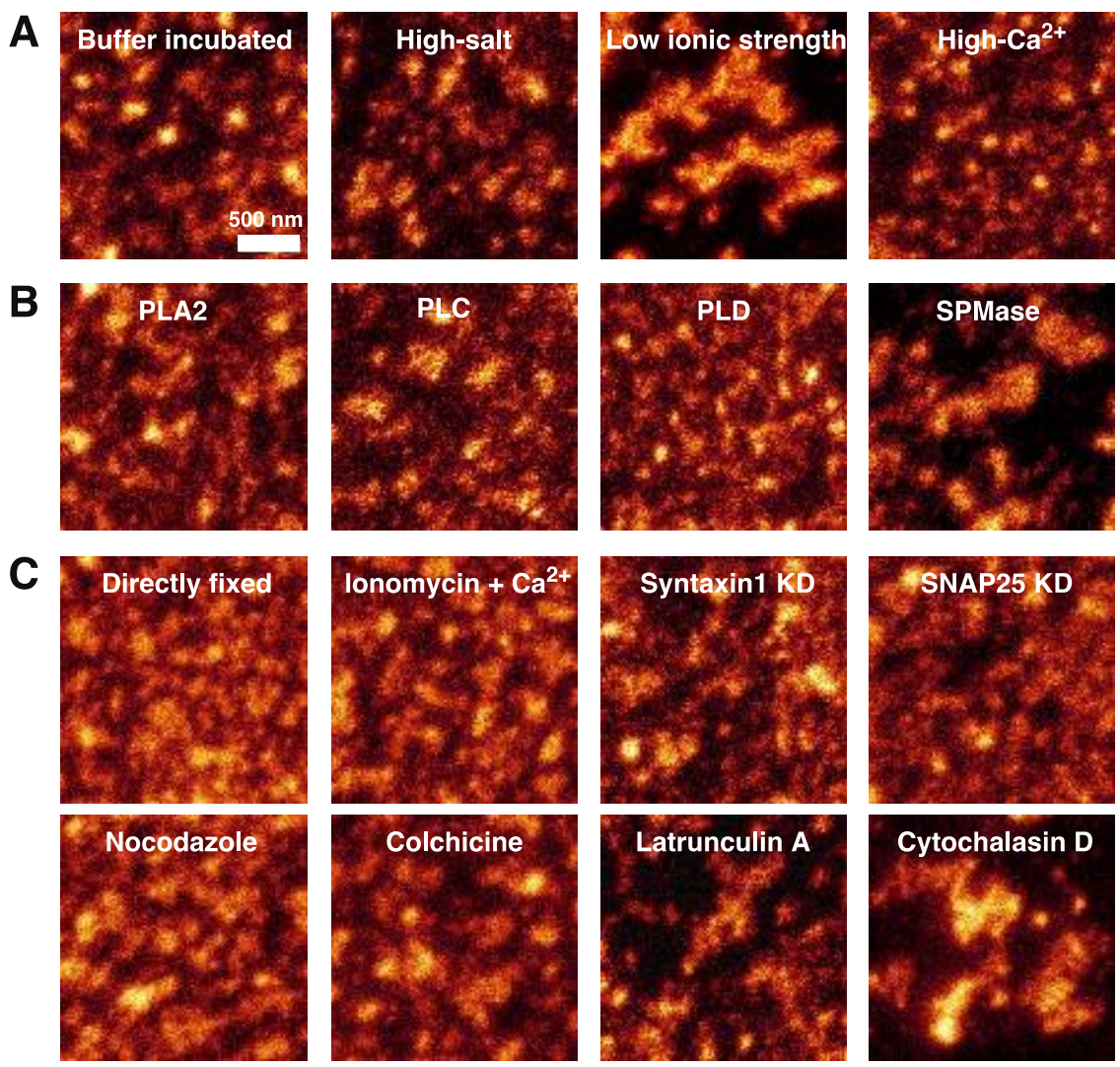

D

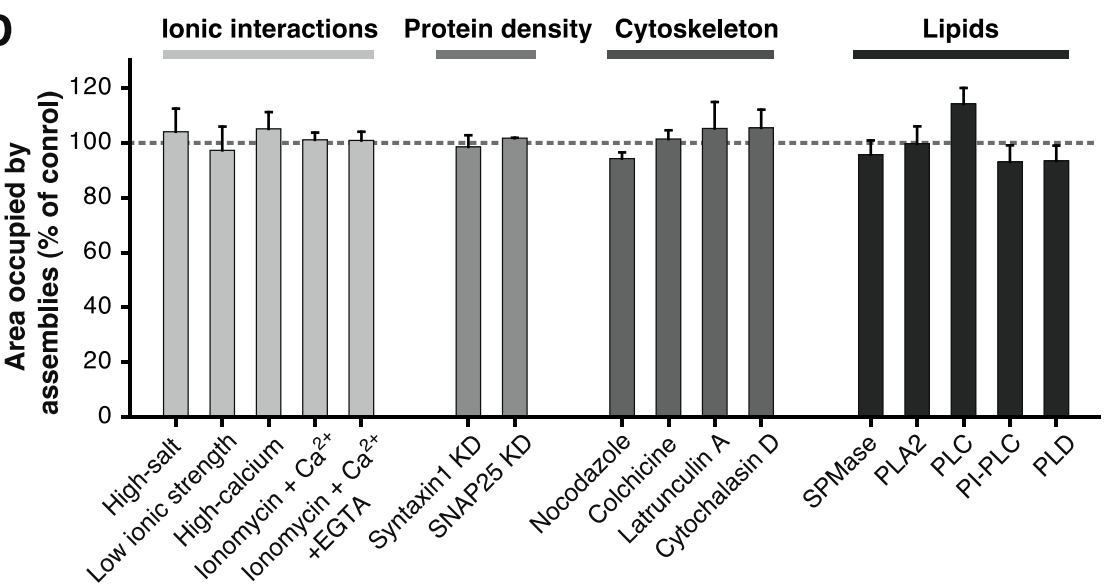

Figure 3-11 Protein cloud organization persists after many harsh treatments.

STED imaging of protein clouds in PC12 sheets subjected to different treatments either applied on sheets before fixation (A-B), or onto live cells before generation of sheets (C). A. Membrane sheets were either incubated in sonication buffer (leftmost image) as control or treated with high-salt buffer $(0.5 \mathrm{mM} \mathrm{KCl})$, low ionic strength buffer ( $320 \mathrm{mM}$ sucrose, $5 \mathrm{mM}$ HEPES-KOH) or $1 \mathrm{mM} \mathrm{Ca}^{2+}$ for 20 minutes at $37^{\circ} \mathrm{C}$. B. Lipid composition of the membranes was manipulated by applying 1-2 units/mL phospholipases (PL) A2, C, D and phosphatidylinositol (PI)-specific PLC (not shown) or 20 units $/ \mathrm{mL}$ sphingomyelinase (SPMase) diluted in sonication buffer, for $15 \mathrm{~min}$. C. For treatments on full cells, controls were prepared by chemically fixing the membrane sheets directly after sonication. For ionic perturbation, cells were treated for 5 min with $1 \mu \mathrm{M}$ of ionomycin in HEPES-buffered medium containing $5 \mathrm{mM} \mathrm{Ca}^{2+}$, with or without $10 \mathrm{mM} \mathrm{EGTA.} \mathrm{In} \mathrm{independent}$ experiments, the abundant membrane proteins syntaxin 1 or SNAP25 were knocked down by siRNAs. To manipulate the cytoskeletal elements, cells were treated for $1 \mathrm{~h}$ with $0.1 \%$ DMSO (as solvent control, not shown), $10 \mu \mathrm{M}$ nocodazole, colchicine, or cytochalasin D, or $1 \mu \mathrm{M}$ latrunculin A. D. For all the conditions, the area occupied by the clouds was quantified and expressed as \% of the respective control for each condition (see Section 2.19 for details). $n=3-5$ independent experiments, means \pm SEM for all treatments, except for the knockdown (KD) conditions, where means \pm range of values from 2 independent experiments are shown. $\mathrm{P}>0.05$ for all conditions, $\mathrm{t}$-tests. 
After these harsh treatments, although the overall area covered by protein clouds was not strongly affected, several of them had a visible effect; the clouds looked larger and more distant from each other. The cloud size was quantified to understand the extent of this effect as shown in Figure 3-12.

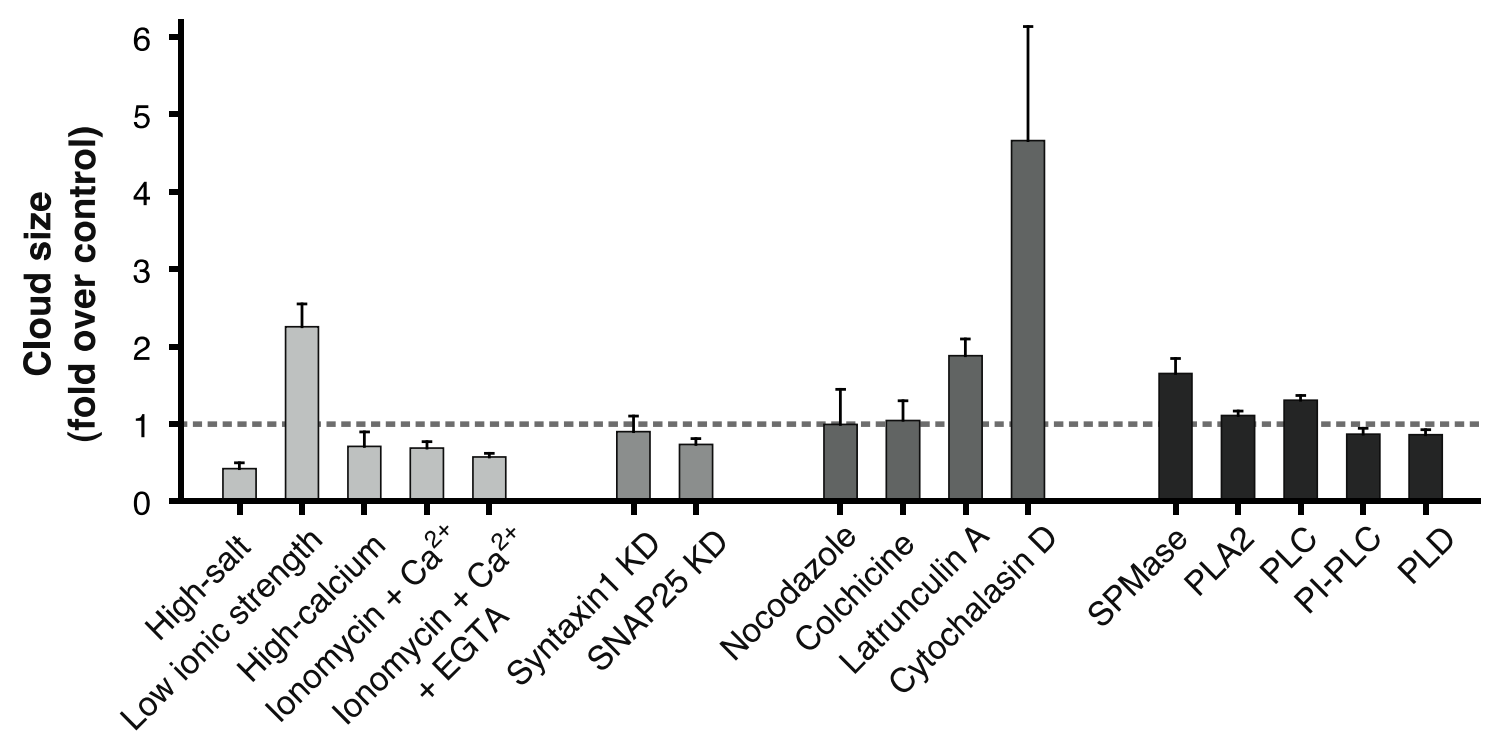

Figure 3-12 Disruption of actin results in larger clouds.

The graph indicates the cloud sizes (as fold increase over the control conditions) for the different treatments investigated (mean \pm SEM, from 3-5 independent experiments for all conditions except SNAP25 and syntaxin $1 \mathrm{KD}$, for which the range of values is shown from 2 independent experiments. The strongest phenotype was obtained upon reducing ionic strength, as membrane clouds appeared to coalesce, although the general membrane coverage was unaffected.

\subsubsection{Actin}

The conditions that created larger clouds were low ionic strength buffer, latrunculin A, cytochalasin D and SPMase. Latrunculin A and cytochalasin D point to an actin-related phenotype. After treatment with these actin-depolymerizing drugs, although the membrane still had substantial actin coverage (Figure 3-13A,C), actin filaments were substantially lost (Figure 3-13B). Interestingly, low ionic strength buffer and SPMase also had an effect on the actin cytoskeleton, not only the filaments but also the general actin staining on the membrane was substantially lost under these conditions (Figure 3-13C). Here one should note that the effect of SPMase is expected to be a combination of the direct sphingomyelin removal action and indirect side-action (Zeidan et al., 2008) that causes massive actin loss. It can also be seen that in terms of formation of larger clouds losing the filaments is more effective than losing the general actin staining (Figure 3-13B-C), suggesting that different populations of actin molecules might be serving for different purposes in organization. 
A

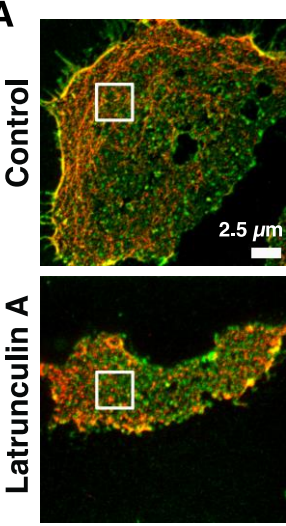

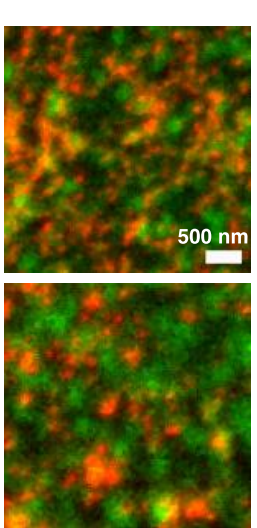

B

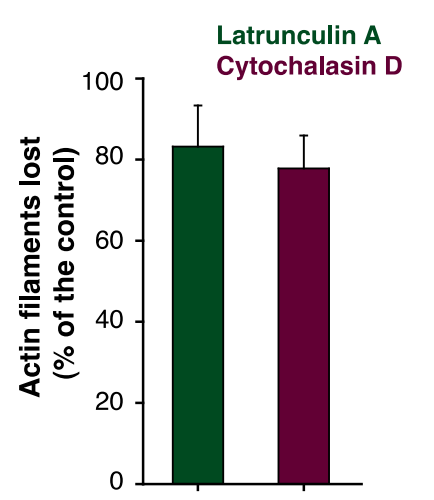

C

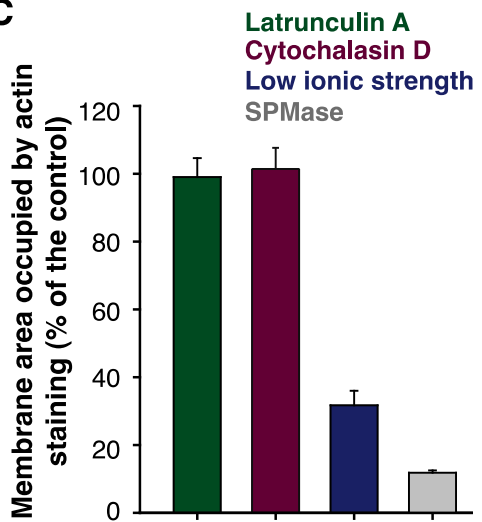

Figure 3-13 Quantification of the actin disruption

A. After $1 \mathrm{~h}$ treatment with $1 \mu \mathrm{M}$ latrunculin $\mathrm{A}$, there was still a substantial level of actin staining on the membrane, as revealed by immunostaining of the click-labeled PC12 sheets for actin and imaging with 2color STED. B. After latrunculin A or cytochalasin D treatments, majority of the fibrils were lost in comparison to the control condition (mean \pm SEM, 3 independent experiments) C. The graph shows total actin fluorescence (obtained from confocal images) for different treatments as percentage of the respective control (mean \pm range of values, 2 independent experiments). The incubations were performed as described for Figure 3-11.

These observations suggest that the membrane-associated actin cytoskeleton is important for maintaining the protein clouds apart from each other or for limiting their size. However, actin does not appear to be essential for the existence of cloud-like formations.

\subsubsection{Cholesterol}

A much stronger effect on the membrane pattern was observed when cholesterol was depleted from membrane sheets. Surprisingly, the entire cloud organization was eliminated by the removal of cholesterol via methyl- $\beta$-cyclodextrin (MBCD) (Figure 3-14). The simplest interpretation of this phenomenon is that proteins diffused out of the clouds, resulting in a much more homogeneous distribution. Small fluorescent puncta $(<60 \mathrm{~nm})$ were still occasionally visible, suggesting that some protein clusters survive this treatment. These are probably stabilized by cholesterolindependent interactions, such as specific protein-protein binding or still hold in place by residual levels of cholesterol. The effects of MBCD were mimicked by cholesterol oxidase (COase) and by a chemically different $\beta$-cyclodextrin, but not by the structurally related $\alpha$-cyclodextrin (Figure 3-14B), which is unable to extract cholesterol (Ohtani et al., 1989; Zidovetzki, 2007). Importantly, cholesterol replenishment after MBCD treatment (Zidovetzki, 2007) recovered the membrane patterning (Figure 3-14), suggesting that the cholesterol influence is very strong. Actin was still present after cholesterol depletion (membrane coverage of $108.3 \pm 10.7 \%$ of the control), indicating that the cholesterol depletion effects are not directly connected to actin. 
A

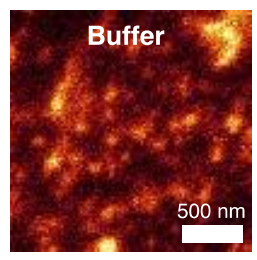

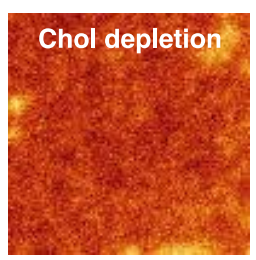

B

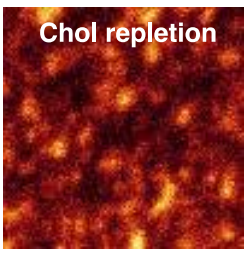

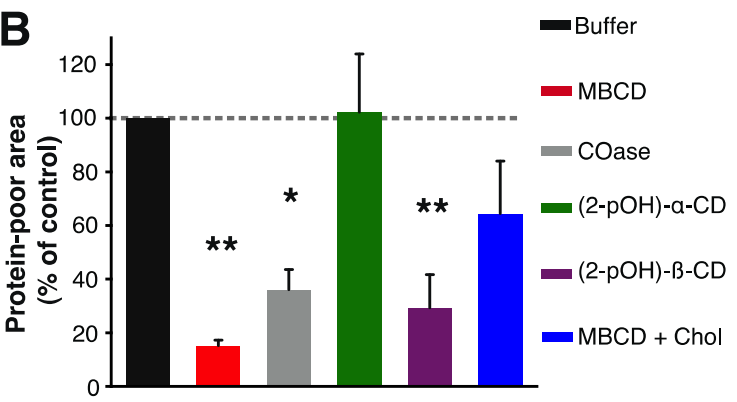

Figure 3-14 Cholesterol controls the cloud formation

A. The panels show STED images of membrane sheets treated with sonication buffer ( 15 minutes at $37^{\circ} \mathrm{C}$, left), with MBCD ( $5 \mathrm{mM}, 5$ minutes followed by incubation in sonication buffer for 10 minutes at $37{ }^{\circ} \mathrm{C}$, middle), and with $\mathrm{MBCD}$ ( $5 \mathrm{M}, 5$ minutes) followed by $\mathrm{MBCD}$-cholesterol (to replete cholesterol, $5 \mathrm{mM}, 10$ minutes, at $37{ }^{\circ} \mathrm{C}$, right). B. The effect was quantified by finding the area of the protein-poor regions as convenient measure for the loss of protein clouds. This protein-poor fraction is predicted to be 0 upon the complete dispersion of proteins from the clouds. All values were expressed as \% of control (buffer incubation alone). The bars show the following treatments: $M B C D$ treatment as in the middle panel above, 0.2 units/ml COase for 15 minutes at $37{ }^{\circ} \mathrm{C}, 25 \mathrm{mM}$ alpha or beta-hydroxypropyl-CD for 5 minutes at $37^{\circ} \mathrm{C}$, cholesterol replenishment as in the left panel above. $\mathrm{n}=3-7$ independent experiments $(*, \mathrm{P}<0.05 ; * *, \mathrm{P}<$ 0.01 ; t-tests). Note the loss of protein-free areas upon cholesterol depletion, and their re-formation (along with protein clouds) upon cholesterol repletion.

I have also verified the importance of cholesterol in other cellular membranes. I obtained different organelles from AHA-treated PC12 cells, fixed them and subjected them to click reactions. Just as in the plasma membrane sheets, proteins were clustered in the membranes of early endosomes, and cholesterol depletion caused their dispersion (Figure 3-15A, C). On the contrary, mitochondrial membranes, which contain 40 -fold lower levels of cholesterol than the plasma membrane (Daum, 1985), were dominated by a homogeneous protein distribution. Loading them with cholesterol induced the formation of clusters (Figure 3-15B, C). This suggests that cholesterol is a major membrane-organizing molecule, able to act even in membranes where it is not normally present at high levels. 


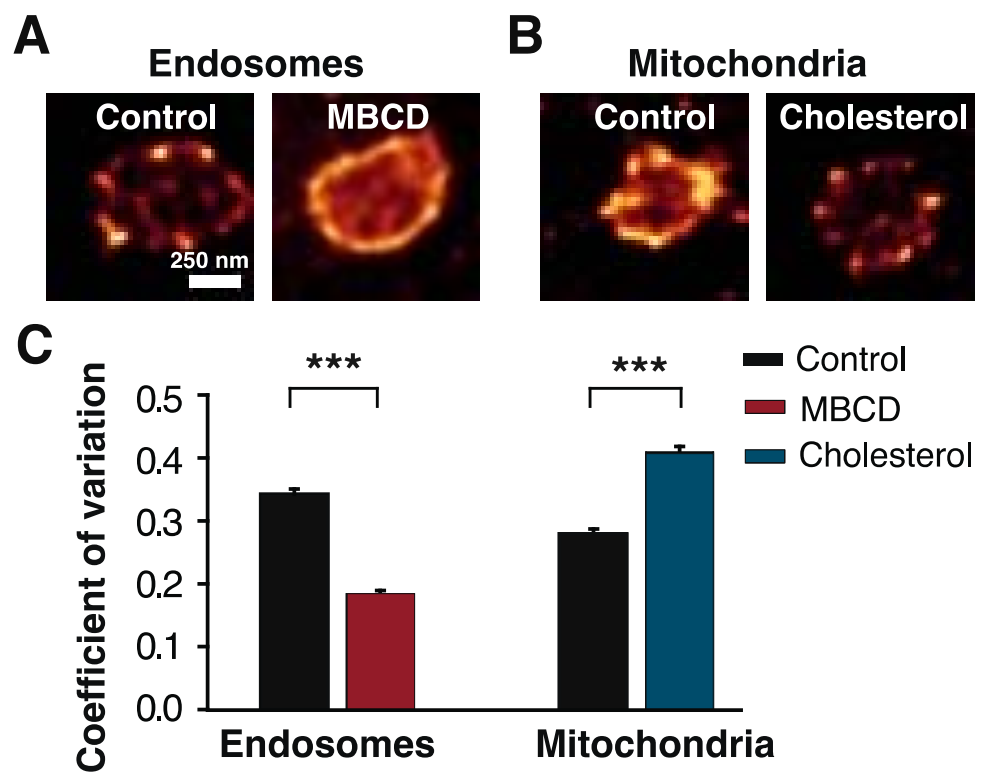

Figure 3-15 Effect of cholesterol on clouds can be reproduced on organellar membranes

A. STED images of a normal (control) early endosome and of an early endosome treated with $45 \mathrm{mM} \mathrm{MBCD}$ for 30 minutes at $37^{\circ} \mathrm{C}$. B. STED images of a control mitochondrion and of a mitochondrion after cholesterol addition (from $5 \mathrm{mM}$ cholesterol-MBCD complexes for 30 minutes at $37^{\circ} \mathrm{C}$ ). In both panels $\mathrm{A}$ and $\mathrm{B}$, the organelles were obtained from AHA-treated PC12 cells and treated in vitro. The images are processed by deconvolution for display purposes. C. The graph shows the quantification of the clustering of membrane proteins in endosomes and mitochondria; the coefficient of variation was measured along the membrane as an indication of the level of clustering. $n>110$ organelles per condition, from multiple independent experiments (***, $\mathrm{P}<0.001$, t-tests).

The effects of cholesterol depletion and repletion as well as the effects of ionic conditions were also reproduced in COS-7 membrane sheets (Figure 3-16). Again, relatively minor effects were seen with most ionic treatments, but larger clouds were observed under low ionic strength condition (actin phenotype) and cloud organization was lost under cholesterol depletion. Cholesterol repletion recovered the protein clouds. 

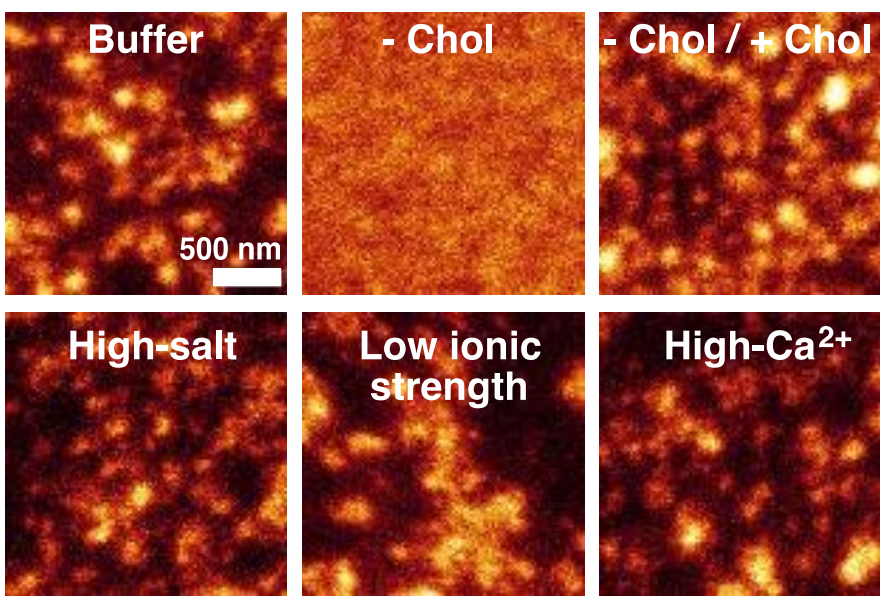

Figure 3-16 Similar phenotypes observed in COS-7 cells.

The panels show STED images of membrane sheets from COS-7 cells treated as in Figure 3-11. Briefly, the sheets were treated with buffer only (15 minutes), MBCD (5 mM, 5 minutes), with MBCD followed by MBCD-cholesterol (to replete cholesterol, $5 \mathrm{mM}, 10$ minutes), with high-salt buffer $(\mathrm{KCl}, 0.5 \mathrm{mM}, 20$ minutes), with low ionic-strength buffer ( $320 \mathrm{mM}$ sucrose, $5 \mathrm{mM} \mathrm{HEPES}-\mathrm{KOH}, 20$ minutes) or with high $\mathrm{Ca}^{2+}$ ( $1 \mathrm{mM}, 20$ minutes). Note that the phenotypes are similar to those observed in PC12 sheets.

The effects of cholesterol depletion were also verified in living cells, by pre-treating the cells with COase (Eggeling et al., 2009), before STED-FCS measurements (performed as in Figure 3-9). WGA staining after the treatment still showed a heterogeneous distribution, but with reduced contrast between bright and dark regions. While in control cells both WGA and the fluorescent cholesterol analogue were diffusing more slowly within the protein clouds (Figure 3-9), this difference was no longer observed after COase treatment (Figure 3-17A-B). Diffusion coefficients were higher in COase-treated cells, for both WGA and cholesterol (1.7 and 1.3-fold, respectively), and were similar inside or outside the protein clouds (Figure 3-17A-B). This is also underlined by calculating the Pearson's correlation coefficient between the WGA cloud intensity and the diffusion coefficient (Figure 3-17C). While in control cells a strong negative correlation is seen, caused by the decreased diffusion within protein clouds, this effect is much less evident after COase treatment.

Just as for membrane sheets, these results indicate that the integrity of protein clouds depends on cholesterol in living cells. Reducing cholesterol levels leads to a dispersion of proteins in the membrane, causing higher diffusion rates. The fact that the domains did not completely disperse after COase treatment is most likely due to residual cholesterol levels in the plasma membrane of living cells. Depletion in living cells is not as efficient as in the sheets, since only the cholesterol molecules on the outer leaflet can be attacked and the cells can adapt by targeting more cholesterol to the membrane. 
A cos-7 cells

WGA-Star635

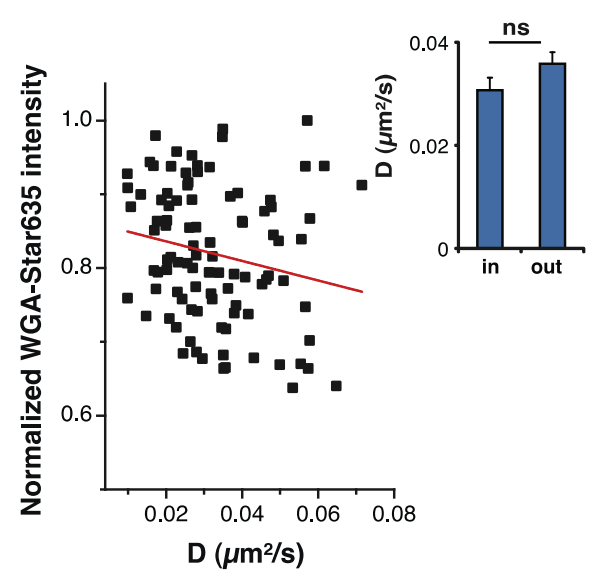

B

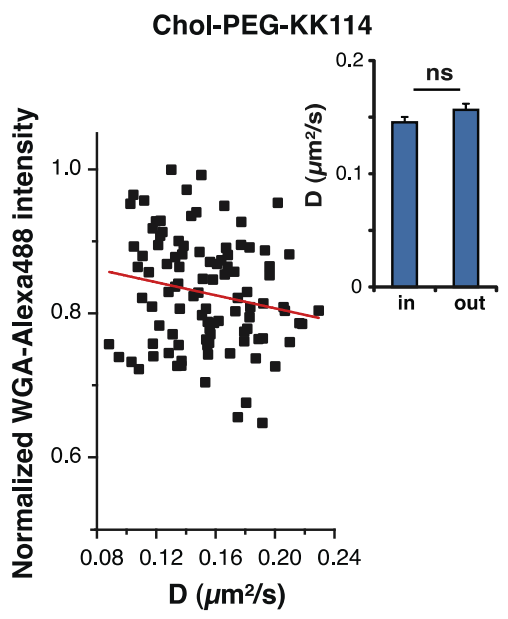

C

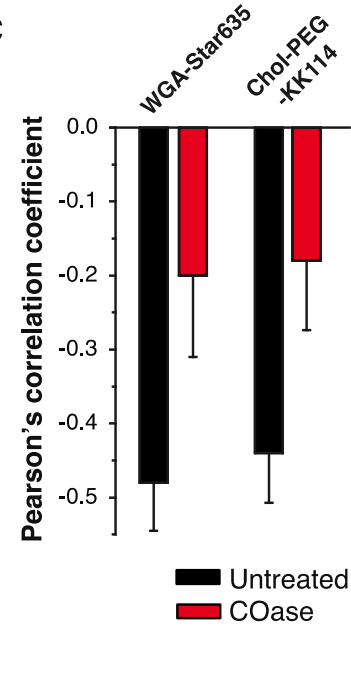

Figure 3-17 STED-FCS measurements on living cells treated with COase.

A. Scatter plot of diffusion coefficients of WGA-Star635, displayed against the local normalized intensity in the membrane (presented as in Figure 3-9. B. Same as A, for fluorescent cholesterol (Chol-PEG-KK114). Cholesterol reduction increased the mobility of both WGA and fluorescent cholesterol (compare with Figure 3-9; increases of 1.7 fold and 1.3 fold, for WGA and cholesterol, respectively). The insets in panels A and B show that diffusion inside and outside of the WGA clusters was not significantly different after cholesterol reduction. $\mathbf{C}$. The correlation between diffusion and the brightness of protein clouds was strongly reduced (Pearson correlation coefficient, PCC, values of approximately - 0.2 , as opposed to approximately -0.5 in controls. The graph indicates mean \pm standard deviation from a bootstrap analysis. The measurements and analyses were performed by Alf Honigmann.

\subsection{Distributions of Specific Proteins within the Clouds}

To investigate the relevance of the protein clouds for specific proteins, I probed several integral and peripheral proteins (listed in Table 3-1) at the membrane by immunostaining membrane sheets, in addition to the click labeling of protein clouds. The proteins included transmembrane, lipid-anchored and peripheral membrane proteins, as well as cytoskeletal proteins. Different functional protein classes were targeted, including proteins involved in neurotransmitter release (exocytosis), constitutive secretion, membrane recycling and cytoskeletal modifications (Table 3-1). The STED images indicate that all these proteins co-localize with the clouds to a good extent but are enriched in different positions relative to cloud centers (Figure 3-18). Most proteins were only present in some subsets of the clouds, indicating that not all clouds are the same, as one would expect. 
Table 3-1 Summary of all specific proteins investigated.

\begin{tabular}{|c|c|c|c|c|c|c|}
\hline Protein name & $\begin{array}{l}\text { Membrane } \\
\text { association }\end{array}$ & $\begin{array}{c}\text { Post- } \\
\text { translational } \\
\text { anchor }\end{array}$ & Localization & $\begin{array}{l}\text { General } \\
\text { function }\end{array}$ & $\begin{array}{l}\text { Enrich } \\
\text {-ment } \\
\text { in } \\
\text { clouds }\end{array}$ & $\begin{array}{l}\text { Center- } \\
\text { ness }\end{array}$ \\
\hline Actin & Cytoskeletal & & $\begin{array}{l}\text { Cytoplasm / } \\
\text { cell cortex }\end{array}$ & $\begin{array}{c}\text { Cytoskeletal } \\
\text { element }\end{array}$ & 1.77 & 0.49 \\
\hline APP & $\begin{array}{l}\text { Bitopic } \\
\text { Integral }\end{array}$ & $\begin{array}{c}\text { Palmitoylation } \\
\text { (2 sites, rarely } \\
\text { happens) } \\
\text { (Bhattacharyy } \\
\text { a et al., 2013) }\end{array}$ & $\begin{array}{c}\text { Endomembra } \\
\text { ne system }\end{array}$ & $\begin{array}{c}\text { Cell surface } \\
\text { receptor of } \\
\text { neurons }\end{array}$ & 4.31 & 1.51 \\
\hline BACE & $\begin{array}{l}\text { Bitopic } \\
\text { Integral }\end{array}$ & $\begin{array}{l}\text { Palmitoylation } \\
\text { (4 sites) } \\
\text { (Vetrivel et al., } \\
\text { 2009) }\end{array}$ & $\begin{array}{c}\text { Endomembra } \\
\text { ne system }\end{array}$ & $\begin{array}{l}\text { Proteolytic } \\
\text { processing of } \\
\text { the amyloid } \\
\text { precursor } \\
\text { protein (APP) }\end{array}$ & 4.00 & 0.69 \\
\hline Bassoon & Peripheral & $\begin{array}{c}\mathrm{N} \text {-terminal } \\
\text { myristoylation }\end{array}$ & $\begin{array}{l}\text { Cytoplasm / } \\
\text { transport } \\
\text { vesicles / cell } \\
\text { membrane }\end{array}$ & $\begin{array}{l}\text { Organization of } \\
\text { the cytomatrix } \\
\text { at the active } \\
\text { zone }\end{array}$ & 2.35 & 0.51 \\
\hline$\alpha-N$-catenin & Peripheral & & $\begin{array}{c}\text { Cell } \\
\text { membrane / } \\
\text { cytoplasm }\end{array}$ & $\begin{array}{l}\text { Linker between } \\
\text { cadherin } \\
\text { adhesion } \\
\text { receptors and } \\
\text { the } \\
\text { cytoskeleton, to } \\
\text { regulate cell-cell } \\
\text { adhesion }\end{array}$ & 6.65 & 1.77 \\
\hline Caveolin 1 & $\begin{array}{l}\text { Monotopic } \\
\text { integral }\end{array}$ & $\begin{array}{l}\text { Palmitoylation } \\
\text { (3 sites) }\end{array}$ & $\begin{array}{c}\text { Cell } \\
\text { membrane }\end{array}$ & $\begin{array}{l}\text { Caveolae } \\
\text { formation }\end{array}$ & 3.24 & 1.08 \\
\hline $\begin{array}{l}\text { Clathrin heavy } \\
\text { chain }\end{array}$ & Peripheral & & $\begin{array}{l}\text { Cytoplasm / } \\
\text { vesicles / cell } \\
\text { membrane }\end{array}$ & $\begin{array}{c}\text { Major coat } \\
\text { component of } \\
\text { coated pits and } \\
\text { vesicles }\end{array}$ & 10.77 & 1.48 \\
\hline Cortactin & Cytoskeletal & & $\begin{array}{l}\text { Cytoplasm / } \\
\text { cell cortex }\end{array}$ & $\begin{array}{c}\text { Cytoskeletal } \\
\text { element }\end{array}$ & 8.00 & 1.29 \\
\hline Dynamin $1,2,3$ & Peripheral & & $\begin{array}{c}\text { Cytoplasm / } \\
\text { vesicles/ cell } \\
\text { membrane }\end{array}$ & $\begin{array}{l}\text { Vesicular } \\
\text { trafficking, } \\
\text { fission }\end{array}$ & 2.36 & 0.58 \\
\hline ERM & Peripheral & & $\begin{array}{c}\text { Cell } \\
\text { membrane/ } \\
\text { cell cortex }\end{array}$ & $\begin{array}{l}\text { Connection of } \\
\text { cytoskeletal } \\
\text { elements (actin) } \\
\text { to the plasma } \\
\text { membrane }\end{array}$ & 2.14 & 0.53 \\
\hline Flotillin 2 & Peripheral & $\begin{array}{l}\text { N-terminal } \\
\text { myristoylation } \\
\text { and } \\
\text { Palmitoylation } \\
\text { (3 sites) } \\
\text { (Neumann- } \\
\text { Giesen et al., } \\
\text { 2004) }\end{array}$ & $\begin{array}{c}\text { Cell } \\
\text { membrane / } \\
\text { endosomes }\end{array}$ & $\begin{array}{l}\text { Scaffolding } \\
\text { protein within } \\
\text { caveolar } \\
\text { membranes }\end{array}$ & 2.01 & 0.93 \\
\hline
\end{tabular}




\begin{tabular}{|c|c|c|c|c|c|c|}
\hline Munc18-1 & Peripheral & & $\begin{array}{c}\text { Cell } \\
\text { membrane / } \\
\text { cytoplasm }\end{array}$ & $\begin{array}{l}\text { Regulation of } \\
\text { synaptic vesicle } \\
\text { docking and } \\
\text { fusion }\end{array}$ & 2.31 & 0.82 \\
\hline $\begin{array}{l}\text { Nicotinic } \\
\text { acetylcholine } \\
\text { receptors } \\
\text { (probed by } \alpha \text { - } \\
\text { bungarotoxin) }\end{array}$ & $\begin{array}{l}\text { Polytopic } \\
\text { Integral } \\
\text { (four-pass) }\end{array}$ & & $\begin{array}{c}\text { Cell } \\
\text { membrane }\end{array}$ & $\begin{array}{l}\text { Neurotransmitt } \\
\text { er receptor (as a } \\
5 \text {-protein } \\
\text { complex) }\end{array}$ & 4.30 & 0.86 \\
\hline NSF & Peripheral & & Cytoplasm & $\begin{array}{l}\text { SNARE-complex } \\
\text { disassembly, } \\
\text { chaperone }\end{array}$ & 3.79 & 1.38 \\
\hline$\alpha$-SNAP & Peripheral & & Cytoplasm & $\begin{array}{l}\text { SNARE-complex } \\
\text { disassembly }\end{array}$ & 4.50 & 1.22 \\
\hline SNAP23 & $\begin{array}{l}\text { Monotopic } \\
\text { integral }\end{array}$ & $\begin{array}{l}\text { Palmitoylation } \\
\text { (5 sites) }\end{array}$ & $\begin{array}{c}\text { Cell } \\
\text { membrane }\end{array}$ & $\begin{array}{l}\text { SNARE (fusion) } \\
\text { molecule; part } \\
\text { of the } \\
\text { constitutive } \\
\text { secretion } \\
\text { machinery }\end{array}$ & 2.52 & 0.88 \\
\hline SNAP25 & $\begin{array}{l}\text { Monotopic } \\
\text { integral }\end{array}$ & $\begin{array}{l}\text { Palmitoylation } \\
\text { (4 sites) }\end{array}$ & $\begin{array}{c}\text { Cell } \\
\text { membrane }\end{array}$ & $\begin{array}{l}\text { SNARE (fusion) } \\
\text { molecule; part } \\
\text { of the } \\
\text { neurotransmitte } \\
\text { r release } \\
\text { machinery }\end{array}$ & 3.46 & 0.64 \\
\hline Synaptophysin & $\begin{array}{l}\text { Polytopic } \\
\text { Integral } \\
\text { (four-pass) }\end{array}$ & & $\begin{array}{l}\text { Secretory } \\
\text { vesicles }\end{array}$ & $\begin{array}{c}\text { Structural } \\
\text { component of } \\
\text { synaptic vesicles } \\
\text { and dense-core } \\
\text { vesicles }\end{array}$ & 1.41 & 0.63 \\
\hline Syntaxin 1 & $\begin{array}{l}\text { Bitopic } \\
\text { Integral }\end{array}$ & & $\begin{array}{c}\text { Cell } \\
\text { membrane }\end{array}$ & $\begin{array}{l}\text { SNARE (fusion) } \\
\text { molecule; part } \\
\text { of the } \\
\text { neurotransmitte } \\
\text { r release } \\
\text { machinery }\end{array}$ & 1.87 & 0.61 \\
\hline Syntaxin 4 & $\begin{array}{l}\text { Bitopic } \\
\text { Integral }\end{array}$ & & $\begin{array}{l}\text { Plasma } \\
\text { membrane }\end{array}$ & $\begin{array}{l}\text { SNARE (fusion) } \\
\text { molecule; part } \\
\text { of the } \\
\text { constitutive } \\
\text { secretion } \\
\text { machinery }\end{array}$ & 3.10 & 1.11 \\
\hline Syntaxin 13 & $\begin{array}{l}\text { Bitopic } \\
\text { Integral }\end{array}$ & & $\begin{array}{l}\text { Endomembra } \\
\text { ne system }\end{array}$ & $\begin{array}{l}\text { SNARE (fusion) } \\
\text { molecule; part } \\
\text { of the } \\
\text { endosomal } \\
\text { fusion } \\
\text { machinery }\end{array}$ & 4.30 & 1.29 \\
\hline TfnR & $\begin{array}{l}\text { Bitopic } \\
\text { Integral }\end{array}$ & $\begin{array}{l}\text { Palmitoylation } \\
\text { (2 sites) }\end{array}$ & $\begin{array}{c}\text { Cell } \\
\text { membrane / } \\
\text { endosomes }\end{array}$ & $\begin{array}{l}\text { Receptor for } \\
\text { transferrin; } \\
\text { cellular uptake } \\
\text { of iron }\end{array}$ & 6.88 & 0.50 \\
\hline$\alpha$-Tubulin & Cytoskeletal & & Cytoplasm & $\begin{array}{l}\text { Cytoskeletal } \\
\text { element } \\
\text { (microtubules) }\end{array}$ & 8.77 & 1.62 \\
\hline
\end{tabular}


The proteins are described according to the protein database Uniprot, www.uniprot.org, and to references therein. The membrane association is classified as follows:

Monotopic integral proteins: permanently attached to the membrane from only one side.

Bitopic integral proteins: have segments at both sides of the lipid bilayer and permanently attached to it via one transmembrane domain.

Polytopic integral proteins: span the lipid bilayer more than once and permanently attached to it via several transmembrane domains.

Peripheral proteins: temporarily associated with the lipid bilayer through interactions with lipids or membrane proteins.

Cytoskeletal proteins: soluble elements of cortical cytoskeleton.

To determine the location of the proteins within the clouds, line scans centered on the protein clouds were drawn (Figure 3-18B). The line scans confirmed the localization of all the tested proteins within clouds, and also suggested that many proteins enriched in particular areas: some were present mostly at the center of protein clouds, others preferred their edges, while some were randomly distributed within the clouds (Figure 3-18B). In order to have a quantitative comparison of these visible differences in distribution, two parameters were used: fold enrichment of the protein within the cloud and the centerness.

Centerness, which describes the preference of the cloud centers, was determined as follows from the average line scans drawn over immunostained protein clouds. I determined the ratio between the intensity of the immunostained protein and the intensity of the protein cloud at two points in the distribution:

1) The cloud center (Protein intensity center/Cloud intensity $_{\text {center }}$ ).

2) The half-width point of the cloud, representing the edge (Protein intensity edge/Cloud intensity $\left.{ }_{\text {edge}}\right)$.

Cloud center preference was then obtained by dividing the first ratio (measured at the center) by the second one (measured at the edge). This parameter is equal to 1 for proteins that are randomly distributed within clouds, is higher than 1 for proteins that are enriched in the cloud centers, and is lower than 1 for proteins that prefer the edges of clouds. The values obtained are listed in Table 3-1.

For distributions that have one peak (unimodal), categorization was based on their full-width-athalf-maximum. If the line scan showing the distribution of the protein is tighter than that of the protein clouds, the protein has a tendency to be localized at a more central position within the cloud and hence categorized in the "Center preference" group. Proteins having a similar 
distribution to the protein clouds were described as "No preference", since they do not show a tendency for a particular region (Figure 3-18B).

Alternatively, for distributions with 2 peaks (bimodal) categories were separated according to the ratio between the peaks and the central point. If the central point reached above the $60 \%$ of the peak value, the protein was described to show "moderate edge preference". If the central point was even lower than the $60 \%$ cut-off, the proteins had a higher tendency to be localized at the cloud peripheries and are grouped in the "edge preference" category (Figure 3-18B).

A

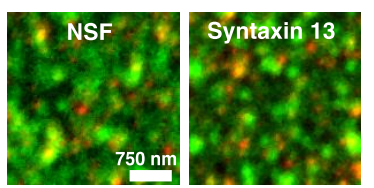

B
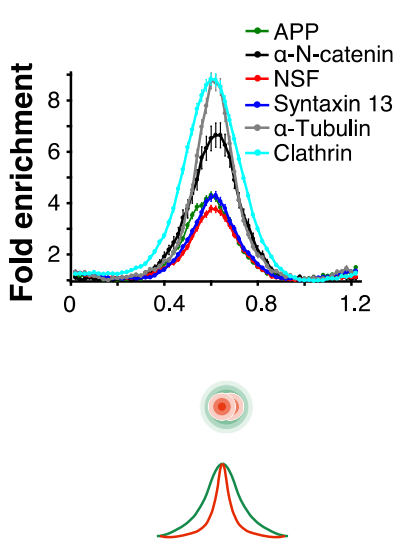
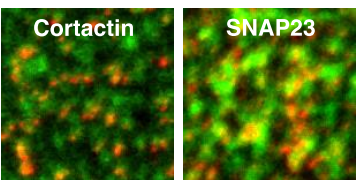

No preference

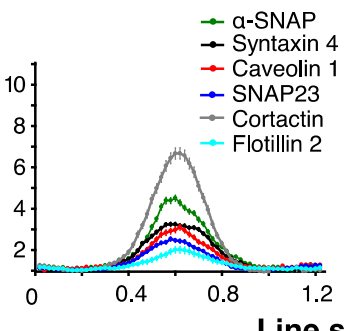

Line scar
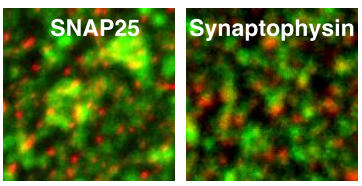

Moderate egde preference
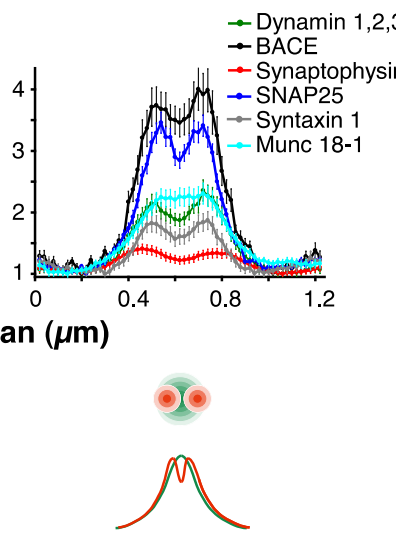
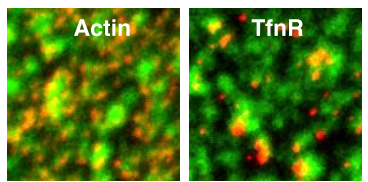

\section{Edge preference}
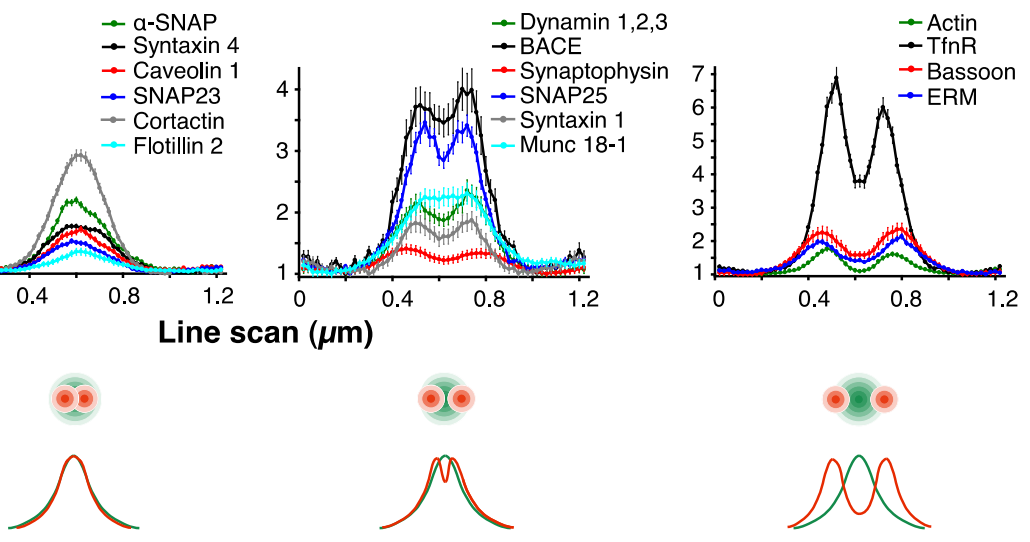

Figure 3-18 Distributions of several specific proteins in the protein clouds.

A. Specific proteins were fluorescently labeled through immunostaining (red) on AHA-containing PC12 plasma membranes (green). The STED images indicate that all these proteins co-localize with the clouds to a good extent but are enriched in different positions relative to cloud centers. B. Analyses of the relative distributions of specific proteins. Unimodal protein distributions were categorized based on their full-widthat-half-maximum: proteins having a tighter distribution than the protein clouds were placed in a "Center preference" category, while those that had similar distributions to the protein clouds were described as "No preference" (no preferred distribution within the clouds). The bimodal protein distributions were categorized according to the ratio between the peaks and the central point. Those where the central point represented more than $60 \%$ of the peak value were described as having a "Moderate edge preference", while those where the central point was below this value were described as having an "Edge preference". The graphs indicate averaged line scans, from 300-900 protein clouds (mean \pm SEM). The two-color drawings at the bottom represent the general distribution profiles for each category (clouds in green, specific proteins in red).

Actin was one of the proteins found farthest from the centers of protein clouds. It appeared to surround them in a ring placed at a distance of $\sim 150 \mathrm{~nm}$ from the centers (Figure 3-18B), where it was joined by ERM (ezrin-radixin-moesin) proteins. ERM are functional partners for actin, serving as links between actin and the plasma membrane (Tsukita and Yonemura, 1997), and thus 
participating in the organization of the so-called "membrane skeleton" (Kusumi and Sako, 1996; Ritchie et al., 2003). This distribution, seemingly forming a barrier (or border) at the edges of protein clouds, is in line with the effects of actin disruption on protein cloud structure (Figure 3-12).

When the borders are lost by actin disruption, clouds can coalesce to form bigger domains (Figure 3-12). To determine whether the overall nature of the clouds remained the same after their coalescence, I have investigated the localization of one of the proteins that preferred the cloud edges, the transferrin receptor ( $T f n R$ ), in membrane sheets treated with low ionic strength buffers which was shown to reduce the actin coverage of the membrane drastically (Figure 3-13) and increase the size of the protein clouds about 3-fold (Figure 3-12). However, TfnR was still found preferentially on the edges of clouds (Figure 3-19). This suggests again that although actin borders the protein clouds, it is not involved in their organization as strongly as cholesterol.

Buffer

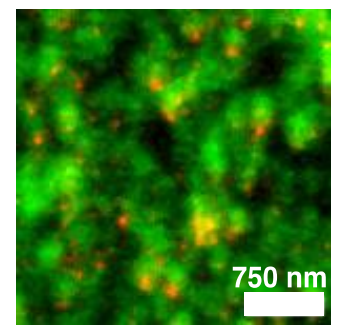

Low ionic strength buffer

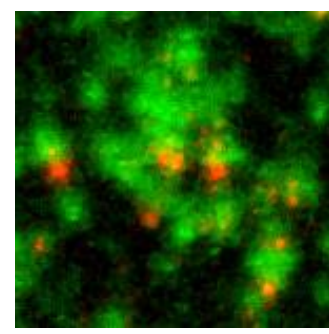

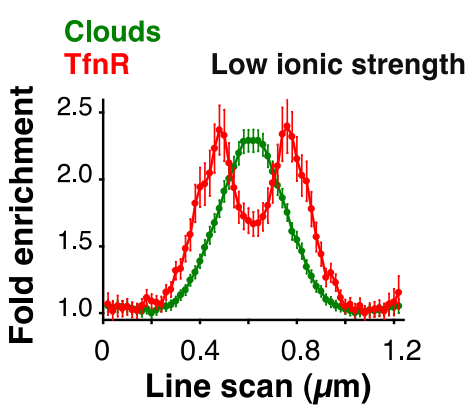

Figure 3-19 Actin disruption does not change the TfnR localization within clouds

STED images and corresponding averaged line scans obtained by immunostaining PC12 membrane sheets for the transferrin receptor (TfnR) after a 20-minute incubation with sonication buffer (control) or with low ionic strength buffer, which causes the formation of bigger clouds. Note that TfnR is still enriched at the edges. $n=493$ protein clouds (mean \pm SEM).

To test whether the distributions I observed were reproducible in other cell types, I performed some of the antibody stainings on COS-7 cells. I observed similar distributions (Figure 3-20), although the enrichment factors were seen to be varying between these different cells. 

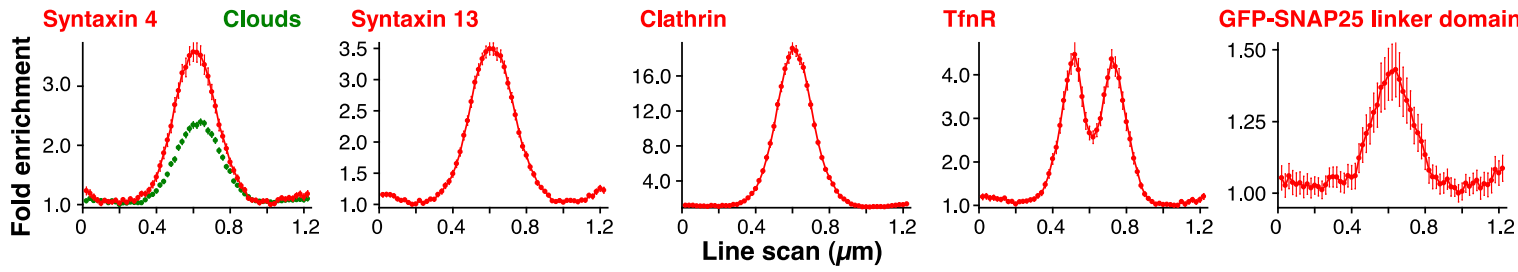

Figure 3-20 Distributions of specific proteins in COS-7 membranes

Specific proteins were fluorescently labeled via immunostaining (red) of PFA-fixed COS-7 plasma membranes (protein clouds - green). The graphs indicate averaged line scans obtained from 385-550 protein clouds (mean \pm SEM).

Since antibodies are known to have the potential of creating small clusters, when possible proteins were also detected on membrane sheets with smaller, monovalent probes. The distribution of syntaxin 1 obtained with antibodies was reproduced using fluorophore-coupled Fab fragments (Figure 3-22). Likewise, an RNA-aptamer reproduced the distribution of TfnR obtained with antibodies in native and fixed sheets (Figure 3-22). In addition, nicotinic acetylcholine receptors were probed with fluorescently labeled $\alpha$-bungarotoxin.
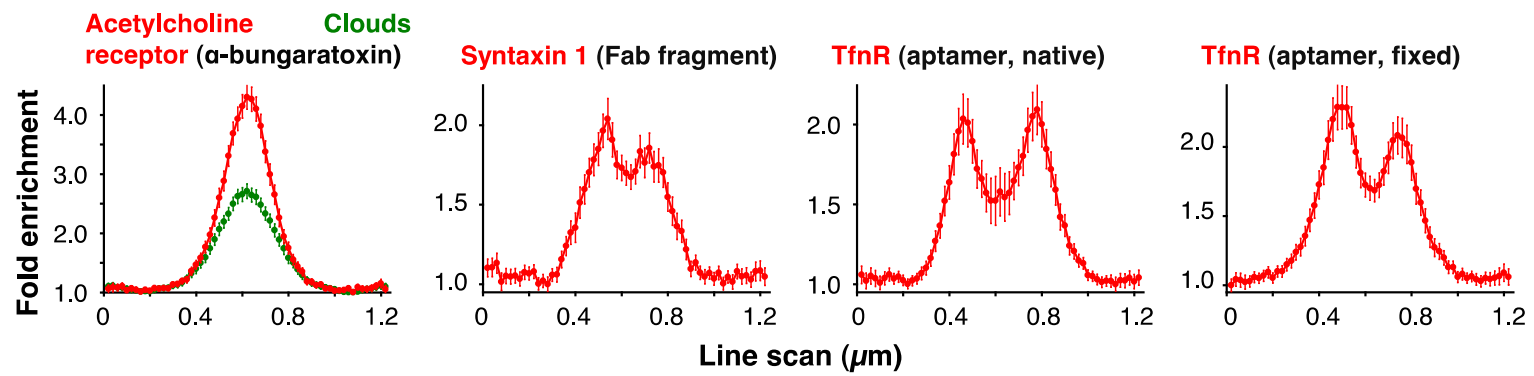

Figure 3-21 Distributions of proteins by monovalent probes

Similar line scans obtained for PC12 membranes by using directly labeled probes such as Atto647Nconjugated $\alpha$-bungarotoxin (to probe nicotinic acetylcholine receptors), anti-syntaxin 1 Fab fragment and TfnR aptamer, instead of full antibodies. TfnR aptamer was applied on living cells prior to sonication and imaged either for fixed membranes or unfixed native membranes. $n=275-600$ protein clouds (mean \pm SEM).

Finally, similar distributions were obtained by immunostaining whole cells (rather than membrane sheets) for proteins that could be targeted by extracellular fluorescent probes, which detected the surface-exposed epitopes of the respective proteins (Figure 3-22). 

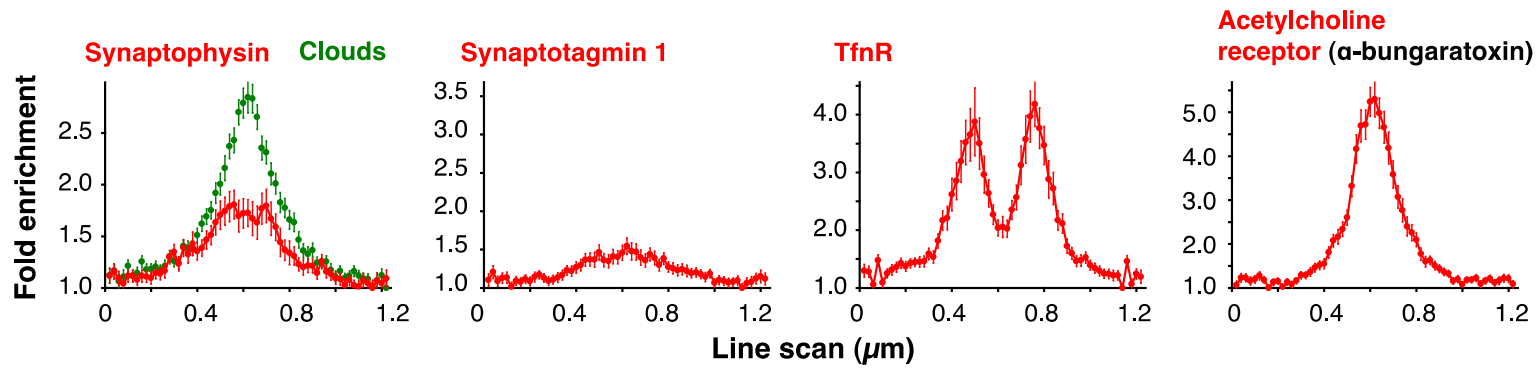

Figure 3-22 Analysis of surface staining of proteins on whole PC12 cells.

Atto647N-conjugated $\alpha$-bungarotoxin was applied onto live PC12 cells; alternatively, antibodies targeting surface epitopes of synaptophysin, synaptogtotagmin, and TfnR were applied to PC12 cells after they were fixed with PFA and glutaraldehyde. Afterwards the cells were labeled by click chemistry and were embedded in plastic resin, as described for Figure 3-4D. 2-color STED imaging was performed on ultrathin sections. The graphs indicate averaged line scans, from 180-240 protein clouds (mean \pm SEM).

\subsubsection{The Effect of Depalmitoylation by DTT}

When a common property for the proteins falling in the same category is sought, one noteworthy observation is the previously reported DRM-association of some of the proteins falling in the center preference and no preference categories, such as caveolin, flotillin (Rajendran et al., 2007; Solomon et al., 2002) and SNAP23 (Salaun et al., 2005a). Following the same pattern, proteins with lower DRM-association such as SNAP25, Syntaxin 1 (Lang et al., 2001; Lang, 2007) and TfnR (Mañes et al., 1999) have tendencies toward the cloud edges (Figure 3-18). The general behaviors of the proteins with lipid anchors were also fitting this observation; palmitoylated proteins which show high DRM-association (Melkonian et al., 1999) had a center preference, whereas the farnesylated proteins known not to be included in DRMs had an edge preference (Figure 3-7A). Although general tendencies indicated a more central localization for raft-like proteins and more peripheral distribution for the non-raft-like proteins, this is hard to establish a wide-ranging rule based on this. Also, general tendencies might not always reflect the distribution of specific proteins. For example, although palmitoylated proteins generally occupied cloud centers (Figure 3-7A), one member of this class, SNAP25, had a moderate preference for cloud edges (Figure 3-18).

An interesting insight was gained by overexpression of a truncated version of SNAP25. This mutant lacks the SNARE domains and is essentially equivalent to the palmitoylated linker domain of SNAP25 fused to GFP (which maintains the overall size of the protein similar to the nontruncated version). Although the endogeneous SNAP25 was detected to show moderate edge preference in PC12 sheets (for comparison the averaged line scan for SNAP25 from Figure 3-18 is replotted in Figure 3-23A), the truncated linker domain overexpressed in COS-7 cells (this cell line 
was preferred due to higher expression efficiency and the lack of endogeneous SNAP25 expression), caused the loss of this tendency (Figure 3-23B). Interestingly, when the membranes were subject to DTT which causes loss of palmitoyl groups (Levental et al., 2010b), the truncated protein was found to be slightly more dispersed suggesting that heavy palmitoylation could be one factor that drives tighter distribution. These results indicate that interactions that are specific to the SNAP25 SNARE domains contribute to its location and palmitoylation might also have a minor role in its distribution, but the localization is governed by neither of them alone.

A noteworthy comparison is between SNAP25 and SNAP23. Although SNAP25 and SNAP23 are structurally very similar, SNAP23 has one more palmitoylation site and was also shown to be more engaged to DRMs (Salaun et al., 2005a; 2005b). In the membrane sheet experiments with PC12 cells, endogeneous SNAP23 was found randomly distributed within the clouds unlike SNAP25, which had a moderate edge preference (for comparison the averaged line scan for SNAP23 from Figure 3-18 replotted in Figure 3-23C). DTT treatment was also applied to SNAP23, but the effect was minor, if any, suggesting that a complex array of interactions govern the distribution of each protein (Figure 3-23D). In conclusion, distribution within the clouds appeared to be strongly influenced by characteristics specific to each protein. 
A

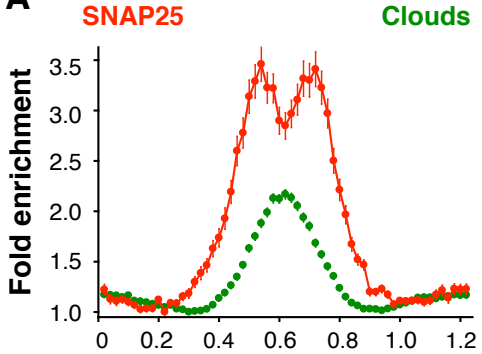

C
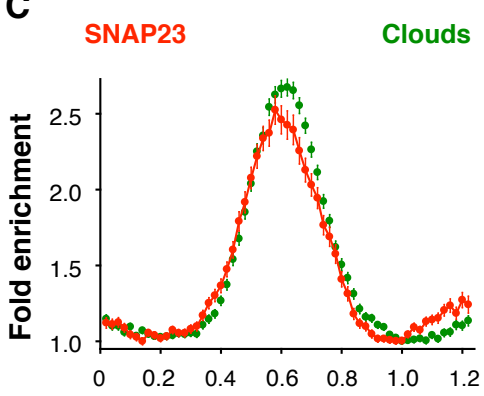

B SNAP25 linker domain (COS-7)

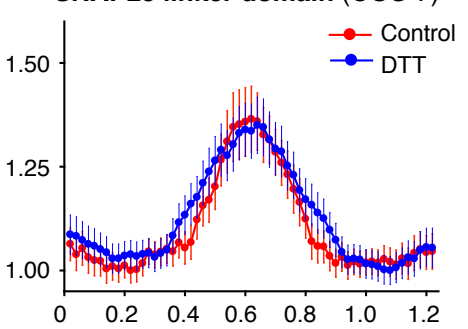

D

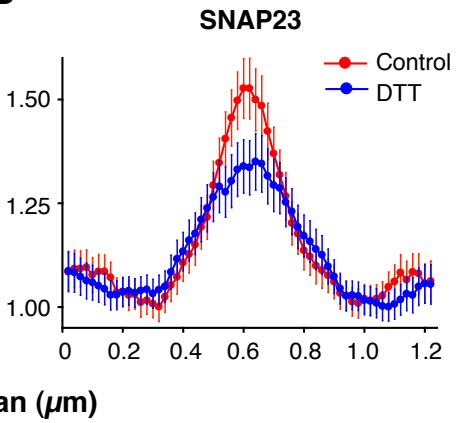

Figure 3-23 Effects of depalmitoylation by DTT.

A. Averaged line scans obtained from PC12 membrane sheets immunostained for SNAP25 (red). The profile of clouds is shown in green. $n=466$ protein clouds (mean \pm SEM). B. The graph shows the overexpression of a truncated version of SNAP25, lacking the SNARE domains and coupled to GFP (thus consisting of GFP coupled to the palmitoylated linker domain of SNAP25) in membrane sheets obtained from COS-7 cells treated with $20 \mathrm{mM}$ DTT (blue) or with ethanol vehicle (control, red) for $1 \mathrm{~h}$ prior to sonication. The protein was detected by immunostaining of click-labeled fixed sheets for GFP. $n=930-1000$ protein clouds pooled from 2 independent experiments (mean \pm SEM). Note the difference from the endogenous SNAP25 distribution (Figure 3-18). C. Same as panel A, but for SNAP23 immunostained sheets. $n=502$ protein clouds (mean \pm SEM). D. Same as panel B but shows the distribution of endogeneous SNAP23 detected by immunostaining of membrane sheets from DTT treated or control PC12 cells. $n=840-1195$ protein clouds pooled from 2 independent experiments (mean \pm SEM).

\subsubsection{Functional Interactions}

To obtain a more quantitative view of the different protein distributions, a scatter plot comparing the preferences of proteins for the cloud centers or their edges ("centerness", see above) versus the level of enrichment in the clouds was generated (Figure 3-24). In this plot, a number of functional groups can be seen. A cohort involved in the fusion of neurotransmitter-containing vesicles to the plasma membrane ( $\mathrm{Ca}^{2+}$-regulated exocytosis) localized to cloud edges (SNAP25, syntaxin 1, bassoon, synaptophysin, their co-factor lipid PIP2; indicated by the blue contour in Figure 3-24. This is in contrast to two proteins involved in the fusion of vesicles from the constitutive secretion pathway to the membrane, SNAP23 and syntaxin 4. These proteins were more randomly distributed, despite their remarkable structural similarity to SNAP25 and syntaxin 1. The endosomal proteins APP and syntaxin 13 both preferred cloud centers (purple contour). Further examples of proteins that seem to localize to similar areas are the functionally related NSF and $\alpha$-SNAP (a chaperone protein and its co-factor, which are involved in the sorting events 
that follow membrane fusion by disassembling SNARE complexes; green contour), and actin and its functional partners from the ERM complex (orange contour).

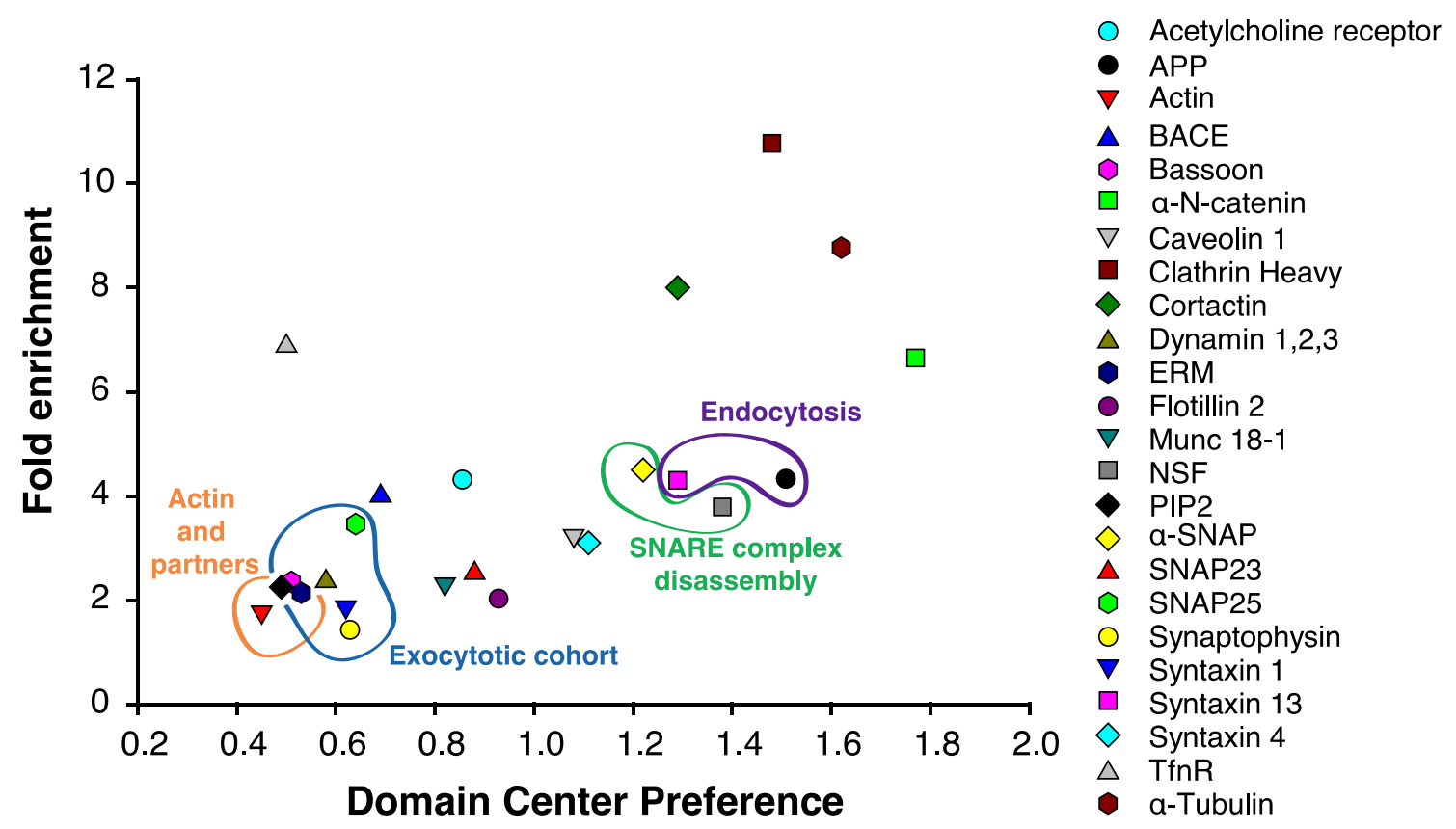

Figure 3-24 Comparison of distributions of specific proteins.

The scatter plot shows the fold enrichment of proteins within clouds, against their domain center preference (the values are listed in Table 3-1). Groups of functionally related proteins are indicated by the colored contours.

These observations suggest that functional interactions might have a strong influence on the distribution of proteins within the clouds and proteins that function together may reach similar regions. This would induce the formation of areas that are highly enriched for those particular protein cohorts, to the point of excluding other components, a situation that may be important for the function of these assemblies.

\subsection{A Technical Note: Development of COIN}

\subsubsection{Secondary Isotope Mass Spectrometry}

As described in Section 3.2, a label-free detection technique, SIMS, was applied to visualize the protein clouds in absence of fluorescent labels. This mass spectrometry-based method is an isotopic imaging technique that is recently gaining popularity especially to study turnover of molecules (Frisz et al., 2013b; Lechene et al., 2006; Senyo et al., 2013; Steinhauser et al., 2012; Zhang et al., 2012). The marker molecules for SIMS contain stable isotopes such as ${ }^{15} \mathrm{~N}$ and ${ }^{13} \mathrm{C}$, which are metabolically incorporated into the cells. The samples are then imaged using as a probe, 
the primary beam of ions such as $\mathrm{Cs}^{+}$(Castaing and Slodzian, 1962). This beam is scanned on the surface of the cellular material to be investigated, causing the sputter of secondary particles, atoms or atomic clusters. A proportion of the secondary particles is ionized and can then be identified by mass-spectrometry (Figure 3-25A). Since the secondary ions are released only from the area hit by the $\mathrm{Cs}^{+}$beam, the lateral resolution of the measurement is only dependent on the size of the beam and reaches $\sim 30 \mathrm{~nm}$ with modern SIMS instruments such as the Cameca NanoSIMS 50L (Senyo et al., 2013). The z-axis resolution is dependent on the mean depth to which the ion beam penetrates into the sample, and is typically $10 \mathrm{~nm}$. Thus, SIMS/MIMS has good spatial resolution and can be used to investigate different cellular structures. Nevertheless, the technique is limited by the fact that with bulk isotopic labeling specific structures cannot be identified at high precision. Some structures, such as nuclei, may be identified by their morphology, but this is not the case for most small organelles. To evade this limitation, one would need to combine SIMS with a method that identifies specific organelles, such as fluorescence microscopy.
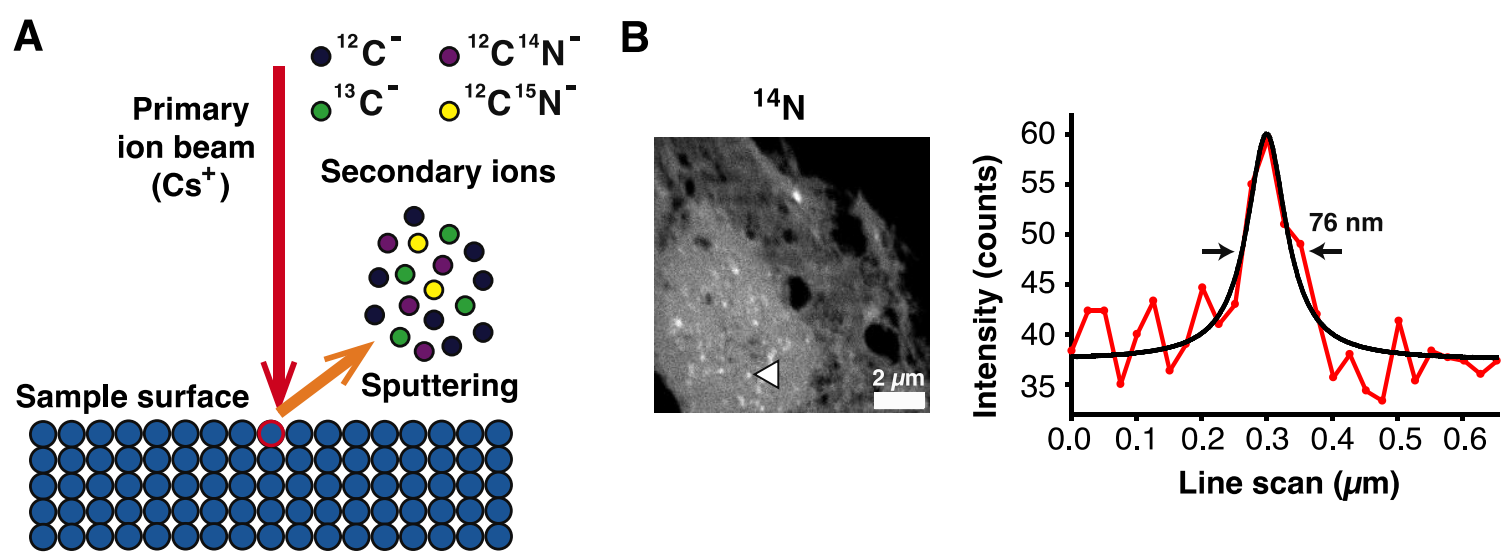

Figure 3-25 SIMS imaging.

A. The primary $\mathrm{Cs}^{+}$beam irradiates the sample, causing sputtering of secondary particles from the sample surface. These partly ionized particles (only a subset of which are depicted in the figure) are then identified by mass spectrometry. B. An example SIMS image of ${ }^{12} \mathrm{C}^{14} \mathrm{~N}^{-}$particles from a neuronal cell body section. The graph shows a line scan (red) along the spot indicated by the white arrowhead. The black curve represents a Lorentzian fit to the line scan with a full width at half maximum (FWHM) of $76 \mathrm{~nm}$. Applying the $84 \%-16 \%$ rule (Lechene et al., 2006), instead of FWHM fitting, gives similar resolution values.

I was also faced with the same problem: to be able to apply SIMS for studies of membrane patterning, I needed to know where the membrane regions are in our samples. I have first tackled this problem by combining SIMS imaging with fluorescence imaging, which resulted in development of a new correlative scheme. To address the disparity between the spatial resolution of SIMS and that of conventional microscopy, super-resolution STED microscopy was used. The resulting approach, termed correlated optical and isotopic nanoscopy (COIN), allows 
the analysis of the turnover of a variety of cellular structures like organelles and membranes, and can be easily applied to different biological systems.

\subsubsection{Correlation of STED Microscopy with Isotopic Imaging}

To correlate STED and SIMS, the biological specimen should be prepared in a form that can be imaged in both setups. The specimen must be stable enough not to suffer any changes between the acquisition of the two images, and it must not be damaged by either of the techniques. As the high vacuum conditions required for SIMS prohibit the use of live cellular specimens, it was necessary to fix and embed the samples in a resin that is suitable for multiple microscopy applications. Although SIMS experiments have previously relied on Epon, a resin originally developed for EM (Lechene et al., 2006), it cannot be used in correlative imaging due to its high autofluorescence. Therefore instead of Epon, LR White resin, which is a polyhydroxy-aromatic acrylic resin with low autofluorescence (Watanabe et al., 2011), was used.

For trials of correlative imaging, cultured hippocampal neurons (Willig et al., 2006) were treated with ${ }^{15} \mathrm{~N}$-labeled leucine for three days. The leucine was incorporated into all the newly produced neuronal proteins, and thus served as a marker for the turnover of neuronal structures. On the fourth day the cells were fixed and immunostained for several organelle markers, including the ER, the Golgi apparatus, mitochondria and synaptic vesicles. The cells were then embedded in LR White resin according to a protocol optimized for the best fluorescence and morphology preservation (presented in detail in Section 2.18.1.2). 200-nm sections were cut using an ultramicrotome and were deposited on silicon wafers (Zhang et al., 2012) as shown in Figure 3-26A, using procedures that have been standardized for electron microscopy (Denker et al., 2009). The use of ultrathin sections, as opposed to whole cells grown on the wafers, has the advantage of increasing the resolution of fluorescence imaging along the vertical axis (otherwise the vertical axis resolution of the typical STED microscope is comparable to that of confocal instruments, about 500-600 nm).

First, the samples were imaged optically using STED microscopy (Figure 3-26B, see Figure 1-5 for details of STED microscopy). As the silicon wafers do not allow the transmission of light, the wafers were placed upside-down on the objective stage, with the sections facing the lens, bathed in immersion oil. This procedure did not harm the samples and allowed efficient excitation and fluorescence depletion. As the exact location of the area imaged by STED needs to be known when the sample is transferred to the SIMS instrument, one needs to create several location 
markers on the specimen. This was achieved by scanning the regions surrounding the imaged area with the multiphoton beam of the Mai Tai laser at maximum energy. The laser induced rapid (3060 seconds) deformation of the LR White resin, selectively in the regions scanned (Figure 3-26C). Markings of different shapes and sizes can be created around the different areas imaged with STED, allowing easy recognition in the SIMS instrument.

A
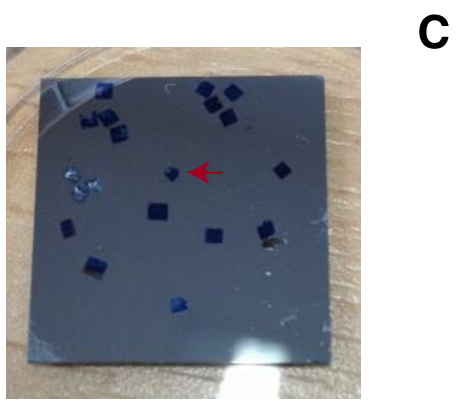

B STED

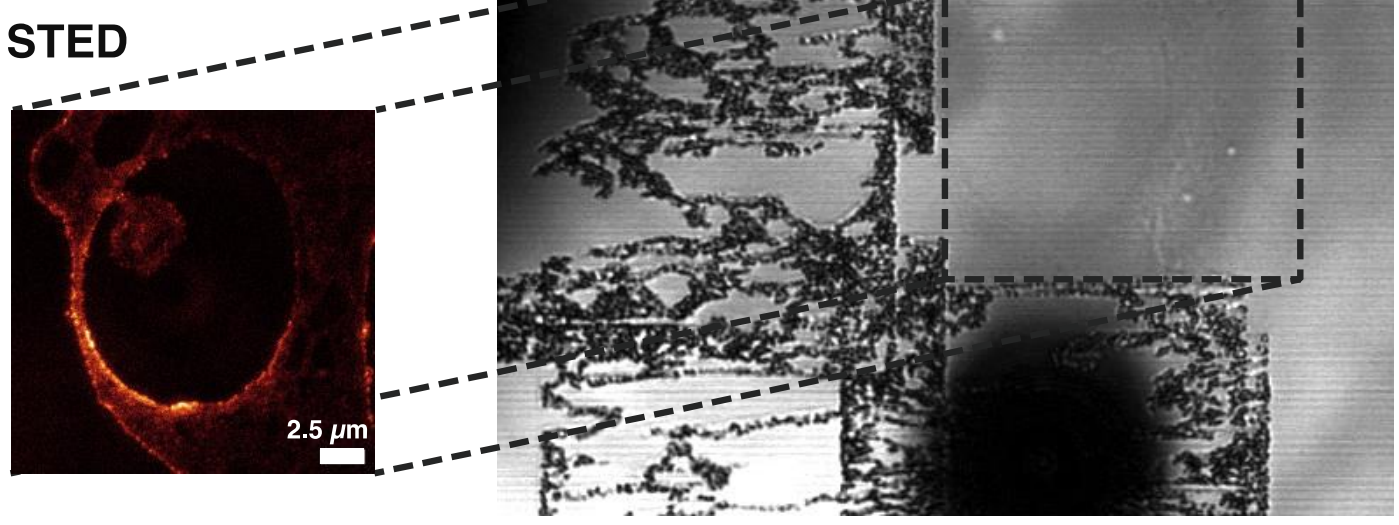

Figure 3-26 Sample preparation for correlative imaging.

A. The cells were embedded in LR White and 200-nm sections are cut with an ultramicrotome. The sections (blue rectangles; one indicated by the red arrow) are placed onto a silicon wafer (gray square). B. The chosen cell area is imaged in the confocal or STED mode (only the STED image is shown here). C. Afterwards the multiphoton laser used for STED depletion (a Spectra-Physics Mai Tai tunable laser) is applied at maximum intensity, at $750 \mathrm{~nm}$, onto the zone surrounding the imaged area, resulting in the heatdependent deformation of the LR White resin. The deformed areas of section can be seen as black markings in a reflection image obtained from the bottom surface of the silicon wafer. The markings can later be recognized using a brightfield camera in the SIMS setup, enabling the identification of the area that needs to be imaged.

The samples were then mounted into a SIMS instrument (NanoSIMS 50L, Cameca). $\mathrm{A} \mathrm{Cs}^{+}$beam raster was scanned over the sample surface (Figure 3-25A), applying a sufficient dose of primary ions to reach the steady-state of secondary ion formation. These ions were then mass separated and detected by using a double focalization mass spectrometer. Nitrogen was detected in the form of $\mathrm{CN}^{-}$ions $\left({ }^{12} \mathrm{C}^{14} \mathrm{~N}^{-}\right.$and ${ }^{12} \mathrm{C}^{15} \mathrm{~N}^{-}$were detected separately, labeled as ${ }^{14} \mathrm{~N}$ and ${ }^{15} \mathrm{~N}$ for simplicity). Also the ratio between ${ }^{15} \mathrm{~N}$ and ${ }^{14} \mathrm{~N}$ was calculated at each location producing the ${ }^{15} \mathrm{~N} /{ }^{14} \mathrm{~N}$ ratio images. As in images obtained in the past in comparable experiments (Senyo et al., 2013; Steinhauser et al., 2012; Zhang et al., 2012), the ratio, and therefore the relative 
incorporation of ${ }^{15} \mathrm{~N}$, is variable in different regions of the cell (close to $\sim 3-8 \%$ of all nitrogen atoms, on average, compared to the terrestrial ratio of $0.366 \%)$.

For a comparison, several organelles of the synapse were targeted (Figure 3-27). The synapse contains neurotransmitter-filled synaptic vesicles, which release their contents by fusing to the plasma membrane at specific sites termed active zones. Synaptic vesicles were identified by immunostaining for synaptophysin 1 , one of their most important components. Active zones were detected by staining one of their major proteins, bassoon (Südhof, 2004). Mitochondria, which deliver the energy necessary for synaptic transmission, were identified by staining for the mitochondrial transport protein TOMM20. Figure 3-27A shows a section through a neuronal branch (axon) in which a synapse, characterized by the co-localization of all three types of organelles (vesicles, active zone, mitochondria), is indicated by white arrowheads. The rest of the cytosol is populated by numerous transport vesicles containing synaptophysin 1 or bassoon, and by several other mitochondria. The turnover of the synapse appears to have been stronger than of the neighboring axon, since the synapse contains higher levels of ${ }^{15} \mathrm{~N}$-leucine than the surrounding axonal space (Figure $3-27 \mathrm{~B}-\mathrm{C}$ ). The regions of high ${ }^{15} \mathrm{~N} /{ }^{14} \mathrm{~N}$ ratio correlate well with the vesicles and with the bassoon signal (Pearson's correlation coefficients of 0.74 and 0.81 , respectively), but correlate only poorly with the mitochondria (Pearson's correlation coefficient of 0.46; Figure 3-27D). This suggests that newly produced vesicles and/or active zone components (such as bassoon) have been transported to this synapse.

Since only a limited number of channels are available for STED imaging, STED imaging was performed only for bassoon, and confocal imaging was used for TOMM20 and synaptophysin 1. Confocal imaging allowed the identification of organelles in the general synaptic area, and even allowed separating the mitochondria from the synaptic vesicle cluster (Südhof, 2004). This is possible since both mitochondria and the vesicle cluster are larger than the diffraction limit. However, the advantage of STED imaging is evident when groups of bassoon molecules, which are much smaller than the diffraction limit, are investigated (Figure 3-27E). The synaptic area contained several groups of bassoon molecules, four of which presented much higher incorporation of ${ }^{15} \mathrm{~N}$-leucine than the others. This level of detail is invisible with conventional microscopy (Figure 3-27F). 


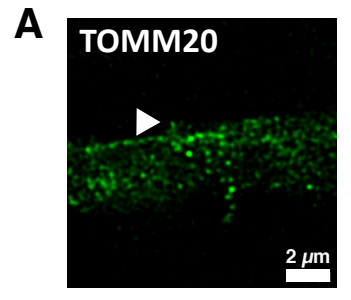

B

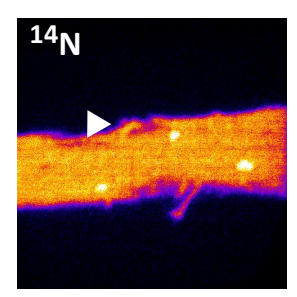

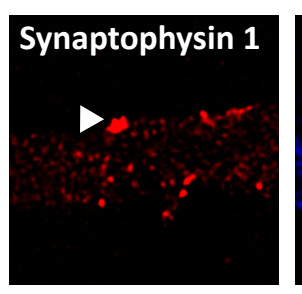
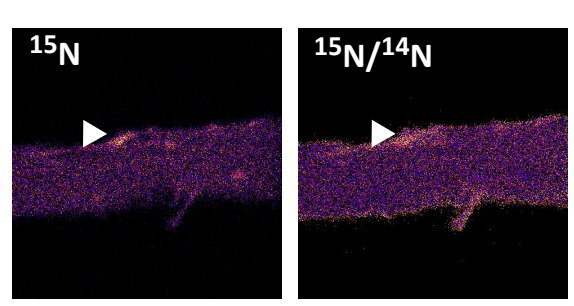
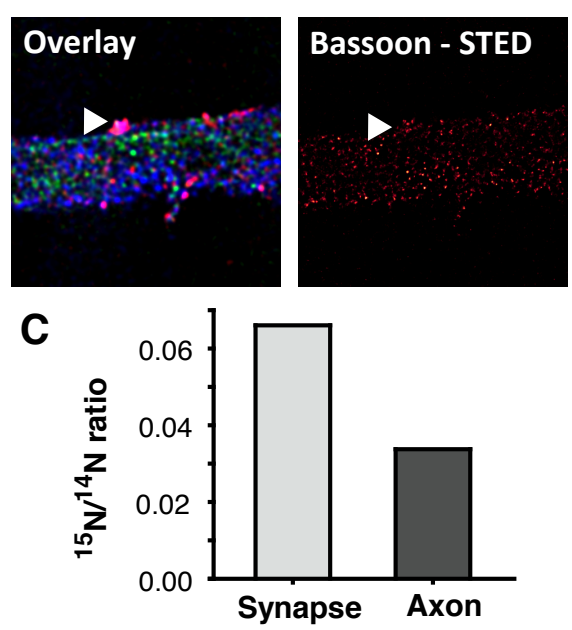

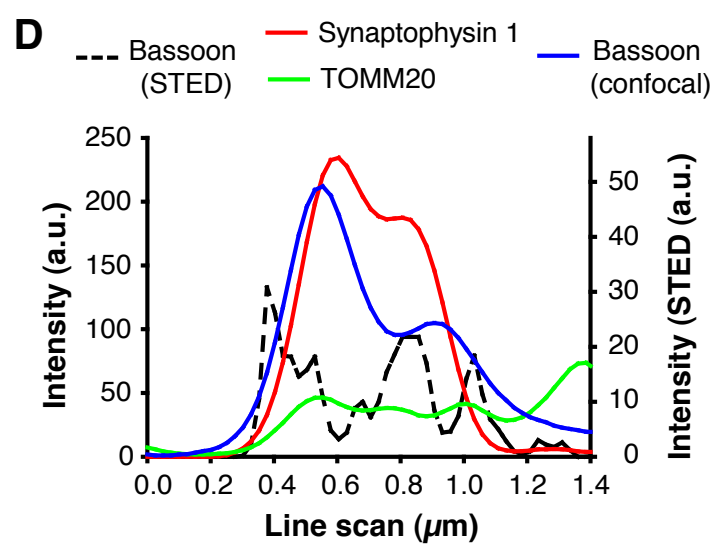

$\mathbf{E}$

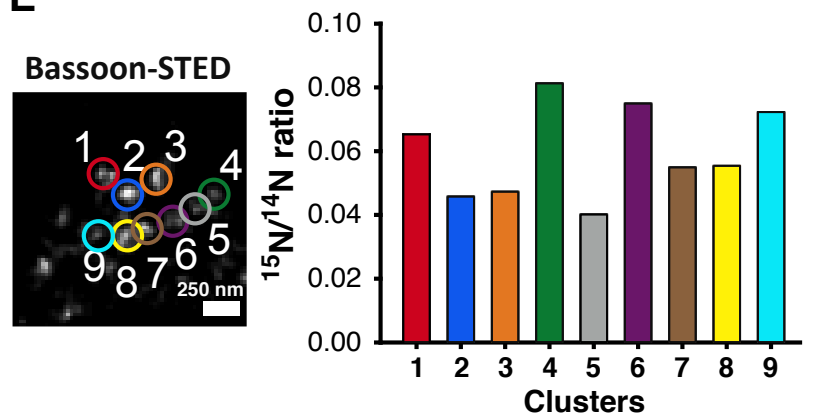

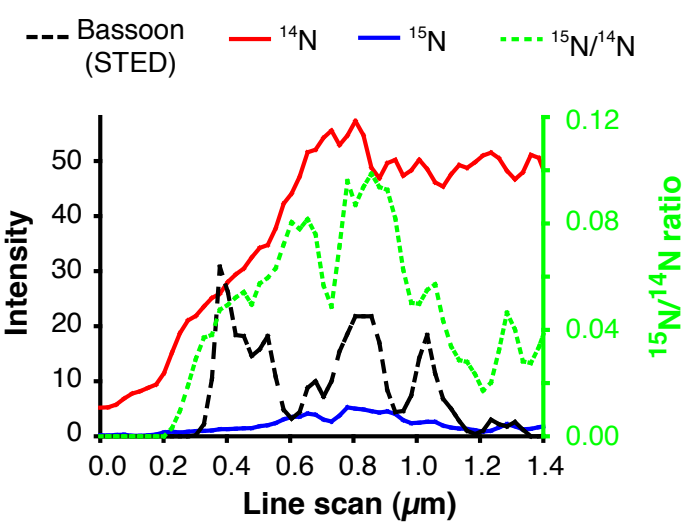

$\mathbf{F}$

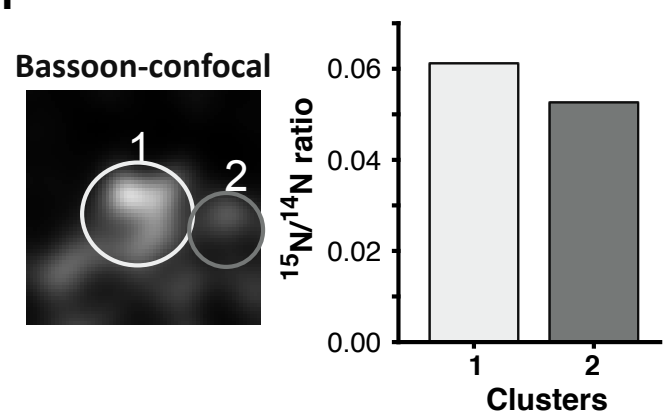

Figure 3-27 Correlative STED and SIMS imaging in neuronal axons.

A. Light microscopy images of a neuronal axon section. The neuron was immunostained for the mitochondrial marker TOMM20 (green, confocal), for the synaptic vesicle marker synaptophysin 1 (red, confocal), and for the active zone marker bassoon (blue, confocal). Right panel displays the STED image for the bassoon labeling. All images were processed by deconvolution. Arrowheads point to a synapse, where all three labels co-localize. B. SIMS images of the same axon for ${ }^{14} \mathrm{~N},{ }^{15} \mathrm{~N}$ and ${ }^{15} \mathrm{~N}{ }^{14} \mathrm{~N}$ ratio, respectively. The color map progresses from purple (low intensity) to white (high intensity). Note the high ${ }^{15} \mathrm{~N} /{ }^{14} \mathrm{~N}$ ratio in the synapse region identified by immunostainings (arrowhead). C. The bars indicate the ${ }^{15} \mathrm{~N} /{ }^{14} \mathrm{~N}$ ratios in the synapse region marked above and in the rest of the axon, respectively. D. Line scans were drawn through the synapse region. The two graphs show the different measurements presented in panels $\mathbf{A}$ and $\mathbf{B}$. The fluorescence intensities are shown in arbitrary units (a.u.), whereas the SIMS data is shown in counts. The SIMS data is smoothed using a moving average spanning 3 values. E. A high-zoom view of the synapse region in the STED channel. Different clusters of bassoon molecules identified within the synapse are numbered 1 to 9 . Their respective ${ }^{15} \mathrm{~N} /{ }^{14} \mathrm{~N}$ ratio values are plotted in the graph. Note that four of the nine clusters have substantially higher ratios. F. The clusters in E visualized in confocal mode. Only two clusters can be identified, with similar ${ }^{15} \mathrm{~N} /{ }^{14} \mathrm{~N}$ ratios. SIMS imaging was performed in collaboration with Angela Vogts (Leibniz-Institute for Baltic Sea Research). 
Next, organelles in the neuronal cell body were investigated (Figure 3-28A). In this case immunostainings were performed for trans-Golgi marker TGN38 and for synaptophysin 1, a marker of synaptic vesicle precursors (both confocal imaging), as well as for the ER marker calnexin (STED imaging). The nucleus, which can be easily identified by its morphology, had the lowest incorporation of ${ }^{15} \mathrm{~N}$-leucine of all identifiable organelles, suggesting that it has the slowest protein turnover (Figure 3-28B-C). In contrast, the trans-Golgi network (TGN38 bar) contained high levels of newly-produced proteins, as did the synaptic vesicle precursors (synaptophysin 1 bar); note that synaptic vesicle precursors overlapped partially with both the Golgi and the ER. Interestingly, the ER (calnexin bar) contained a much lower proportion of new proteins than the trans-Golgi, suggesting that newly-produced proteins leave the ER rapidly, but linger substantially longer in the Golgi apparatus. This is also visible in the line scans in Figure 3-28D, where the Golgi staining (TGN38) correlates best with the ${ }^{15} \mathrm{~N} /{ }^{14} \mathrm{~N}$ ratio (Pearson's correlation coefficient of 0.78 ).

While these measurements seem appropriate for large organelles such as the nucleus and the Golgi apparatus, confocal imaging is no longer sufficient for the diffuse, high-density calnexin staining. For example, the ${ }^{15} \mathrm{~N} /{ }^{14} \mathrm{~N}$ ratio seems to correlate to some extent with the ER staining in the confocal image (calnexin, Figure 3-28E), but the use of STED reveals that the two signals are independent of each other (with even a mild negative correlation detected, Figure 3-28E). This suggests, again, that correlation with super-resolution microscopy is necessary for the investigation of small or densely packed organelles. 
A

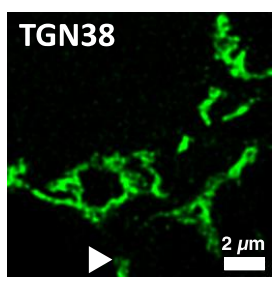

B

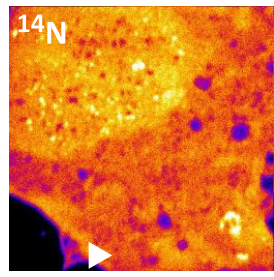

D

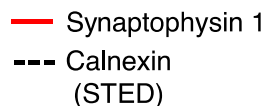
- TGN38
- Calnexin (confocal)

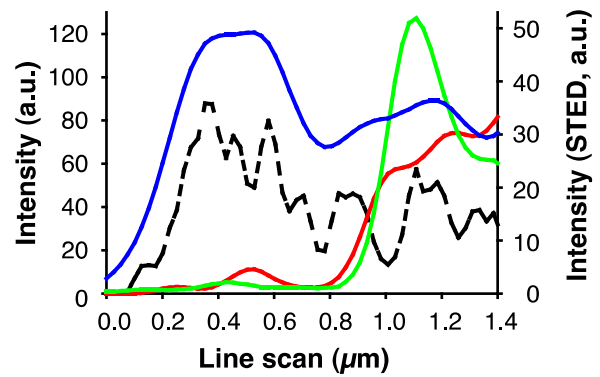

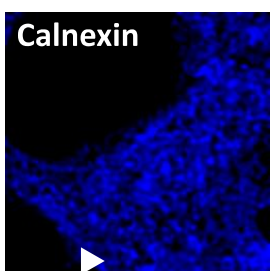
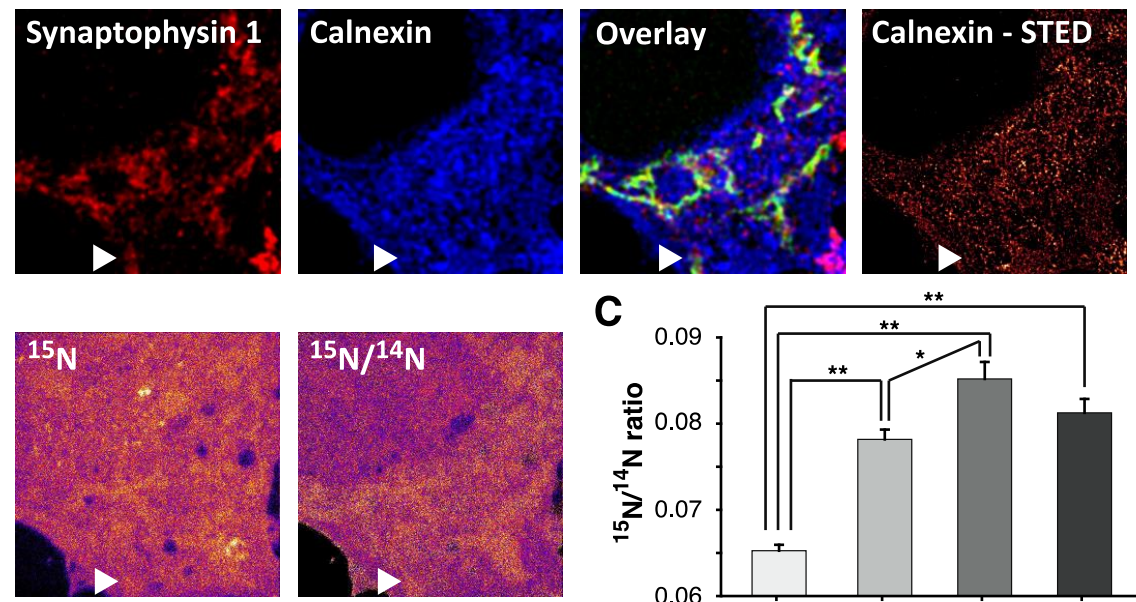

C

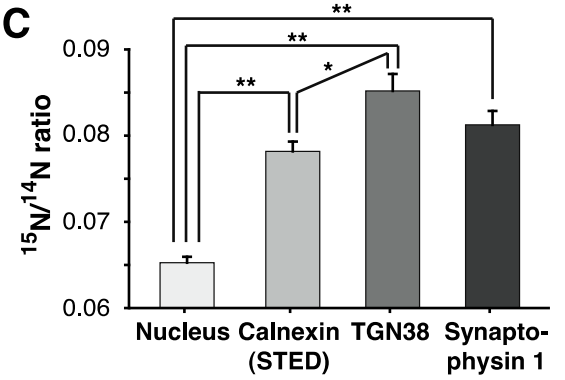

$$
\begin{aligned}
& -{ }^{14} \mathrm{~N} \\
& --- \text { Calnexin } \\
& \text { (STED) }
\end{aligned}
$$

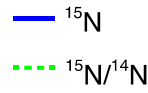

E

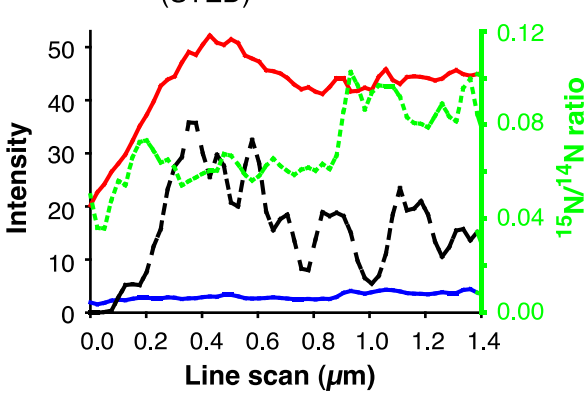

Figure 3-28 Correlative STED and SIMS imaging in neuronal cell bodies.

A. Light microscopy images of a neuronal cell body section. The neuron was immunostained for the Golgi marker TGN38 (green, confocal), for the synaptic vesicle marker synaptophysin 1 (red, confocal; synaptophysin 1 identifies vesicle precursors in the cell body), and for the ER marker calnexin (blue, confocal). Right panel shows the STED image for the calnexin labeling. All images were processed by LD. B. SIMS images for ${ }^{14} \mathrm{~N},{ }^{15} \mathrm{~N}$ and ${ }^{15} \mathrm{~N} /{ }^{14} \mathrm{~N}$ ratio, respectively. Note the low ${ }^{15} \mathrm{~N} /{ }^{14} \mathrm{~N}$ ratio in the nucleus (which is identified by its characteristic morphology, upper left area) and the presence of several cytosolic regions with higher ratios. C. The graph shows the ${ }^{15} \mathrm{~N} /{ }^{14} \mathrm{~N}$ ratio for the different organelles. The values for the nucleus are significantly lower than those for all the other organelles (**, $\mathrm{P}<0.01, \mathrm{t}$-test). Additionally, the ratio is significantly higher in the Golgi apparatus (TGN38) than in the ER (calnexin) ( $*, P<0.05, t$-test). The graphs represent mean + s.e.m, from 26, 57, 9, and 41 regions for the four bars, respectively. D. Line scans were drawn through the region indicated by the arrowheads in A and B. E. Pearson's correlation coefficients were calculated from the line scans in $\mathbf{D}$, to quantify the correlation between calnexin and the ${ }^{15} \mathrm{~N} /{ }^{14} \mathrm{~N}$ ratio, using the STED or confocal images of calnexin. The values were normalized to the maximum expected correlation. SIMS imaging was performed in collaboration with Angela Vogts (Leibniz-Institute for Baltic Sea Research).

Overall, these experiments indicate that correlated optical and isotopic nanoscopy (COIN) is a feasible approach. Each of the two combined technologies provides a piece of information that is unavailable for the other: SIMS yields the material turnover (as indicated by the ${ }^{15} \mathrm{~N} /{ }^{14} \mathrm{~N}$ ratio), while STED microscopy reveals the identities and the spatial distribution of organelles or organelle components. Thus COIN allows a highly precise description of label incorporation at specific sites, enabling turnover experiments for specific structures. These results also validate reliable use of 
these techniques for membrane patterning. Proteins can be detected in a label-free manner by SIMS at the membrane areas identified by fluorescent imaging of an independent membrane marker, in correlative fashion.

Apart from membrane patterning studies, COIN has a large field of potential applications, as it is now feasible to investigate the metabolism and organization of any cellular organelle. Finally, a further potential use of this procedure is the generation of triple-correlation images, since the samples prepared for COIN can also be investigated with scanning electron microscopy (Watanabe et al., 2011) thus allowing a combination of super-resolution fluorescence microscopy, electron microscopy and SIMS on the same sample. 


\section{DISCUSSION}

\subsection{The General Model: Protein Clouds as a Membrane Organizing Principle}

In this study, I have investigated the nanoscale organization of plasma membrane proteins using metabolic labeling and super-resolution fluorescence microscopy. By using plasma membrane sheets, I have observed that the membrane proteins are organized into domains with highprotein abundance, which I have termed protein clouds (Figure 3-3). The cloud pattern was apparent both in PC12 and COS-7 membrane sheets under different fixation protocols and in ultrathin sections from whole cells (Figure 3-4). Label-free visualization of proteins by SIMS based on N14 signal also showed cloud-like heterogeneities, albeit at a lower resolution (Figure 3-5).

The protein clouds were resilient to a variety of treatments including changes in ionic composition and strength, decrease in protein density, disruption of cytoskeletal elements, and hydrolysis of phospholipids and sphingomyelin (Figure 3-11). Manipulations that resulted in actin disruption did not abolish the clouds, but led to formation of larger domains, suggesting that actin is important in preventing coalescence of the clouds. In line with this size-limiting role, actin was also found to surround the clouds. The most striking alteration was seen in conditions that reduce the membrane cholesterol. Depletion of cholesterol almost completely eliminated the protein clouds, in reversible fashion (Figure 3-14). This effect was also reproducible for organellar membranes (Figure 3-15). Early endosomes, which have similar levels of cholesterol, also contained protein domains that dispersed upon cholesterol depletion. In contrast, mitochondria, which have very low amount of cholesterol, seemed to have a rather homogeneous outline. But after introduction of cholesterol, domains were formed. These results suggest that cholesterol is the major factor for the cloud patterning and its action is rather unspecific and indirect. At this point, it is not clear whether cholesterol is concentrated in the clouds; however, the cholesterol analogue I tested (Chol-PEG-KK114) did not show a specific enrichment (Figure 3-8). STED-FCS experiments in living cells showed that inside the protein domains both the cholesterol analogue and proteins were slowed down, suggesting that the molecular crowding in the clouds affects the free diffusion (Figure 3-9).

Generally speaking, the clouds had a high enrichment of proteins containing $~ 81 \%$ of the labeled membrane proteins with a surface occupancy of 50-60\% (Section 3.2 and Figure 3-3). Proteins of 
different ages were found to occupy the same clouds, suggesting stability over time (Figure 3-6). Subsets of proteins with different modifications, as well as individual protein species, were found to be all enriched within the clouds but at varying levels and preferring different locations, such as the edges or centers of the clouds (Figure 3-7 and Figure 3-18). Interestingly, some functional partners were observed to show similar profiles (Figure 3-24).

Based on the distribution measurements presented in Figure 3-18, a circular model was generated (Figure 4-1). This model provides a graphic view of the clouds: the general protein assembly is bordered by actin, and excludes cholesterol to some extent (according to the results with the cholesterol analogue). Different examples of specific proteins are shown, occupying particular zones within the circular cloud, at different distances from the center.

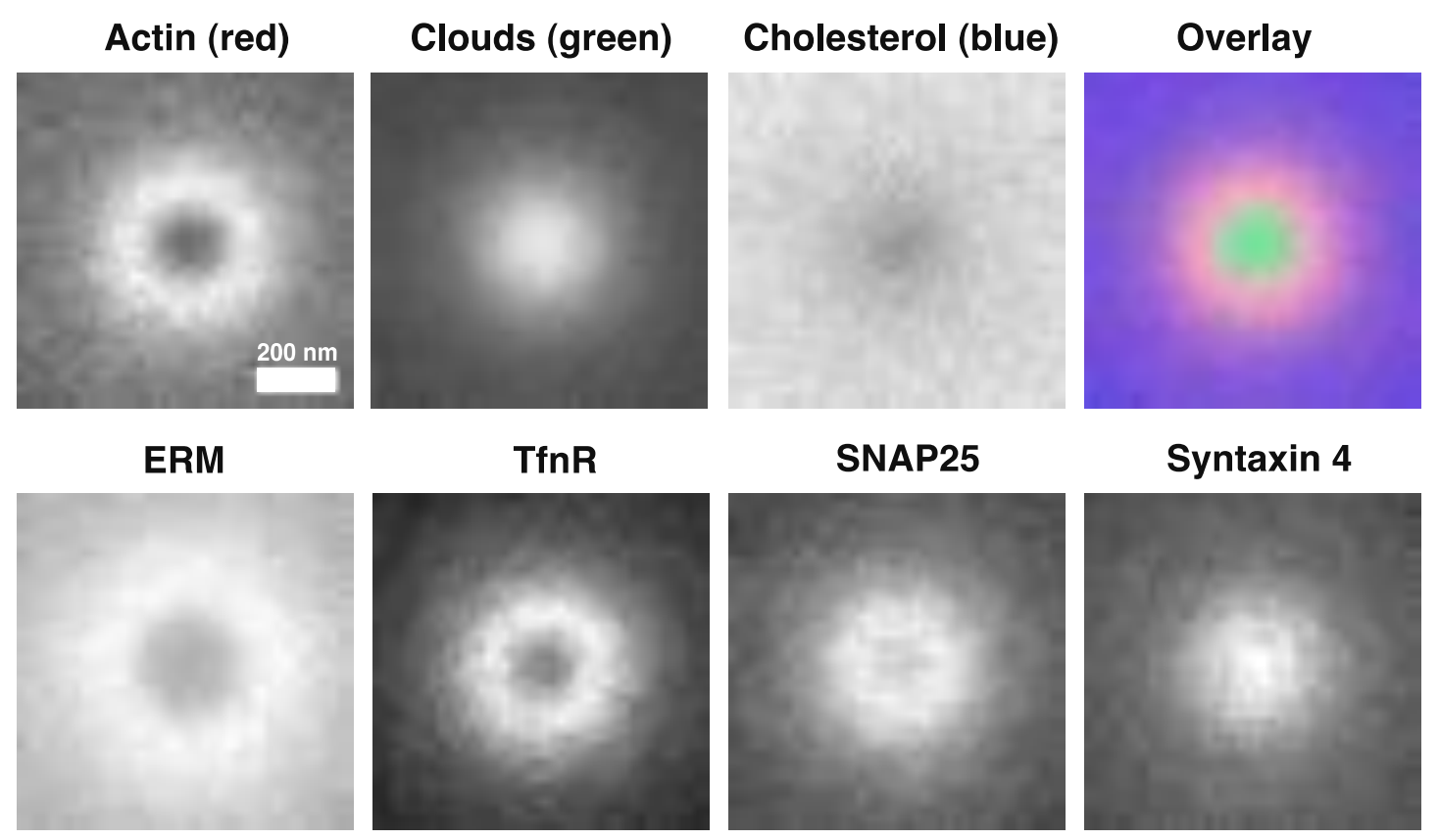

Figure 4-1 Circular model of the protein clouds.

Model protein clouds, organized in circular fashion. The images depict the distributions of clouds (green in the overlay), actin (red) and cholesterol (blue). The clouds are drawn to scale, using information from the line scans in Figure 3-8 and Figure 3-18. Several additional examples of protein distributions are shown in the lower row of panels. The contrast of the images is obtained from the line scans: proteins with a higher enrichment within clouds will appear as brighter spots over a darker background. Elements that are more homogeneously distributed (cholesterol) show less variation in these images.

In essence, our data suggest that the plasma membrane assumes a patterned organization, which is realized via enrichment of membrane proteins in heterogeneous domains, "the protein clouds". The clouds are maintained by the influence of both cholesterol and actin. The latter appears to mainly keep the clouds in place, while at the same time it restricts their size by prohibiting their 
coalescence, in line with the picket fence model of actin-membrane interactions (Ritchie et al., 2003). Cholesterol, on the other hand, acts as a general organizer and seems to be responsible for protein accumulation into clouds. The clouds can collectively create a protein scaffold that allows facilitation of specific and functional interactions. In other words, clouds can act as a basic, lowhierarchy principle of membrane patterning, underlying the distributions of specific proteins.

\subsubsection{The Determinants of the Clouds}

A fundamental question in the membrane organization field is about the main driving force behind general patterning. Is it lipid-driven as suggested in the concept of lipid rafts or rather protein-driven as expected in cytoskeleton-based models such as picket-fence, actin-flows or protein islands (Gowrishankar et al., 2012; Kusumi et al., 2011; Lillemeier et al., 2006; Lingwood and Simons, 2010)? The results I have presented favor a model of cholesterol-driven first level organization of proteins, giving rise to the protein clouds. How cholesterol achieves this is not completely clear.

Two general scenarios are possible: first, specific interactions of cholesterol with certain lipids and proteins might drive segregation of the interaction partners into the observed protein clouds. Second, high levels of cholesterol may change the membrane properties favoring clustering of proteins together and their separation from the rest of the membrane. The first scenario, partitioning of proteins into membrane domains enriched in cholesterol and sphingolipids like in the case of membrane rafts (Lingwood and Simons, 2010; Pike, 2006), is not by itself sufficient to explain the formation of the protein clouds, due to a number of reasons: 1) The protein clouds do not appear to be enriched in the cholesterol analogue we tested (which is even slightly excluded, Figure 3-8). This is in agreement with recent isotopic imaging results that indicated homogenous distribution of cholesterol in the plasma membrane (Frisz et al., 2013a). 2) Depletion of sphingomyelin does not result in cloud dispersion. 3) Several proteins known to be 'non-raft' proteins (TfnR, syntaxin 1, SNAP25) are enriched in the clouds. 4) Raft domains are typically thought to affect only a specific subset of proteins and not the majority of the membrane proteins. Therefore, although membrane rafts might be involved in higher levels of protein organization, the clouds per se are not identical to rafts.

The second scenario, in which membrane proteins are indirectly driven into clusters by the effects of cholesterol on the lipid bilayer, could at least account for the very general impact of cholesterol on protein distribution we observed here. It is also the only explanation that covers the effect of 
cholesterol addition to membranes that lack native cholesterol (mitochondria), which induces protein clustering similar to the plasma membrane (Figure 3-15). This is in line with studies on membrane peptides in artificial systems, which showed that physiological levels of cholesterol significantly increase membrane thickness and at the same time stiffen lipid acyl chains, thus constraining conformational freedom at the segregation of proteins into protein-rich domains where protein-protein contacts are increased (de Almeida et al., 2004; de Meyer et al., 2010; Kaiser et al., 2011; Murray and Tamm, 2011). Also, the lipid shell concept suggests a similar effect from the perspective of proteins inducing local lipid disturbances (Kusumi et al., 2011; Marsh and Páli, 2004; Poveda et al., 2008). As a result, it seems that the driving force is a combined effect of presence of proteins and cholesterol in the bilayer together at high densities.

Higher levels of organization is expected to be driven by more specific protein-protein and protein-lipid interactions, as indicated by the finer organization within the clouds and differential enrichment of proteins, as well as actin-dependent effects. Based on previous studies, electrostatic interactions (Zilly et al., 2011) and potential lipid domains (Frisz et al., 2013b; Fujita et al., 2007; 2009; Simons and Sampaio, 2011) are also predicted to contribute to the organization.

\subsection{Implications of the Protein Clouds}

\subsubsection{Implications Regarding the Membrane Organization}

The protein clouds appear to be the molecular basis for the lateral organization of specific proteins. As noted in Section 1.6, for many proteins, clusters are arranged in a defined pattern, rather than being scattered randomly (Figure 1-6). The data that I present provides a mechanism for this observation: protein clouds segregate the membrane in irregular protein-rich patches, bordered by protein-poor areas. Specific membrane proteins will organize according to this model, resulting in a non-random, ordered pattern. Moreover, as indicated in Figure 3-18, specific proteins will be confined to particular locations within the protein clouds.

A main advantage of enriching proteins in the clouds can be providing spatiotemporal regulation to signaling events, dynamic functions such as exo-/endocytosis and enzymatic reactions. Interestingly, a recent simulation about the effect of confined membrane domains on enzymatic reaction mechanisms put forward that confinement of proteins causes a change in the temporal pattern of reactions. Instead of low yield constant reactions that would happen in a uniform 
membrane, reaction bursts can be created through confinement (Kalay et al., 2012). When it is considered that most of the reactions taking place in the membrane are reversible (like phosphorylation/dephosphorylation, unbiquitination/deubiquitination, activation/deactivation), the importance of promoting bursts rather than a constant level of low-yield reactions becomes easier to appreciate. So cloud enrichment of proteins can help creating bursts that allow the cell to rapidly initiate robust signaling cascades before the triggering reactions are reversed by competing enzymes.

In addition to this general enrichment, further organization of specific proteins within the clouds has other implications. The fact that proteins from the same structural class (for example the SNARE fusion proteins SNAP23 and SNAP25, or syntaxin 1, syntaxin 4 and syntaxin 13) can have different distributions suggests that interactions particular to each protein, rather than broad structural effects, determine the localization of a protein within the cloud. The result that several proteins with functional relevance prefer the same areas supports this idea (Figure 3-24). For example, bassoon, Munc18, syntaxin 1 and SNAP25 co-localize at the edges of protein clouds. Their involvement in fusion with synaptic-like microvesicles or dense-core vesicles loaded with neurotransmitter molecules (Bonanomi et al., 2006) requires that they occupy areas where fusion can take place rapidly and efficiently to allow the speedy incorporation of the incoming vesicle into the plasma membrane. It is probably difficult for this event to take place in the protein-dense cloud areas. Thus the co-enrichment of these exocytotic proteins close to the cloud periphery can fit with the possibility of utilizing these areas for vesicle fusion. In contrast, SNAP23 and syntaxin 4 are involved in constitutive secretion, which takes much longer than neurotransmitter exocytosis. Constitutive secretion may not require the pre-assembly of the fusion machinery on cloud edges. SNAP23 and syntaxin 4 could therefore be randomly distributed, only to be recruited to the secretory vesicle after its docking to the membrane. Localization can also be easily understood in functional terms for synaptophysin, a component of synaptic vesicles and microvesicles (Bonanomi et al., 2006; Takamori et al., 2006), that is also enriched at the cloud edges. This places it in an ideal position to be retrieved from the plasma membrane (endocytosis), separated from many other membrane proteins. According to this view, it might be contradictory that proteins of the endocytotic machinery, such as clathrin and caveolin appear not to show strong edge preference. However, it is probable that clathrin and caveolin in the membrane are found mostly in coated pits that are uniformly covered with these molecules. These structures likely form specialized protein clouds, with a defined function (endocytosis). 
On a different note, the functional compartmentalization might not be limited to protein-dense clouds, but also extend to protein-poor regions. The protein-poor labyrinth surrounding the protein clouds can potentially function like a corridor where proteins escaping the clouds can diffuse faster and move more effectively. These more "flexible" regions may also be used for processes that involve substantial membrane rearrangements, such as endocytosis and exocytosis (as discussed above), since the membrane here may be more easily modified than the proteindense areas. Supporting this statement, previous studies which reported presence of dark osmium-stained patches by EM and raised domains by AFM in mast cells identified budding sites like coated pits at the periphery of these structures which are thought to be rich in proteins (Frankel et al., 2006; Wilson et al., 2004).

\subsubsection{Experimental Implications}

Since this study mainly deals with very general aspects of membrane organization, some of the results point to broad implications regarding general experimental strategies.

One experimental implication of the cloud organization is that it is not possible to reach a conclusion of functional partnering for proteins solely on the basis of (diffraction-limited) colocalization of two proteins. Being found in the same cloud might not necessarily mean that proteins function together, especially because there seems to be a second level of organization within the clouds. However, when it is considered that most clouds are rather small with a median diameter of $\sim 80 \mathrm{~nm}$, it is possible that this sub-cloud organization is not revealed by conventional light microscopy.

A related case can be made for the overexpression of proteins for localization studies. I have observed that proteins are spilled into the protein-poor regions when they cannot be fit into the clouds due to protein overexpression (Figure 3-10). This result shows that overexpression in general might cause significant alterations of the nanoscale distribution of proteins and can lead to inaccurate conclusions.

Another implication regards the evaluation and interpretation of results related to cholesteroldependent clustering of specific proteins. When a protein is predominantly found in DRMs or when its distribution is sensitive to cholesterol depletion, a common tendency is to expect a direct interaction of the protein with cholesterol or their co-partitioning in $\mathrm{L}_{0}$ lipid rafts. As have also been criticized in the past, drawing a direct link might not always be accurate (Kenworthy, 
2008; Simons and Sampaio, 2011). Indeed, the reversible loss of protein clouds in the plasma membrane and their cholesterol-driven formation even in membranes that normally do not have much cholesterol (as for mitochondria), points to a very general organizing role of the cholesterol. These results indicate that cholesterol can influence the clustering of a broad range of proteins in an unspecific and indirect manner. This broad effect I propose is in accordance with a previous study where diffusion of raft and non-raft markers were systematically measured by FRAP in living cells. Cholesterol depletion with MBCD was found to decrease the diffusion for both raft and nonraft markers (Kenworthy et al., 2004). What is interesting is that, besides physical extraction of cholesterol, its chemical modification via COase also had a similar cloud dispersion effect, although to a lower extent (Figure 3-14). Enzymatic oxidation of cholesterol yields 4-choleren-3one. It seems that this small modification is enough to reduce the cloud forming ability of cholesterol. Therefore, in addition to specific interactions, it would be helpful to consider these more general effects of cholesterol in organizing proteins while evaluating the results of cholesterol depletion experiments.

\subsubsection{General Biological Implications}

\subsubsection{Cholesterol in Health and Disease}

A wide range of health conditions including aging and neurodegenerative diseases are found to be associated with changes in the level or asymmetry of cholesterol in the plasma membrane (Di Paolo and Kim, 2011; Simons et al., 2001). Changes in cholesterol metabolism or transport might have diverse consequences (Orth and Bellosta, 2012). One specific case, in the context of Alzheimer's disease, can be APP processing. Normally $90 \%$ of APP is processed by $\alpha$-secretase which directs the non-plaque forming pathway, whereas $10 \%$ is cleaved by BACE leading to A $\beta$ formation in what is known as the amyloidogenic pathway (Marlow et al., 2003). The differential enrichment profiles I have observed for APP (center preference) and BACE (moderate edge preference) are in line with their low interaction on average (Figure 3-18). This interaction is largely accepted to occur in lipid rafts, since cholesterol depletion was observed to decrease the $A \beta$ production (Canevari and Clark, 2007; Simons et al., 2001), although there are other studies reporting that raft-localization is not obligatory for the APP cleavage (Vetrivel et al., 2009). In any case, there seems to be a direct link between membrane cholesterol levels and $A \beta$ generation ( $\mathrm{Di}$ Paolo and Kim, 2011). In addition to the lipid-raft dependent regulation and many other links between lipids and APP processing (Di Paolo and Kim, 2011), the general arrangement of these 
proteins in the clouds with respect to each other might be important for part of the susceptibility to changes in cholesterol level.

In general, when the very broad and unspecific effect of cholesterol on protein clouds is considered, the consequences of alterations in membrane cholesterol level might not be only limited to specific cholesterol interactions or disruption of lipid rafts (Ehehalt et al., 2003); but at least some effects can also be attributed to this general organizer role of cholesterol. Taking this into account can open way for new ideas for investigation of molecular mechanisms for some of these diseases.

\subsubsection{Membrane Domains in Yeast and Bacteria}

The very basic effect I observe for cholesterol suggests that similar effects can be seen with other sterols in different organisms. For yeast cells, which mainly contain ergosterol, a wide range of proteins were found to be in different domains, which were largely altered in sterol mutants (Spira et al., 2012). However, in yeast membranes there might be various other factors such as the presence of a cell wall, different actin dynamics and slower protein diffusion that makes a direct comparison of the membrane organization difficult (Spira et al., 2012). A previously observed feature of the yeast membrane is the large stable protein complexes called eisosomes (Walther et al., 2006). These intriguing structures seem to be formed by self-assembly of highlyabundant proteic components into protein scaffolds, which can bind specific phospholipids (Karotki et al., 2011). Unlike the more heterogeneous protein domains and patches, these furrowshaped protein complexes seem to be more specific to the yeast membrane and are subject to a quite different organization than the protein clouds.

Prokaryotes present an interesting comparison, since they do not have sterols or sphingolipids in the membrane. The well-known examples of prokaryotic membrane domains are those involved in photosynthesis and bioenergetics like chromatophores, chlorosomes, or the purple membrane of halophilic archea formed by crystalline patches of bacteriorhodopsin (Boekema et al., 2013). In comparison to the clouds these domains are quite different that they create fairly specialized sites, made up of very few protein species. In comparison to the clouds, these domains are without doubt quite different in nature. However, in bacterial membranes indications of lipid microdomains and DRMs were also observed. One example is the cardiolipin-rich domains detected at the poles of the cells and the minicells that bud from the poles (Mileykovskaya and Dowhan, 2009). Furthermore, flotillin 1-like proteins were discovered to be widely expressed and 
distributed in a punctate pattern in sporulating bacteria. Such domains are distinct from cardiolipin-rich domains and can confer detergent-resistance. Squaelene-derived polyisoprenoids were found to be important lipidic factors for these so-called bacterial lipid rafts (López and Kolter, 2010). Interestingly, there are also some exceptional bacterial species such as Borrelia, which contain cholesterol and its derivatives like cholesterol glycolipids (LaRocca et al., 2010). These species were also shown to bear DRMs containing some of the outer membrane proteins, which showed susceptibility to sterol depletion (LaRocca et al., 2013).

In contrast, proteins in the mitochondrial membranes, which are poor in cholesterol, were shown not to be sensitive to cholesterol depletion and are also not found in DRMs (Zheng et al., 2008). Likewise, in our labeling conditions general protein domains in the mitochondrial membrane (at least in the outer membrane, which is more accessible to the click labeling) were not as evident as those in the plasma membrane or early endosomes (Figure 3-15). Therefore, it is possible that functional membrane domains in mitochondria are much smaller than the plasma membrane clouds. They might be more on the order of specialized protein complexes, dependent on specific protein-protein, protein-lipid interactions or electrostatic forces. Interestingly, cardiolipin, was found to be indispensible for long-range row-like assembly of ATP synthase complexes in cristae membranes and functioning of the respiratory chain (Acehan et al., 2011; Dudkina et al., 2010). In conclusion, these results suggest that alternative mechanisms of membrane specialization might be acting in membranes of different composition and finding conserved rules might not be very straightforward.

\subsection{Experimental Rationale and Limitations}

\subsubsection{Metabolic Labeling}

To address the general protein distribution, I have exploited metabolic labeling, which targets all proteins indiscriminately. For that I have relied on incorporation of alkyne or azide containing methionine analogues, AHA or HPG, in the absence of normal methionine. A similar type of metabolic labeling with AHA has been applied before for shorter incubation times to study protein turnover (Dieterich et al., 2007). This previous study established that AHA incorporation is not toxic to cells and the specificity and extent of click labeling with AHA incorporation is comparable to labeling with radioactive amino acids. They could not find any evidence that AHA incorporation alters global protein synthesis or degradation rates. Moreover, AHA-labeled 
histones were localized to proper subcellular locations (Dieterich et al., 2006). In my experimental conditions, I have also confirmed the overall performance of cells through assays of membrane trafficking and differentiation (Figure 3-2). Additionally, AHA-treated cells were able to target their proteins to different membranes (plasma membrane, endosomes and mitochondria) as shown by immunostainings, and were also able to express and sort exogenous proteins in high levels (Figure 3-10). These suggest that incorporation of the methionine analogue does not disturb the sorting and subcellular localization of proteins. Furthermore, I have employed analogues of two different chemistries to account for possible difficulties created in the protein structure due to incorporation, but I have not observed a major difference in the labeling pattern for the two analogues (Figure 3-6).

For metabolic labeling, methionine is a good target; it is an essential amino acid and in human proteome and most proteins contain at least four methionine residues (Dieterich et al., 2006). The fact that methionine constitutes on average only $2.2 \%$ of the amino acids in a protein (rat and human genomes) in comparison to $10 \%$ for an abundant amino acid like leucine (Tekaia and Yeramian, 2006; Tekaia et al., 2002) is also considered an advantage, since over representation of single proteins labeled at many sites become less likely. Also, to ensure that all proteins incorporate at least one AHA, the incorporation was continued for 72 hours, which is roughly equal to 2 times the median proteome half-life determined for human (HELA cells, 35.5 hours) and mouse cell lines (C2C12 cells, 43.18 hours) by stable isotope labeling (Cambridge et al., 2011).

Yet on a quantitative level, I cannot neglect the possibility that not all proteins might be equally represented by AHA incorporation and by click labeling. Nevertheless, our labeling should be representative of the global membrane proteins for a qualitative assessment of distribution. This is demonstrated in different ways. If the proteins collected from AHA-treated cells are subject to PAGE, many bands can be click labeled (Figure 3-1). Also, combination of click labeling with immunostaining against various proteins (Figure 3-18, Figure 3-20) or double labeling of general proteins and modified subsets of proteins (Figure 3-7) gave a good overlap suggesting that many different proteins are found in the AHA-labeled structures.

For the AHA incorporation to be efficient, cells are grown with dialyzed serum. A recent study showed that yeast in stationary growth phase produce stable micrometer-scale lipid and protein domains as a result of a stress-induced pathway (Toulmay and Prinz, 2013). However, I do not expect that the protein clouds are such stress-induced structures. First, no similar stress-induced large-scale segregation phenotype has been described in mammalian cells. Second, even with 
dialyzed medium the medium composition of cells should have plenty of essential nutrients for normal functioning. Third after three days of AHA incubation in dialyzed serum, cells can perform membrane trafficking at normal levels and can undergo differentiation suggesting that they are not experiencing extensive starvation-related stress.

\subsubsection{Click Reactions and Fluorophores}

Copper-catalyzed click labeling I have employed is a highly efficient and specific cycloaddition reaction. As obvious, a reaction that would avoid copper would be more desirable. However, in preliminary experiments I have observed that the copper-free click reaction, which required structurally more complex strained cyclooctyne functionality, was not as efficient nor as specific as the copper(I)-catalyzed reaction. Therefore, although it is less compatible with live labeling, copper-catalyzed click reaction was preferred. To prevent an adverse effect of copper on protein distribution, click reaction was always performed after fixation (except some control conditions like in Figure 3-4).

An advantage of the click labeling is that it works 1:1 and does not create crosslinking of targets. However, one cannot neglect a potential affinity between fluorophores, which can induce clustering. To avoid that fluorophore coupling was performed after fixation. Also, click reactions were performed using azide or alkyne modifications with two chemically different dyes, Atto647N and Chromeo494. The general cloud pattern was not different for the two dyes. Furthermore, SIMS imaging was performed to image the clouds without introduction of any label (Figure 3-5). Although isotopic imaging displayed a heterogeneous signal, as the resolution of the technique is lower in comparison to STED and the ${ }^{14} \mathrm{~N}$ signal is more much more dense and widespread, the clouds were not as clearly visible as in fluorescence.

An apparent problem is that not all possible that the fluorophore will not be able to reach and react with all the AHA sites, depending on the position. For example, it is quite likely that residues in the hydrophobic segments transversing the bilayer are not all that accessible for the click reaction. This could lead to fewer targets to be imaged than possible. However, this is a problem for all possible methods of secondary labeling and currently there is no alternative method to avoid it. Unfortunately, a primary labeling method, where all proteins will be fluorescently labeled right away is not feasible for indiscriminate labeling. This is also another reason that SIMS imaging without additional labeling or tagging was necessary. 
Yet another issue with fluorophores is their photophysical interactions with each other. When clustered at high densities, some fluorophores tend to undergo self-quenching, giving decreased emission. For the fluorophores I have used in cloud labeling, Atto647N and Chromeo494, the quenching characteristics are not known, therefore it is not possible to predict the effect of selfquenching phenomenon to our brightness-dependent estimations of proteins enrichment in clouds. Due to this, the calculated enrichment values are potentially underestimated and should be taken as indications rather than absolute quantifications.

\subsubsection{Fluorescent Lipid Analogues}

There is always an inherent problem with fluorescent imaging of lipids, as addition of a fluorophore could alter the properties and interactions of the lipid in question. Therefore the results obtained are heavily dependent on the type of modification to the lipid. Here, I have used analogues with different modifications, acyl chain or headgroup labeling with Atto647N or addition of KK114 to the headgroup through a PEG linker. In many cases, headgroup labeling preserves the properties of lipids better than acyl chain labeling (Sezgin et al., 2012b). The lipids with Atto647N headgroup labeling have been previously used in STED-FCS measurements in living cells. It was found that SM-Atto647N is trapped in small areas with diameter $<20 \mathrm{~nm}$, whereas PEAtto647N was diffusing freely (Eggeling et al., 2009). Cholesterol depletion was found to reduce the trapping of SM-Atto647N, while it did have an effect on the free diffusion of PE-Atto647N (Eggeling et al., 2009; Mueller et al., 2011). Employing the same chemistries gave me the chance to compare their nanoscale diffusion behavior in living cells to the cloud distribution in membrane sheets. SM-Atto647N was found to be slightly enriched in the cloud centers while PE-Atto647N did not show enrichment in the clouds (Figure 3-8). The cloud dispersion I see after cholesterol depletion (Figure 3-14) is also in accordance with the loss of trapping observed in previous STEDFCS studies (Eggeling et al., 2009; Mueller et al., 2011). Accordingly, it seems like there is a correlation between the cloud enrichment and trapping. This was further tested by using another PEGylated analogue, SM-PEG-KK114. This molecule also does not display trapping (personal communication, Alf Honigmann) and interestingly it was not enriched in the clouds either.

Although this correlation between trapping and cloud enrichment is noteworthy, the mechanism behind it is not completely understood. The trapping is expected to occur due to presence of short-lived and cholesterol-assisted molecular complexes (Mueller et al., 2011). Also the relevance of the observed trapping behavior to the native lipids is not clear, since different analogues yield different phase preferences. SM-Atto647N and PE-Atto647N strongly prefer the $L_{d}$ 
phase in GUVs and GPMVs (Sezgin et al., 2012b and personal communication, Alf Honigmann). The addition of a PEG linker, which keeps the fluorophore away from the bilayer, and use of a less hydrophobic KK114 dye makes SM-PEG-KK114 more $L_{d}$ preferring (personal communication, Alf Honigmann), whereas Chol-PEG-KK114 does not have a significant preference for either phase (Figure 2-2). Strikingly, neither the trapping behavior nor the cloud enrichment indicates a correlation with the respective $L_{0} / L_{d}$ partitioning of the lipid analogues in GUVs or GPMVs (Sezgin et al., 2012b). Both the partitioning and the trapping behavior of lipid analogs seems to be determined by a complex combination of a multitude of factors, such as the polarity, size and charge of the label, the label position and headgroup size of the lipid and its interactions with proteins (Sezgin et al., 2012b). Thus, the physicochemical basis for trapping in living cells is yet to be identified, but potentially there could be a link between trapping of lipid analogues and their slight enrichment at the cloud centers.

Interestingly, I have observed that the cholesterol analogue, Chol-PEG-KK114, was not enriched in the clouds, and was actually slightly excluded from the cloud centers (Figure 3-8A). The initial characterization of Chol-PEG-KK114 in GUVs showed promising results with an equal preference for Lo and Ld phases (Figure 2-2). Yet, like any other cholesterol analogue, it is possible that CholPEG-KK114 might not behave identical to native cholesterol in cellular membranes. Therefore the contribution of the linker and dye to this exclusion behavior is at the moment not very clear. Nevertheless, it is likely that cholesterol, is not specifically enriched in the clouds and assumes a more homogeneous distribution, in line with recent isotopic imaging results (Frisz et al., 2013a). Collectively, the profiles of lipid analogs indicate that unlike many different proteins none of the major lipid species (PC, SM and cholesterol) has a tendency to be substantially enriched in the clouds (Figure 3-8A).

\subsubsection{Imaging}

It is apparent that conventional light microscopy would not yield enough resolution for this kind of a study, which aims to distinguish small domains in a densely labeled sample. For imaging the distribution of proteins I have employed STED microscopy. There are also other high-resolution alternatives, which can potentially give even better resolution, such as localization microscopy techniques. However, these techniques require heavy post-processing and can introduce imaging artifacts for analysis of clusters. Localization microscopy methods depend on stochastic switching of fluorophores and stochasticity can introduce potential artifacts like under-sampling or oversampling of the fluorophores (Annibale et al., 2011). Especially over-sampling is an important 
problem in studies of clustering. Reversible cycling of the fluorophores between activated and dark states can cause a fluorophore to be counted more than once. In this case over-counting leads to images with artifactual self-clustering (Veatch et al., 2012).

Since the clouds are expected to be rather densely labeled, it is also possible that stochastically collected signal will not correspond to individual emitters decreasing the apparent resolution of these techniques. Also the differences in the molecular orientation of the fluorophore (which could be quite various for co-labeling of a broad range of targets like in here) can adversely affect the accuracy of centroid calculation for localization again reducing the resolution (Engelhardt et al., 2011). Similarly, fluorophore switching might be highly affected by the density of molecules surrounding the probe in the microdomain. Therefore, although localization microscopy can theoretically offer better resolution, for the particular labeling situation here, STED microscopy was considered more reliable. The ease of imaging, availability of the fluorophores and direct access to the instrument were other factors that made STED more preferable.

There is, however, one type of artifact that STED can create. Since the emission is detected by point scanning of the sample, it is in theory possible that the partial bleaching introduced while scanning can cause a domain-like appearance in the image. Practically, this is not expected to influence our results since the same areas can be imaged repeatedly without visible changes in the resulting pattern.

\subsubsection{Probing Specific Protein Species}

For any clustering study, it is important to be aware of the possible artifacts created by the probes. When possible, it is of course desirable to use monovalent probes to avoid probe-induced clustering. However, these probes might not be available for all targets. Unfortunately, for most proteins I had to rely on antibodies. The antibodies were applied onto sheets after a 45-minute PFA fixation step and the click reaction. Although a mixture of PFA and glutaraldehyde is shown to restrict the mobility better than PFA itself, it is not always possible to use this condition due to loss of antigenicity. To keep the conditions same between different proteins, distributions were mainly investigated in PFA-fixed samples. But as controls some of the stainings were also reproduced in whole cells PFA and glutaraldehyde fixed with PFA and glutaraldehyde. Additionally, small monovalent probes, such as directly labeled Fab fragments (syntaxin 1), RNA aptamers (TfnR) or $\alpha$-bungarotoxin (acetylcholine receptor) were used to confirm the distributions. 


\subsubsection{Limitations Related to Membrane Sheets}

I hypothesize here that a cholesterol-controlled, actin-anchored protein cloud distribution is a fundamental characteristic of the plasma membrane. However, this model is largely derived from membrane sheets, although most of the key results were also verified in whole cells, both fixed and living. The membrane sheets are representative of non-artificial plasma membranes, but may not be identical to the membranes of living cells.

Without doubt, the dynamics of the living cell in the absence of the equilibrium state for many components may deviate the direct application of our model to living cells (Mouritsen, 2011). For example, plasma membrane heterogeneity was modeled based on lateral diffusion and membrane trafficking. The model predicted that persistent and dynamic protein patches would be formed and maintained by barriers to lateral diffusion and vesicle traffic (Gheber and Edidin, 1999). In membrane sheets, the exchange of material with the rest of the cell is blocked upon sonication, which may lead to the stabilization of protein and lipid domains that were more transient, and therefore less evident, in living cells. Another important problem with the equilibrium state is the potential changes in the transbilayer distributions of fast flip-flopping lipids like cholesterol. It is hard to estimate how the bilayer distributions of lipids change in the membrane sheets compared to the living cells.

A recent model also proposed a major role for active flows of actin to regulate dynamic of clustering behavior of the proteins (Gowrishankar et al., 2012). Using fixed membrane sheets it is of course not possible to reflect the dynamic regulation of the protein domains. Immunostaining of the sheets against cytoskeletal elements like actin displays the presence of cytoskeleton attached to the membrane. However, due to the sonication procedure, it is expected that there might be a partial loss of the cortical cytoskeleton.

Several lines of evidence argue that these difficulties are unlikely to have perturbed our results in too strong a fashion. First, protein clouds similar to those from membrane sheets were observed on the plasma membranes of non-sonicated, normal cells (Figure 3-4 and Figure 3-5). Second, membrane structures resembling the protein clouds, and influencing protein and lipid diffusion in the manner expected from protein clouds, were noted in living cells (Figure 3-9 and Figure 3-17). Third, although the membrane sheets lack some of the mechanisms taking place in living cells (such as the continual enzymatic modification of lipids), they still show the typical characteristics of plasma membranes, including unchanged diffusion (Sieber et al., 2007) and may represent an 
equilibrium state that is uncoupled from the cellular dynamics. Finally, the membrane sheets, unlike many other model membranes, maintain the complex composition of cellular membranes, while they are much easier to manipulate than whole cells. The ease of manipulation is actually important to assay and compare the contribution of different factors, because the perturbations that I apply cannot be compensated as would be in living cells. This enables more effective perturbations and to seize the more inherent properties of the plasma membrane. On a different note, all of the limitations listed are also present in other alternative systems such as GPMVs or plasma membrane spheres.

Undeniably, the mechanisms patterning membranes in living cells are considerably more complex. For example, the clouds would be constantly modified by their interactions with various cellular components of the living cell. In that regard, my results present a snapshot view of the expectedly rather dynamic protein organization in the membrane

\subsubsection{Limitations Related to Treatments}

To dissect the effect of different factors, I have applied several treatments to the cells (cytoskeleton disruption, ionomycin) or to the sheets (different ionic conditions, phospholipases, sphingomyelinase, cholesterol depletion). Expectedly, these treatments might have side effects on other components of the membrane. For example, peripheral membrane proteins typically associate with membranes through electrostatic interactions (Singer, 1974). These proteins can be extracted from membranes under conditions of high or low ionic strength. Similarly, treatments on lipids can cause loss of proteins in direct relation to these lipids. Nevertheless, these side effects were not as strong to alter the general patterning behavior.

The global cholesterol effect shown here suggests that cholesterol depletion would have effects on proteins that are not in direct interaction with cholesterol. This has been indeed the case for many proteins (Harder et al., 1998; Murray and Tamm, 2009). However, there are previous studies that imply some of these results may be due to side effects of CD rather than a cholesterol-dependent outcome, because these effects were not reproduced under some cases of metabolic cholesterol depletion (Shvartsman et al., 2006). Part of these differences were attributed to potential influences of CDs on metabolic and signaling pathways (Kenworthy, 2008; Shvartsman et al., 2006). Although it is quite possible that there are associated side effects, it should also be expected that the outcome of an acute treatment like with MBCD might not be the same as a long-term metabolic depletion. Also it should be kept in mind that on whole cells MBCD 
action is limited to the outer leaflet of the plasma membrane whereas the metabolic reduction of cholesterol has overall consequences on the cell. Nevertheless, I think our results can be claimed to be largely cholesterol-related based on a few reasons. Firstly, since the treatments were done on sheets rather than whole cells, there is little risk of inducing side effects through signaling reactions or metabolic changes. Secondly, we can reproduce the same effect with two different $\beta$ CDs and with COase and not with $\alpha$-cyclodextrin that cannot extract cholesterol (Ohtani et al., 1989; Zidovetzki, 2007). More importantly, replenishing cholesterol was able to reverse the MBCD phenotype, so these controls suggest that the results are not governed by an unrelated side effect of $\mathrm{MBCD}$ but are really dependent on cholesterol depletion.

Another issue is regarding the actin. Cholesterol depletion in cells is known to cause a decrease in plasma membrane levels of PIP2 which is involved in regulation of cytoskeleton (Kwik et al., 2003). Using membrane sheets instead of cells should in principle avoid this kind of signaling-dependent side effects of cholesterol depletion. Nonetheless, an inherent problem with the membrane sheets is the possible partial loss of the cortical cytoskeleton during the sonication. Due to this problem, I cannot neglect the possibility that loss of some of the actin cytoskeleton might leave the membrane sheet more open to the action of cholesterol depletion. It might be that in living cells having a tighter cytoskeleton barrier reduces the extent of the cloud dispersion by cholesterol depletion. However, in living cell depletion experiments PIP2-dependent indirect loss of cytoskeleton comes into play (Kwik et al., 2003), negating the advantage.

\subsubsection{Conceptual Caveats}

From a conceptual point of view, it is important to note that our line scans assume a circular 2D geometry for simplicity. However, most clouds have quite complex shapes and are not strictly circular. This of course introduces a bias to all of the line scan based distribution calculations. Nevertheless, it should be noted that the profiles presented are all obtained by averaging of the many domains in many membrane sheets and should not be taken as absolute localizations. Instead, they aim to show general tendencies of different proteins and subsets of proteins. On single protein level, it is highly likely that clouds of different properties exist. The distribution for specific proteins within particular clouds can be different than other clouds or could be temporally regulated depending on the conditions of the cell. Our model just presents a snapshot of the averaged tendencies. Therefore for example seeing TfnR at the edges should not mean that in every cloud, TfnR would be found at the edge. There are actually many clouds that do not have TfnR signal at all. Also TfnR that is already taken into a coated pit at the verge of endocytosis can 
expectedly have a different distribution than the molecule just carried back to the membrane. The same principle is valid for all the molecules. It is entirely possible that there are clouds with only a subset of proteins, for example caveolae-like assemblies. Likewise, clouds or regions of clouds) that have higher cholesterol enrichment might be richer in cholesterol or sphingomyelinpreferring proteins creating rafts. Actually keeping proteins in different regions of the same cloud or in separate nearby clouds and reorganizing the clouds to bring the interacting partners together in response to a signal can be a means to mediate signaling cascades ready to fire efficiently but also well-controlled and temporally regulated. This possibility has already been explored for T-cell antigen receptor (TCR) complexes and linker for activation of T cells (Lat) in the case of protein islands (Lillemeier et al., 2010). These two proteins were found to localize in different protein domains (islands) at the resting state but were co-localized in the same domain after antigen recognition.

Another point is that the general consequences of treatments such as changes in ionic conditions, actin disruption or cholesterol depletion can be different than their effects on single proteins. Depending on their specific interactions, proteins might be more or less susceptible to certain treatments than average. For example, proteins that prefer to be close to cholesterol might be more sensitive to small decreases in cholesterol levels, whereas a substantial depletion might be necessary for others. It is likely that even under the depletion conditions presented in Figure 3-14 there are still some small persisting clusters.

In these assays I have tackled the membrane organization largely from a protein-based perspective. However, in parallel to proteins, domains of different lipids can also be present in the membrane and might be important for the cloud pattern. We were able to address the lipid organization only through incorporation of fluorescent lipid analogues that might not reflect the native situation in the cells. Yet, identification of the lipid domains in native state is technically very challenging and poses an unresolved issue in the field.

\subsection{Compatibility of Protein Clouds with Previous Models}

Though it might include some conceptual differences, the protein cloud hypothesis agrees to some extent with main principles of several prominent membrane-structuring models, including the separation of protein domains by actin fences (Kusumi and Sako, 1996; Ritchie et al., 2003), membrane rafts (Lingwood and Simons, 2010; Simons and Ikonen, 1997) or protein islands (Lillemeier et al., 2006). 


\subsubsection{Membrane Skeleton and Diffusion Measurements}

Immunostaining of sheets against actin revealed that actin does border the clouds in agreement with the actin fence hypothesis and previous actin imaging results (Frankel et al., 2006; Morone et al., 2006; Ritchie et al., 2003). Our antibody-based imaging conditions does not always allow capturing continuous actin filaments, as antibodies are just sampling the targets, giving a more spotty appearance (Opazo et al., 2012), nevertheless the overall continuity of the larger filaments can be clearly seen (Figure 3-13).

The STED-FCS measurements in living cells demonstrated that the diffusion is $\sim 2$-fold slower in the clouds for proteins and also slightly slowed down for lipids (Figure 3-9). These results are in well accordance with previous single-particle tracking experiments (Dietrich et al., 2002; Fujiwara et al., 2002). I have shown that actin disruption of actin causes coalescence of clouds together in the sheets (Figure 3-12). Also, in experiments with fibroblasts cells, 2 times bigger compartments have been demonstrated in accordance with our the results (Dietrich et al., 2002; Fujiwara et al., 2002; Gudheti et al., 2013).

An important characteristic of membrane patterning has been the "hop diffusion" behavior proposed for both proteins and lipids: the diffusion appears to be confined within membrane compartments bordered transmembrane pickets and actin fences, but occasionally "hop" from one compartment to another (Ritchie et al., 2003). At least for proteins this diffusion behavior could also partly result from the confinement of proteins into the cholesterol-induced protein clouds. The hopping would occur from one cloud to another with faster diffusion in the proteinpoor regions between the clouds.

In summary our results do not contradict with majority of the actin-based data and proposed models. However, we have not observed the membrane skeleton to be the main or only organizer of membrane domains (Kusumi et al., 2011). Actin does not seem to stabilize the clouds as majorly as cholesterol, since its disruption does not lead to dispersion of the clouds (Figure 3-11). From a functional point of view, it is not surprising that actin has a less prominent role than cholesterol: actin is more easily modulated in response to cellular needs (via a plethora of actinmodifying pathways), and is thus a dynamic membrane organizer which may not be used to maintain protein clouds in the long term. 
When the more dynamic models of cortical actin-dependent organization is considered (Goswami et al., 2008; Gowrishankar et al., 2012), our results cannot be considered contradictory or confirming, as we did not have the experimental conditions to concentrate on the dynamic measurements. Yet, from a theoretical point of view, the clouds can be the media where the actions of static actin meshwork and the dynamic filaments meet. The clouds could act as sites where the active proteins influencing the dynamic actin filaments are gathered, and these can in turn organize the more passive proteins within the clouds (Gowrishankar et al., 2012).

\subsubsection{Membrane Rafts}

One of the most important limitations is that we do not know the lipid side of the cloud organization. Our lipid information is limited to the fluorescent lipid analogues and the contribution of lipid domains or phases to the cloud organization is not specifically assayed by our methods. Different fluorescent analogues of with varying $L_{o}$ preferences were applied to the membrane sheets yielded largely homogeneous stainings with no apparent large-scale phase separation. The only difference was in the small-scale enrichment or exclusion of these lipids at the cloud centers, which at this point did not produce a conclusive correlation with their partitioning properties (Figure 3-8, see also Section 4.3.3). Therefore it is hard to comment on the $L_{0} / L_{d}$ distribution of the clouds. Nonetheless, distribution of specific proteins within the clouds can provide some insights.

Raft-preferring proteins, such as the bulk of the palmitoylated and myristoylated proteins (Levental et al., 2010b; Melkonian et al., 1999) appear to occupy cloud centers (Figure 3-7), while the non-raft preferring proteins like the bulk of farnesylated proteins (Melkonian et al., 1999) or TfnR (Mañes et al., 1999) and several proteins known not to be enriched in DRMs, like syntaxin 1 and SNAP25 (Lang et al., 2001; Lang, 2007) share an area at the cloud edges (Figure 3-18). This suggests that some cloud centers may form membrane rafts - or that they may be the underlining structure upon which raft-like or non-raft-like domains are built.

The effect of palmitoylation for increased center preference has been indicated also by the depalmitoylation experiments (Figure 3-23). Two structurally very similar proteins, SNAP25 and SNAP23 were found to have different positioning in the clouds (Figure 3-18). One difference between them is the extent of palmitoylation, SNAP23 has an extra cysteine, which was shown to be important for its higher DRM association (Salaun et al., 2005b). Although application of DTT to the cells to induce removal of palmitoyl moieties (Levental et al., 2010b) caused a some loss of 
SNAP23 and SNAP25, a substantial amount of these lipid-anchored proteins were still attached to the membrane (Figure 3-23B,D). This can either be due to a partial depalmitoylation or to the association of proteins with the membrane even at the absence of lipid anchors, as have been shown for deacylated SNAP25 previously (Gonzalo and Linder, 1998). In any case, loss of palmitoyls caused a slightly broader distribution of the proteins with respect to the clouds. This is also fitting with the role of palmitoylation driving proteins to rafts (Levental et al., 2010b; 2010a).

Generally, detergent resistance of the raft lipids thought to be arising from the tight order of cholesterol and sphingomyelin in the $L_{0}$ phase (Ahmed et al., 1997; London and Brown, 2000). When the complexity of the membrane and the high abundance of the proteins are considered, one can imagine high protein densities partially protecting the lipids from detergent extraction as well. With this perspective, the cloud centers might provide such a protection to the lipids, contributing detergent-resistance. Although it is quite speculative, this can be one explanation for a possible link between the clouds and the DRMs, in the sense that at least some of the DRMs originate from cloud centers. This explanation could also justify the broad spectrum of unrelated proteins found in DRMs (Foster et al., 2003) and the absence of mitochondrial proteins (which do not seem to be gathered in clouds) in DRMs (Zheng et al., 2008)as well as creation of DRMs by flotillin-like proteins in bacterial membrane without cholesterol (López and Kolter, 2010).

When it comes to functional, dynamic membrane rafts in living cells (Pike, 2006), small raft domains might form at various positions within the clouds, possibly through short-lived, transient changes in the local concentration of cholesterol and sphingomyelin, which would be induced by interactions with specific proteins. The strict dependence of clouds upon cholesterol also explains a puzzling observation related to the raft model, namely the disruptive effects of cholesterol depletion on the distribution of proteins that have only a minor dependence on detergentresistant membranes and rafts, such as syntaxin 1, SNAP25 (Lang et al., 2001; Lang, 2007) or TfnR (Harder et al., 1998; Mañes et al., 1999; Quincke, 1888; 1893). Although their clustering is mostly produced through raft and $\mathrm{L}_{0}$-independent interactions their distribution will still be affected by the disturbance caused to the overall membrane cloud pattern by cholesterol removal. Taken together, our results indicate that cholesterol has an additional fundamental function besides the specific interactions with proteins and certain lipids, namely organizing the majority of proteins into protein-dense clouds. 


\subsubsection{Specific Protein Clusters}

Many cases of specialized protein domains are known. Some examples are syntaxin 1 clusters formed by self-assembly (Sieber et al., 2007) or specific interactions with PIP2 (van den Bogaart et al., 2011), cholesterol-mediated caveolae (Rothberg et al., 1990), clusters of lipid-anchored Ras proteins, clathrin-coated pits, calcium-dependent clustering of exocytotic proteins (Zilly et al., 2011) or signaling microdomains of T-cells under activation conditions (Douglass and Vale, 2005). The protein cloud model is in agreement with the formation of such specialized domains within the clouds via (homo or hetero) protein-protein or protein-lipid interactions. Through enrichment of individual proteins together in the clouds, the basic pattern is created for these more specific and functional interactions to take place. This also provides an explanation for the differential enrichment preferences of different proteins within the clouds and the size and shape heterogeneity of the clouds. Depending on the strength of protein-protein interactions, the affinity of a protein species for particular clouds might be variable and also regulated through signaling cascades or post-translational modifications.

When clusters of individual protein species are considered to be components of the clouds, they are expected to reflect the general cloud behavior up to some extent and that is very often the case. For example, small clusters of fluorescently-labeled GPI anchored proteins, which cannot be distinguished with confocal microscopy, were detected in living cells by measurements of fluorescence anisotropy (Goswami et al., 2008). Or cell surface cadherin was probed by molecular recognition imaging by AFM (Müller et al., 2009). Interestingly, the anisotropy pattern or the molecular recognition map seen in the flat parts of the membrane is visually very similar to our cloud pattern. Also a decrease in anisotropy was detected after cholesterol depletion (Goswami et al., 2008), suggesting decreased clustering. Another example is the distribution of hemagglutinin (HA) molecules in living fibroblasts imaged by high-resolution FPALM (Hess et al., 2007). HA was found to form irregular clusters with a wide range of size and shapes and displayed constrained motion, avoiding some areas and mapping out regions with elongated shapes and irregular boundaries. In a follow-up study actin cytoskeleton was found to be important for cluster size and the number of molecules per cluster (Gudheti et al., 2013). The dependence of HA clustering on cholesterol has also been shown before (Eisenberg et al., 2006; Scolari et al., 2009). On a different note, two lipid-anchored signaling proteins K-Ras and H-Ras was shown to have restricted mobility in FRAP experiments (Niv et al., 2002). However, increasing their expression level caused higher mobility suggesting that the domains they localize to are saturable sites, 
which can only accommodate a certain amount of protein. These results are in accordance with the spill-over effect of overexpression shown in Figure 3-10.

To assess the contribution of different factors to the general cloud organization, we have also applied commonly utilized perturbation methods like cholesterol depletion, sphingomyelinase treatment or actin-disrupting drugs. In a recent study similar perturbations were recently applied on living COS-7 cells expressing GPI-anchored photoactivatable GFP (PAGFP-GPI), followed by fixation of cells and high-resolution PALM imaging (Sengupta et al., 2011). Using pair-correlation analysis to overcome the potential artifacts of single molecule localization in cluster identification, parameters such as cluster size, density and number of proteins per cluster were calculated. The clusters investigated in this study are $<60 \mathrm{~nm}$ assemblies of 2-3 proteins. They are apparently not identical to the clouds we see. However, these small clusters could well be part of the clouds together with clusters of other single proteins. The analysis showed that $60 \%$ of the PAGFP-GPI clusters were lost with cholesterol depletion, whereas cholesterol addition resulted in more clusters. Sphingomyelinase and cytochalasin B treatments caused loss of small clusters and led to larger domains (Sengupta et al., 2011). The interesting outcome is that the major consequences of the perturbations on these small PAGFP-GPI clusters under different sample and imaging conditions overlap quite well with our results for general protein labeling.

\subsubsection{General Multi-Protein Domains}

The observation of clouds is not the first time that multi-protein domains are detected in the plasma membrane. Although studies that target general pools of proteins, rather than one specific species, are rare, such protein enrichments have been observed under diverse experimental conditions with different samples.

Stable protein-rich domains surrounded by protein-poor regions in living fibroblasts have been first shown in 1987 (Yechiel and Edidin, 1987), by applying FRAP with different beam sizes. The underlying cause was suggested to be lipid domains stabilized by interactions with proteins. In another study the lateral diffusion coefficients of GFP-tagged proteins of different topologies (bitopic, polytopic, GPI-anchored and palmitoylated proteins) were measured by more modern methods of FRAP in COS-7 cells (Frick et al., 2007). The observed slow diffusion of proteins in cell membrane was mediated by protein density, and not by cortical cytoskeleton. TIRF-based fluorescent tracking of GFP-tagged signaling proteins Lat and Lck also identified trapping of these 
proteins in protein domains defined by presence of $\mathrm{CD} 2$ receptor, in an protein-protein interaction dependent manner (Douglass and Vale, 2005).

In EM experiments performed on membrane sheets from mast cells and T-cells, dark osmiumlabeled patches, which contained a large amount of proteins including signaling factors or raft and non-raft markers, were detected (Lillemeier et al., 2006; Wilson et al., 2004). By AFM topography imaging these protein-rich patches on membranes were identified as raised domains of irregular shapes (Frankel et al., 2006). They were cholesterol-sensitive and were surrounded and linked by the cytoskeleton. Although there are some technical difficulties (EM processing of membrane sheets which might result in extraction of some of the lipids or improper fixation conditions which could create artificial crosslinking) in these previous experiments, in principle, the domains or islands identified might share the same origin with the clouds or might be derivations of them under different experimental conditions and cell types.

Recently, a systematic high-resolution imaging study of GFP coupled membrane proteins in living yeast cells reported that all of the $>40$ proteins investigated were distributed non-homogeneously, forming various patterns of patches and continuous networks (Spira et al., 2012). Interestingly, actin disruption had minor effects and depletion of sphingolipids or PIP2 affected only a subset of proteins, while modifications to sterol composition influenced the distribution of all the proteins. The compatibility of our results with these observations suggests an extension of the identified yeast domains to mammalian cell membranes.

I think that although there could be some condition-dependent alterations, the protein clouds can be principally similar to previously observed protein-rich domains. It is likely that these domains are derivatives of the clouds in other cell types; some of these domains can be in a functionally specialized state or aggregated due to preparation conditions. Cloud model, in a way, presents a united framework for these separate observations. 


\section{SUMMARY AND CONCLUSIONS}

In this work, I studied the distribution of a representative population of all membrane proteins by high-resolution microscopy. I observed that the proteins in the plasma membrane-derived sheets are concentrated in heterogeneous multi-protein domains. Labyrinth-like protein-poor regions frame these high protein abundance domains, forming a mosaic. The protein clouds occupied about slightly more than half of the membrane surface, and contained $81 \%$ of the membrane proteins (Section 3.2 and Figure 3-3). The formation and maintenance of clouds is largely controlled by cholesterol in an unspecific manner. Actin cytoskeleton seems to have a complementary role. It surrounds the clouds and prevents their coalescence, limiting their size. It could also be providing a dynamic aspect to the clouds. In living cells, STED-FCS experiments demonstrated that both the diffusion of proteins and lipids seem to be slowed down in the clouds suggesting that the molecular crowding in the clouds affects the free diffusion through or within them.

If I extend these results to the general organization of the membrane, the protein clouds appear to represent a basic, low hierarchy principle of patterning. For a visual impression, Figure 5-1 illustrates a simplified view of the cloud organization model in the plasma membrane. The clouds concentrate proteins together, creating platforms upon which more specific and functional interactions can act. The differential localizations of specific protein species and the functional involvement of proteins preferring similar sub-cloud regions indicate formation of functional domains within the clouds. Specific protein-protein and protein-lipid interactions are expected to be contributing to this higher level or organization within the clouds. Chemical modifications of the proteins (like palmitoylation or farnesylation) or their interactions with lipid microdomains might also be important for preference of certain sites; however, further investigations are necessary to have a better understanding of these interactions. 


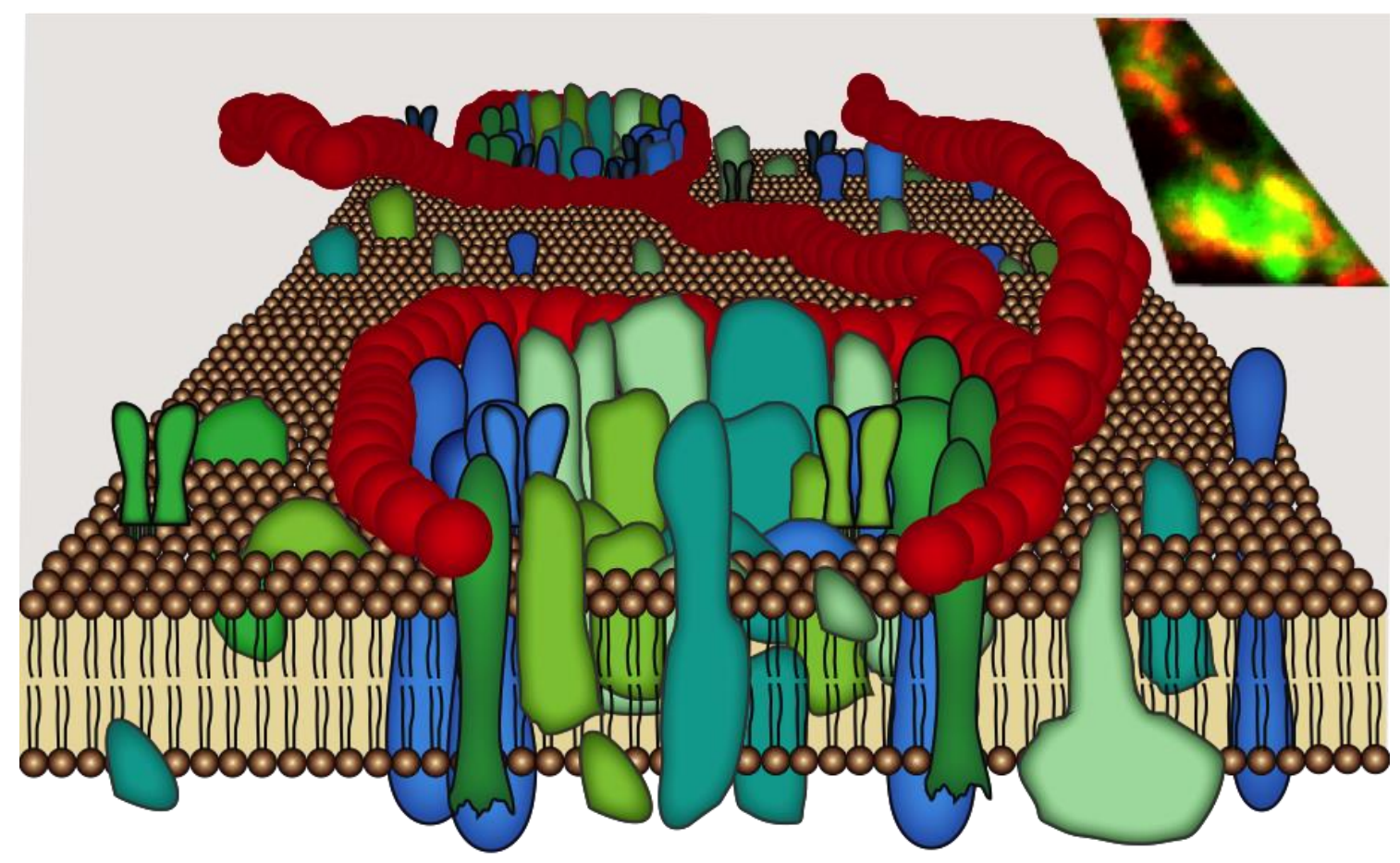

Figure 5-1 Protein clouds as a membrane organizing principle.

The graphics display the representation of the cloud organization in the plasma membrane. Different protein groups are shown in shades of blue and green, and actin is in red. The lipid bilayer is simplified without depiction of different lipid species. The image on the right corner is a crop from a 2-color STED image with click labeling of clouds (green) and immunostaining against actin (red).

As discussed above, many general mechanisms of protein localization have been proposed in the past. Although there might be some discrepancies in the details and immediate outcomes, our model is not directly contradicting any of them in general. Instead, the cloud model is, in a way, unifying the major strengths of the previous models, and presenting the backbone for the main effectors of these models, such as actin fences, rafts, density of proteins, electrostatic interactions and specific protein-protein and protein-lipid interactions to act on. A hierarchical perspective of membrane organization has been suggested before (Kusumi et al., 2011). Formation of membrane skeleton confined compartments was accepted as the first level, followed by formation of lipid rafts, and small, transient assemblies of protein complexes. With the cloud model, a more basic and lower-hierarchy step is added to this organization pyramid. Presence of clouds, as a basic organization principle also clarifies the previously not explained observations, such as effects of cholesterol depletion on non-raft markers and only partial losses in the organization after cytoskeleton disruption. Furthermore by putting the membrane proteins at the center of the membrane organization, the cloud model also brings an alternative explanation for the transbilayer coupling of membrane organization. This has been a controversial aspect of many membrane models where main effectors are supposedly found on one side of the membrane, like 
sphingolipid and cholesterol-rich rafts on the outer leaflet or membrane skeleton associated with the inner leaflet (Devaux and Morris, 2004).

In addition to this new proposal for the general membrane organization, this study presents some important technical implications for future work. The general strategy I have developed here can be used in the future to assay the average distributions of specific proteins. This behavior can be complementary to the standard colocalization experiments. Also, I have shown that simple colocalization information obtained by conventional imaging methods might not always indicate functional partnering since a range of proteins might be sharing similar clouds and diffractionlimited resolution might not be yield enough spatial resolution to distinguish sub-cloud localizations.

A minor result that raises serious concerns about protein overexpression experiments is that the high expression levels of exogenous proteins might cause large-scale redistribution of proteins in the membrane. Furthermore, future studies evaluating the contribution of cholesterol for specific cases through cholesterol depletion methods should ideally take the more general effect of cholesterol into account while interpreting the results.

A major technical contribution is the development of the COIN scheme. The combination of fluorescence labeling/high-resolution imaging with isotopic labeling/mass spectrometry imaging will open several new lines of research. The possible applications of COIN can vary from investigations of material turnover for specific organelles to tag-free imaging of cellular components in different subcellular compartments. 


\section{OUTLOOK}

From a technical point of view it would be ideal to perform follow-up experiments with improved preservation conditions. One way to get more reliable snapshots of the plasma membrane would be to reproduce the experiment by coupling sonication to high-pressure freezing and cryosubstitution (Tokuyasu, 1973; van Donselaar et al., 2007) followed by click labeling. However, these methodology might be technically very challenging and could also be prone to other artifacts like partial extraction of lipids (Möbius et al., 2002). An alternative line would be to get a dynamic view by doing more elaborate experiments in living cells. For this it would be necessary to establish the copper-free click reaction in living cells. I have already tried this strategy but could not get reliable results under the low efficiency and specificity of the labeling with currently available chemical moieties. However, new chemistries are developed at a high pace, so it might be possible to collect more direct information about the cloud organization in living cells.

For whole cell imaging, it is also necessary to use a more complex 3D high-resolution technique like a combination of TIRF and SIM or iso-STED, which is a 3D implementation of the STED technique. Additionally, stainings for specific proteins can be improved by more comprehensive use of smaller, monovalent probes such as directly-labeled nanobodies rather than antibody complexes. These tools now are being developed in our group. It would also be ideal to reproduce the STED-FCS results with monovalent probes.

In order to obtain information about the dynamics of single protein and lipid species, the diffusion of different groups of lipids and proteins can be systematically analyzed by complementary STEDFCS and SPT measurements. From another perspective, a more comprehensive COIN imaging can be performed for the surfaces of whole cells, and protein clouds and different lipids can be visualized together by incorporation of native lipids and amino acids containing stable isotopes. The technical challenge here is to reach high enough resolution while keeping signal-to-noise ratio at a reasonable level.

To add a more solid lipid angle to the model and to understand the role of membrane order/fluidity, emerging imaging modalities such as fluorescence lifetime imaging with environment-sensitive dyes might be performed in parallel with the imaging of clouds. 


\section{BIBLIOGRAPHY}

Abbe, E. (1873). Beiträge zur Theorie des Mikroskops und der mikroskopischen Wahrnehmung. Archiv F Mikrosk Anatomie 9, 413-418.

Abney, J.R., and Scalettar, B.A. (1995). Fluctuations and membrane heterogeneity. Biophys Chem $57,27-36$.

Acehan, D., Malhotra, A., Xu, Y., Ren, M., Stokes, D.L., and Schlame, M. (2011). Cardiolipin Affects the Supramolecular Organization of ATP Synthase in Mitochondria. Biophys J 100, 2184-2192.

Ahmed, S.N., Brown, D.A., and London, E. (1997). On the Origin of Sphingolipid/Cholesterol-Rich Detergent-Insoluble Cell Membranes: Physiological Concentrations of Cholesterol and Sphingolipid Induce Formation of a Detergent-Insoluble, Liquid-Ordered Lipid Phase in Model Membranes. Biochemistry 36, 10944-10953.

Alberts, B., Johnson, A., Lewis, J., Raff, M., Roberts, K., and Walter, P. (2007). Molecular Biology of the Cell (New York: Garland Science).

Ali, M.R., Cheng, K.H., and Huang, J. (2007). Assess the nature of cholesterol-lipid interactions through the chemical potential of cholesterol in phosphatidylcholine bilayers. Proc Natl Acad Sci USA 104, 5372-5377.

Almeida, P.F.F., Pokorny, A., and Hinderliter, A. (2005). Thermodynamics of membrane domains. Biochim Biophys Acta 1720, 1-13.

Anderson, R.G.W. (2002). A Role for Lipid Shells in Targeting Proteins to Caveolae, Rafts, and Other Lipid Domains. Science 296, 1821-1825.

Annibale, P., Vanni, S., Scarselli, M., Rothlisberger, U., and Radenovic, A. (2011). Identification of clustering artifacts in photoactivated localization microscopy. Nature Met 8, 527-528.

Avery, J., Ellis, D.J., Lang, T., Holroyd, P., Riedel, D., Henderson, R.M., Edwardson, J.M., and Jahn, R. (2000). A cell-free system for regulated exocytosis in PC12 cells. J Cell Biol 148, 317-324.

Baker, J.R. (1952). The Cell-Theory: A Restatement, History, and Critique. Q J Microsc Sci 93, 157190.

Balch, W.E., and Rothman, J.E. (1985). Characterization of protein transport between successive compartments of the Golgi apparatus: asymmetric properties of donor and acceptor activities in a cell-free system. Arch Biochem Biophys 240, 413-425.

Bar, R.S., Deamer, D.W., and Cornwell, D.G. (1966). Surface area of human erythrocyte lipids: reinvestigation of experiments on plasma membrane. Science 153, 1010-1012.

Bar-On, D., Wolter, S., van de Linde, S., Heilemann, M., Nudelman, G., Nachliel, E., Gutman, M., Sauer, M., and Ashery, U. (2012). Super-resolution imaging reveals the internal architecture of 
nano-sized syntaxin clusters. J Biol Chem 287, 27158-27167.

Barysch, S.V., Aggarwal, S., Jahn, R., and Rizzoli, S.O. (2009). Sorting in early endosomes reveals connections to docking- and fusion-associated factors. Proc.Natl Acad Sci USA 106, 9697-9702.

Barysch, S.V., Jahn, R., and Rizzoli, S.O. (2010). A fluorescence-based in vitro assay for investigating early endosome dynamics. Nat Protoc 5, 1127-1137.

Baumgart, T., Hammond, A.T., Sengupta, P., Hess, S.T., Holowka, D.A., Baird, B.A., and Webb, W.W. (2007). Large-scale fluid/fluid phase separation of proteins and lipids in giant plasma membrane vesicles. Proc Natl Acad Sci USA 104, 3165-3170.

Bennett, W.F.D., MacCallum, J.L., Hinner, M.J., Marrink, S.J., and Tieleman, D.P. (2009). Molecular view of cholesterol flip-flop and chemical potential in different membrane environments. J Am Chem Soc 131, 12714-12720.

Benson, A.A. (1966). On the orientation of lipids in chloroplast and cell membranes. J Am Oil Chem Soc 43, 265-270.

Bethani, I., Werner, A., Kadian, C., Geumann, U., Jahn, R., and Rizzoli, S.O. (2009). Endosomal Fusion upon SNARE Knockdown is Maintained by Residual SNARE Activity and Enhanced Docking. Traffic 10, 1543-1559.

Betzig, E., Patterson, G.H., Sougrat, R., Lindwasser, O.W., Olenych, S., Bonifacino, J.S., Davidson, M.W., Lippincott-Schwartz, J., and Hess, H.F. (2006). Imaging Intracellular Fluorescent Proteins at Nanometer Resolution. Science 313, 1642-1645.

Bhatia, V.K., Madsen, K.L., Bolinger, P.-Y., Kunding, A., Hedegård, P., Gether, U., and Stamou, D. (2009). Amphipathic motifs in BAR domains are essential for membrane curvature sensing. Embo J 28, 3303-3314.

Bhatnagar, R.S., and Gordon, J.I. (1997). Understanding covalent modifications of proteins by lipids: where cell biology and biophysics mingle. Trends Cell Biol 7, 14-20.

Bhattacharyya, R., Barren, C., and Kovacs, D.M. (2013). Palmitoylation of amyloid precursor protein regulates amyloidogenic processing in lipid rafts. J Neurosci 33, 11169-11183.

Blonder, J., Hale, M.L., Lucas, D.A., Schaefer, C.F., Yu, L.-R., Conrads, T.P., Issaq, H.J., Stiles, B.G., and Veenstra, T.D. (2004). Proteomic analysis of detergent-resistant membrane rafts. Electrophoresis 25, 1307-1318.

Boekema, E.J., Scheffers, D.-J., van Bezouwen, L.S., Bolhuis, H., and Folea, I.M. (2013). Focus on membrane differentiation and membrane domains in the prokaryotic cell. J Mol Microbiol Biotechnol 23, 345-356.

Bonanomi, D., Benfenati, F., and Valtorta, F. (2006). Protein sorting in the synaptic vesicle life cycle. Prog Neurobiol 80, 177-217. 
Brown, D.A., and Rose, J.K. (1992). Sorting of GPI-anchored proteins to glycolipid-enriched membrane subdomains during transport to the apical cell surface. Cell 68, 533-544.

Butschli, O. (1892). Untersuchungen über Mikroskopische Schäume und das Protoplasma (Leipzig: Engelmann).

Cahill, A.L., Herring, B.E., and Fox, A.P. (2006). Stable silencing of SNAP-25 in PC12 cells by RNA interference. BMC Neurosci 7, 9.

Cambridge, S.B., Gnad, F., Nguyen, C., Bermejo, J.L., Krüger, M., and Mann, M. (2011). Systemswide proteomic analysis in mammalian cells reveals conserved, functional protein turnover. $J$ Proteome Res 10, 5275-5284.

Canevari, L., and Clark, J.B. (2007). Alzheimer's disease and cholesterol: the fat connection. Neurochem Res 32, 739-750.

Cao, X., Surma, M.A., and Simons, K. (2012). Polarized sorting and trafficking in epithelial cells. Cell Res 22, 793-805.

Castaing, R., and Slodzian, G. (1962). Microanalysis by secondary ionic emission. J Microsc 395410.

Chapman, D. (1975). Phase transitions and fluidity characteristics of lipids and cell membranes. Q Rev Biophys 8, 185-235.

Chazotte, B. (2011). Labeling Membrane Glycoproteins or Glycolipids with Fluorescent Wheat Germ Agglutinin. Cold Spring Harb Protoc 2011, pdb.prot5623-pdb.prot5623.

Cho, W., and Stahelin, R.V. (2005). Membrane-protein interactions in cell signaling and membrane trafficking. Annu Rev Biophys Biomol Struct 34, 119-151.

Christian, A., Haynes, M., and Phillips, M. (1997). Use of cyclodextrins for manipulating cellular cholesterol content. J Lipid Res 38, 2264-2272.

Coskun, U., and Simons, K. (2011). Cell membranes: the lipid perspective. Structure 19, $1543-$ 1548.

Coskun, U., Grzybek, M., Drechsel, D., and Simons, K. (2011). Regulation of human EGF receptor by lipids. Proc Natl Acad Sci USA 108, 9044-9048.

Daleke, D.L. (2007). Phospholipid flippases. J. Biol Chem 282, 821-825.

Daum, G. (1985). Lipids of mitochondria. Biochim Biophys Acta 822, 1-42.

Daumas, F., Destainville, N., Millot, C., Lopez, A., Dean, D., and Salomé, L. (2003). Interprotein interactions are responsible for the confined diffusion of a G-protein-coupled receptor at the cell surface. Biochem Soc Trans 31, 1001-1005.

de Almeida, R.F.M., Fedorov, A., and Prieto, M. (2003). Sphingomyelin/ phosphatidylcholine/ 
cholesterol phase diagram: boundaries and composition of lipid rafts. Biophys J 85, 2406-2416.

de Almeida, R.F.M., Loura, L.M.S., Prieto, M., Watts, A., Fedorov, A., and Barrantes, F.J. (2004). Cholesterol modulates the organization of the gammaM4 transmembrane domain of the muscle nicotinic acetylcholine receptor. Biophys J 86, 2261-2272.

de Meyer, F.J.-M., Rodgers, J.M., Willems, T.F., and Smit, B. (2010). Molecular simulation of the effect of cholesterol on lipid-mediated protein-protein interactions. Biophys J 99, 3629-3638.

Denker, A., Kröhnert, K., and Rizzoli, S.O. (2009). Revisiting synaptic vesicle pool localization in the Drosophila neuromuscular junction. J Physiol 587, 2919-2926.

Destainville, N. (2008). Cluster phases of membrane proteins. Phys Rev E Stat Nonlin Soft Matter Phys 77, 011905.

Destainville, N., Dumas, F., and Salomé, L. (2008a). What do diffusion measurements tell us about membrane compartmentalisation? Emergence of the role of interprotein interactions. J Chem Biol 1, 37-48.

Destainville, N., Saulière, A., and Salomé, L. (2008b). Comment to the article by Michael J. Saxton: A biological interpretation of transient anomalous subdiffusion. I. qualitative model. Biophys J 95, 3117-3119.

Devaux, P.F., and Morris, R. (2004). Transmembrane asymmetry and lateral domains in biological membranes. Traffic 5, 241-246.

Di Paolo, G., and Kim, T.-W. (2011). Linking lipids to Alzheimer's disease: cholesterol and beyond. Nat Rev Neurosci 12, 284-296.

Dieterich, D.C., Hodas, J.J.L., Gouzer, G., Shadrin, I.Y., Ngo, J.T., Triller, A., Tirrell, D.A., and Schuman, E.M. (2010). In situ visualization and dynamics of newly synthesized proteins in rat hippocampal neurons. Nat Neurosci 13, 897-905.

Dieterich, D.C., Lee, J.J., Link, A.J., Graumann, J., Tirrell, D.A., and Schuman, E.M. (2007). Labeling, detection and identification of newly synthesized proteomes with bioorthogonal non-canonical amino-acid tagging. Nat Protoc 2, 532-540.

Dieterich, D.C., Link, A.J., Graumann, J., Tirrell, D.A., and Schuman, E.M. (2006). Selective identification of newly synthesized proteins in mammalian cells using bioorthogonal noncanonical amino acid tagging (BONCAT). Proc Natl Acad Sci USA 103, 9482-9487.

Dietrich, C., Yang, B., Fujiwara, T., Kusumi, A., and Jacobson, K. (2002). Relationship of lipid rafts to transient confinement zones detected by single particle tracking. Biophys J 82, 274-284.

Douglass, A.D., and Vale, R.D. (2005). Single-Molecule Microscopy Reveals Plasma Membrane Microdomains Created by Protein-Protein Networks that Exclude or Trap Signaling Molecules in T Cells. Cell 121, 937-950. 
Dudkina, N.V., Oostergetel, G.T., Lewejohann, D., Braun, H.-P., and Boekema, E.J. (2010). Row-like organization of ATP synthase in intact mitochondria determined by cryo-electron tomography. Biochim Biophys Acta 1797, 272-277.

Dupuy, A.D., and Engelman, D.M. (2008). Protein area occupancy at the center of the red blood cell membrane. Proc Natl Acad Sci USA 105, 2848-2852.

Edidin, M. (2001). Shrinking patches and slippery rafts: scales of domains in the plasma membrane. Trends Cell Biol 11, 492-496.

Edidin, M., and Stroynowski, I. (1991). Differences between the lateral organization of conventional and inositol phospholipid-anchored membrane proteins. A further definition of micrometer scale membrane domains. J Cell Biol 112, 1143-1150.

Edidin, M., Kuo, S.C., and Sheetz, M.P. (1991). Lateral movements of membrane glycoproteins restricted by dynamic cytoplasmic barriers. Science 254, 1379-1382.

Edidin, M. (2003). Lipids on the frontier: a century of cell-membrane bilayers. Nat Rev Mol Cell Biol 4, 414-418.

Eggeling, C., Ringemann, C., Medda, R., Schwarzmann, G., Sandhoff, K., Polyakova, S., Belov, V.N., Hein, B., Middendorff, von, C., Schönle, A., et al. (2009). Direct observation of the nanoscale dynamics of membrane lipids in a living cell. Nature 457, 1159-1162.

Ehehalt, R., Keller, P., Haass, C., Thiele, C., and Simons, K. (2003). Amyloidogenic processing of the Alzheimer beta-amyloid precursor protein depends on lipid rafts. J Cell Biol 160, 113-123.

Eisenberg, S., Shvartsman, D.E., Ehrlich, M., and Henis, Y.I. (2006). Clustering of raft-associated proteins in the external membrane leaflet modulates internal leaflet $\mathrm{H}$-ras diffusion and signaling. Mol Cell Biol 26, 7190-7200.

Engelhardt, J., Keller, J., Hoyer, P., Reuss, M., Staudt, T., and Hell, S.W. (2011). Molecular orientation affects localization accuracy in superresolution far-field fluorescence microscopy. Nano Lett 11, 209-213.

Epand, R.M. (2008). Proteins and cholesterol-rich domains. BBA-Biomembranes 1778, 1576-1582.

Epand, R.M., Sayer, B.G., and Epand, R.F. (2005). Caveolin Scaffolding Region and Cholesterol-rich Domains in Membranes. J Mol Biol 345, 339-350.

Foster, L.J. (2008). Lessons learned from lipid raft proteomics. Expert Rev Proteomics 5, 541-543.

Foster, L.J., De Hoog, C.L., and Mann, M. (2003). Unbiased quantitative proteomics of lipid rafts reveals high specificity for signaling factors. Proc Natl Acad Sci USA 100, 5813-5818.

Frankel, D.J., Pfeiffer, J.R., Surviladze, Z., Johnson, A.E., Oliver, J.M., Wilson, B.S., and Burns, A.R. (2006). Revealing the topography of cellular membrane domains by combined atomic force microscopy/fluorescence imaging. Biophys J 90, 2404-2413. 
Frick, M., Schmidt, K., and Nichols, B.J. (2007). Modulation of lateral diffusion in the plasma membrane by protein density. Curbio 17, 462-467.

Frisz, J.F., Klitzing, H.A., Lou, K., Hutcheon, I.D., Weber, P.K., Zimmerberg, J., and Kraft, M.L. (2013a). Sphingolipid domains in the plasma membranes of fibroblasts are not enriched with cholesterol. J Biol Chem 288, 16855-16861.

Frisz, J.F., Lou, K., Klitzing, H.A., Hanafin, W.P., Lizunov, V., Wilson, R.L., Carpenter, K.J., Kim, R., Hutcheon, I.D., Zimmerberg, J., et al. (2013b). Direct chemical evidence for sphingolipid domains in the plasma membranes of fibroblasts. Proc Natl Acad Sci USA 110, E613-E622.

Frye, L.D., and Edidin, M. (1970). The rapid intermixing of cell surface antigens after formation of mouse-human heterokaryons. J Cell Sci 7, 319-335.

Fujita, A., Cheng, J., Hirakawa, M., Furukawa, K., Kusunoki, S., and Fujimoto, T. (2007). Gangliosides GM1 and GM3 in the Living Cell Membrane Form Clusters Susceptible to Cholesterol Depletion and Chilling. Mol Biol Cell 18, 2112-2122.

Fujita, A., Cheng, J., Tauchi-Sato, K., Takenawa, T., and Fujimoto, T. (2009). A distinct pool of phosphatidylinositol 4, 5-bisphosphate in caveolae revealed by a nanoscale labeling technique. Proc Natl Acad Sci USA 106, 9256-9261.

Fujiwara, T., Ritchie, K., Murakoshi, H., Jacobson, K., and Kusumi, A. (2002). Phospholipids undergo hop diffusion in compartmentalized cell membrane. J Cell Biol 157, 1071-1081.

Gheber, L.A., and Edidin, M. (1999). A model for membrane patchiness: lateral diffusion in the presence of barriers and vesicle traffic. Biophys J 77, 3163-3175.

Gonzalo, S., and Linder, M.E. (1998). SNAP-25 palmitoylation and plasma membrane targeting require a functional secretory pathway. Mol Biol Cell 9, 585-597.

Gorter, E., and Grendel, F. (1925). On Bimolecular Layers of Lipoids on the Chromocytes of the Blood. J Exp Med 41, 439-443.

Goswami, D., Gowrishankar, K., Bilgrami, S., Ghosh, S., Raghupathy, R., Chadda, R., Vishwakarma, R., Rao, M., and Mayor, S. (2008). Nanoclusters of GPI-anchored proteins are formed by cortical actin-driven activity. Cell 135, 1085-1097.

Gowrishankar, K., Ghosh, S., Saha, S., C, R., Mayor, S., and Rao, M. (2012). Active remodeling of cortical actin regulates spatiotemporal organization of cell surface molecules. Cell 149, 1353-1367.

Gudheti, M.V., Curthoys, N.M., Gould, T.J., Kim, D., Gunewardene, M.S., Gabor, K.A., Gosse, J.A., Kim, C.H., Zimmerberg, J., and Hess, S.T. (2013). Actin mediates the nanoscale membrane organization of the clustered membrane protein influenza hemagglutinin. Biophys J 104, 21822192.

Halemani, N.D., Bethani, I., Rizzoli, S.O., and Lang, T. (2010). Structure and Dynamics of a TwoHelix SNARE Complex in Live Cells. Traffic 11, 394-404. 
Halpern, B.C., Clark, B.R., Hardy, D.N., Halpern, R.M., and Smith, R.A. (1974). The effect of replacement of methionine by homocystine on survival of malignant and normal adult mammalian cells in culture. Proc Natl Acad Sci USA 71, 1133-1136.

Hamada, K., Shimizu, T., Matsui, T., Tsukita, S., and Hakoshima, T. (2000). Structural basis of the membrane-targeting and unmasking mechanisms of the radixin FERM domain. Embo J 19, 44494462.

Hancock, J.F., Magee, A.I., Childs, J.E., and Marshall, C.J. (1989). All ras proteins are polyisoprenylated but only some are palmitoylated. Cell 57, 1167-1177.

Hancock, J.F. (2003). Ras proteins: different signals from different locations. Nat Rev Mol Cell Biol 4, 373-385.

Hao, M., Mukherjee, S., and Maxfield, F.R. (2001). Cholesterol depletion induces large scale domain segregation in living cell membranes. Proc Natl Acad Sci USA 98, 13072-13077.

Harder, T., and Simons, K. (1997). Caveolae, DIGs, and the dynamics of sphingolipid-cholesterol microdomains. Curr Opin Cell Biol 9, 534-542.

Harder, T., Scheiffele, P., Verkade, P., and Simons, K. (1998). Lipid domain structure of the plasma membrane revealed by patching of membrane components. J Cell Biol 141, 929-942.

Haynes, M.P., Phillips, M.C., and Rothblat, G.H. (2000). Efflux of cholesterol from different cellular pools. Biochemistry 39, 4508-4517.

Heintzmann, R., and Cremer, C.G. (1999). Laterally modulated excitation microscopy: improvement of resolution by using a diffraction grating. G.B. Altshuler, D.A. Benaron, B. Ehrenberg, M. Dal Fante, S.G. Bown, I.J. Bigio, G.F. Bottiroli, F. Baldini, N.I. Croitoru, T.I. Karu, et al., eds. (SPIE), pp. 185-196.

Hell, S.W. (2007). Far-Field Optical Nanoscopy. Science 316, 1153-1158.

Hell, S.W., and Wichmann, J. (1994). Breaking the diffraction resolution limit by stimulated emission: stimulated-emission-depletion fluorescence microscopy. Opt Lett 19, 780-782.

Hell, S.W. (2003). Toward fluorescence nanoscopy. Nat Biotechnol 21, 1347-1355.

Hess, S.T., Girirajan, T.P.K., and Mason, M.D. (2006). Ultra-high resolution imaging by fluorescence photoactivation localization microscopy. Biophys J 91, 4258-4272.

Hess, S.T., Gould, T.J., Gudheti, M.V., Maas, S.A., Mills, K.D., and Zimmerberg, J. (2007). Dynamic clustered distribution of hemagglutinin resolved at $40 \mathrm{~nm}$ in living cell membranes discriminates between raft theories. Proc Natl Acad Sci USA 104, 17370-17375.

Heumann, R., Kachel, V., and Thoenen, H. (1983). Relationship between NGF-mediated volume increase and "priming effect" in fast and slow reacting clones of PC12 pheochromocytoma cells. Role of cAMP. Exp Cell Res 145, 179-190. 
Holopainen, J.M., Metso, A.J., Mattila, J.-P., Jutila, A., and Kinnunen, P.K.J. (2004). Evidence for the Lack of a Specific Interaction between Cholesterol and Sphingomyelin. Biophys J 86, 1510-1520.

Holroyd, P. (2002). Imaging direct, dynamin-dependent recapture of fusing secretory granules on plasma membrane lawns from PC12 cells. Proc Natl Acad Sci USA 99, 16806-16811.

Honigmann, A., Veronika, M., Hell, S., and Eggeling, C. (2013a). STED microscopy detects and quantifies liquid phase separation in lipid membranes using a new far-red emitting fluorescent phosphoglycerolipid analogue. Faraday Discuss 161, 77-89.

Honigmann, A., van den Bogaart, G., Iraheta, E., Risselada, H.J., Milovanovic, D., Mueller, V., Müllar, S., Diederichsen, U., Fasshauer, D., Grubmüller, H., et al. (2013b). Phosphatidylinositol 4,5bisphosphate clusters act as molecular beacons for vesicle recruitment. Nat Struct Mol Biol 20, 679-686.

Hoopmann, P., Punge, A., Barysch, S.V., Westphal, V., Bückers, J., Opazo, F., Bethani, I., Lauterbach, M.A., Hell, S.W., and Rizzoli, S.O. (2010). Endosomal sorting of readily releasable synaptic vesicles. Proc Natl Acad Sci USA 107, 19055-19060.

Horger, K.S., Estes, D.J., Capone, R., and Mayer, M. (2009). Films of Agarose Enable Rapid Formation of Giant Liposomes in Solutions of Physiologic Ionic Strength. J Am Chem Soc 131, 1810-1819.

Huang, J., and Feigenson, G.W. (1999). A microscopic interaction model of maximum solubility of cholesterol in lipid bilayers. Biophys J 76, 2142-2157.

Huang, J. (2009). Model membrane thermodynamics and lateral distribution of cholesterol: from experimental data to Monte Carlo simulation. Meth Enzymol 455, 329-364.

Ikonen, E. (2008). Cellular cholesterol trafficking and compartmentalization. Nat Rev Mol Cell Biol 9, 125-138.

Jacobson, K., Ishihara, A., and Inman, R. (1987). Lateral diffusion of proteins in membranes. Annu Rev Physiol 49, 163-175.

Jain, M.K., and White, H.B. (1977). Long-range order in biomembranes. Adv Lipid Res 15, 1-60.

Kaiser, H.-J., Orłowski, A., Róg, T., Nyholm, T.K.M., Chai, W., Feizi, T., Lingwood, D., Vattulainen, I., and Simons, K. (2011). Lateral sorting in model membranes by cholesterol-mediated hydrophobic matching. Proc Natl Acad Sci USA 108, 16628-16633.

Kalay, Z., Fujiwara, T.K., and Kusumi, A. (2012). Confining domains lead to reaction bursts: reaction kinetics in the plasma membrane. PLoS ONE 7, e32948.

Karotki, L., Huiskonen, J.T., Stefan, C.J., Ziółkowska, N.E., Roth, R., Surma, M.A., Krogan, N.J., Emr, S.D., Heuser, J., Grünewald, K., et al. (2011). Eisosome proteins assemble into a membrane scaffold. J Cell Biol 195, 889-902. 
Kenworthy, A.K. (2008). Have we become overly reliant on lipid rafts? Talking Point on the involvement of lipid rafts in T-cell activation. EMBO Reports 9, 531-535.

Kenworthy, A.K., Nichols, B.J., Remmert, C.L., Hendrix, G.M., Kumar, M., Zimmerberg, J., and Lippincott-Schwartz, J. (2004). Dynamics of putative raft-associated proteins at the cell surface. J Cell Biol 165, 735-746.

Killian, J.A. (1998). Hydrophobic mismatch between proteins and lipids in membranes. BBA-Rev Biomembranes 1376, 401-406.

Killian, J.A., and Nyholm, T.K. (2006). Peptides in lipid bilayers: the power of simple models. Curr Opin Struct Biol 16, 473-479.

Klar, T.A., Jakobs, S., Dyba, M., Egner, A., and Hell, S.W. (2000). Fluorescence microscopy with diffraction resolution barrier broken by stimulated emission. Proc Natl Acad Sci USA 97, 82068210.

Klotzsch, E., and Schütz, G.J. (2012). A critical survey of methods to detect plasma membrane rafts. Philos T Roy Soc B 368, 20120033-20120033.

Kolmakov, K., Wurm, C.A., Hennig, R., Rapp, E., Jakobs, S., Belov, V.N., and Hell, S.W. (2012). Redemitting rhodamines with hydroxylated, sulfonated, and phosphorylated dye residues and their use in fluorescence nanoscopy. Chemistry 18, 12986-12998.

Kusumi, A., and Sako, Y. (1996). Cell surface organization by the membrane skeleton. Curr Opin Cell Biol 8, 566-574.

Kusumi, A., Nakada, C., Ritchie, K., Murase, K., Suzuki, K., Murakoshi, H., Kasai, R.S., Kondo, J., and Fujiwara, T. (2005). Paradigm shift of the plasma membrane concept from the two-dimensional continuum fluid to the partitioned fluid: high-speed single-molecule tracking of membrane molecules. Annu Rev Biophys Biomol Struct 34, 351-378.

Kusumi, A., Sako, Y., and Yamamoto, M. (1993). Confined lateral diffusion of membrane receptors as studied by single particle tracking (nanovid microscopy). Effects of calcium-induced differentiation in cultured epithelial cells. Biophys J 65, 2021-2040.

Kusumi, A., Shirai, Y.M., Koyama-Honda, I., Suzuki, K.G.N., and Fujiwara, T.K. (2010). Hierarchical organization of the plasma membrane: investigations by single-molecule tracking vs. fluorescence correlation spectroscopy. FEBS Lett 584, 1814-1823.

Kusumi, A., Suzuki, K.G.N., Kasai, R.S., Ritchie, K., and Fujiwara, T.K. (2011). Hierarchical mesoscale domain organization of the plasma membrane. Trends Biochem Sci 36, 604-615.

Kwik, J., Boyle, S., Fooksman, D., Margolis, L., Sheetz, M.P., and Edidin, M. (2003). Membrane cholesterol, lateral mobility, and the phosphatidylinositol 4,5-bisphosphate-dependent organization of cell actin. Proc Natl Acad Sci USA 100, 13964-13969.

Lagerholm, B.C., Weinreb, G.E., Jacobson, K., and Thompson, N.L. (2005). Detecting Microdomains 
in Intact Cell Membranes. Annu Rev Phys Chem 56, 309-336.

Lang, T., Bruns, D., Wenzel, D., Riedel, D., Holroyd, P., Thiele, C., and Jahn, R. (2001). SNAREs are concentrated in cholesterol-dependent clusters that define docking and fusion sites for exocytosis. Embo J 20, 2202-2213.

Lang, T. (2007). SNARE proteins and 'membrane rafts'. J Physiol 585, 693-698.

Lang, T. (2008). Imaging Ca2+-Triggered Exocytosis of Single Secretory Granules on Plasma Membrane Lawns from Neuroendocrine Cells. Methods Mol Biol 440, 51-59.

Lang, T., and Rizzoli, S.O. (2010). Membrane protein clusters at nanoscale resolution: more than pretty pictures. Physiology 25, 116-124.

Lange, A., Marsh, D., Wassmer, K.H., Meier, P., and Kothe, G. (1985). Electron spin resonance study of phospholipid membranes employing a comprehensive line-shape model. Biochemistry 24 , 4383-4392.

Lange, Y., Swaisgood, M.H., Ramos, B.V., and Steck, T.L. (1989). Plasma membranes contain half the phospholipid and $90 \%$ of the cholesterol and sphingomyelin in cultured human fibroblasts. J Biol Chem 264, 3786-3793.

Langmuir, I. (1917). The Constitution and Fundamental Properties of Solids and Liquids. II. Liquids. J Am Chem Soc 39, 1848-1906.

LaRocca, T.J., Crowley, J.T., Cusack, B.J., and Pathak, P. (2010). Cholesterol Lipids of Borrelia burgdorferi Form Lipid Rafts and Are Required for the Bactericidal Activity of a ComplementIndependent Antibody. Cell Host Microbe 8, 331-342.

LaRocca, T.J., Pathak, P., Chiantia, S., Toledo, A., Silvius, J.R., Benach, J.L., and London, E. (2013). Proving lipid rafts exist: membrane domains in the prokaryote Borrelia burgdorferi have the same properties as eukaryotic lipid rafts. PLoS Pathog 9, e1003353.

Lechene, C., Hillion, F., McMahon, G., Benson, D., Kleinfeld, A.M., Kampf, J.P., Distel, D., Luyten, Y., Bonventre, J., Hentschel, D., et al. (2006). High-resolution quantitative imaging of mammalian and bacterial cells using stable isotope mass spectrometry. J Biol 5, 20, 1-30.

Lee, A.G. (2003). Lipid-protein interactions in biological membranes: a structural perspective. BBA-Biomembranes 1612, 1-40.

Lee, A.G. (2011). Lipid-protein interactions. Biochem Soc Trans 39, 761-766.

Lenard, J., and Singer, S.J. (1966). Protein conformation in cell membrane preparations as studied by optical rotatory dispersion and circular dichroism. Proc Natl Acad Sci USA 56, 1828-1835.

Lenne, P.-F., Wawrezinieck, L., Conchonaud, F., Wurtz, O., Boned, A., Guo, X.-J., Rigneault, H., He, H.-T., and Marguet, D. (2006). Dynamic molecular confinement in the plasma membrane by microdomains and the cytoskeleton meshwork. Embo J 25, 3245-3256. 
Leydig, F. (1857). Lehrbuch der Histologie des Menschen und der Thiere (Frankfurt: Verlag von Meidinger Sohn \& Comp.).

Levental, I., Byfield, F.J., Chowdhury, P., Gai, F., Baumgart, T., and Janmey, P.A. (2009). Cholesterol-dependent phase separation in cell-derived giant plasma-membrane vesicles. Biochem J 424, 163-167.

Levental, I., Grzybek, M., and Simons, K. (2010a). Greasing their way: lipid modifications determine protein association with membrane rafts. Biochemistry 49, 6305-6316.

Levental, I., Lingwood, D., Grzybek, M., Coskun, U., and Simons, K. (2010b). Palmitoylation regulates raft affinity for the majority of integral raft proteins. Proc Natl Acad Sci USA 107, 2205022054.

Leydig, F. (1857). Lehrbuch der Histologie des Menschen und der Thiere (Frankfurt: Verlag von Meidinger Sohn \& Comp.).

Lillemeier, B.F., Mörtelmaier, M.A., Forstner, M.B., Huppa, J.B., Groves, J.T., and Davis, M.M. (2010). TCR and Lat are expressed on separate protein islands on T cell membranes and concatenate during activation. Nat Immunol 11, 90-96.

Lillemeier, B.F., Pfeiffer, J.R., Surviladze, Z., Wilson, B.S., and Davis, M.M. (2006). Plasma membrane-associated proteins are clustered into islands attached to the cytoskeleton. Proc Natl Acad Sci USA 103, 18992-18997.

Ling, G.N. (1984). Evolution of the Membrane and Bulk Phase Theories. In In Search of the Physical Basis of Life, (Boston, MA: Springer US), pp. 15-51.

Lingwood, D., and Simons, K. (2010). Lipid rafts as a membrane-organizing principle. Science 327, 46-50.

Lingwood, D., Kaiser, H.-J., Levental, I., and Simons, K. (2009). Lipid rafts as functional heterogeneity in cell membranes. Biochem Soc T 37, 955-960.

Lingwood, D., Ries, J., Schwille, P., and Simons, K. (2008). Plasma membranes are poised for activation of raft phase coalescence at physiological temperature. Proc Natl Acad Sci USA 105, 10005-10010.

Liu, A.P., and Fletcher, D.A. (2006). Actin polymerization serves as a membrane domain switch in model lipid bilayers. Biophys J 91, 4046-4070.

Lodish, H., Berk, A., Kaise, C.A., Krieger, M., Scott, M.P., Bretscher, A., Ploegh, H., and Matsudaira, P. (2008). Molecular Cell Biology (New York: W H Freeman \& Company).

Loeb, J. (1906). The Dynamics of Living Matter (New york: The Columbia University Press).

London, E., and Brown, D.A. (2000). Insolubility of lipids in triton X-100: physical origin and relationship to sphingolipid/cholesterol membrane domains (rafts). BBA-Biomembranes 1508, 
$182-195$.

London, E. (2005). How principles of domain formation in model membranes may explain ambiguities concerning lipid raft formation in cells. BBA-Mol Cell Res 1746, 203-220.

López, D., and Kolter, R. (2010). Functional microdomains in bacterial membranes. Gene Dev 24, 1893-1902.

Mañes, S., Mira, E., Gómez-Moutón, C., Lacalle, R.A., Keller, P., Labrador, J.P., and Martínez-A, C. (1999). Membrane raft microdomains mediate front-rear polarity in migrating cells. Embo J 18, 6211-6220.

Marlow, L., Cain, M., Pappolla, M.A., and Sambamurti, K. (2003). $\beta$-Secretase Processing of the Alzheimer's Amyloid Protein Precursor (APP). J Mol Neurosci 20, 233-240.

Marsh, D., and Horváth, L.I. (1998). Structure, dynamics and composition of the lipid-protein interface. Perspectives from spin-labelling. BBA-Rev Biomembranes 1376, 267-296.

Marsh, D., and Páli, T. (2004). The protein-lipid interface: perspectives from magnetic resonance and crystal structures. BBA-Biomembranes 1666, 118-141.

Maxfield, F.R. (2002). Plasma membrane microdomains. Curr Opin Cell Biol 14, 483-487.

McConnell, H.M., and Radhakrishnan, A. (2003). Condensed complexes of cholesterol and phospholipids. BBA-Biomembranes 1610, 159-173.

McLaughlin, S., and Murray, D. (2005). Plasma membrane phosphoinositide organization by protein electrostatics. Nature 438, 605-611.

McLaughlin, S., Wang, J., Gambhir, A., and Murray, D. (2002). PIP(2) and proteins: interactions, organization, and information flow. Annu Rev Biophys Biomol Struct 31, 151-175.

McMahon, H.T., and Gallop, J.L. (2005). Membrane curvature and mechanisms of dynamic cell membrane remodelling. Nature 438, 590-596.

Melkonian, K.A., Ostermeyer, A.G., Chen, J.Z., Roth, M.G., and Brown, D.A. (1999). Role of lipid modifications in targeting proteins to detergent-resistant membrane rafts. Many raft proteins are acylated, while few are prenylated. J Biol Chem 274, 3910-3917.

Mellquist, J.L., Kasturi, L., Spitalnik, S.L., and Shakin-Eshleman, S.H. (1998). The amino acid following an asn-X-Ser/Thr sequon is an important determinant of $\mathrm{N}$-linked core glycosylation efficiency. Biochemistry 37, 6833-6837.

Meyer, H. (1899). Zur Theorie der Alkoholnarkose. Archiv F. Experiment. Pathol. U. Pharmakol 42, 109-118.

Mileykovskaya, E., and Dowhan, W. (2009). Cardiolipin membrane domains in prokaryotes and eukaryotes. BBA-Biomembranes 1788, 2084-2091. 
Mizuno, H., Abe, M., Dedecker, P., Makino, A., Rocha, S., Ohno-Iwashita, Y., Hofkens, J., Kobayashi, T., and Miyawaki, A. (2011). Fluorescent probes for superresolution imaging of lipid domains on the plasma membrane. Chem Sci 2, 1548.

Mondal, M., Mesmin, B., Mukherjee, S., and Maxfield, F.R. (2009). Sterols are mainly in the cytoplasmic leaflet of the plasma membrane and the endocytic recycling compartment in $\mathrm{CHO}$ cells. Mol Biol Cell 20, 581-588.

Monsigny, M., Mayer, R., and Roche, A.C. (2000). Sugar-lectin interactions: sugar clusters, lectin multivalency and avidity. Carbohydr Lett 4, 35-52.

Montesano, R., Perrelet, A., Vassalli, P., and Orci, L. (1979). Absence of filipin-sterol complexes from large coated pits on the surface of culture cells. Proc Natl Acad Sci USA 76, 6391-6395.

Morone, N., Fujiwara, T., Murase, K., Kasai, R.S., Ike, H., Yuasa, S., Usukura, J., and Kusumi, A. (2006). Three-dimensional reconstruction of the membrane skeleton at the plasma membrane interface by electron tomography. J Cell Biol 174, 851-862.

Mouritsen, O.G., and Bloom, M. (1984). Mattress model of lipid-protein interactions in membranes. Biophys J 46, 141-153.

Mouritsen, O., and Zuckermann, M.J. (2004). What's So Special About Cholesterol? Lipids 39, 1101-1113.

Mouritsen, O.G. (2011). Model answers to lipid membrane questions. Cold Spring Harb Perspect Biol 3, a004622.

Möbius, W., Ohno-Iwashita, Y., van Donselaar, E.G., Oorschot, V.M.J., Shimada, Y., Fujimoto, T., Heijnen, H.F.G., Geuze, H.J., and Slot, J.W. (2002). Immunoelectron microscopic localization of cholesterol using biotinylated and non-cytolytic perfringolysin O. J Histochem Cytochem 50, 4355.

Mueller, V., Ringemann, C., Honigmann, A., Schwarzmann, G., Medda, R., Leutenegger, M., Polyakova, S., Belov, V.N., Hell, S.W., and Eggeling, C. (2011). STED nanoscopy reveals molecular details of cholesterol- and cytoskeleton-modulated lipid interactions in living cells. Biophys J 101, 1651-1660.

Mueller, V., Honigmann, A., Ringemann, C., Medda, R., Schwarzmann, G., and Eggeling, C. (2013). FCS in STED Microscopy: Studying the Nanoscale of Lipid Membrane Dynamics. Meth Enzymol 519, 1-38.

Mukherjee, S., and Maxfield, F.R. (2004). Membrane Domains. Annu Rev Cell Dev Biol 20, 839866.

Munro, S. (2003). Lipid rafts: elusive or illusive? Cell 115, 377-388.

Murase, K., Fujiwara, T., Umemura, Y., Suzuki, K., lino, R., Yamashita, H., Saito, M., Murakoshi, H., Ritchie, K., and Kusumi, A. (2004). Ultrafine membrane compartments for molecular diffusion as 
revealed by single molecule techniques. Biophys J 86, 4075-4093.

Murata, M., Peränen, J., Schreiner, R., Wieland, F., Kurzchalia, T.V., and Simons, K. (1995). VIP21/caveolin is a cholesterol-binding protein. Proc Natl Acad Sci USA 92, 10339-10343.

Murray, D.H., and Tamm, L.K. (2009). Clustering of Syntaxin-1A in Model Membranes Is Modulated by Phosphatidylinositol 4,5-Bisphosphate and Cholesterol. Biochemistry 48, 46174625.

Murray, D.H., and Tamm, L.K. (2011). Molecular mechanism of cholesterol- and polyphosphoinositide-mediated syntaxin clustering. Biochemistry 50, 9014-9022.

Müller, D.J., Helenius, J., Alsteens, D., and Dufrêne, Y.F. (2009). Force probing surfaces of living cells to molecular resolution. Nat Methods 5, 383-390.

Müsch, A., Xu, H., Shields, D., and Rodriguez-Boulan, E. (1996). Transport of vesicular stomatitis virus $\mathrm{G}$ protein to the cell surface is signal mediated in polarized and nonpolarized cells. J Cell Biol 133, 543-558.

Neumann-Giesen, C., Falkenbach, B., Beicht, P., Claasen, S., Lüers, G., Stuermer, C.A.O., Herzog, V., and Tikkanen, R. (2004). Membrane and raft association of reggie-1/flotillin-2: role of myristoylation, palmitoylation and oligomerization and induction of filopodia by overexpression. Biochem J 378, 509.

Niggli, V., Andréoli, C., Roy, C., and Mangeat, P. (1995). Identification of a phosphatidylinositol4,5-bisphosphate-binding domain in the $\mathrm{N}$-terminal region of ezrin. FEBS Lett 376, 172-176.

Niv, H., Gutman, O., Kloog, Y., and Henis, Y.I. (2002). Activated K-Ras and H-Ras display different interactions with saturable nonraft sites at the surface of live cells. J Cell Biol 157, 865-872.

Ohtani, Y., Irie, T., Uekama, K., Fukunaga, K., and Pitha, J. (1989). Differential effects of alpha-, beta- and gamma-cyclodextrins on human erythrocytes. Eur J Biochem 186, 17-22.

Ohvo-Rekilä, H. (2002). Cholesterol interactions with phospholipids in membranes. Prog Lipid Res 41, 66-97.

Opazo, F., Levy, M., Byrom, M., Schäfer, C., Geisler, C., Groemer, T.W., Ellington, A.D., and Rizzoli, S.O. (2012). Aptamers as potential tools for super-resolution microscopy. Nat Methods 9, 938939.

Opazo, F., Punge, A., Bückers, J., Hoopmann, P., Kastrup, L., Hell, S.W., and Rizzoli, S.O. (2010). Limited Intermixing of Synaptic Vesicle Components upon Vesicle Recycling. Traffic 11, 800-812.

Orth, M., and Bellosta, S. (2012). Cholesterol: its regulation and role in central nervous system disorders. Cholesterol 2012, 1-19.

Owen, D.M., Rentero, C., Rossy, J., Magenau, A., Williamson, D., Rodriguez, M., and Gaus, K. (2010). PALM imaging and cluster analysis of protein heterogeneity at the cell surface. J 
Biophoton 3, 446-454.

Owen, D.M., Williamson, D.J., Magenau, A., and Gaus, K. (2012). Sub-resolution lipid domains exist in the plasma membrane and regulate protein diffusion and distribution. Nat Commun 3 , 1256.

Parton, R.G., Hanzal-Bayer, M., and Hancock, J.F. (2006). Biogenesis of caveolae: a structural model for caveolin-induced domain formation. J Cell Sci 119, 787-796.

Pike, L.J. (2006). Rafts defined: a report on the Keystone Symposium on Lipid Rafts and Cell Function. J Lipid Res 47, 7, 1597-1598.

Pike, L.J. (2009). The challenge of lipid rafts. J Lipid Res 50 Suppl, S323-S328.

Plowman, S.J., Muncke, C., Parton, R.G., and Hancock, J.F. (2005). H-ras, K-ras, and inner plasma membrane raft proteins operate in nanoclusters with differential dependence on the actin cytoskeleton. Proc Natl Acad Sci USA 102, 15500-15505.

Poveda, J.A., Fernández, A.M., Encinar, J.A., and González-Ros, J.M. (2008). Protein-promoted membrane domains. BBA-Biomembranes 1778, 1583-1590.

Prior, I.A., Muncke, C., Parton, R.G., and Hancock, J.F. (2003). Direct visualization of Ras proteins in spatially distinct cell surface microdomains. J Cell Biol 160, 165-170.

Pumplin, D.W., and Bloch, R.J. (1993). The membrane skeleton. Trends Cell Biol 3, 113-117.

Quincke, G. (1888). Ueber die physikalischen Eigenschaften dünner, fester Lamellen. Ann Phys Chem 271, 561-580.

Quincke, G. (1893). Artificial Amœbæ and Protoplasm. Nature 49, 5-6.

Pockels, A. (1891). Surface Tension. Nature 43, 437-439.

Quincke, G. (1888). Ueber die physikalischen Eigenschaften dünner, fester Lamellen. Ann Phys Chem 271, 561-580.

Quincke, G. (1893). Artificial Amœbæ and Protoplasm. Nature 49, 5-6.

Radhakrishnan, A., Anderson, T.G., and McConnell, H.M. (2000). Condensed complexes, rafts, and the chemical activity of cholesterol in membranes. Proc Natl Acad Sci USA 97, 12422-12427.

Radhakrishnan, A., Li, X.M., Brown, R.E., and McConnell, H.M. (2001). Stoichiometry of cholesterol-sphingomyelin condensed complexes in monolayers. BBA-Biomemebranes 1511, 1-6.

Rayleigh, L. (1890). On the superficial viscosity of water. P R Soc London 47, 127-140.

Rajendran, L., Le Lay, S., and Illges, H. (2007). Raft association and lipid droplet targeting of flotillins are independent of caveolin. Biol Chem 388, 307-314. 
Resh, M.D. (2006). Trafficking and signaling by fatty-acylated and prenylated proteins. Nat Chem Biol 2, 584-590.

Rickman, C., Medine, C.N., Dun, A.R., Moulton, D.J., Mandula, O., Halemani, N.D., Rizzoli, S.O., Chamberlain, L.H., and Duncan, R.R. (2010). t-SNARE protein conformations patterned by the lipid microenvironment. J Biol Chem 285, 13535-13541.

Ritchie, K., and Kusumi, A. (2003). Single-particle tracking image microscopy. Meth Enzymol 360, 618-634.

Ritchie, K., lino, R., Fujiwara, T., Murase, K., and Kusumi, A. (2003). The fence and picket structure of the plasma membrane of live cells as revealed by single molecule techniques (Review). Mol Membr Biol 20, 13-18.

Robertson, J.D. (1964). Unit Membranes: A review with recent new studies of experimental alterations and a new subunit structure in synaptic membranes (New York: Academic Press Inc.).

Rothberg, K.G., Ying, Y.S., Kamen, B.A., and Anderson, R.G. (1990). Cholesterol controls the clustering of the glycophospholipid-anchored membrane receptor for 5-methyltetrahydrofolate. J Cell Biol 111, 2931-2938.

Rothberg, K.G., Heuser, J.E., Donzell, W.C., Ying, Y.-S., Glenney, J.R., and Anderson, R.G.W. (1992). Caveolin, a protein component of caveolae membrane coats. Cell 68, 673-682.

Rust, M.J., Bates, M., and Zhuang, X. (2006). Sub-diffraction-limit imaging by stochastic optical reconstruction microscopy (STORM). Nat Methods 3, 793-796.

Saito, T., and Yokosuka, T. (2006). Immunological synapse and microclusters: the site for recognition and activation of T cells. Curr Opin Immunol 18, 305-313.

Sakisaka, T., ITOH, T., Miura, K., and Takenawa, T. (1997). Phosphatidylinositol 4,5-bisphosphate phosphatase regulates the rearrangement of actin filaments. Mol Cell Biol 17, 3841-3849.

Sako, Y., and Kusumi, A. (1994). Compartmentalized structure of the plasma membrane for receptor movements as revealed by a nanometer-level motion analysis. J Cell Biol 125, 1251-1264.

Sako, Y., and Kusumi, A. (1995). Barriers for lateral diffusion of transferrin receptor in the plasma membrane as characterized by receptor dragging by laser tweezers: fence versus tether. J Cell Biol 129, 1559-1574.

Salaun, C., Gould, G.W., and Chamberlain, L.H. (2005a). Lipid raft association of SNARE proteins regulates exocytosis in PC12 cells. J Biol Chem 280, 19449-19453.

Salaun, C., Gould, G.W., and Chamberlain, L.H. (2005b). The SNARE proteins SNAP-25 and SNAP23 display different affinities for lipid rafts in PC12 cells. Regulation by distinct cysteine-rich domains. J Biol Chem 280, 1236-1240.

Sankaram, M.B., and Thompson, T.E. (1990). Interaction of cholesterol with various 
glycerophospholipids and sphingomyelin. Biochemistry 29, 10670-10675.

Schägger, H., and Jagow, von, G. (1987). Tricine-sodium dodecyl sulfate-polyacrylamide gel electrophoresis for the separation of proteins in the range from 1 to $100 \mathrm{kDa}$. Anal Biochem 166, 368-379.

Schreiber, A., Fischer, S., and Lang, T. (2012). The amyloid precursor protein forms plasmalemmal clusters via its pathogenic amyloid- $\beta$ domain. Biophys J 102, 1411-1417.

Schroeder, F., Nemecz, G., Wood, W.G., Joiner, C., Morrot, G., Ayraut-Jarrier, M., and Devaux, P.F. (1991). Transmembrane distribution of sterol in the human erythrocyte. Biochim Biophys Acta 1066, 183-192.

Schroeder, R., London, E., and Brown, D. (1994). Interactions between saturated acyl chains confer detergent resistance on lipids and glycosylphosphatidylinositol (GPI)-anchored proteins: GPI-anchored proteins in liposomes and cells show similar behavior. Proc Natl Acad Sci USA 91, 12130-12134.

Scolari, S., Engel, S., Krebs, N., Plazzo, A.P., de Almeida, R.F.M., Prieto, M., Veit, M., and Herrmann, A. (2009). Lateral distribution of the transmembrane domain of influenza virus hemagglutinin revealed by time-resolved fluorescence imaging. J Biol. Chem 284, 15708-15716.

Scott, J.L., Musselman, C.A., Adu-Gyamfi, E., Kutateladze, T.G., and Stahelin, R.V. (2012). Emerging methodologies to investigate lipid-protein interactions. Integr Biol 4, 247.

Scott, R.E. (1976). Plasma membrane vesiculation: a new technique for isolation of plasma membranes. Science 194, 743-745.

Sengupta, D. (2012). Cholesterol modulates the structure, binding modes, and energetics of caveolin-membrane interactions. J Phys Chem B 116, 14556-14564.

Sengupta, P., Jovanovic-Talisman, T., Skoko, D., Renz, M., Veatch, S.L., and Lippincott-Schwartz, J. (2011). Probing protein heterogeneity in the plasma membrane using PALM and pair correlation analysis. Nat Methods 8, 969-975.

Senyo, S.E., Steinhauser, M.L., Pizzimenti, C.L., Yang, V.K., Cai, L., Wang, M., Wu, T.-D., GuerquinKern, J.-L., Lechene, C.P., and Lee, R.T. (2013). Mammalian heart renewal by pre-existing cardiomyocytes. Nature 493, 433-436.

Sezgin, E., and Schwille, P. (2012). Model membrane platforms to study protein-membrane interactions. Mol Membr Biol 29, 144-154.

Sezgin, E., Kaiser, H.-J., Baumgart, T., Schwille, P., Simons, K., and Levental, I. (2012a). Elucidating membrane structure and protein behavior using giant plasma membrane vesicles. Nat Protoc 7 , 1042-1051.

Sezgin, E., Levental, I., Grzybek, M., Schwarzmann, G., Mueller, V., Honigmann, A., Belov, V.N., Eggeling, C., Coskun, U., Simons, K., et al. (2012b). Partitioning, diffusion, and ligand binding of 
raft lipid analogs in model and cellular plasma membranes. BBA-Biomembranes 1818, 1777-1784.

Sharpe, H.J., Stevens, T.J., and Munro, S. (2010). A Comprehensive Comparison of Transmembrane Domains Reveals Organelle-Specific Properties. Cell 142, 158-169.

Shaw, A.S. (2006). Lipid rafts: now you see them, now you don't. Nat Immunol 7, 1139-1142.

Sheetz, M.P., Schindler, M., and Koppel, D.E. (1980). Lateral mobility of integral membrane proteins is increased in spherocytic erythrocytes. Nature $285,510-511$.

Shvartsman, D.E., Gutman, O., Tietz, A., and Henis, Y.I. (2006). Cyclodextrins but not Compactin Inhibit the Lateral Diffusion of Membrane Proteins Independent of Cholesterol. Traffic 7, 917-926.

Sieber, J.J., Willig, K.I., Kutzner, C., Gerding-Reimers, C., Harke, B., Donnert, G., Rammner, B., Eggeling, C., Hell, S.W., Grubmuller, H., et al. (2007). Anatomy and Dynamics of a Supramolecular Membrane Protein Cluster. Science 317, 1072-1076.

Sieber, J.J., Willig, K.I., Heintzmann, R., Hell, S.W., and Lang, T. (2006). The SNARE motif is essential for the formation of syntaxin clusters in the plasma membrane. Biophys J 90, 2843-2851.

Silvius, J.R., del Giudice, D., and Lafleur, M. (1996). Cholesterol at different bilayer concentrations can promote or antagonize lateral segregation of phospholipids of differing acyl chain length. Biochemistry 35, 15198-15208.

Simionescu, N., Lupu, F., and Simionescu, M. (1983). Rings of membrane sterols surround the openings of vesicles and fenestrae, in capillary endothelium. J Cell Biol 97, 1592-1600.

Simons, K., and Fuller, S.D. (1985). Cell surface polarity in epithelia. Annu Rev Cell Biol 1, 243-288.

Simons, K., and Gerl, M.J. (2010). Revitalizing membrane rafts: new tools and insights. Nat Rev Mol Cell Biol 11, 688-699.

Simons, K., and Ikonen, E. (1997). Functional rafts in cell membranes. Nature 387, 569-572.

Simons, K., and Sampaio, J.L. (2011). Membrane organization and lipid rafts. Cold Spring Harb Perspect Biol 3, a004697-a004697.

Simons, K., and Toomre, D. (2000). Lipid rafts and signal transduction. Nat Rev Mol Cell Biol 1, 3139.

Simons, K., and van Meer, G. (1988). Lipid sorting in epithelial cells. Biochemistry 27, 6197-6202.

Simons, K., and Wandinger-Ness, A. (1990). Polarized sorting in epithelia. Cell 62, 207-210.

Simons, M., Keller, P., Dichgans, J., and Schulz, J.B. (2001). Cholesterol and Alzheimer's disease: is there a link? Neurology 57, 1089-1093.

Singer, S.J., and Nicolson, G.L. (1972). The fluid mosaic model of the structure of cell membranes. Science $175,720-731$. 
Singer, S.J. (1974). The molecular organization of membranes. Annu Rev Biochem 43, 805-833.

Singer, S.J. (2004). Some Early History of Membrane Molecular Biology. Annu Rev Physiol 66, 1-27.

Skibbens, J.E., Roth, M.G., and Matlin, K.S. (1989). Differential extractability of influenza virus hemagglutinin during intracellular transport in polarized epithelial cells and nonpolar fibroblasts. J Cell Biol 108, 821-832.

Smaby, J.M., Momsen, M., Kulkarni, V.S., and Brown, R.E. (1996). Cholesterol-induced interfacial area condensations of galactosylceramides and sphingomyelins with identical acyl chains. Biochemistry 35, 5696-5704.

Smaby, J.M., Brockman, H.L., and Brown, R.E. (1994). Cholesterol's Interfacial Interactions with Sphingomyelins and-Phosphatidylcholines: Hydrocarbon Chain Structure Determines the Magnitude of Condensation. Biochemistry 33, 9135-9142.

Solomon, S., Masilamani, M., Rajendran, L., Bastmeyer, M., Stuermer, C.A.O., and Illges, H. (2002). The lipid raft microdomain-associated protein reggie-1/flotillin-2 is expressed in human B cells and localized at the plasma membrane and centrosome in PBMCs. Immunobiology 205, 108-119.

Somerharju, P., Virtanen, J.A., and Cheng, K.H. (1999). Lateral organisation of membrane lipids. The superlattice view. BBA-Mol Cell Biol L 1440, 32-48.

Spira, F., Mueller, N.S., Beck, G., Olshausen, von, P., Beig, J., and Wedlich-Soldner, R. (2012). Patchwork organization of the yeast plasma membrane into numerous coexisting domains. Na. Cell Biol 14, 640-648.

Sprong, H., van der Sluijs, P., and van Meer, G. (2001). How proteins move lipids and lipids move proteins. Nat Rev Mol Cell Biol 2, 504-513.

Stahelin, R.V. (2009). Lipid binding domains: more than simple lipid effectors. J Lipid Res 50 Suppl, S299-S304.

Steck, T.L., Ye, J., and Lange, Y. (2002). Probing Red Cell Membrane Cholesterol Movement with Cyclodextrin. Biophys J 83, 2118-2125.

Steinhauser, M.L., Bailey, A.P., Senyo, S.E., Guillermier, C., Perlstein, T.S., Gould, A.P., Lee, R.T., and Lechene, C.P. (2012). Multi-isotope imaging mass spectrometry quantifies stem cell division and metabolism. Nature 481, 516-519.

Südhof, T.C. (2004). The Synaptic Vesicle Cycle. Annu Rev Neurosci 27, 509-547.

Takamori, S., Holt, M., Stenius, K., Lemke, E.A., Grønborg, M., Riedel, D., Urlaub, H., Schenck, S., Brügger, B., and Ringler, P. (2006). Molecular Anatomy of a Trafficking Organelle. Cell 127, 831846.

Tanaka, K.A.K., Suzuki, K.G.N., Shirai, Y.M., Shibutani, S.T., Miyahara, M.S.H., Tsuboi, H., Yahara, M., Yoshimura, A., Mayor, S., Fujiwara, T.K., et al. (2010). Membrane molecules mobile even after 
chemical fixation. Nat Methods 7, 865-866.

Tekaia, F., and Yeramian, E. (2006). Evolution of proteomes: fundamental signatures and global trends in amino acid compositions. BMC Genomics 7, 307.

Tekaia, F., Yeramian, E., and Dujon, B. (2002). Amino acid composition of genomes, lifestyles of organisms, and evolutionary trends: a global picture with correspondence analysis. Gene 297, 5160.

Thewalt, J.L., and Bloom, M. (1992). Phosphatidylcholine: cholesterol phase diagrams. Biophys J 63, 1176-1181.

Thompson, T.E., and Tillack, T.W. (1985). Organization of glycosphingolipids in bilayers and plasma membranes of mammalian cells. Annu Rev Biophys Biophys Chem 14, 361-386.

Thomsen, P., Roepstorff, K., Stahlhut, M., and van Deurs, B. (2002). Caveolae are highly immobile plasma membrane microdomains, which are not involved in constitutive endocytic trafficking. Mol Biol Cell 13, 238-250.

Tokuyasu, K.T. (1973). A technique for ultracryotomy of cell suspensions and tissues. J Cell Biol 57, 551-565.

Tomishige, M., and Kusumi, A. (1999). Compartmentalization of the erythrocyte membrane by the membrane skeleton: intercompartmental hop diffusion of band 3. Mol Biol Cell 10, 2475-2479.

Toulmay, A., and Prinz, W.A. (2013). Direct imaging reveals stable, micrometer-scale lipid domains that segregate proteins in live cells. J Cell Biol 202, 35-44.

Tsukita, S., and Yonemura, S. (1997). ERM (ezrin/radixin/moesin) family: from cytoskeleton to signal transduction. Curr Opin Cell Biol 9, 70-75.

Tyteca, D., D'Auria, L., Smissen, Der, P.V., Medts, T., Carpentier, S., Monbaliu, J.C., de Diesbach, P., and Courtoy, P.J. (2010). Three unrelated sphingomyelin analogs spontaneously cluster into plasma membrane micrometric domains. BBA-Biomembranes 1798, 909-927.

van den Bogaart, G., Meyenberg, K., Risselada, H.J., Amin, H., Willig, K.I., Hubrich, B.E., Dier, M., Hell, S.W., Grubmüller, H., Diederichsen, U., et al. (2011). Membrane protein sequestering by ionic protein-lipid interactions. Nature 479, 552-555.

van Donselaar, E., Posthuma, G., Zeuschner, D., Humbel, B.M., and Slot, J.W. (2007). Immunogold Labeling of Cryosections from High-Pressure Frozen Cells. Traffic 8, 471-485.

van Meer, G., Voelker, D.R., and Feigenson, G.W. (2008). Membrane lipids: where they are and how they behave. Nat Rev Mol Cell Biol 9, 112-124.

Varki, A., Cummings, R.D., Esko, J.D., Stanley, P., Bertozzi, C.R., Hart, G.W., and Etzler, M.E. (2009). Essentials of Glycobiology (New York: Cold Spring Harbor Laboratory Press). 
Veatch, S.L. (2007). From small fluctuations to large-scale phase separation: lateral organization in model membranes containing cholesterol. Semin Cell Dev Biol 18, 573-582.

Veatch, S.L., and Keller, S.L. (2005). Seeing spots: complex phase behavior in simple membranes. BBA-Mol Cell Res 1746, 172-185.

Veatch, S.L., Machta, B.B., Shelby, S.A., Chiang, E.N., Holowka, D.A., and Baird, B.A. (2012). Correlation functions quantify super-resolution images and estimate apparent clustering due to over-counting. PLoS ONE 7, e31457.

Vetrivel, K.S., Meckler, X., Chen, Y., Nguyen, P.D., Seidah, N.G., Vassar, R., Wong, P.C., Fukata, M., Kounnas, M.Z., and Thinakaran, G. (2009). Alzheimer disease Abeta production in the absence of S-palmitoylation-dependent targeting of BACE1 to lipid rafts. J Biol Chem 284, 3793-3803.

Vidugiriene, J., and Menon, A.K. (1994). The GPI anchor of cell-surface proteins is synthesized on the cytoplasmic face of the endoplasmic reticulum. J Cell Biol 127, 333.

Wallin, E., and Heijne, von, G. (1998). Genome-wide analysis of integral membrane proteins from eubacterial, archaean, and eukaryotic organisms. Protein Sci 7, 1029-1038.

Walther, T.C., Brickner, J.H., Aguilar, P.S., Bernales, S., Pantoja, C., and Walter, P. (2006). Eisosomes mark static sites of endocytosis. Nature 439, 998-1003.

Wang, J., and Richards, D.A. (2012). Segregation of PIP2 and PIP3 into distinct nanoscale regions within the plasma membrane. Biol Open 1, 857-862.

Watanabe, S., Punge, A., Hollopeter, G., Willig, K.I., Hobson, R.J., Davis, M.W., Hell, S.W., and Jorgensen, E.M. (2011). Protein localization in electron micrographs using fluorescence nanoscopy. Nat Methods 8, 80-84.

Wallach, D.F., and Zahler, P.H. (1966). Protein conformations in cellular membranes. Proc Natl Acad Sci USA 56, 1552-1559.

Wawrezinieck, L., Rigneault, H., Marguet, D., and Lenne, P.F. (2005). Fluorescence Correlation Spectroscopy Diffusion Laws to Probe the Submicron Cell Membrane Organization. Biophys J 89, 4029-4042.

Westphal, V., and Hell, S.W. (2005). Nanoscale resolution in the focal plane of an optical microscope. Phys Rev Lett 94, 143903.

Willig, K.I., Rizzoli, S.O., Westphal, V., Jahn, R., and Hell, S.W. (2006). STED microscopy reveals that synaptotagmin remains clustered after synaptic vesicle exocytosis. Nature 440, 935-939.

Wilner, S.E., Wengerter, B., Maier, K., de Lourdes Borba Magalhães, M., Del Amo, D.S., Pai, S., Opazo, F., Rizzoli, S.O., Yan, A., and Levy, M. (2012). An RNA alternative to human transferrin: a new tool for targeting human cells. Mol Ther Nucleic Acids 1, e21.

Wilson, B.S., Steinberg, S.L., Liederman, K., Pfeiffer, J.R., Surviladze, Z., Zhang, J., Samelson, L.E., 
Yang, L.-H., Kotula, P.G., and Oliver, J.M. (2004). Markers for detergent-resistant lipid rafts occupy distinct and dynamic domains in native membranes. Mol Biol Cell 15, 2580-2592.

Wood, W.G., Igbavboa, U., Müller, W.E., and Eckert, G.P. (2011). Cholesterol asymmetry in synaptic plasma membranes. J Neurochem 116, 684-689.

Wüstner, D. (2007). Plasma membrane sterol distribution resembles the surface topography of living cells. Mol Biol Cell 18, 211-228.

Yamada, E. (1955). The fine structure of the gall bladder epithelium of the mouse. J Biophys Biochem Cytol 1, 445-458.

Yechiel, E., and Edidin, M. (1987). Micrometer-scale domains in fibroblast plasma membranes. The Journal of Cell Biology 105, 755-760.

Yen, H.C.S., Xu, Q., Chou, D.M., Zhao, Z., and Elledge, S.J. (2008). Global Protein Stability Profiling in Mammalian Cells. Science 322, 918-923.

Yoshimori, T., Keller, P., Roth, M.G., and Simons, K. (1996). Different biosynthetic transport routes to the plasma membrane in BHK and CHO cells. J Cell Biol 133, 247-256.

Zeidan, Y.H., Jenkins, R.W., and Hannun, Y.A. (2008). Remodeling of cellular cytoskeleton by the acid sphingomyelinase/ceramide pathway. J Cell Biol 181, 335-350.

Zhang, D.-S., Piazza, V., Perrin, B.J., Rzadzinska, A.K., Poczatek, J.C., Wang, M., Prosser, H.M., Ervasti, J.M., Corey, D.P., and Lechene, C.P. (2012). Multi-isotope imaging mass spectrometry reveals slow protein turnover in hair-cell stereocilia. Nature 481, 520-524.

Zheng, Y.Z., Berg, K.B., and Foster, L.J. (2008). Mitochondria do not contain lipid rafts, and lipid rafts do not contain mitochondrial proteins. J Lipid Res 50, 988-998.

Zidovetzki, R. (2007). Use of cyclodextrins to manipulate plasma membrane cholesterol content. BBA-Biomembranes 1768, 1311-1324.

Zilly, F.E., Halemani, N.D., Walrafen, D., Spitta, L., Schreiber, A., Jahn, R., and Lang, T. (2011). Ca ${ }^{2+}$ induces clustering of membrane proteins in the plasma membrane via electrostatic interactions. Embo J 30, 1209-1220. 